\title{
Non-precious Metal Catalytic Systems Involving Iron or Cobalt Carboxylates and Alkyl Isocyanides for Hydrosilylation of Alkenes with Hydrosiloxanes
}

\author{
Daisuke Noda,${ }^{\dagger}$ Atsushi Tahara ${ }^{\dagger}$ Yusuke Sunada, ${ }^{\dagger}$ and Hideo Nagashima ${ }^{*}, \dagger$,
}

Institute for Materials Chemistry and Engineering and Graduate School of Engineering Sciences, Kyushu University, and CREST, Japan Science and Technology Agency (JST), Kasuga, Fukuoka 816-8580, Japan.

\section{Contents}

1. Experimental details

p. S-2

2. Actual NMR charts of all products

p. S-38 
General. Manipulation of air and moisture sensitive compounds was carried out under a dry nitrogen atmosphere using Schlenk tube techniques associated with a high-vacuum line or in the glove box which was filled with dry nitrogen. All solvents were distilled over appropriate drying reagents prior to use (THF, 1,4-dioxane, diethyl ether, hexane, pentane; $\left.\mathrm{Ph}_{2} \mathrm{CO} / \mathrm{Na}\right) .{ }^{1} \mathrm{H},{ }^{13} \mathrm{C},{ }^{19} \mathrm{~F},{ }^{29} \mathrm{Si}-\mathrm{NMR}$ spectra were recorded on a JEOL Lambda 400 or a Lambda 600 spectrometer at ambient temperature. ${ }^{1} \mathrm{H},{ }^{13} \mathrm{C},{ }^{19} \mathrm{~F},{ }^{29} \mathrm{Si}$-NMR chemical shifts ( $\delta$ values) were given in ppm relative to the solvent signal $\left({ }^{1} \mathrm{H},{ }^{13} \mathrm{C}\right)$ or standard resonances $\left({ }^{19} \mathrm{~F}\right.$ : external trifluoroacetic acid, ${ }^{29} \mathrm{Si}$ : external tetramethylsilane). Elemental analyses were performed by a Perkin Elmer 2400II/CHN analyzer. IR spectra were recorded on a JASCO FT/IR-550 spectrometer. Ethyl 4-vinylbenzoate ${ }^{1}$, 4-tolylisocyanide ${ }^{2}$, mesitylisocyanide ${ }^{2}$, diisopropylphenylisocyanide ${ }^{2}$, allyl polyethyleneglycol monomethylether ${ }^{3}$ were synthesized by the method reported in the literature. Iron and cobalt compounds were purchased from Wako Pure Chemical Industries, Ltd. and Sigma-Aldrich, respectively, and were used without further purification. Isocyanides [1-isocyanoadamantane (CNAd), n-butylisocyanide, tert-butylisocyanide, cyclohexylisocyanide, and 1,1,3,3-tetramethylbutylisocyanide] and boranes [pinacolborane (HBpin), catecholborane (HBcat), and 9-borabicyclo[3.3.1]nonane dimer (9-BBN)] were purchased from Tokyo Chemical Industries Co., Ltd. or Sigma-Aldrich, and were used without further purification. Alkenes and hydrosilanes, $\mathrm{PhMe}_{2} \mathrm{SiH}, \mathrm{Et}_{3} \mathrm{SiH},(\mathrm{EtO})_{3} \mathrm{SiH},(\mathrm{EtO})_{2} \mathrm{MeSiH},(\mathrm{MeO})_{3} \mathrm{SiH},(\mathrm{MeO})_{2} \mathrm{MeSiH}, \mathrm{Ph}_{2} \mathrm{SiH}_{2}$, and $\mathrm{PhSiH}_{3}$, were purchased from Tokyo Chemical Industries Co., Ltd. Hydrosiloxanes, 1,1,3,3,3-pentamethyldisiloxane (PMDS), 1,1,1-3-5,5,5-heptamethyltrisiloxane (MD’M), a vinylsiloxane polymer $\left[\mathrm{CH}_{2}=\mathrm{CHSiMe}_{2} \mathrm{O}\left(\mathrm{SiMe}_{2} \mathrm{O}\right)_{n} \mathrm{SiMe}_{2} \mathrm{CH}=\mathrm{CH}_{2}\right]$ and hydrosiloxane polymers $\left[\mathrm{Me}_{3} \mathrm{SiO}\left(\mathrm{SiMe}_{2} \mathrm{O}\right)_{n} \mathrm{Si}(\mathrm{H}) \mathrm{Me}_{2}\right.$ and $\left.\mathrm{Me}_{3} \mathrm{SiO}\{\mathrm{Si}(\mathrm{H}) \mathrm{MeO}\}_{\mathrm{m}} \mathrm{SiMe}_{3}\right]$ were obtained from Shin-Etsu Chemical Co. Ltd.

The yield and selectivity of the product was confirmed by both ${ }^{1} \mathrm{H}$ NMR spectrum with high signal-to-noise ratio $(\mathrm{S} / \mathrm{N})$ and $\mathrm{GC}$ analysis by using anisole as an internal standard. The yield of the product was carefully checked by GC spectrum when the yield was estimated to be $>99 \%$ in the ${ }^{1} \mathrm{H}$ NMR spectroscopy, and we confirmed that no starting material remained in each case.

Except two examples (entries 7 and 8 in Table S-5), anti-Markovnikov addition is only the reaction observed. Selectivity in this manuscript means the ratio of the desired product to the undesired alkenylsilane or internal alkenes formed by isomerization.

Preparation of iron (II) pivalate $\left[\mathrm{Fe}(\mathrm{OPv})_{2}\right]$. This compound was synthesized by a slightly modified procedure described in the literature ${ }^{4}$. Iron powder $(0.68 \mathrm{~g}, 12.2 \mathrm{mmol})$, pivalic acid $(3.55 \mathrm{~g}, 34.8 \mathrm{mmol})$ and pivalic anhydrate $(0.50 \mathrm{~mL}, 2.5 \mathrm{mmol})$ were placed in a $20 \mathrm{~mL}$ Schlenk tube. The mixture was stirred at $160{ }^{\circ} \mathrm{C}$ for $17 \mathrm{~h}$. In the course of this reaction, hydrogen gas evolution was observed, and color of the solution was changed to pale green. After the reaction, the resulting mixture was evacuated under vacuum, then dried at 100 ${ }^{\circ} \mathrm{C}$ under $8 \mathrm{~Pa}$ for $1 \mathrm{~h}$ to remove excess amount of pivalic acid and pivalic anhydrate. The crude green solid was dissolved in THF $(20 \mathrm{~mL})$, then passed through a short pad of Celite. The pale green solution was collected, and $\mathrm{Et}_{2} \mathrm{O}(40 \mathrm{~mL})$ was added. The $\mathrm{Fe}(\mathrm{OPv})_{2}$ was obtained as green crystals by recrystallization $(1.54 \mathrm{~g}, 5.97 \mathrm{mmol}$, 49\%). FT-IR (KBr, pellet) : v 2963, 2930, 2868, 1583, 1523, 1485, 1457, 1427, 1379, 1362, 1229, 1031, 938, $900,790,608,576,457 \mathrm{~cm}^{-1}$. Anal calcd for $\mathrm{C}_{10} \mathrm{H}_{18} \mathrm{O}_{4} \mathrm{Fe}$; C 46.54, $\mathrm{H}$ 7.03; found: $\mathrm{C} 46.71, \mathrm{H}$ 7.11. 
Preparation of cobalt (II) pivalate $\left[\mathrm{Co}(\mathrm{OPv})_{2}\right]$. This compound was synthesized by a slightly modified procedure described in the literature ${ }^{5,6}$. Cobalt acetate $(1.01 \mathrm{~g}, 5.7 \mathrm{mmol})$, pivalic acid $(3.31 \mathrm{~g}, 32.4 \mathrm{mmol})$ and pivalic anhydrate $(0.50 \mathrm{~mL}, 2.5 \mathrm{mmol})$ were placed in a $20 \mathrm{~mL}$ Schlenk tube, then the mixture was stirred at $160{ }^{\circ} \mathrm{C}$ for $1 \mathrm{~h}$. After the reaction, the resulting mixture was evacuated under vacuum, then dried at $100{ }^{\circ} \mathrm{C}$ under $8 \mathrm{~Pa}$ for $1 \mathrm{~h}$ to remove excess amount of pivalic acid and pivalic anhydrate. The crude purple solid was dissolved in THF $(20 \mathrm{~mL})$, then passed through a short pad of Celite. The purple solution was collected, and $\mathrm{Et}_{2} \mathrm{O}(40 \mathrm{~mL})$ was added. The $\mathrm{Co}(\mathrm{OPv})_{2}$ was obtained as purple crystals by recrystallization $(1.00 \mathrm{~g}, 3.83 \mathrm{mmol}$, 67\%). FT-IR (KBr, pellet) : v 2963, 2929, 2868, 1599, 1524, 1485, 1457, 1420, 1379, 1363, 1229, 1032, 938, $900,792,613,585,460 \mathrm{~cm}^{-1}$. Anal calcd for $\mathrm{C}_{10} \mathrm{H}_{18} \mathrm{O}_{4} \mathrm{Co}$; C 45.99, H 6.95; found: C 46.10, H 6.99.

Screening of isocyanide ligands in the iron-catalyzed hydrosilylation of styrene with PMDS using $\mathrm{Fe}(\mathrm{OPv})_{2}$ as the iron salt.

In a $6 \mathrm{~mL}$ glass vial, $\mathrm{Fe}(\mathrm{OPv})_{2}(3 \mathrm{mg}, 0.01 \mathrm{mmol})$ and an isocyanide $(0.02 \mathrm{mmol})$ were dissolved in a mixture of styrene ( $115 \mu \mathrm{L}, 1.0 \mathrm{mmol})$ and PMDS $(254 \mu \mathrm{L}, 1.3 \mathrm{mmol})$. The resulting mixture was stirred at $80{ }^{\circ} \mathrm{C}$ for 3 h. After cooling to room temperature, anisole $(108 \mathrm{mg}, 1.0 \mathrm{mmol})$ was added as an internal standard, and the yield of the product and selectivity of the reaction were determined by ${ }^{1} \mathrm{H}-\mathrm{NMR}$ spectroscopy. (Table $\mathrm{S} 1$ )

Table S1. Hydrosilylation of styrene catalyzed by $\mathrm{Fe}(\mathrm{OPv})_{2}$ using various isocyanides as the ligand.

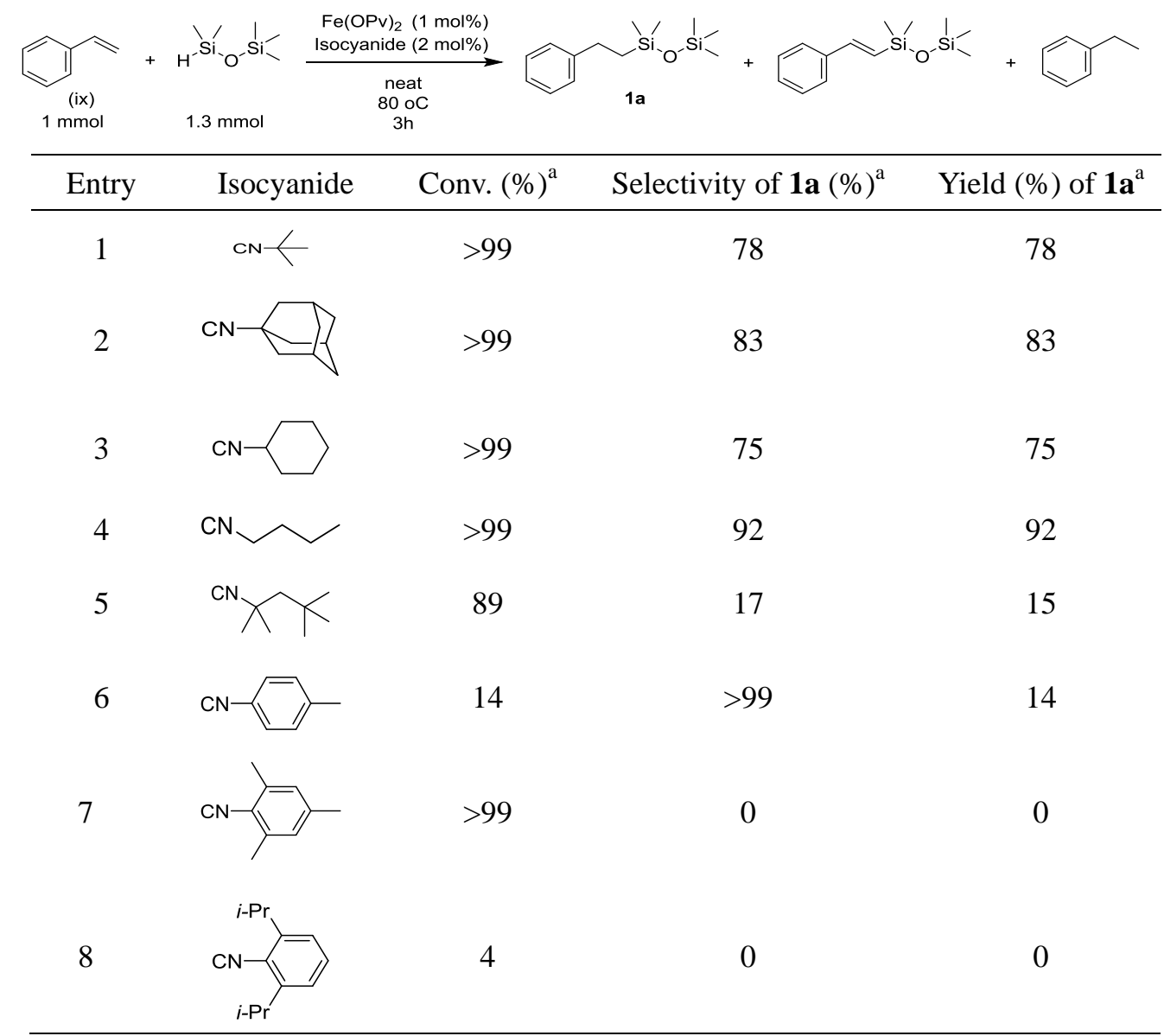

${ }^{\text {a }}$ Conversion, selectivity and the yield of the product were determined by ${ }^{1} \mathrm{H}$ NMR spectrum with anisole as an internal standard. 


\section{Screening of isocyanide ligands in the cobalt-catalyzed hydrosilylation of $\alpha$-methylstyrene with PMDS}

using $\mathrm{Co}(\mathrm{OPv})_{2}$ as the cobalt salt.

In a $6 \mathrm{~mL}$ glass vial, $\mathrm{Co}(\mathrm{OPv})_{2}(3 \mathrm{mg}, 0.01 \mathrm{mmol})$ and an isocyanide $(0.03 \mathrm{mmol})$ were dissolved in a mixture of $\alpha$-methylstyrene ( $130 \mu \mathrm{L}, 1.0 \mathrm{mmol})$ and PMDS ( $254 \mu \mathrm{L}, 1.3 \mathrm{mmol})$. The resulting mixture was stirred at 80 ${ }^{\circ} \mathrm{C}$ for $3 \mathrm{~h}$. After cooling to room temperature, anisole (108 mg, $1.0 \mathrm{mmol}$ ) was added as an internal standard, and the yield of the product and selectivity were determined by ${ }^{1} \mathrm{H}-\mathrm{NMR}$ spectroscopy. (Table S2)

Table S2. Hydrosilylation of $\alpha$-methylstyrene catalyzed by $\mathrm{Co}(\mathrm{OPv})_{2}$ using various isocyanides as the ligand.

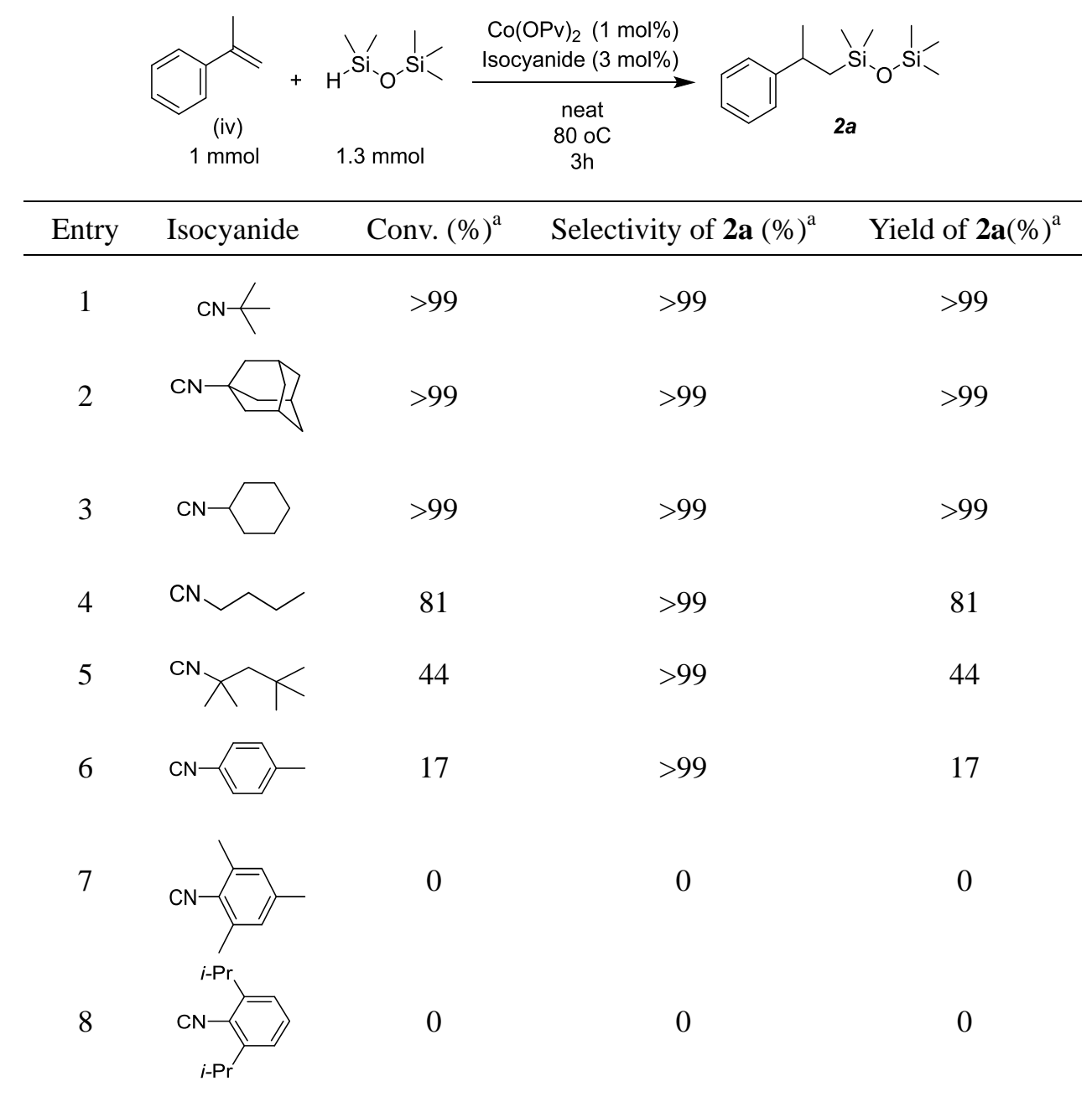

${ }^{a}$ Conversion, selectivity and the yield of the product were determined by ${ }^{1} \mathrm{H}$ NMR spectrum with anisole as an internal standard.

\section{Screening of iron salts in the iron-catalyzed hydrosilylation of styrene with PMDS using CNAd as the} ligand. (Table S3)

In a $6 \mathrm{~mL}$ glass vial were placed an iron salt [0.01 mmol (entries 9 11) or $0.03 \mathrm{mmol}$ (entries 1 8)] and CNAd ( 2 or 3 eq.), then a mixture of styrene $(115 \mu \mathrm{L}, 1.0 \mathrm{mmol})$ and PMDS $(254 \mu \mathrm{L}, 1.3 \mathrm{mmol})$ was added. The resulting mixture was stirred at $80{ }^{\circ} \mathrm{C}$ or $50{ }^{\circ} \mathrm{C}$ for $3 \mathrm{~h}$. After cooling to room temperature, anisole $(108 \mathrm{mg}, 1.0$ $\mathrm{mmol}$ ) was added as an internal standard, and the yield of the product and selectivity of the reaction were 
determined by ${ }^{1} \mathrm{H}-\mathrm{NMR}$ spectroscopy.

Table S3. Hydrosilylation of styrene catalyzed by various Fe salts using CNAd as the ligand.

\begin{tabular}{|c|c|c|c|c|c|c|}
\hline (ix) & 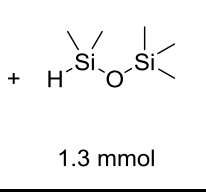 & $\begin{array}{c}\begin{array}{c}\text { Fe salt }(\times \text { mol\%) } \\
\text { CNAd }(y \text { mol\%) }\end{array} \\
\text { neat } \\
80 \mathrm{oC} \\
3 \mathrm{~h} \\
\end{array}$ & & & 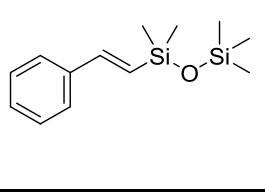 & \\
\hline Entry & Fe salt & $\begin{array}{l}\text { Cat. Loading } \\
\text { x (mol\%) }\end{array}$ & $\begin{array}{c}\text { CNAd } \\
\text { y (mol\%) }\end{array}$ & $\begin{array}{l}\text { Conv. } \\
(\%)^{\mathrm{b}}\end{array}$ & $\begin{array}{c}\text { Selectivity of } \mathbf{1 a} \\
(\%)^{\mathrm{b}}\end{array}$ & $\begin{array}{c}\text { Yield of } \mathbf{1 a} \\
(\%)^{\mathrm{b}}\end{array}$ \\
\hline 1 & $\mathrm{FeCl}_{2}$ & 3 & 6 & 0 & 0 & 0 \\
\hline 2 & $\mathrm{FeCl}_{3}$ & 3 & 6 & 0 & 0 & 0 \\
\hline 3 & $\mathrm{FeBr}_{2}$ & 3 & 6 & 0 & 0 & 0 \\
\hline 4 & $\mathrm{Fe}(\mathrm{acac})_{2}$ & 3 & 6 & 0 & 0 & 0 \\
\hline 5 & $\mathrm{Fe}(\mathrm{acac})_{3}$ & 3 & 6 & 0 & 0 & 0 \\
\hline 6 & $\mathrm{Fe}(\mathrm{OAc})_{2}$ & 3 & 6 & $>99$ & 81 & 81 \\
\hline 7 & $\mathrm{Fe}(\text { stearate })_{2}$ & 3 & 9 & $>99$ & 71 & 71 \\
\hline 8 & $\mathrm{Fe}(\mathrm{OPv})_{2}$ & 3 & 6 & $>99$ & 83 & 83 \\
\hline 9 & $\mathrm{Fe}(\mathrm{OPv})_{2}$ & 1 & 2 & $>99$ & 83 & 83 \\
\hline $10^{\mathrm{a}}$ & $\mathrm{Fe}(\mathrm{OPv})_{2}$ & 1 & 2 & $>99$ & $>99$ & $>99$ \\
\hline $11^{\mathrm{a}}$ & $\mathrm{Fe}(\mathrm{OPv})_{2}$ & 1 & 3 & 0 & 0 & 0 \\
\hline
\end{tabular}

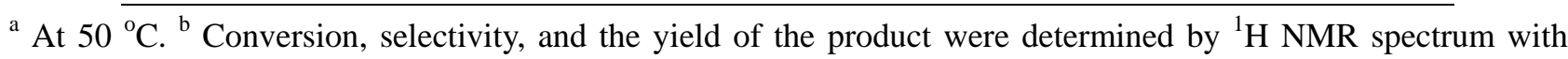
anisole as an internal standard.

\section{Screening of cobalt salts in the cobalt-catalyzed hydrosilylation of $\alpha$-methylstyrene with PMDS using} CNAd as the ligand. (Table S4)

In a $6 \mathrm{~mL}$ glass vial were placed a cobalt salt [0.01 mmol (entries 9 11) or $0.03 \mathrm{mmol}$ (entries 1 8)] and CNAd ( $2 \sim 5$ eq. to the cobalt), then a mixture of $\alpha$-methylstyrene $(130 \mu \mathrm{L}, 1.0 \mathrm{mmol})$ and PMDS $(254 \mu \mathrm{L}, 1.3 \mathrm{mmol})$ was added. The resulting mixture was stirred at $80{ }^{\circ} \mathrm{C}$ for $3 \mathrm{~h}$. After cooling to room temperature, anisole (108 $\mathrm{mg}, 1.0 \mathrm{mmol}$ ) was added as an internal standard, and the yield of the product and selectivity of the reaction were determined by ${ }^{1} \mathrm{H}-\mathrm{NMR}$ spectroscopy. 
Table S4. Hydrosilylation of $\alpha$-methylstyrene catalyzed by various Co salts using CNAd as the ligand.

\begin{tabular}{|c|c|c|c|c|c|c|}
\hline \multirow[b]{2}{*}{ Entry } & $\begin{array}{r}\text { (iv) } \\
1 \mathrm{mmc}\end{array}$ & 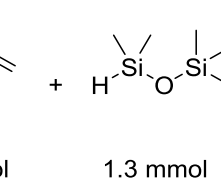 & $\begin{array}{r}\begin{array}{r}\text { Co salt } \\
\text { CNAd }\end{array} \\
\text { nea } \\
80 c \\
3 h\end{array}$ & $\begin{array}{l}\mathrm{mol} \%) \\
\mathrm{mol} \%)\end{array}$ & $2 a$ & \multirow[b]{2}{*}{$\begin{array}{c}\text { Yield of } \mathbf{2 a} \\
(\%)^{\mathrm{a}}\end{array}$} \\
\hline & Co salt & $\begin{array}{c}\text { Cat. Loading } \\
\mathrm{x}(\mathrm{mol} \%)\end{array}$ & $\begin{array}{c}\text { CNAd } \\
\mathrm{y}(\mathrm{mol} \%)\end{array}$ & $\begin{array}{l}\text { Conv. } \\
(\%)^{\mathrm{a}}\end{array}$ & $\begin{array}{c}\text { Selectivity of } \mathbf{2 a} \\
(\%)^{\mathrm{a}}\end{array}$ & \\
\hline 1 & $\mathrm{CoCl}_{2}$ & 3 & 9 & 0 & - & 0 \\
\hline 2 & $\mathrm{CoBr}_{2}$ & 3 & 9 & 0 & - & 0 \\
\hline 3 & $\mathrm{Co}(\mathrm{acac})_{2}$ & 3 & 9 & 0 & - & 0 \\
\hline 4 & $\mathrm{Co}(\mathrm{acac})_{3}$ & 3 & 9 & 0 & - & 0 \\
\hline 5 & $\mathrm{Co}(\mathrm{OAc})_{2}$ & 3 & 9 & 95 & $>99$ & 95 \\
\hline 6 & $\mathrm{Co}(\mathrm{OBz})_{2}$ & 1 & 3 & 28 & $>99$ & 28 \\
\hline 7 & $\mathrm{Co}(\mathrm{OPv})_{2}$ & 3 & 9 & $>99$ & $>99$ & $>99$ \\
\hline 8 & $\mathrm{Co}(\mathrm{OPv})_{2}$ & 1 & 3 & $>99$ & $>99$ & $>99$ \\
\hline 9 & $\mathrm{Co}(\mathrm{OPv})_{2}$ & 1 & 2 & 23 & $>99$ & 23 \\
\hline 10 & $\mathrm{Co}(\mathrm{OPv})_{2}$ & 1 & 4 & 98 & $>99$ & 98 \\
\hline 11 & $\mathrm{Co}(\mathrm{OPv})_{2}$ & 1 & 5 & 93 & $>99$ & 93 \\
\hline
\end{tabular}

${ }^{\mathrm{a}}$ Conversion, selectivity, and the yield of the product were determined by ${ }^{1} \mathrm{H}$ NMR spectrum.

To the best of our knowledge, there are no $\mathrm{Fe}(\mathrm{III})$ and $\mathrm{Co}$ (III) carboxylates. We believe that they would be catalytically active if they exist. The active species could be generated through the metathesis between the M-O bond of metal carboxylates and $\mathrm{Si}-\mathrm{H}$ bond of hydrosilanes. Because $\mathrm{Cl}$ and acac ligands strongly bound to the metal center, stronger nucleophilic activator than hydrosilanes is needed for the activation of $\mathrm{FeCl}_{3}, \mathrm{Fe}(\mathrm{acac})_{3}$ and $\mathrm{Co}(\mathrm{acac})_{3}$.

Hydrosilylation of styrene with various hydrosilanes by the $\mathrm{Fe}(\mathrm{OPv})_{2}$ / CNAd catalyst. (Table S5)

In a $6 \mathrm{~mL}$ glass vial were placed $\mathrm{Fe}(\mathrm{OPv})_{2}(8 \mathrm{mg} 0.03 \mathrm{mmol})$ and $\mathrm{CNAd}(10 \mathrm{mg}, 0.06 \mathrm{mmol})$, then a mixture of styrene $(115 \mu \mathrm{L}, 1.0 \mathrm{mmol})$ and a hydrosilane $(1.3 \mathrm{mmol})$ was added. The resulting mixture was stirred at $80{ }^{\circ} \mathrm{C}$ for $3 \mathrm{~h}$. After cooling to room temperature, anisole $(108 \mathrm{mg}, 1.0 \mathrm{mmol})$ was added as an internal standard, and the yield of the products was determined by ${ }^{1} \mathrm{H}-\mathrm{NMR}$ spectroscopy. 
Table S5. Hydrosilylation of styrene with various hydrosilanes by the $\mathrm{Fe}(\mathrm{OPv})_{2}$ / CNAd catalyst.

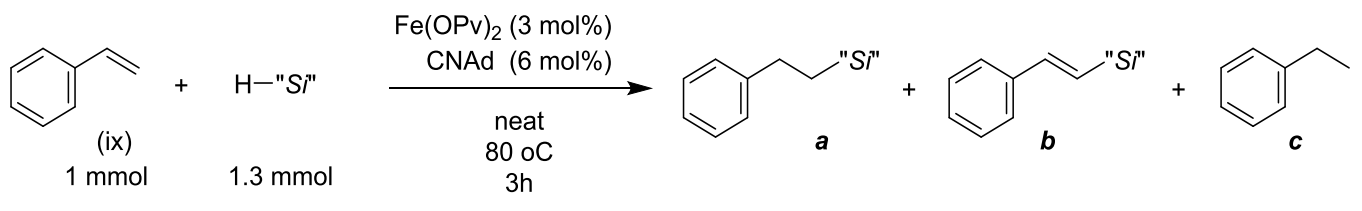

\begin{tabular}{|c|c|c|c|c|c|}
\hline \multirow{2}{*}{ Entry } & \multirow{2}{*}{ Hydrosilane } & \multirow{2}{*}{$\begin{array}{c}\text { Conv. } \\
(\%)\end{array}$} & \multicolumn{3}{|c|}{ Yield $(\%)^{\mathrm{a}}$} \\
\hline & & & $\mathbf{a}$ & b & c \\
\hline 1 & $\mathrm{PhMe}_{2} \mathrm{SiH}$ & $>99$ & 99 & 0 & 0 \\
\hline 2 & PMDS & $>99$ & 83 & 7 & 10 \\
\hline 3 & MD'M & 0 & 0 & 0 & 0 \\
\hline 4 & $\mathrm{Et}_{3} \mathrm{SiH}$ & 20 & 0 & 11 & 9 \\
\hline 5 & $(\mathrm{EtO})_{3} \mathrm{SiH}$ & 0 & 0 & 0 & 0 \\
\hline 6 & $(\mathrm{EtO})_{2} \mathrm{MeSiH}$ & 16 & 16 & 0 & 0 \\
\hline $7^{\mathrm{b}}$ & $\mathrm{PhSiH}_{3}$ & 19 & 8 & 0 & 2 \\
\hline $8^{\mathrm{c}}$ & $\mathrm{Ph}_{2} \mathrm{SiH}_{2}$ & 75 & 51 & 0 & 2 \\
\hline
\end{tabular}

${ }^{a}$ Yield of the product were determined by both GC and ${ }^{1} \mathrm{H}$ NMR spectra. ${ }^{\mathrm{b}}$ The Markovnikov product with $\mathrm{PhSiH}_{3}$ was obtained in $3 \%$ yield. ${ }^{\mathrm{c}}$ The Markovnikov product with $\mathrm{Ph}_{2} \mathrm{SiH}_{2}$ was obtained in $14 \%$ yield.

\section{Hydrosilylation of $\alpha$-methylstyrene with various hydrosilanes catalyzed by the $\operatorname{Co}(\mathrm{OPv})_{2} / \mathrm{CNAd}$ catalyst} (Table S6)

In a $6 \mathrm{~mL}$ glass vial were placed $\mathrm{Co}(\mathrm{OPv})_{2}(8 \mathrm{mg} 0.03 \mathrm{mmol})$ and CNAd $(15 \mathrm{mg}, 0.09 \mathrm{mmol})$, then a mixture of $\alpha$-methylstyrene $(130 \mu \mathrm{L}, 1.0 \mathrm{mmol})$ and a hydrosilane $(1.3 \mathrm{mmol})$ was added. The resulting mixture was stirred at $80{ }^{\circ} \mathrm{C}$ for $3 \mathrm{~h}$. After cooling to room temperature, anisole (108 mg, $1.0 \mathrm{mmol}$ ) was added as an internal standard, and the yield of the product was determined by ${ }^{1} \mathrm{H}$-NMR spectroscopy.

Table S6. Hydrosilylation of $\alpha$-methylstyrene with various hydrosilanes by the $\mathrm{Co}(\mathrm{OPv})_{2} / \mathrm{CNAd}$ catalyst.

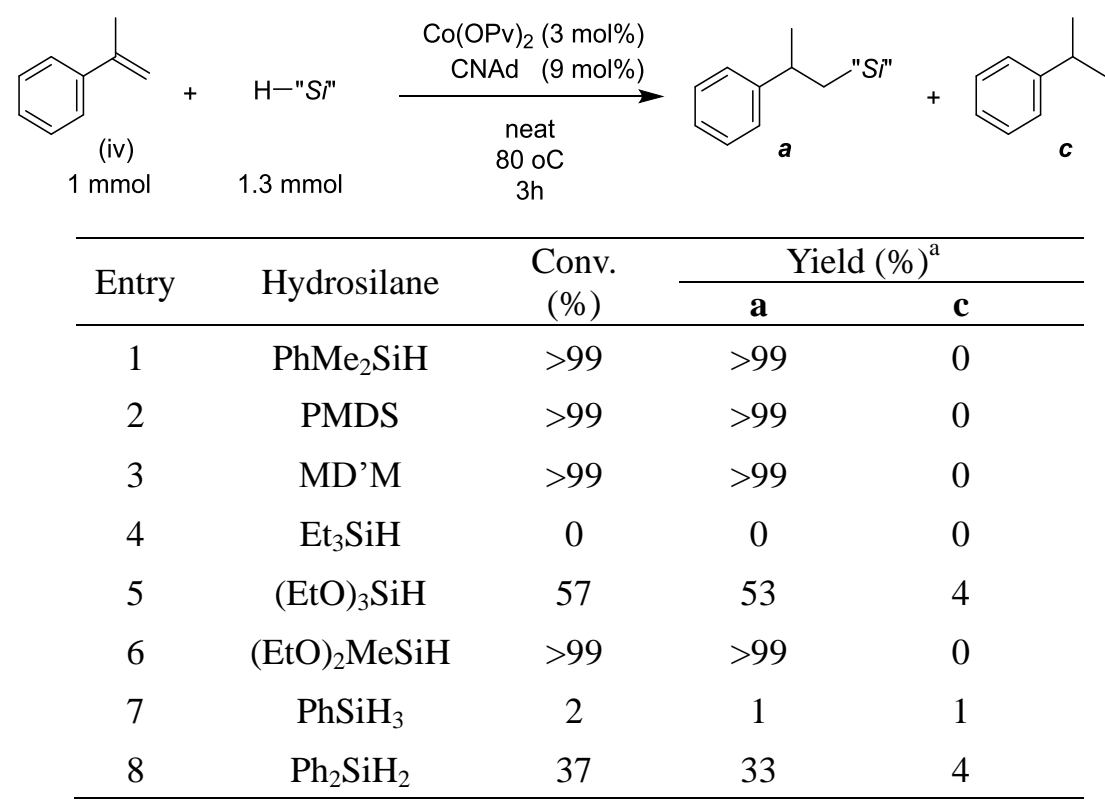

${ }^{\text {a }}$ Yield of the product were determined by both GC and ${ }^{1} \mathrm{H}$ NMR spectra. 


\section{Hydrosilylations of various alkenes with PMDS by the $\mathrm{Fe}(\mathrm{OPv})_{2}$ / CNAd catalyst. (Table S-7)}

A procedure for in entries 1 and 3-8: In a $6 \mathrm{~mL}$ glass vial, $\mathrm{Fe}(\mathrm{OPv})_{2}(3 \mathrm{mg}, 0.01 \mathrm{mmol}, 1 \mathrm{~mol} \%)$ and CNAd (3 $\mathrm{mg}, 0.02 \mathrm{mmol}, 2 \mathrm{~mol} \%)$ were dissolved in a mixture of styrene derivatives (1 mmol) and PMDS (1.3 mmol) in a $6 \mathrm{~mL}$ glass vial. The resulting mixture was stirred at the time and the temperature indicated in the table. The conversion of styrene derivatives and the yield of the product were determined by ${ }^{1} \mathrm{H}-\mathrm{NMR}$ spectroscopy using anisole as the internal standard. The products were isolated by distillation under a reduced pressure.

A procedure for entry 9 and 10: In a $6 \mathrm{~mL}$ glass vial, $\mathrm{Fe}(\mathrm{OPv})_{2}(5 \mathrm{mg}, 0.03 \mathrm{mmol})$ and CNAd $(10 \mathrm{mg}, 0.06$ mmol) were dissolved in DME $(0.10 \mathrm{~mL})$. The resulting mixture was stirred at room temperature for $1 \mathrm{~h}$, then the mixture of allyl ether $(1 \mathrm{mmol})$ and PMDS $(1.3 \mathrm{mmol})$ was added into the solution. The conversion of styrene derivatives and the yield of the product were determined by ${ }^{1} \mathrm{H}$-NMR spectroscopy using anisole as the internal standard. The products were isolated by distillation under a reduced pressure $\left(8 \mathrm{~Pa}, 65-70{ }^{\circ} \mathrm{C}\right)$.

A large scale procedure with lower catalyst loadings (entry 2$)$ : In a $200 \mathrm{~mL}$ two-neck flask, $\mathrm{Fe}(\mathrm{OPv})_{2}(5 \mathrm{mg}$, $0.02 \mathrm{mmol}, 0.01 \mathrm{~mol} \%)$ and CNAd $(26 \mathrm{mg}, 0.16 \mathrm{mmol}, 0.08 \mathrm{~mol} \%)$ were dissolved in DME $(0.1 \mathrm{~mL})$, then the mixture of styrene $(30.10 \mathrm{~g}, 200 \mathrm{mmol})$ and PMDS (21.01 g, $200 \mathrm{mmol})$ was added at room temperature. The resulting mixture was stirred at $50{ }^{\circ} \mathrm{C}$ for $24 \mathrm{~h}$. After cooling to room temperature, the conversion of styrene derivatives (97\%) and the yield (91\%) of the product were determined by ${ }^{1} \mathrm{H}-\mathrm{NMR}$ spectroscopy. The product 1a was isolated by distillation under a reduced pressure $\left(8 \mathrm{~Pa}, 65-70{ }^{\circ} \mathrm{C}\right)$. Isolated yield: $43.63 \mathrm{~g}(86 \%)$. 
Table S7. Hydrosilylation of alkenes with PMDS by the $\mathrm{Fe}(\mathrm{OPv})_{2}$ with CNAd catalyst.

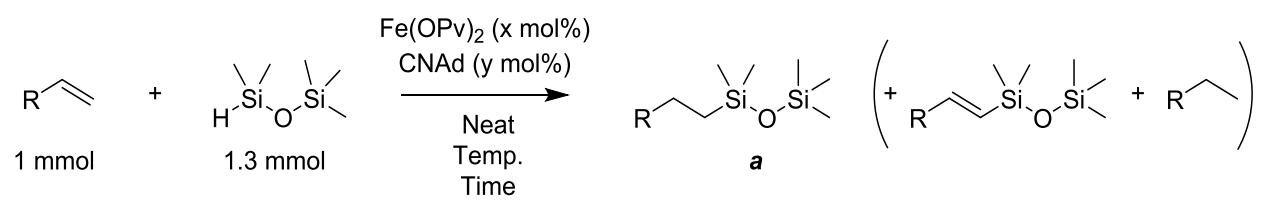

\begin{tabular}{|c|c|c|c|c|c|c|c|c|c|}
\hline Entry & Substrate & Product & $\begin{array}{c}\mathrm{Fe} \\
\text { loading } \\
(\mathrm{mol} \%)\end{array}$ & $\begin{array}{l}\text { CNAd } \\
\text { loading } \\
(\operatorname{mol} \%)\end{array}$ & $\begin{array}{l}\text { Temp. } \\
\left({ }^{\circ} \mathrm{C}\right)\end{array}$ & $\begin{array}{c}\text { Time } \\
\text { (h) }\end{array}$ & $\begin{array}{c}\text { Conv. }^{\text {a }} \\
(\%)\end{array}$ & $\begin{array}{c}\text { Selectivity }^{\mathrm{a}} \\
(\%)\end{array}$ & $\begin{array}{c}\text { Isolated } \\
\text { yield }^{\mathrm{b}}(\%)\end{array}$ \\
\hline 1 & & $1 \mathrm{a}$ & 1 & 2 & 50 & 3 & $>99$ & $>99$ & 95 \\
\hline $2^{\mathrm{c}, \mathrm{d}}$ & & $1 \mathbf{a}$ & 0.01 & 0.08 & 50 & 24 & $97^{\mathrm{e}}$ & $94^{\mathrm{e}}$ & 86 \\
\hline 3 & & 3a & 1 & 2 & 50 & 3 & $>99$ & $>99$ & 92 \\
\hline 4 & & $4 a$ & 1 & 2 & 50 & 3 & $>99$ & $>99$ & 99 \\
\hline 5 & & $5 a$ & 1 & 2 & 50 & 3 & $>99$ & $>99$ & 92 \\
\hline 6 & & $6 a$ & 1 & 2 & 50 & 3 & $>99$ & $>99$ & 92 \\
\hline 7 & & $7 a$ & 1 & 2 & 30 & 24 & $>99$ & $>99$ & 92 \\
\hline 8 & & $8 \mathbf{a}$ & 1 & 2 & 30 & 24 & $>99$ & $>99$ & 96 \\
\hline $9^{\mathrm{d}}$ & & $9 a$ & 3 & 6 & 50 & 23 & $>99$ & $92^{\mathrm{e}}$ & 88 \\
\hline $10^{\mathrm{d}}$ & & $10 \mathrm{a}$ & 3 & 6 & 50 & 23 & $>99$ & $89^{\mathrm{e}}$ & 84 \\
\hline
\end{tabular}

${ }^{\mathrm{a}}$ Conversion, selectivity, and the yield of the product were determined by ${ }^{1} \mathrm{H}$ NMR of the crude products. ${ }^{\mathrm{b}}$ The products 1a, 3a 10a were isolated by distillation. ${ }^{\mathrm{c}}$ Styrene $(200 \mathrm{mmol})$, PMDS $(200 \mathrm{mmol}){ }^{\mathrm{d}} \mathrm{DME}(0.1 \mathrm{~mL})$ was used as solvent. ${ }^{\mathrm{e}}$ Small amounts of internal alkenes were also formed.

\section{1,1,1-3,3-pentamethyl-3-phenethyldisiloxane (1a)}<smiles>C[Si](C)(C)O[Si](C)(C)CCc1ccccc1</smiles>

The product 1a was obtained from the reaction of styrene $(115 \mu \mathrm{L} 1.0 \mathrm{mmol})$ with PMDS ( $254 \mu \mathrm{L} 1.3 \mathrm{mmol})$ for $3 \mathrm{~h}$ at $50{ }^{\circ} \mathrm{C}$ catalyzed by $\mathrm{Fe}(\mathrm{OPv})_{2}(3 \mathrm{mg}, 0.01 \mathrm{mmol})$ and CNAd ( $3 \mathrm{mg}, 0.02 \mathrm{mmol}$ ) (Table $\mathrm{S} 7$, entry 1$)$. The product was isolated by distillation (8 Pa, 65-70 ${ }^{\circ} \mathrm{C}$ ). Isolated yield : $243 \mathrm{mg}(95 \%) .{ }^{1} \mathrm{H}-\mathrm{NMR}\left(395 \mathrm{MHz}, \mathrm{CDCl}_{3}\right) \delta: 0.07(\mathrm{~s}, 6 \mathrm{H}$, $-\mathrm{SiMe}_{2}$ ), 0.08 (s, 9H, - $\mathrm{SiMe}_{3}$ ), 0.86-0.92 (m, 2H, - $\left.\mathrm{CH}_{2} \mathrm{Si}\right), 2.61-2.68$ (m, 2H, - $\mathrm{CH}_{2}-$ ), 7.13-7.22 (m, 3H, Ar-H), 7.24-7.29 (m, 2H, Ar-H). ${ }^{13} \mathrm{C}-\mathrm{NMR}\left(99 \mathrm{MHz}, \mathrm{CDCl}_{3}\right) \delta: 0.3,2.0,20.4,29.4,125.5,127.8,128.3,145.2$. ${ }^{29} \mathrm{Si}-\mathrm{NMR}\left(119 \mathrm{MHz}, \mathrm{CDCl}_{3}\right) \delta: 7.09,7.52$. HRMS (EI) calcd for $\left[\mathrm{C}_{13} \mathrm{H}_{24} \mathrm{OSi}_{2}\right]: 252.1366$. found 252.1366 .

\section{1,1,1-3,3-pentamethyl-3-(2- (4-t-butylphenyl)ethyl)disiloxane (3a)}


${ }^{{ }_{\mathrm{Bu}}}$

The product 3a was obtained from the reaction of 4-t-butylstyrene (180 $\mu \mathrm{L} 1.0$ mmol) with PMDS (254 $\mu \mathrm{L} 1.3 \mathrm{mmol})$ for $3 \mathrm{~h}$ at $50{ }^{\circ} \mathrm{C}$ catalyzed by $\mathrm{Fe}(\mathrm{OPv})_{2}$

(3 mg, $0.01 \mathrm{mmol}$ ) and CNAd (3 $\mathrm{mg}, 0.02 \mathrm{mmol}$ ) (Table S7, entry 3). The product was isolated by distillation $\left(6 \mathrm{~Pa}, 70-75{ }^{\circ} \mathrm{C}\right)$. Isolated yield : $283 \mathrm{mg}(92 \%) .{ }^{1} \mathrm{H}-\mathrm{NMR}(395 \mathrm{MHz}$, $\left.\mathrm{CDCl}_{3}\right) \delta: 0.08$ (s, 9H, $\left.-\mathrm{SiMe}_{3}\right), 0.08$ (s, 6H, $-\mathrm{SiMe}_{2}$ ), 0.85-0.92 (m, 2H, - $\left.\mathrm{CH}_{2} \mathrm{Si}\right), 1.31$ (s, 9H, -CMe $)$, 2.58-2.65 (m, 2H, $\left.-\mathrm{CH}_{2}-\right), 7.14(\mathrm{~d}, J=7.7 \mathrm{~Hz}, 2 \mathrm{H}, \mathrm{Ar}-\mathrm{H}), 7.30$ (d, J=7.7 Hz, 2H, Ar-H). ${ }^{13} \mathrm{C}-\mathrm{NMR}(99 \mathrm{MHz}$, $\left.\mathrm{CDCl}_{3}\right) \delta: 0.3,2.0,20.2,28.8,31.4,34.3,125.1,127.4,142.1,148.3 .{ }^{29} \mathrm{Si}-\mathrm{NMR}\left(119 \mathrm{MHz}, \mathrm{CDCl}_{3}\right) \delta: 7.13$, 7.44. HRMS (EI) calcd for $\left[\mathrm{C}_{17} \mathrm{H}_{32} \mathrm{OSi}_{2}\right]$ : 308.1992. found 308.1991.

\section{1,1,1-3,3-pentamethyl-3-(2- (4-chlorophenyl)ethyl)disiloxane (4a)}<smiles>C[Si](C)(C)O[Si](C)(C)CCc1ccc(Cl)cc1</smiles>

The product $4 \mathbf{a}$ was obtained from the reaction of 4-chlorostyrene $(127 \mu \mathrm{L} 1.0$ mmol) with PMDS ( $254 \mu \mathrm{L} 1.3 \mathrm{mmol})$ for $3 \mathrm{~h}$ at $50{ }^{\circ} \mathrm{C}$ catalyzed by $\mathrm{Fe}(\mathrm{OPv})_{2}(3$ $\mathrm{mg}, 0.01 \mathrm{mmol}$ ) and CNAd (3 $\mathrm{mg}, 0.02 \mathrm{mmol}$ ) (Table S7, entry 4). The product was isolated by distillation $\left(8 \mathrm{~Pa}, 80-85{ }^{\circ} \mathrm{C}\right.$ ). Isolated yield : $286 \mathrm{mg}(99 \%) .{ }^{1} \mathrm{H}-\mathrm{NMR}\left(395 \mathrm{MHz}, \mathrm{CDCl}_{3}\right) \delta$ : 0.07 (s, 6H, - $\mathrm{SiMe}_{2}$ ), 0.08 (s, 9H, - $\left.\mathrm{SiMe}_{3}\right), 0.82-0.88\left(\mathrm{~m}, 2 \mathrm{H},-\mathrm{CH}_{2} \mathrm{Si}\right), 2.58-2.64\left(\mathrm{~m}, 2 \mathrm{H},-\mathrm{CH}_{2}-\right), 7.12(\mathrm{~d}$, $J=8.2 \mathrm{~Hz}, 2 \mathrm{H}, \mathrm{Ar}-\mathrm{H}), 7.23(\mathrm{~d}, J=8.2 \mathrm{~Hz}, 2 \mathrm{H}, \mathrm{Ar}-\mathrm{H}) .{ }^{13} \mathrm{C}-\mathrm{NMR}\left(99 \mathrm{MHz}, \mathrm{CDCl}_{3}\right) \delta: 0.3,2.0,20.3,28.8,128.3$, 129.2, 131.1, 143.6. ${ }^{29} \mathrm{Si}-\mathrm{NMR}\left(119 \mathrm{MHz}, \mathrm{CDCl}_{3}\right) \delta: 6.90,7.71$. HRMS (EI) calcd for $\left[\mathrm{C}_{13} \mathrm{H}_{23} \mathrm{ClOSi}_{2}\right]$ : 286.0976. found 286.0980 .

\section{1,1,1-3,3-pentamethyl-3-(2- (4-fluorophenyl)ethyl)disiloxane (5a)}

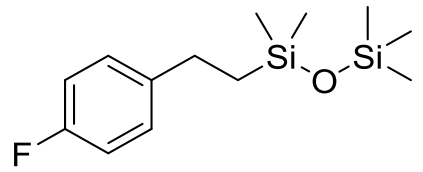

The product 5a was obtained from the reaction of 4-fluorostyrene $(154 \mu \mathrm{L} 1.0$ mmol) with PMDS $(254 \mu \mathrm{L} 1.3 \mathrm{mmol})$ for $3 \mathrm{~h}$ at $50{ }^{\circ} \mathrm{C}$ catalyzed by $\mathrm{Fe}(\mathrm{OPv})_{2}(3$ $\mathrm{mg}, 0.01 \mathrm{mmol}$ ) and CNAd (3 $\mathrm{mg}, 0.02 \mathrm{mmol}$ ) (Table S7, entry 5). The product was isolated by distillation $\left(8 \mathrm{~Pa}, 65-70{ }^{\circ} \mathrm{C}\right)$. Isolated yield : $248 \mathrm{mg}(92 \%) .{ }^{1} \mathrm{H}-\mathrm{NMR}\left(395 \mathrm{MHz}, \mathrm{CDCl}_{3}\right) \delta$ : 0.07 (s, 6H, - $\mathrm{SiMe}_{2}$ ), 0.08 (s, 9H, - $\mathrm{SiMe}_{3}$ ), 0.83-0.89 (m, 2H, - $\left.\mathrm{CH}_{2} \mathrm{Si}\right), 2.58-2.65$ (m, 2H, - $\mathrm{CH}_{2}$ ) ), 6.92-6.98 (m, $2 \mathrm{H}, \mathrm{Ar}-\mathrm{H}), 7.12-7.17$ (m, 2H, Ar-H). ${ }^{13} \mathrm{C}-\mathrm{NMR}\left(99 \mathrm{MHz}, \mathrm{CDCl}_{3}\right) \delta: 0.3,2.0,20.5,28.7,114.9$ (d, $\left.J_{C-F}=21.3\right)$, $129.1\left(\mathrm{~d}, J_{C-F}=8.2\right), 140.7\left(\mathrm{~d}, J_{C-F}=3.3\right), 161.0\left(\mathrm{~d}, J_{C-F}=242.8\right) .{ }^{19} \mathrm{~F}-\mathrm{NMR}\left(565 \mathrm{MHz}, \mathrm{CDCl}_{3}\right) \delta:-119.20$. ${ }^{29} \mathrm{Si}-\mathrm{NMR}\left(119 \mathrm{MHz}, \mathrm{CDCl}_{3}\right) \delta: 6.94,7.63$. HRMS (EI) calcd for $\left[\mathrm{C}_{13} \mathrm{H}_{23} \mathrm{FOSi}_{2}\right]: 270.1272$. found 270.1268 .

\section{Ethyl 4-(2-(1,1-3,3,3-pentamethyldisiloxanyl)ethyl)benzoate (6a)}

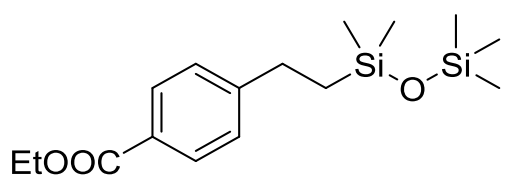

The product $\mathbf{6 a}$ was obtained from the reaction of ethyl 4-vinylbenzoate $(176 \mathrm{mg} 1.0 \mathrm{mmol})$ with PMDS $(254 \mu \mathrm{L} 1.3 \mathrm{mmol})$ for $3 \mathrm{~h}$ at $50{ }^{\circ} \mathrm{C}$ catalyzed by $\mathrm{Fe}(\mathrm{OPv})_{2}(3 \mathrm{mg}, 0.01 \mathrm{mmol})$ and CNAd $(3 \mathrm{mg}, 0.02 \mathrm{mmol})$ (Table S7, entry 6). The product was isolated by distillation (6 Pa, 135-140 ${ }^{\circ} \mathrm{C}$ ). Isolated yield : $298 \mathrm{mg}(92 \%)$. ${ }^{1} \mathrm{H}-\mathrm{NMR}\left(395 \mathrm{MHz}, \mathrm{CDCl}_{3}\right) \delta: 0.08$ (s, 6H, - $\mathrm{SiMe}_{2}$ ) $) 0.09$ (s, 9H, $\left.-\mathrm{SiMe}_{3}\right), 0.86-0.92\left(\mathrm{~m}, 2 \mathrm{H},-\mathrm{CH}_{2} \mathrm{Si}\right), 1.39(\mathrm{t}$, $\left.J=7.2 \mathrm{~Hz}, 3 \mathrm{H},-\mathrm{CH}_{3}\right), 2.66-2.72\left(\mathrm{~m}, 2 \mathrm{H},-\mathrm{CH}_{2^{-}}\right), 4.36\left(\mathrm{q}, J=7.2 \mathrm{~Hz}, 2 \mathrm{H}, \mathrm{O}-\mathrm{CH}_{2^{-}}\right), 7.26(\mathrm{~d}, J=8.2 \mathrm{~Hz}, 2 \mathrm{H}, \mathrm{Ar}-\mathrm{H})$, $7.95(\mathrm{~d}, J=8.2 \mathrm{~Hz}, 2 \mathrm{H}, \mathrm{Ar}-\mathrm{H}) .{ }^{13} \mathrm{C}-\mathrm{NMR}\left(99 \mathrm{MHz}, \mathrm{CDCl}_{3}\right) \delta: 0.3,2.0,14.3,20.1,29.6,60.7,127.8,127.9$, 129.6, 150.7, 166.7. ${ }^{29} \mathrm{Si}-\mathrm{NMR}\left(119 \mathrm{MHz}, \mathrm{CDCl}_{3}\right) \delta: 6.90,7.79$. HRMS (EI) calcd for $\left[\mathrm{C}_{16} \mathrm{H}_{28} \mathrm{O}_{3} \mathrm{Si}_{2}\right]: 324.1577$. found 324.1578 . 


\section{1,1,1-3,3-pentamethyl-3-(2- (4-anisyl)ethyl)disiloxane (7a)}

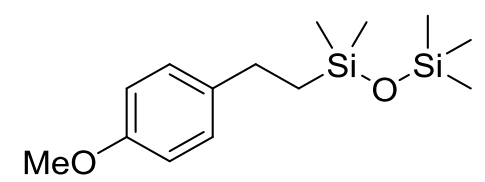

The product 7a was obtained from the reaction of 4-methoxystyrene (134 $\mu \mathrm{L}$ $1.0 \mathrm{mmol})$ with PMDS $(254 \mu \mathrm{L} 1.3 \mathrm{mmol})$ for $24 \mathrm{~h}$ at $30{ }^{\circ} \mathrm{C}$ catalyzed by $\mathrm{Fe}(\mathrm{OPv})_{2}(3 \mathrm{mg}, 0.01 \mathrm{mmol})$ and CNAd (3 $\left.\mathrm{mg}, 0.02 \mathrm{mmol}\right)$ (Table S7, entry 7). The product was isolated by distillation $\left(6 \mathrm{~Pa}, 70-75{ }^{\circ} \mathrm{C}\right)$. Isolated yield : $262 \mathrm{mg}(92 \%) .{ }^{1} \mathrm{H}-\mathrm{NMR}(395 \mathrm{MHz}$, $\left.\mathrm{CDCl}_{3}\right) \delta: 0.07$ (s, 6H, - $\mathrm{SiMe}_{2}$ ), 0.08 (s, 9H, $-\mathrm{SiMe}_{3}$ ), 0.83-0.89 (m, 2H, - $\left.\mathrm{CH}_{2} \mathrm{Si}\right), 2.56-2.62\left(\mathrm{~m}, 2 \mathrm{H},-\mathrm{CH}_{2}-\right.$ ), $3.79\left(\mathrm{~s}, 3 \mathrm{H}, \mathrm{O}-\mathrm{CH}_{3}\right), 6.82(\mathrm{~d}, J=8.7 \mathrm{~Hz}, 2 \mathrm{H}, \mathrm{Ar}-\mathrm{H}), 7.12(\mathrm{~d}, J=8.7 \mathrm{~Hz}, 2 \mathrm{H}, \mathrm{Ar}-\mathrm{H}) .{ }^{13} \mathrm{C}-\mathrm{NMR}\left(99 \mathrm{MHz}, \mathrm{CDCl}_{3}\right)$ $\delta: 0.3,2.0,20.6,28.5,55.3,113.7,128.6,137.3,157.6 .{ }^{29} \mathrm{Si}-\mathrm{NMR}\left(119 \mathrm{MHz}, \mathrm{CDCl}_{3}\right) \delta: 7.09,7.48 . \mathrm{HRMS}(\mathrm{EI})$ calcd for $\left[\mathrm{C}_{14} \mathrm{H}_{26} \mathrm{O}_{2} \mathrm{Si}_{2}\right]$ : 282.1471. found 282.1472.

\section{1,1,1-3,3-pentamethyl-3-(2- (2-tolyl)ethyl)disiloxane (8a)}<smiles>Cc1ccccc1CC[Si](C)(C)O[Si](C)(C)C</smiles>

The product 8a was obtained from the reaction of 2-methylstyrene $(129 \mu \mathrm{L} 1.0$ mmol) with PMDS $(254 \mu \mathrm{L} 1.3 \mathrm{mmol})$ for $24 \mathrm{~h}$ at $30{ }^{\circ} \mathrm{C}$ catalyzed by $\mathrm{Fe}(\mathrm{OPv})_{2}(3 \mathrm{mg}$, $0.01 \mathrm{mmol}$ ) and CNAd ( $3 \mathrm{mg}, 0.02 \mathrm{mmol}$ ) (Table S7, entry 8 ). The product was isolated by distillation $\left(6 \mathrm{~Pa}, 70-75{ }^{\circ} \mathrm{C}\right)$. Isolated yield : $255 \mathrm{mg}(96 \%) .{ }^{1} \mathrm{H}-\mathrm{NMR}\left(395 \mathrm{MHz}, \mathrm{CDCl}_{3}\right) \delta: 0.09(\mathrm{~s}$, $\left.9 \mathrm{H},-\mathrm{SiMe}_{3}\right), 0.10$ (s, 6H, $-\mathrm{SiMe}_{2}$ ) , 0.80-0.84 (m, 2H, $\left.-\mathrm{CH}_{2} \mathrm{Si}\right), 2.30\left(\mathrm{~s}, 3 \mathrm{H},-\mathrm{CH}_{3}\right), 2.58-2.62\left(\mathrm{~m}, 2 \mathrm{H},-\mathrm{CH}_{2}-\right.$ ), 7.06-7.17 (m, 4H, Ar-H). ${ }^{13} \mathrm{C}-\mathrm{NMR}\left(99 \mathrm{MHz}, \mathrm{CDCl}_{3}\right) \delta: 0.2,2.0,19.1,26.7,125.6,126.0,127.9,130.0,135.4$, 143.3. ${ }^{29} \mathrm{Si}-\mathrm{NMR}\left(119 \mathrm{MHz}, \mathrm{CDCl}_{3}\right) \delta: 7.05,7.52$. HRMS (EI) calcd for $\left[\mathrm{C}_{14} \mathrm{H}_{26} \mathrm{OSi}_{2}\right]: 266.1522$. found 266.1522 .

\section{1,1,1,3,3-pentamethyl-3-(3-(oxiran-2-ylmethoxy)propyl)disiloxane (9a)}

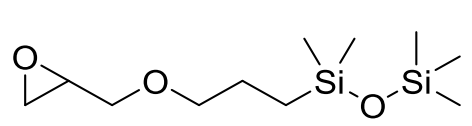

The product 9a was obtained from the reaction of allyl glycidyl ether (118 $\mu \mathrm{L}, 1 \mathrm{mmol})$ with PMDS $(254 \mu \mathrm{L}, 1.3 \mathrm{mmol})$ for $23 \mathrm{~h}$ at $50{ }^{\circ} \mathrm{C}$ catalyzed by $\mathrm{Fe}(\mathrm{OPv})_{2}(5 \mathrm{mg}, 0.03 \mathrm{mmol})$ and CNAd $(10 \mathrm{mg}, 0.06 \mathrm{mmol})$ (Table S7, entry 9).The product was isolated by distillation under a reduced pressure $\left(8 \mathrm{~Pa}, 65-70{ }^{\circ} \mathrm{C}\right)$. Isolated yield: $230 \mathrm{mg}$ $(88 \%) .{ }^{1} \mathrm{H}-\mathrm{NMR}\left(395 \mathrm{MHz}, \mathrm{CDCl}_{3}\right) \delta: 0.05$ (s, 6H, $\left.-\mathrm{SiMe}_{2}-\right), 0.06$ (s, 9H, - $\mathrm{SiMe}_{3}$ ), 0.49-0.53 (m, 2H, - $\mathrm{CH}_{2} \mathrm{Si}$ ), 1.59-1.65 (m, 2H, - $\left.\mathrm{CH}_{2^{-}}\right), 2.62(\mathrm{q}, J=2.9 \mathrm{~Hz}, 1 \mathrm{H}, \mathrm{O}-\mathrm{CH}), 2.62$ (t, $\left.J=4.4 \mathrm{~Hz}, 1 \mathrm{H}, \mathrm{O}-\mathrm{CH}\right), 3.26$ (dt, $J_{I}=2.9 \mathrm{~Hz}$, $\left.J_{2}=6.3 \mathrm{~Hz}, 1 \mathrm{H}, \mathrm{O}-\mathrm{CH}-\mathrm{C}\right), 3.37-3.51(\mathrm{~m}, 3 \mathrm{H}), 3.71\left(\mathrm{dd}, J_{l}=11.6 \mathrm{~Hz}, J_{2}=3.9 \mathrm{~Hz}, 1 \mathrm{H}\right.$, OC-CH-O). ${ }^{13} \mathrm{C}-\mathrm{NMR}$ $\left(99 \mathrm{MHz}, \mathrm{CDCl}_{3}\right) \delta: 0.2,1.9,14.2,23.4,44.2,50.8,71.4,74.3 .{ }^{29} \mathrm{Si}-\mathrm{NMR}\left(119 \mathrm{MHz}, \mathrm{CDCl}_{3}\right) \delta: 7.36,7.55$. HRMS (EI) calcd for $\left[\mathrm{C}_{11} \mathrm{H}_{26} \mathrm{O}_{3} \mathrm{Si}_{2}\right]-\mathrm{Me}: 247.1186$. found 247.1187.

\section{1-(3-(benzyloxy)propyl)-1,1,3,3,3-pentamethyldisiloxane (10a)}

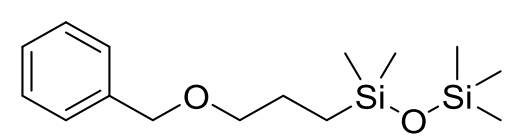

The product 10a was obtained from the reaction of allyl benzyl ether (154 $\mu \mathrm{L}, 1 \mathrm{mmol})$ with PMDS $(254 \mu \mathrm{L}, 1.3 \mathrm{mmol})$ for $23 \mathrm{~h}$ at $50{ }^{\circ} \mathrm{C}$ catalyzed by $\mathrm{Fe}(\mathrm{OPv})_{2}(5 \mathrm{mg}, 0.03 \mathrm{mmol})$ and CNAd $(10 \mathrm{mg}, 0.06 \mathrm{mmol})$ (Table S7, entry 10). The product was isolated by distillation under a reduced pressure $\left(8 \mathrm{~Pa}, 65-70{ }^{\circ} \mathrm{C}\right)$. Isolated yield: 248 $\mathrm{mg}(84 \%) .{ }^{1} \mathrm{H}-\mathrm{NMR}\left(395 \mathrm{MHz}, \mathrm{CDCl}_{3}\right) \delta: 0.05$ (s, 6H, - $\mathrm{SiMe}_{2}$ ), 0.06 (s, 9H, - $\left.\mathrm{SiMe}_{3}\right), 0.51-0.55$ (m, 2H, - $\mathrm{CH}_{2} \mathrm{Si}$ ), 1.60-1.68 (m, 2H, - $\mathrm{CH}_{2^{-}}$), 3.44 (t, J=7.3 Hz, 2H, O- $\mathrm{CH}_{2}-$ ), 4.51 (s, 2H, O- $\left.\mathrm{CH}_{2}-\mathrm{Ar}\right), 7.27-7.37$ (m, 5H, $\mathrm{Ar}-\mathrm{H}) .{ }^{13} \mathrm{C}-\mathrm{NMR}\left(99 \mathrm{MHz}, \mathrm{CDCl}_{3}\right) \delta: 0.4,2.0,14.5,23.7,72.9,73.3,127.5,127.7,128.4,138.8 .{ }^{29} \mathrm{Si}-\mathrm{NMR}$ 


\section{General Procedure for the hydrosilylation of alkenes with PMDS by the $\operatorname{Co}(\mathrm{OPv})_{2} /$ CNAd catalyst (Table} S8)

A procedure for entries 1 and 3-9: In a $6 \mathrm{~mL}$ glass vial, $\mathrm{Co}(\mathrm{OPv})_{2}(3 \mathrm{mg}, 0.01 \mathrm{mmol}$ or $26 \mathrm{mg} 0.10 \mathrm{mmol}, 1$ mol\%) and CNAd (5 mg, $0.03 \mathrm{mmol}$ or $48 \mathrm{mg} 0.30 \mathrm{mmol}, 3 \mathrm{~mol} \%$ ) were placed in a $6 \mathrm{~mL}$ glass vial, then the mixture of alkene (1 mmol or $10 \mathrm{mmol})$ and PMDS $(1.3 \mathrm{mmol}$ or $13 \mathrm{mmol})$ was added. The resulting mixture was stirred at $80^{\circ} \mathrm{C}$ for the time indicated in the table. After cooling to room temperature, the conversion of alkenes and the yield of the product were determined by ${ }^{1} \mathrm{H}-\mathrm{NMR}$ spectroscopy using anisole as the internal standard. The products were isolated by distillation under a reduced pressure or column chromatography.

A procedure for entries 10 and 11: In a $6 \mathrm{~mL}$ glass vial, $\mathrm{Co}(\mathrm{OPv})_{2}[0.01 \mathrm{mmol}$ (entry 11) or $0.03 \mathrm{mmol}$ (entry 10)] and CNAd [3 eq. to $\mathrm{Co}(\mathrm{OPv})_{2}$ ] were dissolved in DME $(0.10 \mathrm{~mL})$. The resulting mixture was stirred at room temperature for $1 \mathrm{~h}$, then the mixture of allyl ether $(1 \mathrm{mmol})$ and PMDS $(1.3 \mathrm{mmol})$ was added. The mixture was stirred at $50{ }^{\circ} \mathrm{C}$ for the time indicated in the table. The conversion of allyl ether and the yield of the product were determined by ${ }^{1} \mathrm{H}-\mathrm{NMR}$ spectroscopy using anisole as the internal standard. The products were isolated by distillation under a reduced pressure.

A large scale experiment with lower catalyst loadings (entry 2): In a $6 \mathrm{~mL}$ glass vial, $\mathrm{Co}(\mathrm{OPv})_{2}(4 \mathrm{mg}, 0.015$ mmol) and CNAd (7 mg, $0.045 \mathrm{mmol})$ were dissolved in DME (262 mg) in a $6 \mathrm{~mL}$ glass vial. Then, one-third of this solution was added to another $6 \mathrm{~mL}$ glass vial. The mixture of $\alpha$-methylstyrene $(1.18 \mathrm{~g}, 10.0 \mathrm{mmol})$ and PMDS (1.93 g, $13.0 \mathrm{mmol}$ ) was added, and the mixture was heated at $80{ }^{\circ} \mathrm{C}$ for $24 \mathrm{~h}$. After cooling to room temperature, the conversion of $\alpha$-methylstyrene (92\%) and the yield of the product $(92 \%)$ were determined by ${ }^{1} \mathrm{H}-\mathrm{NMR}$ spectroscopy using anisole as the internal standard. The product $\mathbf{2 a}$ was isolated by distillation under a reduced pressure ( $8 \mathrm{~Pa}, 60-65{ }^{\circ} \mathrm{C}$ ). Isolated yield: $2.35 \mathrm{~g}(88 \%)$. 
Table S8. Hydrosilylation of alkenes with PMDS by the $\mathrm{Co}(\mathrm{OPv})_{2} /$ CNAd catalyst.

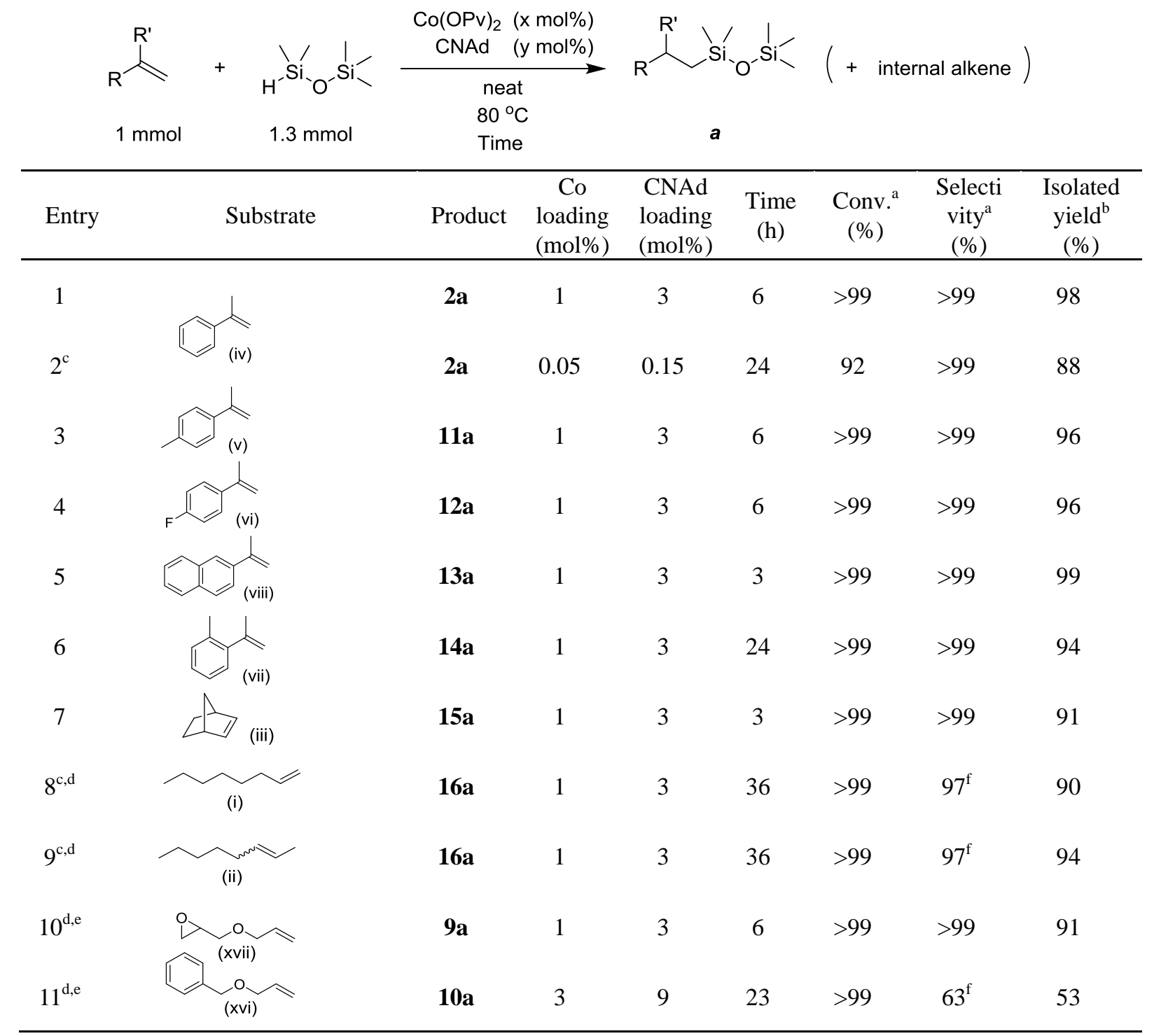

${ }^{\mathrm{a}}$ Conversion, selectivity, and the yield of the product were determined by ${ }^{1} \mathrm{H}$ NMR of the crude products. ${ }^{\mathrm{b}}$ The products 2a, 9a, 10a, 11a 16a were isolated by distillation. ${ }^{\mathrm{c}}$ alkene $(10 \mathrm{mmol})$, PMDS $(13 \mathrm{mmol}) .{ }^{\mathrm{d}} \mathrm{DME}$ was used as solvenet. ${ }^{\mathrm{f}}$ At $50{ }^{\circ} \mathrm{C} .{ }^{\mathrm{f}}$ Small amounts of internal alkenes were also formed.

\section{1,1,1-3,3-pentamethyl-3-(2-phenylpropyl)disiloxane (2a)}<smiles>CC(C[Si](C)(C)O[Si](C)(C)C)c1ccccc1</smiles>

The product $2 \mathbf{a}$ was obtained from the reaction of $\alpha$-methylstyrene $(130 \mu \mathrm{L} 1.0$ mmol) with PMDS $(254 \mu \mathrm{L} 1.3 \mathrm{mmol})$ for $6 \mathrm{~h}$ at $80{ }^{\circ} \mathrm{C}$ catalyzed by $\mathrm{Co}(\mathrm{OPv})_{2}(3 \mathrm{mg}$, $0.01 \mathrm{mmol}$ ) and CNAd (5 mg, $0.03 \mathrm{mmol}$ ) (Table S8, entry 1). The product was isolated by distillation $\left(8 \mathrm{~Pa}, 60-65{ }^{\circ} \mathrm{C}\right.$ ). Isolated yield : $260 \mathrm{mg}(98 \%) .{ }^{1} \mathrm{H}-\mathrm{NMR}\left(395 \mathrm{MHz}, \mathrm{CDCl}_{3}\right) \delta:-0.07$ (s, 3H, -SiMe-), -0.05 (s, 3H, -SiMe-), 0.05 (s, 9H, -SiMe $), 0.90-0.98$ (m, 2H, - $\mathrm{CH}_{2} \mathrm{Si}$ ), 1.28 (d, J=6.8 Hz, 3H, $-\mathrm{CH}_{3}$ ), 2.91 (sext, J=6.8 Hz, 1H, -CH-), 7.15 (t, J=6.8 Hz, 1H, Ar-H), 7.21 (d, J=6.8 Hz, 2H, Ar-H), 7.27 (t, $J=6.8 \mathrm{~Hz}, 2 \mathrm{H}, \mathrm{Ar}-\mathrm{H}) .{ }^{13} \mathrm{C}-\mathrm{NMR}\left(99 \mathrm{MHz}, \mathrm{CDCl}_{3}\right) \delta: 0.9,1.3,2.0,25.9,28.5,35.7,125.7,126.6,128.3,149.9$. ${ }^{29} \mathrm{Si}-\mathrm{NMR}\left(119 \mathrm{MHz}, \mathrm{CDCl}_{3}\right) \delta: 6.48,7.25$. HRMS (EI) calcd for $\left[\mathrm{C}_{13} \mathrm{H}_{24} \mathrm{OSi}_{2}\right]$ : 266.1522. found 266.1522. 


\section{1,1,1-3,3-pentamethyl-3-(2- (4-tolyl)propyl)disiloxane (11a)}<smiles>Cc1ccc(C(C)C[Si](C)(C)O[Si](C)(C)C)cc1</smiles>

The product 11a was obtained from the reaction of 4-isopropenyltoluene (145 $\mu \mathrm{L}$ $1.0 \mathrm{mmol}$ ) with PMDS ( $254 \mu \mathrm{L} 1.3 \mathrm{mmol})$ for $6 \mathrm{~h}$ at $80{ }^{\circ} \mathrm{C}$ catalyzed by $\mathrm{Co}(\mathrm{OPv})_{2}$ ( $3 \mathrm{mg}, 0.01 \mathrm{mmol}$ ) and CNAd (5 mg, $0.03 \mathrm{mmol}$ ) (Table S8, entry 3). The product was isolated by distillation (6 $\mathrm{Pa}, 60-65{ }^{\circ} \mathrm{C}$ ). Isolated yield : $268 \mathrm{mg}(96 \%)$. ${ }^{1} \mathrm{H}-\mathrm{NMR}(395 \mathrm{MHz}$, $\left.\mathrm{CDCl}_{3}\right) \delta:-0.06$ (s, 3H, -SiMe-), -0.04 (s, 3H, -SiMe-), 0.05 (s, 9H, - $\mathrm{SiMe}_{3}$ ), 0.88-0.98 (m, 2H, - $\mathrm{CH}_{2} \mathrm{Si}$ ), 1.26 (d, $J=6.8 \mathrm{~Hz}, 3 \mathrm{H},-\mathrm{CH}_{3}$ ), 2.31 (s, Ar-Me), 2.88 (sext, $\left.J=6.8 \mathrm{~Hz}, 1 \mathrm{H},-\mathrm{CH}-\right), 7.06-7.11$ (m, 4H, Ar-H). ${ }^{13} \mathrm{C}-\mathrm{NMR}$ $\left(99 \mathrm{MHz}, \mathrm{CDCl}_{3}\right) \delta: 0.9,1.3,2.0,21.0,26.0,28.6,35.2,126.4,128.9,135.0,147.0 .{ }^{29} \mathrm{Si}-\mathrm{NMR}(119 \mathrm{MHz}$, $\left.\mathrm{CDCl}_{3}\right) \delta: 6.51,7.17$. HRMS (EI) calcd for $\left[\mathrm{C}_{15} \mathrm{H}_{28} \mathrm{OSi}_{2}\right]: 280.1679$. found 280.1678 .

\section{1,1,1-3,3-pentamethyl-3-(2- (4-fluorophenyl)propyl)disiloxane (12a)}<smiles>CC(C[Si](C)(C)O[Si](C)(C)C)c1ccc(F)cc1</smiles>

The product 12a was obtained from the reaction of 4-fluoroisopropenylbenzene $(134 \mu \mathrm{L} 1.0 \mathrm{mmol})$ with PMDS $(254 \mu \mathrm{L} 1.3 \mathrm{mmol})$ for $6 \mathrm{~h}$ at $80{ }^{\circ} \mathrm{C}$ catalyzed by $\mathrm{Co}(\mathrm{OPv})_{2}(3 \mathrm{mg}, 0.01 \mathrm{mmol})$ and CNAd $(5 \mathrm{mg}, 0.03 \mathrm{mmol})$ (Table S8, entry 4). The product was isolated by distillation $\left(8 \mathrm{~Pa}, 60-65{ }^{\circ} \mathrm{C}\right)$. Isolated yield : $273 \mathrm{mg}(96 \%) .{ }^{1} \mathrm{H}-\mathrm{NMR}(395 \mathrm{MHz}$, $\left.\mathrm{CDCl}_{3}\right) \delta:-0.06$ (s, 6H, $-\mathrm{SiMe}_{2}$ ), 0.05 (s, 9H, $\left.-\mathrm{SiMe}_{3}\right), 0.92$ (d, J=6.8 Hz, 2H, $\left.-\mathrm{CH}_{2} \mathrm{Si}\right), 1.25$ (d, J=6.8 Hz, 3H, $-\mathrm{CH}_{3}$ ), 2.91 (sext, $\left.J=6.8 \mathrm{~Hz}, 1 \mathrm{H},-\mathrm{CH}-\right), 6.95$ (t, $\left.J=8.7 \mathrm{~Hz}, 2 \mathrm{H}, \mathrm{Ar}-\mathrm{H}\right), 7.14-7.17$ (m, 2H, Ar-H). ${ }^{13} \mathrm{C}-\mathrm{NMR}$ $\left(99 \mathrm{MHz}, \mathrm{CDCl}_{3}\right) \delta: 0.9,1.3,1.9,26.2,28.6,35.0,114.9\left(\mathrm{~d}, J_{C-F}=21.3\right), 127.9\left(\mathrm{~d}, J_{C-F}=7.4\right), 145.5\left(\mathrm{~d}, J_{C-F}=3.3\right)$, $161.1\left(\mathrm{~d}, J_{C-F}=242.8\right) .{ }^{19} \mathrm{~F}-\mathrm{NMR}\left(565 \mathrm{MHz}, \mathrm{CDCl}_{3}\right) \delta:-118.86 .{ }^{29} \mathrm{Si}-\mathrm{NMR}\left(119 \mathrm{MHz}, \mathrm{CDCl}_{3}\right) \delta: 6.32,7.44$. HRMS (EI) calcd for $\left[\mathrm{C}_{14} \mathrm{H}_{25} \mathrm{FOSi}_{2}\right]$ : 284.1428. found 284.1429.

\section{1,1,1-3,3-pentamethyl-3-(2- (2-naphthyl) propyl)disiloxane (13a)}<smiles>CC(C[Si](C)(C)O[Si](C)(C)C)c1ccc2ccccc2c1</smiles>

The product 13a was obtained from the reaction of 2-isopropenylnaphthalene (168 mg $1.0 \mathrm{mmol})$ with PMDS $(254 \mu \mathrm{L} 1.3 \mathrm{mmol})$ for $3 \mathrm{~h}$ at $80{ }^{\circ} \mathrm{C}$ catalyzed by $\mathrm{Co}(\mathrm{OPv})_{2}(3 \mathrm{mg}, 0.01 \mathrm{mmol})$ and CNAd $(5 \mathrm{mg}, 0.03 \mathrm{mmol})$ (Table $\mathrm{S} 8$, entry 5). The product was isolated by alumina column chromatography (AcOEt : hexane, 1:10 ). Isolated yield : $317 \mathrm{mg}(99 \%) .{ }^{1} \mathrm{H}-\mathrm{NMR}\left(395 \mathrm{MHz}, \mathrm{CDCl}_{3}\right) \delta:-0.05$ (s, 3H, -SiMe-), -0.04 (s, 3H, -SiMe-), 0.05 (s, 9H, $-\mathrm{SiMe}_{3}$ ), 0.98-1.10 (m, 2H, - $\mathrm{CH}_{2} \mathrm{Si}$ ), 1.36 (d, $J=6.8 \mathrm{~Hz}, 3 \mathrm{H},-\mathrm{CH}_{3}$ ), 3.10 (sext, $J=7.7 \mathrm{~Hz}, 1 \mathrm{H},-\mathrm{CH}$ ), 7.37-7.47 $(\mathrm{m}, 3 \mathrm{H}, \mathrm{Ar}-\mathrm{H}), 7.62(\mathrm{~s}, 1 \mathrm{H}, \mathrm{Ar}-\mathrm{H}), 7.76-7.80(\mathrm{~m}, 3 \mathrm{H}, \mathrm{Ar}-\mathrm{H}) .{ }^{13} \mathrm{C}-\mathrm{NMR}\left(99 \mathrm{MHz}, \mathrm{CDCl}_{3}\right) \delta: 1.0,1.4,2.0,25.8$, $28.3,35.8,124.4,124.9,125.7,125.7,127.5,127.5,127.9,132.1,133.6,147.4 .{ }^{29} \mathrm{Si}-\mathrm{NMR}\left(119 \mathrm{MHz}, \mathrm{CDCl}_{3}\right)$ $\delta: 6.51,7.36$. HRMS (EI) calcd for $\left[\mathrm{C}_{18} \mathrm{H}_{28} \mathrm{OSi}_{2}\right]: 316.1679$. found 316.1681 .

\section{1,1,1-3,3-pentamethyl-3-(2- (2-tolyl)propyl)disiloxane (14a)}<smiles>Cc1ccccc1C(C)C[Si](C)(C)O[Si](C)(C)C</smiles>

The product 14a was obtained from the reaction of 2-isopropenyltoluene $(145 \mu \mathrm{L} 1.0$ mmol) with PMDS $(254 \mu \mathrm{L} 1.3 \mathrm{mmol})$ for $24 \mathrm{~h}$ at $80{ }^{\circ} \mathrm{C}$ catalyzed by $\mathrm{Co}(\mathrm{OPv})_{2}(3$ $\mathrm{mg}, 0.01 \mathrm{mmol}$ ) and CNAd ( $5 \mathrm{mg}, 0.03 \mathrm{mmol}$ ) (Table S8, entry 6). The product was isolated by distillation $\left(10 \mathrm{~Pa}, 70-75{ }^{\circ} \mathrm{C}\right.$ ). Isolated yield : $265 \mathrm{mg}(94 \%) .{ }^{1} \mathrm{H}-\mathrm{NMR}\left(395 \mathrm{MHz}, \mathrm{CDCl}_{3}\right) \delta:-0.03$ (s, 3H, -SiMe-), -0.01 (s, 3H, -SiMe-), 0.06 (s, 9H, -SiMe 3 ), 0.87-0.99 (m, 2H, - $\mathrm{CH}_{2} \mathrm{Si}$ ), 1.24 (d, J=6.8 Hz, 3H, $-\mathrm{CH}_{3}$ ), 2.33 (s, Ar-Me), 3.19 (sext, J=6.8 Hz, 1H, -CH-), 7.04-7.19 (m, 3H, Ar-H), 7.25 (d, J=7.7 Hz, Ar-H). 
${ }^{13} \mathrm{C}-\mathrm{NMR}\left(99 \mathrm{MHz}, \mathrm{CDCl}_{3}\right) \delta: 1.0,1.4,2.0,19.4,24.8,27.7,30.0,125.3,126.2,130.1,134.3,148.1 .{ }^{29} \mathrm{Si}-\mathrm{NMR}$ $\left(119 \mathrm{MHz}, \mathrm{CDCl}_{3}\right) \delta: 6.59,7.40$. HRMS (EI) calcd for $\left[\mathrm{C}_{15} \mathrm{H}_{28} \mathrm{OSi}_{2}\right]: 280.1679$. found 280.1677 .

\section{1-(bicyclo[2.2.1]heptan-2-yl)-1,1-3,3,3-pentamethyldisiloxane (15a)}

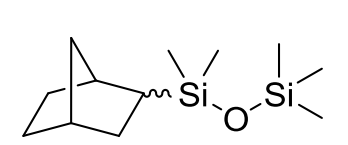

The product 15a was obtained from the reaction of 2-norbornene $(94 \mathrm{mg} 1.0 \mathrm{mmol}$ ) with PMDS $(254 \mu \mathrm{L} 1.3 \mathrm{mmol})$ for $3 \mathrm{~h}$ at $80{ }^{\circ} \mathrm{C}$ catalyzed by $\mathrm{Co}(\mathrm{OPv})_{2}(3 \mathrm{mg}, 0.01$ $\mathrm{mmol}$ ) and CNAd (5 $\mathrm{mg}, 0.03 \mathrm{mmol}$ ) (Table $\mathrm{S} 8$, entry 7). The product was isolated by distillation $\left(6 \mathrm{~Pa}, 60-65{ }^{\circ} \mathrm{C}\right)$. Isolated yield: $222 \mathrm{mg}(91 \%)$. GLC (TC-1 $(30 \mathrm{~m})$ column temperature $\left.120{ }^{\circ} \mathrm{C}\right) \mathrm{t}_{\mathrm{R}}$ $14.3 \mathrm{~min}$ (endo), $14.8 \mathrm{~min}$ (exo). exo : endo = 91.1 : 8.9. ${ }^{1} \mathrm{H}-\mathrm{NMR}\left(395 \mathrm{MHz}, \mathrm{CDCl}_{3}\right) \delta:-0.01(\mathrm{~s}, 3 \mathrm{H},-\mathrm{SiMe}$, exo), 0.00 (s, 3H, -SiMe, exo), 0.04 (s, 0.38H), 0.06 (s, 9H, -SiMe 3 , exo), 0.06 (s, 1.01H), 0.47-0.51 (m, 1H, -CHSi, exo), 0.80-0.87 (m, 0.16H), 1.06-1.10 (m, 1.26H, exo and endo), 1.18-1.23 (m, 3.71H, exo and endo), 1.32-1.36 (m, 1.25H, exo and endo), 1.37-1.49 (m, 1.24H, exo and endo), 1.51-1.54 (m, 2.39H, exo and endo), 1.59-1.69 (m, 0.19H), 2.19-2.32 (m, 2.29H, exo and endo). (Assignment of the exo- and endo- isomer was performed by ${ }^{1} \mathrm{H}_{-}{ }^{1} \mathrm{H}$ COSY spectrum (Figure S19-4) and NOE analysis (Figure S19-5)). ${ }^{13} \mathrm{C}-\mathrm{NMR}$ (99MHz, $\left.\mathrm{CDCl}_{3}\right) \delta:-0.9$ (exo), -0.7(exo), 0.6, 0.6, 2.0(exo), 27.2, 29.0(exo), 29.8, 30.3, 30.8(exo), 30.9, 31.8(exo), 34.1(exo), 36.7(exo), 37.2, 37.4(exo), 37.7(exo), 39.0, 41.7. (Assignment of the exo- and endo-isomer was performed by analyzing the intensity of each peak). ${ }^{29} \mathrm{Si}-\mathrm{NMR}\left(119 \mathrm{MHz}, \mathrm{CDCl}_{3}\right) \delta: 6.01,6.67{ }^{29} \mathrm{Si}-\mathrm{NMR}$ $\left(119 \mathrm{MHz}, \mathrm{CDCl}_{3}\right) \delta: 6.59$ (exo), 6.67, 6.78(exo), 7.13. HRMS (EI) calcd for $\left[\mathrm{C}_{12} \mathrm{H}_{26} \mathrm{OSi}_{2}\right]: 242.1522$. found 242.1523 .

\section{1,1,1-3,3-pentamethyl-3-octyldisiloxane (16a)}

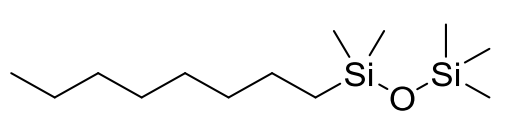

The product 16a as obtained from the reaction of 1-octene or 2-octene $(1.12 \mathrm{~g}, 10.0 \mathrm{mmol})$ with PMDS (1.93 g $13.0 \mathrm{mmol})$ for $36 \mathrm{~h}$ at $80^{\circ} \mathrm{C}$ catalyzed by $\mathrm{Co}(\mathrm{OPv})_{2}(26 \mathrm{mg}, 0.10 \mathrm{mmol})$ and CNAd $(48 \mathrm{mg}, 0.30$ mmol) (Table S8, entry 8, 9). The product was isolated by distillation $\left(8 \mathrm{~Pa}, 60{ }^{\circ} \mathrm{C}\right)$. Isolated yield : $1.167 \mathrm{~g}$ $(90 \%)$ in 1-octene, $1.222 \mathrm{~g}(94 \%)$ in 2-octene. ${ }^{1} \mathrm{H}-\mathrm{NMR}\left(395 \mathrm{MHz}, \mathrm{CDCl}_{3}\right) \delta: 0.03\left(\mathrm{~s}, 6 \mathrm{H},-\mathrm{SiMe}_{2}\right), 0.06$ (s, $9 \mathrm{H}$, $\left.-\mathrm{SiMe}_{3}\right), 0.48-0.52\left(\mathrm{~m}, 2 \mathrm{H},-\mathrm{CH}_{2} \mathrm{Si}\right), 0.88\left(\mathrm{t}, \mathrm{J}=6.8 \mathrm{~Hz}, 3 \mathrm{H},-\mathrm{CH}_{3}\right), 1.21-1.37$ (m, $\left.12 \mathrm{H},-\mathrm{CH}_{2}-\right) .{ }^{13} \mathrm{C}-\mathrm{NMR}$ $\left(99 \mathrm{MHz}, \mathrm{CDCl}_{3}\right) \delta: 0.3,2.0,14.1,18.4,22.7,23.3,29.3,29.4,31.9,33.4 .{ }^{29} \mathrm{Si}-\mathrm{NMR}\left(119 \mathrm{MHz}, \mathrm{CDCl}_{3}\right) \delta: 6.98$, 7.63. HRMS (EI) calcd for $\left[\mathrm{C}_{12} \mathrm{H}_{29} \mathrm{OSi}_{2}\right]-\mathrm{Me}: 245.1757$. found 245.1757 .

\section{1,1,1,3,3-pentamethyl-3-(3-(oxiran-2-ylmethoxy)propyl)disiloxane (9a)}

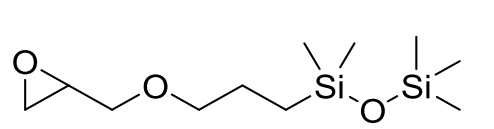

The product 9a was obtained from the reaction of allyl glycidyl ether (118 $\mu \mathrm{L}, 1 \mathrm{mmol})$ with PMDS $(254 \mu \mathrm{L}, 1.3 \mathrm{mmol})$ for $23 \mathrm{~h}$ at $50{ }^{\circ} \mathrm{C}$ catalyzed by $\mathrm{Co}(\mathrm{OPv})_{2}(3 \mathrm{mg}, 0.01 \mathrm{mmol})$ and CNAd $(5 \mathrm{mg}, 0.03 \mathrm{mmol})$ (Table S8, entry 10). The product was isolated by distillation under a reduced pressure $\left(8 \mathrm{~Pa}, 65-70{ }^{\circ} \mathrm{C}\right)$. Isolated yield : $240 \mathrm{mg}$ $(91 \%) .{ }^{1} \mathrm{H}-\mathrm{NMR}\left(395 \mathrm{MHz}, \mathrm{CDCl}_{3}\right) \delta: 0.05$ (s, 6H, - $\left.\mathrm{SiMe}_{2}-\right), 0.06$ (s, 9H, $\left.-\mathrm{SiMe}_{3}\right), 0.49-0.53\left(\mathrm{~m}, 2 \mathrm{H},-\mathrm{CH}_{2} \mathrm{Si}\right.$ ), 1.59-1.65 (m, 2H, - $\mathrm{CH}_{2^{-}}$), 2.62 (q, J=2.9 Hz, 1H, O-CH), 2.62 (t, J=4.4 Hz, 1H, O-CH), 3.26 (dt, $J_{l}=2.9 \mathrm{~Hz}$, $\left.J_{2}=6.3 \mathrm{~Hz}, 1 \mathrm{H}, \mathrm{O}-\mathrm{CH}-\mathrm{C}\right), 3.37-3.51(\mathrm{~m}, 3 \mathrm{H}), 3.71\left(\mathrm{dd}, J_{l}=11.6 \mathrm{~Hz}, J_{2}=3.9 \mathrm{~Hz}, 1 \mathrm{H}\right.$, OC-CH-O). ${ }^{13} \mathrm{C}-\mathrm{NMR}$ $\left(99 \mathrm{MHz}, \mathrm{CDCl}_{3}\right) \delta: 0.2,1.9,14.2,23.4,44.2,50.8,71.4,74.3 .{ }^{29} \mathrm{Si}-\mathrm{NMR}\left(119 \mathrm{MHz}, \mathrm{CDCl}_{3}\right) \delta: 7.36,7.55$. 
HRMS (EI) calcd for $\left[\mathrm{C}_{11} \mathrm{H}_{26} \mathrm{O}_{3} \mathrm{Si}_{2}\right]-\mathrm{Me}: 247.1186$. found 247.1187.

\section{1-(3-(benzyloxy)propyl)-1,1,3,3,3-pentamethyldisiloxane (10a)}

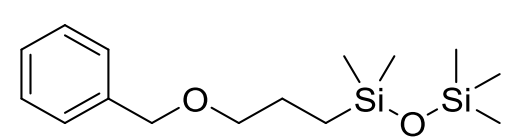

The product 10a was obtained from the reaction of allyl benzyl ether (154 $\mu \mathrm{L}, 1 \mathrm{mmol})$ with PMDS $(254 \mu \mathrm{L}, 1.3 \mathrm{mmol})$ for $23 \mathrm{~h}$ at $50{ }^{\circ} \mathrm{C}$ catalyzed by $\mathrm{Co}(\mathrm{OPv})_{2}(8 \mathrm{mg}, 0.03 \mathrm{mmol})$ and CNAd $(15 \mathrm{mg}, 0.09 \mathrm{mmol})($ Table $\mathrm{S} 8$, entry 11). The product was isolated by distillation under a reduced pressure $\left(8 \mathrm{~Pa}, 65-70{ }^{\circ} \mathrm{C}\right)$. Isolated yield: 157 mg (53\%). ${ }^{1} \mathrm{H}-\mathrm{NMR}\left(395 \mathrm{MHz}, \mathrm{CDCl}_{3}\right) \delta: 0.05$ (s, 6H, - $\mathrm{SiMe}_{2}$ ), 0.06 (s, 9H, - $\mathrm{SiMe}_{3}$ ), 0.51-0.55 (m, 2H, $\left.-\mathrm{CH}_{2} \mathrm{Si}\right), 1.60-1.68\left(\mathrm{~m}, 2 \mathrm{H},-\mathrm{CH}_{2^{-}}\right), 3.44\left(\mathrm{t}, J=7.3 \mathrm{~Hz}, 2 \mathrm{H}, \mathrm{O}-\mathrm{CH}_{2}-\right), 4.51\left(\mathrm{~s}, 2 \mathrm{H}, \mathrm{O}-\mathrm{CH}_{2}-\mathrm{Ar}\right), 7.27-7.37$ (m, $5 \mathrm{H}$, $\mathrm{Ar}-\mathrm{H}) .{ }^{13} \mathrm{C}-\mathrm{NMR}\left(99 \mathrm{MHz}, \mathrm{CDCl}_{3}\right) \delta: 0.4,2.0,14.5,23.7,72.9,73.3,127.5,127.7,128.4,138.8 .{ }^{29} \mathrm{Si}-\mathrm{NMR}$ $\left(119 \mathrm{MHz}, \mathrm{CDCl}_{3}\right) \delta: 7.40,7.67$. HRMS (EI) calcd for $\left[\mathrm{C}_{15} \mathrm{H}_{28} \mathrm{O}_{2} \mathrm{Si}_{2}\right]-\mathrm{Me}: 281.1393$. found 281.1394.

\section{General Procedure for the hydrosilylation of alkenes with MD'M by the $\mathrm{Co}(\mathrm{OPv})_{2}$ / CNAd catalyst (Table} S9)

In a $6 \mathrm{~mL}$ glass vial were placed $\mathrm{Co}(\mathrm{OPv})_{2}(0.01 \mathrm{mmol}$ or $0.10 \mathrm{mmol}, 1 \mathrm{~mol} \%)$ and CNAd $(0.03 \mathrm{mmol}$ or 0.30 mmol, $3 \mathrm{~mol} \%)$, then the mixture of alkene $(1 \mathrm{mmol}$ or $10 \mathrm{mmol})$ and MD'M (1.3 mmol or $13 \mathrm{mmol})$ was added. The resulting mixture was stirred at $80^{\circ} \mathrm{C}$ for the time indicated in the table. After cooling to room temperature, the conversion of alkenes and the yield of the product were determined by ${ }^{1} \mathrm{H}-\mathrm{NMR}$ spectroscopy using anisole as the internal standard. The products were isolated by distillation under a reduced pressure. 
Table S9. Hydrosilylation of alkenes with MD’M catalyzed the $\mathrm{Co}(\mathrm{OPv})_{2} / \mathrm{CNAd}$ catalyst.

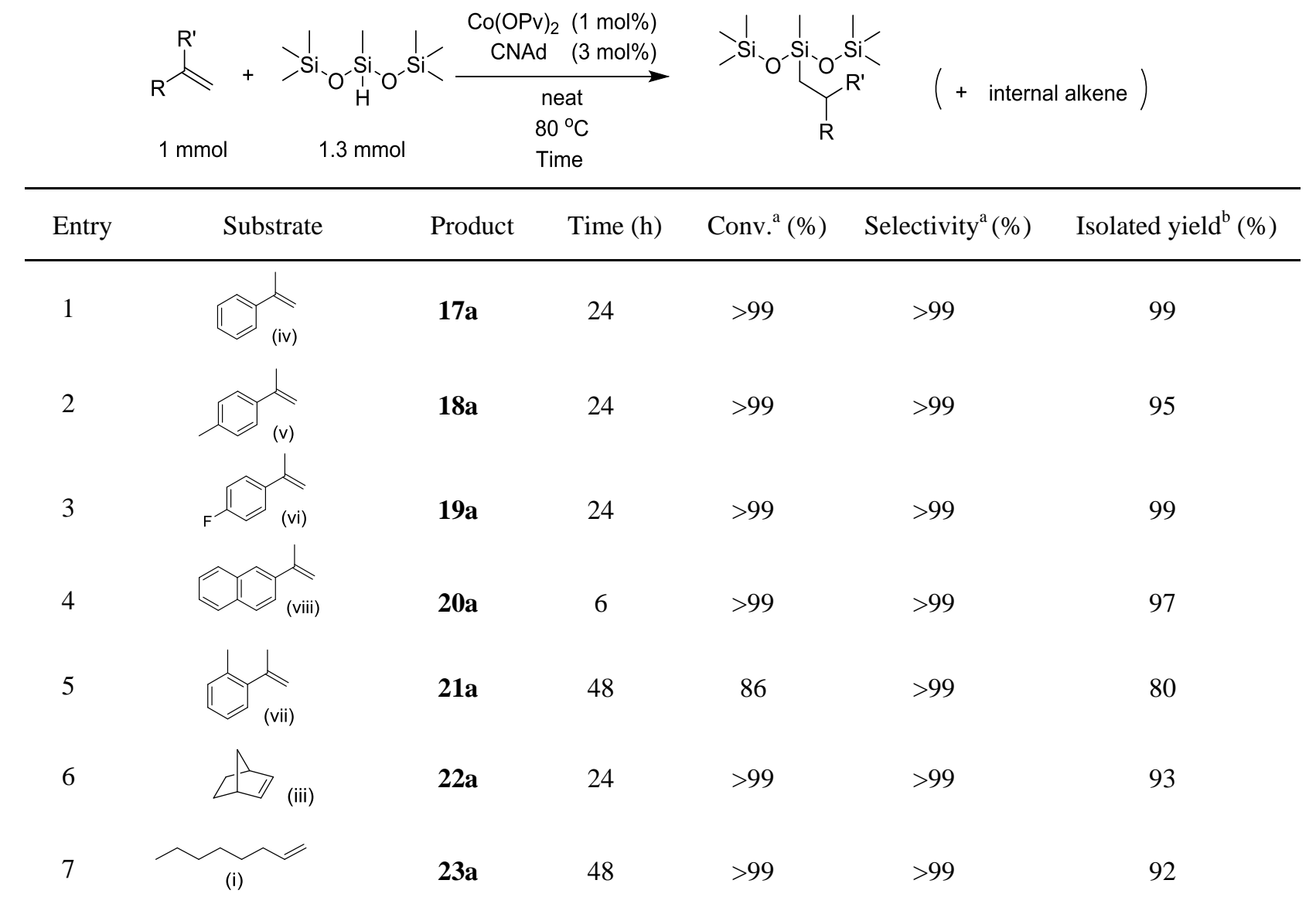

${ }^{\mathrm{a}}$ Conversion, selectivity, and the selectivity of the product were determined by ${ }^{1} \mathrm{H}$ NMR of the crude products. ${ }^{\mathrm{b}}$ The products 17a $\sim 23 \mathrm{a}$ were isolated by distillation.

\section{1,1,1-3-5,5,5-heptamethyl-3-(2-phenylpropyl)trisiloxane (17a)}

I I I The product 17a was obtained from the reaction of $\alpha$-methylstyrene (130 $\mu \mathrm{L} 1.0 \mathrm{mmol})$ with MD'M (353 $\mu \mathrm{L} 1.3 \mathrm{mmol})$ for $24 \mathrm{~h}$ at $80{ }^{\circ} \mathrm{C}$ catalyzed by $\mathrm{Co}(\mathrm{OPv})_{2}(3 \mathrm{mg}, 0.01$ mmol) and CNAd ( $5 \mathrm{mg}, 0.03 \mathrm{mmol}$ ) (Table $\mathrm{S} 9$, entry 1 ). The product was isolated by distillation $\left(8 \mathrm{~Pa}, 70-75{ }^{\circ} \mathrm{C}\right)$. Isolated yield : $340 \mathrm{mg}(99 \%) .{ }^{1} \mathrm{H}-\mathrm{NMR}\left(395 \mathrm{MHz}, \mathrm{CDCl}_{3}\right)$ $\delta:-0.12$ (s, 3H, -SiMe-), 0.07 (s, 9H, -SiMe 3 ), 0.09 (s, 9H, -SiMe $), 0.82-0.94$ (m, 2H, $-\mathrm{CH}_{2} \mathrm{Si}$ ), 1.28 (d, J=6.8 Hz, 3H, - $\mathrm{CH}_{3}$ ), 2.92 (sext, J=6.8 Hz, 1H, -CH-), 7.16 (t, J=6.8 Hz, 1H, Ar-H), 7.21 (d, $J=6.8 \mathrm{~Hz}, 2 \mathrm{H}, \mathrm{Ar}-\mathrm{H}), 7.27$ (t, $J=6.8 \mathrm{~Hz}, 2 \mathrm{H}, \mathrm{Ar}-\mathrm{H}) .{ }^{13} \mathrm{C}-\mathrm{NMR}\left(99 \mathrm{MHz}, \mathrm{CDCl}_{3}\right) \delta: 0.6,1.8,1.9,25.5,27.7,35.3$, 125.6, 126.6, 128.3, 150.1. ${ }^{29} \mathrm{Si}-\mathrm{NMR}\left(119 \mathrm{MHz}, \mathrm{CDCl}_{3}\right) \delta:-23.01,6.98,7.13$. HRMS (EI) calcd for $\left[\mathrm{C}_{16} \mathrm{H}_{32} \mathrm{O}_{2} \mathrm{Si}_{3}\right]: 340.1710$. found 340.1711 . 


\section{1,1,1-3-5,5,5-heptamethyl-3-(2- (4-tolyl)propyl)trisiloxane (18a)}

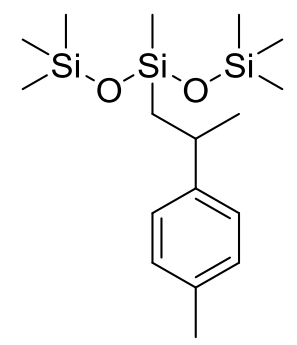

The product 18a was obtained from the reaction of 4-isopropenyltoluene (145 $\mu \mathrm{L} 1.0$ mmol) with MD'M (353 $\mu \mathrm{L} 1.3 \mathrm{mmol})$ for $24 \mathrm{~h}$ at $80{ }^{\circ} \mathrm{C}$ catalyzed by $\mathrm{Co}(\mathrm{OPv})_{2}(3 \mathrm{mg}$, $0.01 \mathrm{mmol}$ ) and CNAd (5 mg, $0.03 \mathrm{mmol}$ ) (Table $\mathrm{S} 9$, entry 2). The product was isolated by distillation $\left(8 \mathrm{~Pa}, 90-95{ }^{\circ} \mathrm{C}\right.$ ). Isolated yield : $337 \mathrm{mg}$ (95\%). ${ }^{1} \mathrm{H}-\mathrm{NMR}(395 \mathrm{MHz}$, $\left.\mathrm{CDCl}_{3}\right) \delta:-0.12$ (s, 3H, -SiMe-), 0.07 (s, 9H, -SiMe $\left.{ }_{3}\right), 0.08$ (s, 9H, -SiMe 3 ), 0.80-0.91 (m, $2 \mathrm{H},-\mathrm{CH}_{2} \mathrm{Si}$ ), 1.25 (d, J=6.8 Hz, 3H, $-\mathrm{CH}_{3}$ ), 2.31 (s, 3H, $-\mathrm{OMe}$ ), 2.88 (sext, J=6.8 Hz, $1 \mathrm{H}$, -CH-), 7.06-7.11 (m, 4H, Ar-H). ${ }^{13} \mathrm{C}-\mathrm{NMR}\left(99 \mathrm{MHz}, \mathrm{CDCl}_{3}\right) \delta: 0.6,1.8,1.9,20.9,25.5,27.8,34.9,126.4$, 128.9, 135.0, 147.2. ${ }^{29} \mathrm{Si}-\mathrm{NMR}\left(119 \mathrm{MHz}, \mathrm{CDCl}_{3}\right) \delta:-22.90,6.94,7.05$. HRMS (EI) calcd for $\left[\mathrm{C}_{17} \mathrm{H}_{34} \mathrm{O}_{2} \mathrm{Si}_{3}\right]$ : 354.1867. found 354.1864.

\section{1,1,1-3-5,5,5-heptamethyl-3-(2- (4-fluorophenyl)propyl)trisiloxane (19a)}<smiles>CC(C[Si](C)(C)O[Si](C)(C)C)c1ccc(F)cc1</smiles>

The product 19a was obtained from the reaction of 4-fluoroisopropenylbenzene (134 $\mu \mathrm{L}$ $1.0 \mathrm{mmol})$ with MD'M (353 $\mu \mathrm{L} 1.3 \mathrm{mmol})$ for $24 \mathrm{~h}$ at $80{ }^{\circ} \mathrm{C}$ catalyzed by $\mathrm{Co}(\mathrm{OPv})_{2}(3 \mathrm{mg}$, $0.01 \mathrm{mmol}$ ) and CNAd (5 mg, $0.03 \mathrm{mmol}$ ) (Table S9, entry 3). The product was isolated by distillation $\left(8 \mathrm{~Pa}, 90-95{ }^{\circ} \mathrm{C}\right.$ ). Isolated yield : $356 \mathrm{mg}(99 \%) .{ }^{1} \mathrm{H}-\mathrm{NMR}(395 \mathrm{MHz}$, $\left.\mathrm{CDCl}_{3}\right) \delta:-0.13$ (s, 3H, $-\mathrm{SiMe}_{3}$ ), 0.07 (s, 9H, -SiMe 3 ), 0.08 (s, 9H, -SiMe $), 0.79-0.90$ (m, $2 \mathrm{H},-\mathrm{CH}_{2} \mathrm{Si}$ ), 1.24 (d, J=6.8 Hz, 3H, $\left.-\mathrm{CH}_{3}\right), 2.91$ (sext, J=6.8 Hz, 1H, -CH-), 6.93-6.97 (m, $2 \mathrm{H}, \mathrm{Ar}-\mathrm{H}), 7.13-7.17(\mathrm{~m}, 2 \mathrm{H}, \mathrm{Ar}-\mathrm{H}) .{ }^{13} \mathrm{C}-\mathrm{NMR}\left(99 \mathrm{MHz}, \mathrm{CDCl}_{3}\right) \delta: 0.6,1.8,1.8,25.8,27.9,34.7,114.9(\mathrm{~d}$, $\left.J_{C-F}=21.3\right), 127.9\left(\mathrm{~d}, J_{C-F}=7.4\right), 145.6\left(\mathrm{~d}, J_{C-F}=3.3\right), 161.1\left(\mathrm{~d}, J_{C-F}=242.8\right) .{ }^{19} \mathrm{~F}-\mathrm{NMR}\left(565 \mathrm{MHz}, \mathrm{CDCl}_{3}\right) \delta$ : -119.05. ${ }^{29} \mathrm{Si}-\mathrm{NMR}\left(119 \mathrm{MHz}, \mathrm{CDCl}_{3}\right) \delta: 23.28,7.13,7.25$. HRMS (EI) calcd for $\left[\mathrm{C}_{16} \mathrm{H}_{31} \mathrm{FO}_{2} \mathrm{Si}_{3}\right]: 358.1616$ found 358.1618 .

\section{1,1,1-3-5,5,5-heptamethyl-3-(2- (2-naphthyl) propyl)trisiloxane (20a)}<smiles>CC(C[Si](C)(C)O[Si](C)(C)C)c1ccc2ccccc2c1</smiles>

The product 20a was obtained from the reaction of 2-isopropenylnaphthalene (168 $\mathrm{mg} 1.0$ mmol) with MD’M $(353 \mu \mathrm{L} 1.3 \mathrm{mmol})$ for $6 \mathrm{~h}$ at $80{ }^{\circ} \mathrm{C}$ catalyzed by $\mathrm{Co}(\mathrm{OPv})_{2}(3 \mathrm{mg}, 0.01$ mmol) and CNAd (5 mg, $0.03 \mathrm{mmol}$ ) (Table S9, entry 4). The product was isolated by alumina column chromatography (AcOEt : hexane, 1:10). Isolated yield : $377 \mathrm{mg}(97 \%)$. ${ }^{1} \mathrm{H}-\mathrm{NMR}\left(395 \mathrm{MHz}, \mathrm{CDCl}_{3}\right.$ ) $\delta:-0.09$ (s, 3H, -SiMe-), 0.07(s, 9H, -SiMe $), 0.09$ (s, 9H, $-\mathrm{SiMe}_{3}$ ), 0.92-1.04 (m, 2H, - $\mathrm{CH}_{2} \mathrm{Si}$ ), 1.37 (d, J=6.8 Hz, 3H, - $\mathrm{CH}_{3}$ ), 3.11 (sext, $J=6.8 \mathrm{~Hz}, 1 \mathrm{H}$, -CH-), 7.38-7.46 (m, 3H, Ar-H), 7.62 (s, 1H, Ar-H), 7.76-7.80 (m, 3H, Ar-H). ${ }^{13} \mathrm{C}-\mathrm{NMR}$ $\left(99 \mathrm{MHz}, \mathrm{CDCl}_{3}\right) \delta: 0.7,1.9,1.9,25.4,27.7,35.5,124.4,124.9,125.7,127.6,127.9,132.1,133.7,147.6$. ${ }^{29} \mathrm{Si}-\mathrm{NMR}\left(119 \mathrm{MHz}, \mathrm{CDCl}_{3}\right) \delta:-22.97,7.13,7.25$. HRMS (EI) calcd for $\left[\mathrm{C}_{20} \mathrm{H}_{34} \mathrm{O}_{2} \mathrm{Si}_{3}\right]:$ 390.1867. found 390.1866 . 


\section{1,1,1-3-5,5,5-heptamethyl-3-(2- (2-tolyl)propyl)trisiloxane (21a)}

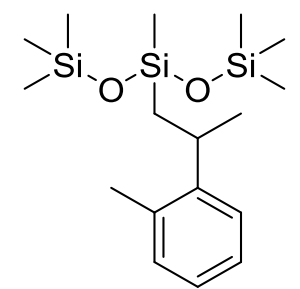

The product 21a was obtained from the reaction of 2-isopropenyltoluene (145 $\mu \mathrm{L} 1.0$ mmol) with MD'M (353 $\mu \mathrm{L} 1.3 \mathrm{mmol})$ for $48 \mathrm{~h}$ at $80{ }^{\circ} \mathrm{C}$ catalyzed by $\mathrm{Co}(\mathrm{OPv})_{2}(3 \mathrm{mg}$, $0.01 \mathrm{mmol}$ ) and CNAd (5 mg, $0.03 \mathrm{mmol}$ ) (Table $\mathrm{S} 9$, entry 5). The product was isolated by distillation $\left(8 \mathrm{~Pa}, 90-95{ }^{\circ} \mathrm{C}\right.$ ). Isolated yield : $284 \mathrm{mg}(80 \%) .{ }^{1} \mathrm{H}-\mathrm{NMR}(395 \mathrm{MHz}$, $\left.\mathrm{CDCl}_{3}\right) \delta:-0.07$ (s, 3H, -SiMe-), 0.08 (s, 18H, -SiMe 3 ), 0.79-0.90 (m, 2H, $\left.-\mathrm{CH}_{2} \mathrm{Si}\right), 1.23$ (d, $J=6.8 \mathrm{~Hz}, 3 \mathrm{H},-\mathrm{CH}_{3}$ ), 2.33 (s, Ar-Me), 3.19 (sext, $J=6.8 \mathrm{~Hz}, 1 \mathrm{H},-\mathrm{CH}-$ ), 7.03-7.26 (m, 4H, Ar-H). ${ }^{13} \mathrm{C}-\mathrm{NMR}$ $\left(99 \mathrm{MHz}, \mathrm{CDCl}_{3}\right) \delta: 0.6,1.9,19.4,24.3,27.0,29.8,125.2,125.3,126.2,130.1,134.4,148.2 .{ }^{29} \mathrm{Si}-\mathrm{NMR}$ $\left(119 \mathrm{MHz}, \mathrm{CDCl}_{3}\right) \delta:-22.78,7.05,7.29$. HRMS (EI) calcd for $\left[\mathrm{C}_{17} \mathrm{H}_{34} \mathrm{O}_{2} \mathrm{Si}_{3}\right]$ : 354.1867. found 354.1867.

\section{1-(bicyclo[2.2.1]heptan-2-yl)-1,1,1-3-5,5,5-heptamethyltrisiloxane (22a)}

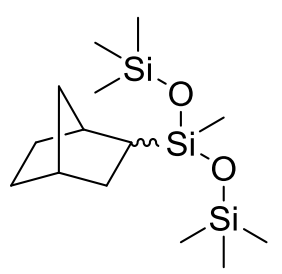

The product 22a was obtained from the reaction of 2-norbornene (94 $\mathrm{mg} 1.0 \mathrm{mmol}$ ) with MD'M (353 $\mu \mathrm{L} 1.3 \mathrm{mmol})$ for $24 \mathrm{~h}$ at $80{ }^{\circ} \mathrm{C}$ catalyzed by $\mathrm{Co}(\mathrm{OPv})_{2}(3 \mathrm{mg}, 0.01 \mathrm{mmol})$ and CNAd ( $5 \mathrm{mg}, 0.03 \mathrm{mmol}$ ) (Table S9, entry 6). The product was isolated by distillation (6 $\mathrm{Pa}, 60-65{ }^{\circ} \mathrm{C}$ ). Isolated yield : $295 \mathrm{mg}(93 \%)$. GLC (TC-1 (30 m) column temperature 160

${ }^{\circ} \mathrm{C}$ ) $\mathrm{t}_{\mathrm{R}} 10.0 \mathrm{~min}$ (endo), $10.4 \mathrm{~min}$ (exo). exo:endo = 91.9: 8.1. ${ }^{1} \mathrm{H}-\mathrm{NMR}\left(395 \mathrm{MHz}, \mathrm{CDCl}_{3}\right)$ $\delta$ : -0.04 (s, 3H, -SiMe-, exo), 0.01 (s, 0.21H), 0.08 (s, 18H, -SiMe 3 , exo), 0.10 (s, 0.97H), 0.45 (t, J=8.2 Hz, 1H, -CH-Si, exo), 0.80-0.84 (m, 0.08H), 1.07-1.09 (m, 1H, exo), 1.10-1.13 (m, 0.07H), 1.14-1.22 (m, 2.19H, exo and endo), $1.25-1.27(\mathrm{~m}, 0.11 \mathrm{H}), 1.27-1.33(\mathrm{~m}, 2 \mathrm{H}$, exo $), 1.33-1.35(\mathrm{~m}, 0.5 \mathrm{H}), 1.40-1.43(\mathrm{~m}, 0.7 \mathrm{H}), 1.43-1.48$ (m, 1H, exo), 1.48-1.55 (m, 2H, exo), 1.57-1.64 (m, 0.20H), 2.20-2.22 (m, 2H, exo), 2.22-2.30 (m, 0.20H). (Assignment of the exo- and endo- isomer was performed by ${ }^{1} \mathrm{H}-{ }^{1} \mathrm{H}$ COSY spectrum (Figure S26-4)). ${ }^{13} \mathrm{C}-\mathrm{NMR}$ $\left(99 \mathrm{MHz}, \mathrm{CDCl}_{3}\right) \delta:-1.5(\mathrm{exo}),-1.4(\mathrm{exo}), 1.9(\mathrm{exo}), 29.0(\mathrm{exo}), 30.2(\mathrm{exo}), 30.2(\mathrm{exo}), 31.6(\mathrm{exo}), 33.9(\mathrm{exo})$, 36.7(exo), 36.7(exo), 37.3(exo), 37.3(exo), 37.6(exo). ${ }^{29} \mathrm{Si}-\mathrm{NMR}\left(119 \mathrm{MHz}, \mathrm{CDCl}_{3}\right) \delta:-23.55(\mathrm{exo}), 6.63(\mathrm{exo})$. HRMS (EI) calcd for $\left[\mathrm{C}_{14} \mathrm{H}_{32} \mathrm{O}_{2} \mathrm{Si}_{3}\right]$ : 316.1710. found 316.1709.

\section{1,1,1-3,3-pentamethyl-3-octyldisiloxane (23a)}

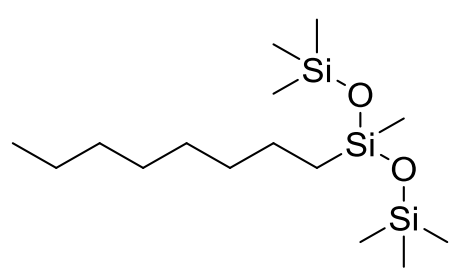

The product 23a as obtained from the reaction of 1-octene (1.122 g 10.0 mmol) with PMDS (2.895 g $13.0 \mathrm{mmol})$ for $48 \mathrm{~h}$ at $80{ }^{\circ} \mathrm{C}$ catalyzed by $\mathrm{Co}(\mathrm{OPv})_{2}(26 \mathrm{mg}, 0.10 \mathrm{mmol})$ and CNAd $(48 \mathrm{mg}, 0.30 \mathrm{mmol})$ (Table S9, entry 7). The product was isolated by distillation $\left(8 \mathrm{~Pa}, 65-70{ }^{\circ} \mathrm{C}\right)$. Isolated yield : $3.073 \mathrm{~g}(92 \%) .{ }^{1} \mathrm{H}-\mathrm{NMR}\left(395 \mathrm{MHz}, \mathrm{CDCl}_{3}\right) \delta:-0.01$ (s, 3H, -SiMe), 0.08 (s, 18H, - $\mathrm{OSiMe}_{3}$ ), 0.42-0.46 (m, $\left.2 \mathrm{H},-\mathrm{CH}_{2} \mathrm{Si}\right), 0.88$ (t, $J=6.8 \mathrm{~Hz}, 3 \mathrm{H}$, $\left.-\mathrm{CH}_{3}\right), 1.22-1.38\left(\mathrm{~m}, 12 \mathrm{H},-\mathrm{CH}_{2}-\right) .{ }^{13} \mathrm{C}-\mathrm{NMR}\left(99 \mathrm{MHz}, \mathrm{CDCl}_{3}\right) \delta:-0.2,1.9,14.1,17.7,22.8,23.2,29.4,29.4$, 32.0, 33.3, 33.3. ${ }^{29} \mathrm{Si}-\mathrm{NMR}\left(119 \mathrm{MHz}, \mathrm{CDCl}_{3}\right) \delta:-21.24,6.71$. HRMS (EI) calcd for $\left[\mathrm{C}_{14} \mathrm{H}_{35} \mathrm{O}_{2} \mathrm{Si}_{3}\right]-\mathrm{Me}$ : 319.1945. found 319.1944. 


\section{Screening of the co-catalyst for the hydrosilylation of styrene with PMDS by $\mathrm{Fe}(\mathrm{OPv})_{2}$ / CNAd catalyst}

(Table S10 and S11)

A procedure for the reactions in Table S10: In a $20 \mathrm{~mL}$ Schlenk tube, $\mathrm{Fe}(\mathrm{OPv})_{2}(3 \mathrm{mg} 0.01 \mathrm{mmol})$ and CNAd (3 $\mathrm{mg}, 0.02 \mathrm{mmol})$ were dissolved in THF $(0.10 \mathrm{~mL})$. The co-catalyst (boranes or hydrosilanes) was added into this solution at room temperature. The mixture was stirred at room temperature for $1 \mathrm{~h}$, styrene $(115 \mu \mathrm{L}, 1.0$ mmol) and PMDS ( $254 \mu \mathrm{L} 1.3 \mathrm{mmol})$ were added. The resulting mixture was stirred at $25^{\circ} \mathrm{C}$ for $3 \mathrm{~h}$. The yield of the product 1a was determined by ${ }^{1} \mathrm{H}-\mathrm{NMR}$ spectroscopy using anisole as the internal standard.

Table S10. Hydrosilylation of styrene with PMDS by the $\mathrm{Fe}(\mathrm{OPv})_{2} /$ CNAd catalyst in the presence of borane or hydrosilane co-catalyst.

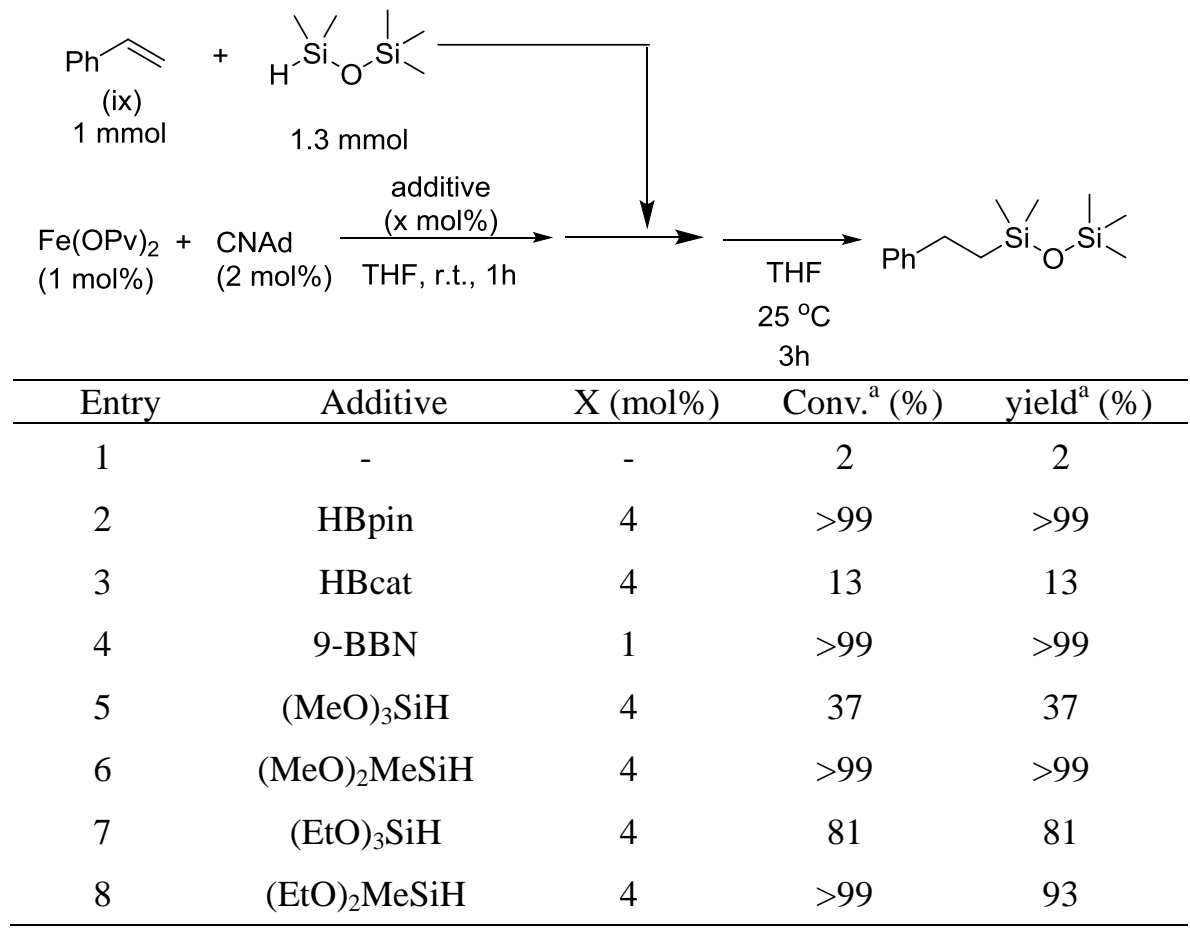

HBpin:pinacolborane, HBcat:catecholborane, 9-BBN:9-borabicyclo[3.3.1]nonane dimer. ${ }^{\text {a }}$ Conversion and the yield of the product were determined by both ${ }^{1} \mathrm{H}$ NMR and GC spectra of the crude products.

A procedure for the reactions in Table S11: In a $20 \mathrm{~mL}$ Schlenk tube, $\mathrm{Fe}(\mathrm{OPv})_{2}(3 \mathrm{mg} 0.01 \mathrm{mmol})$, CNAd (3 $\mathrm{mg}, 0.02 \mathrm{mmol})$, styrene (115 $\mu \mathrm{L}, 1.0 \mathrm{mmol})$ and PMDS ( $254 \mu \mathrm{L} 1.3 \mathrm{mmol})$ were dissolved in THF (60 $\mu \mathrm{L}$ or $87 \mu \mathrm{L})$. A $1.0 \mathrm{M}$ THF solution of $\mathrm{EtMgBr}(40 \mu \mathrm{L}, 0.040 \mathrm{mmol})$ or a $1.0 \mathrm{M}$ hexane solution of $\mathrm{Et}_{3} \mathrm{Al}(13 \mu \mathrm{L}$, $0.013 \mathrm{mmol}$ ) were added into this solution at $-78{ }^{\circ} \mathrm{C}$. The resulting mixture was stirred at $25{ }^{\circ} \mathrm{C}$ for $3 \mathrm{~h}$. The yield of the product 1a was determined by ${ }^{1} \mathrm{H}-\mathrm{NMR}$ spectroscopy using anisole as the internal standard. 
Table S11. Hydrosilylation of styrene with PMDS by the $\mathrm{Fe}(\mathrm{OPv})_{2} / \mathrm{CNAd}$ catalyst in the presence of EtMgBr or $\mathrm{Et}_{3} \mathrm{Al}$ co-catalyst.

\begin{tabular}{|c|c|c|c|c|c|c|}
\hline $\begin{array}{l}\mathrm{Ph} \curvearrowright \\
1 \mathrm{(ix}) \\
1 \mathrm{mmol}\end{array}$ & 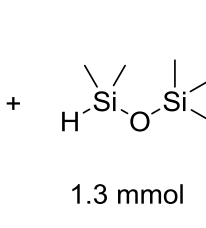 & $\begin{array}{l}\mathrm{Ee}(\mathrm{OPv})_{2}(1 \mathrm{~mol} \%) \\
\stackrel{\mathrm{CNAd} \quad(2 \mathrm{~mol} \%)}{\longrightarrow}\end{array}$ & $\begin{array}{c}\begin{array}{c}\text { additive } \\
\text { (x mol\%) }\end{array} \\
\begin{array}{c}\text { solvent } \\
-78^{\circ} \mathrm{C} \text { to } 25^{\circ} \mathrm{C} \\
3 \mathrm{~h}\end{array}\end{array}$ & 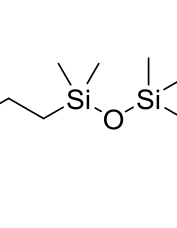 & & \\
\hline & Entry & Additive & Solvent & $\mathrm{X}(\mathrm{mol} \%)$ & Conv. $^{\text {a }}(\%)$ & $\operatorname{yield}^{\mathrm{a}}(\%)$ \\
\hline & 1 & - & THF & - & 2 & 2 \\
\hline & 2 & EtMgBr & THF & 4 & 9 & 9 \\
\hline & 3 & $\mathrm{Et}_{3} \mathrm{Al}$ & THF/hexane & 1.3 & $>99$ & $>99$ \\
\hline
\end{tabular}

${ }^{\mathrm{a}}$ Conversion and the yield of the product were determined by ${ }^{1} \mathrm{H}$ NMR spctrum of the crude products.

Screening of the co-catalyst for the hydrosilylation of $\alpha$-methylstyrene with PMDS by $\operatorname{Co}(\mathrm{OPv})_{2} / \mathrm{CNAd}$ catalyst (Table S12)

In a $6 \mathrm{~mL}$ glass vial, the mixture of $\mathrm{Co}(\mathrm{OPv})_{2}(3 \mathrm{mg} 0.01 \mathrm{mmol})$ and $\mathrm{CNAd}(3 \mathrm{mg}, 0.02 \mathrm{mmol})$ were dissolved in THF or DME $(0.10 \mathrm{~mL})$. The co-catalyst was added into this solution at room temperature. The obtained mixture was stirred at room temperature for $1 \mathrm{~h}$, then the mixture of $\alpha$-methylstyrene $(130 \mu \mathrm{L}, 1.0 \mathrm{mmol})$ and PMDS $(254 \mu \mathrm{L} 1.3 \mathrm{mmol})$ was added. The resulting mixture was stirred at $50{ }^{\circ} \mathrm{C}$ for $3 \mathrm{~h}$. The yield of the product $2 \mathbf{a}$ was determined by ${ }^{1} \mathrm{H}-\mathrm{NMR}$ spectroscopy using anisole as the internal standard.

Table S12. Hydrosilylation of $\alpha$-methylstyrene with PMDS catalyzed by $\mathrm{Co}(\mathrm{OPv})_{2}$ with CNAd in the presence of various co-catalysts

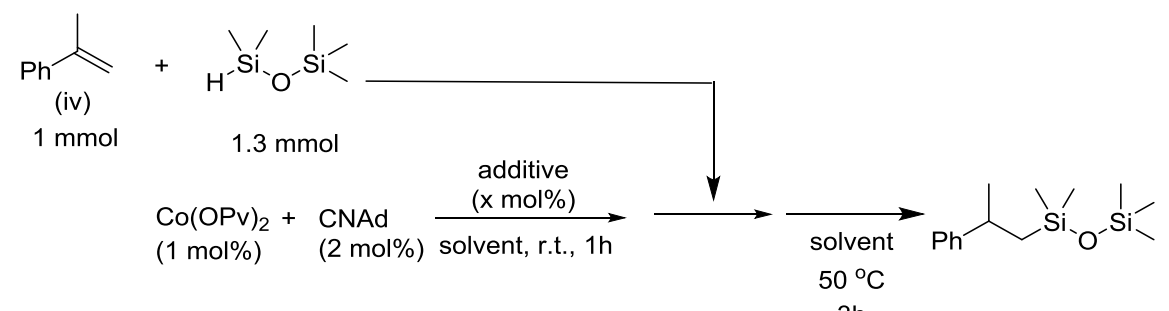

$3 \mathrm{~h}$

\begin{tabular}{cccccc}
\hline Entry & Additive & Solvent & $\mathrm{X}(\mathrm{mol} \%)$ & Conv. $^{\mathrm{a}}(\%)$ & Yield $^{\mathrm{a}}(\%)$ \\
\hline 1 & - & THF & - & 1 & 1 \\
2 & HBpin & DME & 4 & 10 & 10 \\
3 & HBcat & DME & 4 & 36 & 36 \\
4 & $9-\mathrm{BBN}$ & DME & 1 & 98 & 98 \\
5 & $(\mathrm{MeO})_{3} \mathrm{SiH}$ & THF & 4 & $>99$ & $>99$ \\
6 & $(\mathrm{MeO})_{2} \mathrm{MeSiH}$ & THF & 4 & 74 & 74 \\
7 & $(\mathrm{EtO})_{3} \mathrm{SiH}$ & THF & 4 & $>99$ & $>99$ \\
8 & $(\mathrm{EtO})_{2} \mathrm{MeSiH}$ & THF & 4 & $>99$ & $>99$ \\
\hline
\end{tabular}

${ }^{\mathrm{a}}$ Conversion and the yield of the product were determined by ${ }^{1} \mathrm{H}$ NMR and GC spectra of the crude products. 
Screening of metal halides in the iron and cobalt catalyzed hydrosilylation of styrene or $\alpha$-methylstyrene with PMDS in the presence of magnesium or borane co-catalysts (Table S13 and S14)

A procedure for the reactions in Table S13: In a $20 \mathrm{~mL}$ Schlenk tube, metal halides $(0.01 \mathrm{mmol})$ indicated in the tables, CNAd ( 2 or 3 eq. to the metal halide), styrene $(115 \mu \mathrm{L}, 1.0 \mathrm{mmol})$ or $\alpha$-methylstyrene $(130 \mu \mathrm{L}, 1.0$ mmol), and PMDS (254 $\mu \mathrm{L} 1.3 \mathrm{mmol})$ were dissolved in THF $(0.10 \mathrm{~mL})$. A THF solution (1.0 M) of EtMgBr was added into this solution at $-78{ }^{\circ} \mathrm{C}$. The resulting mixture was warmed to $25 \sim 50^{\circ} \mathrm{C}$, and stirred for the $3 \sim 6 \mathrm{~h}$ (see, details in the tables). The yield of the product 1a or $\mathbf{2 a}$ was determined by ${ }^{1} \mathrm{H}-\mathrm{NMR}$ spectroscopy using anisole as the internal standard.

A procedure for the reactions in Table S14: In a $20 \mathrm{~mL}$ Schlenk tube, an iron or cobalt halide (0.01 mmol), CNAd $\{3 \mathrm{mg}(0.02 \mathrm{mmol})$ or $5 \mathrm{mg}(0.03 \mathrm{mmol})\}$ were dissolved in THF $(0.10 \mathrm{~mL})$. The boranes were added into this solution at room temperature. The obtained mixture was stirred at room temperature for $1 \mathrm{~h}$, then the mixture of styrene $(115 \mu \mathrm{L}, 1.0 \mathrm{mmol})$ or $\alpha$-methylstyrene $(130 \mu \mathrm{L}, 1.0 \mathrm{mmol})$ and PMDS (254 $\mu \mathrm{L} 1.3 \mathrm{mmol})$ was added. The resulting mixture was stirred for desired time. The yield of the product 1a or $2 \mathbf{a}$ was determined by ${ }^{1} \mathrm{H}-\mathrm{NMR}$ spectroscopy using anisole as the internal standard.

Table S13. Hydrosilylation of styrene or $\alpha$-methylstyrene with PMDS catalyzed by metal halide with CNAd in the presence of EtMgBr.

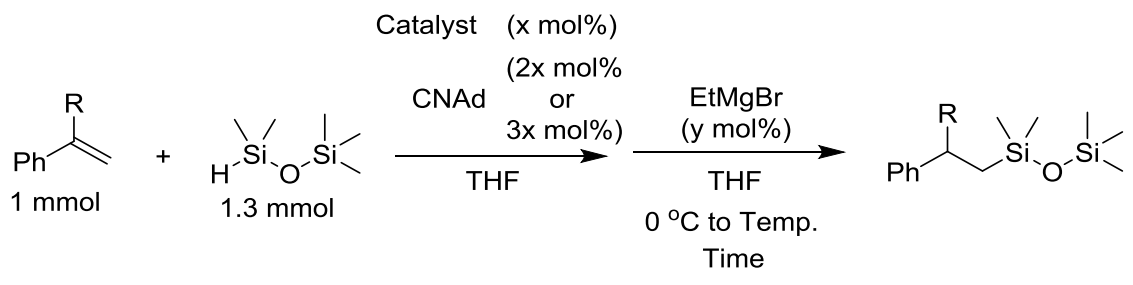

\begin{tabular}{cccccccc}
\hline Entry & $\mathrm{R}$ & $\begin{array}{c}\text { Catalyst } \\
(\mathrm{mol} \%)\end{array}$ & $\begin{array}{c}\text { Additive } \\
(\mathrm{mol} \%)\end{array}$ & Temp. $\left({ }^{\circ} \mathrm{C}\right)$ & Time (h) & Conv. $(\%)^{\mathrm{a}}$ & Yield $(\%)^{\mathrm{a}}$ \\
\hline 1 & $\mathrm{H} \mathrm{(ix)}$ & $\mathrm{FeCl}_{2}(3)$ & - & 50 & 6 & 0 & 0 \\
2 & $\mathrm{H} \mathrm{(ix)}$ & $\mathrm{FeCl}_{2}(3)$ & $\operatorname{EtMgBr}(6)$ & 50 & 6 & $>99$ & 94 \\
3 & $\mathrm{H} \mathrm{(ix)}$ & $\mathrm{FeBr}_{2}(3)$ & $\operatorname{EtMgBr}(6)$ & 50 & 6 & $>99$ & 96 \\
4 & $\mathrm{H} \mathrm{(ix)}$ & $\mathrm{FeCl}_{3}(3)$ & $\operatorname{EtMgBr}(9)$ & 50 & 6 & $>99$ & 95 \\
5 & $\mathrm{Me} \mathrm{(iv)}$ & $\mathrm{CoCl}_{2}(1)$ & - & 25 & 3 & 0 & 0 \\
6 & $\mathrm{Me} \mathrm{(iv)}$ & $\mathrm{CoCl}_{2}(1)$ & $\mathrm{EtMgBr}(4)$ & 25 & 3 & 52 & 51 \\
\hline
\end{tabular}

${ }^{\mathrm{a}}$ Conversion and the yield of the product were determined by ${ }^{1} \mathrm{H}$ NMR spectrum of the crude products. 
Table S14. Hydrosilylation of styrene or $\alpha$-methylstyrene with PMDS catalyzed by metal halide with CNAd in the presence of borane.

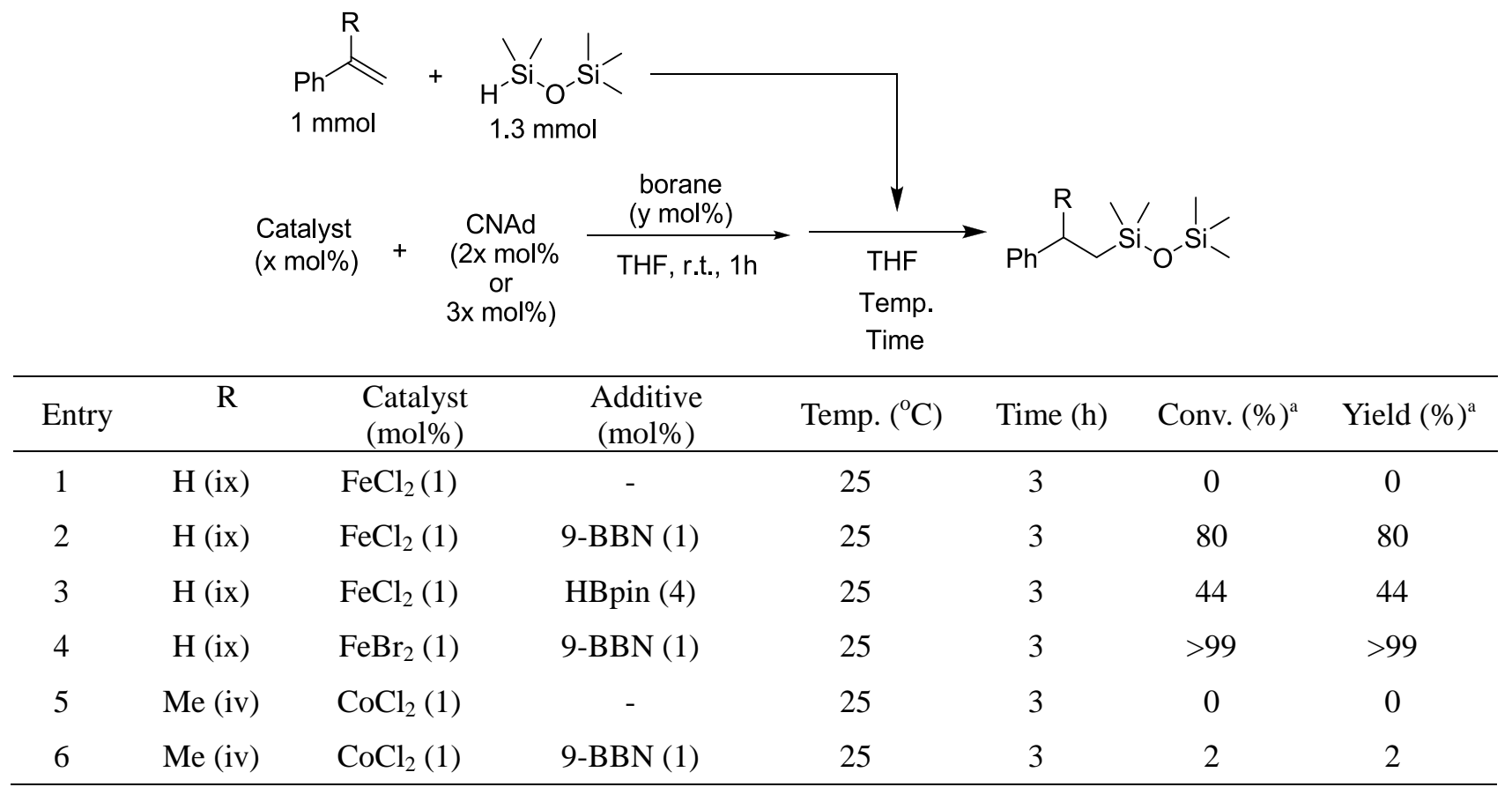

HBpin:pinacolborane, 9-BBN:9-borabicyclo[3.3.1]nonane dimer. ${ }^{\text {a }}$ Conversion and the yield of the product were determined by ${ }^{1} \mathrm{H}$ NMR spectrum of the crude products.

Hydrosilylations accelerated by borane or silane co-catalysts. Hydrosilylation of $\alpha$-methylstyrene with $P M D S$ by the $\mathrm{Co}(\mathrm{OPv})_{2}$ / CNAd catalyst in the presence of $(\mathrm{EtO})_{3} \mathrm{SiH}$ (Table 2, entry 2): In a $6 \mathrm{~mL}$ glass vial, $\mathrm{Co}(\mathrm{OPv})_{2}(3 \mathrm{mg} 0.01 \mathrm{mmol})$ and CNAd $(3 \mathrm{mg}, 0.02 \mathrm{mmol})$ were dissolved in DME $(0.10 \mathrm{~mL})$, then $(\mathrm{EtO})_{3} \mathrm{SiH}$ (7 $\mathrm{mg}, 0.04 \mathrm{mmol}$ ) was added into the solution. The mixture was stirred at room temperature for $1 \mathrm{hr}$, followed by the addition of the mixture of $\alpha$-methylstyrene $(1.18 \mathrm{~g}, 10.0 \mathrm{mmol})$ and PMDS $(1.93 \mathrm{~g} 13.0 \mathrm{mmol})$. The resulting mixture was stirred at $50{ }^{\circ} \mathrm{C}$ for $3 \mathrm{~h}$. The conversion of $\alpha$-methylstyrene (90\%) and the yield of the product $(90 \%)$ were determined by ${ }^{1} \mathrm{H}-\mathrm{NMR}$ spectroscopy using anisole as the internal standard. The product 2a was isolated by distillation under a reduced pressure ( $\left.8 \mathrm{~Pa}, 60-65^{\circ} \mathrm{C}\right)$. Isolated yield: $2.20 \mathrm{~g}(83 \%)$ Hydrosilylation of styrene with PMDS by the $\mathrm{Fe}(\mathrm{OPv})_{2}$ / CNAd catalyst in the presence of HBpin (Table 2, entry 4): In a $20 \mathrm{~mL}$ Schlenk tube, $\mathrm{Fe}(\mathrm{OPv})_{2}(3 \mathrm{mg} 0.01 \mathrm{mmol})$ and CNAd (3 mg, $\left.0.02 \mathrm{mmol}\right)$ were dissolved in DME $(0.50 \mathrm{~mL})$, then HBpin $(10 \mathrm{mg}, 0.08 \mathrm{mmol})$ was added into the solution. The mixture was stirred at room temperature for $1 \mathrm{hr}$, followed by the addition of the mixture of styrene $(5.21 \mathrm{~g}, 50.0 \mathrm{mmol})$ and PMDS $(9.65 \mathrm{~g}$ $65.0 \mathrm{mmol})$. The resulting mixture was stirred at $50{ }^{\circ} \mathrm{C}$ for $3 \mathrm{~h}$. The conversion of styrene (>99\%) and the yield of the product (>99\%) were determined by ${ }^{1} \mathrm{H}-\mathrm{NMR}$ spectroscopy using anisole as the internal standard. The product 1a was isolated by distillation under a reduced pressure ( $\left.8 \mathrm{~Pa}, 65-70{ }^{\circ} \mathrm{C}\right)$. Isolated yield: $12.13 \mathrm{~g}(95 \%)$ Hydrosilylation of styrene with PMDS by the $\mathrm{Fe}(\mathrm{OPv})_{2} / \mathrm{CNAd}$ catalyst in the presence of $(\mathrm{EtO})_{2} \mathrm{MeSiH}$ (Table 2, entry 5): In a $20 \mathrm{~mL}$ Schlenk tube, $\mathrm{Fe}(\mathrm{OPv})_{2}(3 \mathrm{mg} 0.01 \mathrm{mmol})$ and CNAd (3 mg, $\left.0.02 \mathrm{mmol}\right)$ were dissolved in DME $(0.10 \mathrm{~mL})$, then $(\mathrm{EtO})_{2} \mathrm{MeSiH}(14 \mathrm{mg}, 0.10 \mathrm{mmol})$ was added into the solution. The mixture was stirred at room temperature for $1 \mathrm{hr}$, followed by the addition of the mixture of styrene $(5.21 \mathrm{~g}, 50.0 \mathrm{mmol})$ and PMDS (9.65 g $65.0 \mathrm{mmol})$. The resulting mixture was stirred at $50{ }^{\circ} \mathrm{C}$ for $3 \mathrm{~h}$. The conversion of styrene 
(>99\%) and the yield of the product (>99\%) were determined by ${ }^{1} \mathrm{H}-\mathrm{NMR}$ spectroscopy using anisole as the internal standard. The product 1a was isolated by distillation under a reduced pressure $\left(8 \mathrm{~Pa}, 65-70{ }^{\circ} \mathrm{C}\right)$. Isolated yield: $12.17 \mathrm{~g}(96 \%)$

Hydrosilylation of allyl glycidyl ether with PMDS by the $\mathrm{Co}(\mathrm{OPv})_{2} / \mathrm{CNAd}$ catalyst in the presence of $(\mathrm{EtO})_{2} \mathrm{MeSiH}$ : In a $6 \mathrm{~mL}$ glass vial, $\mathrm{Co}(\mathrm{OPv})_{2}(3 \mathrm{mg}, 0.01 \mathrm{mmol})$ and CNAd (3 mg, $\left.0.02 \mathrm{mmol}\right)$ were dissolved in DME $(0.10 \mathrm{~mL})$, then $(\mathrm{EtO}){ }_{2} \mathrm{MeSiH}(8 \mu \mathrm{L}, 0.04 \mathrm{mmol})$ was added into the solution. The mixture was stirred at room temperature for $1 \mathrm{hr}$, followed by the addition of the mixture of PMDS ( $254 \mu \mathrm{L}, 1.3 \mathrm{mmol}$ ) and allyl glycidyl ether $(118 \mu \mathrm{L}, 1 \mathrm{mmol})$. The resulting mixture was stirred at room temperature for $23 \mathrm{~h}$. The conversion of allyl glycidyl ether (>99\%) and the yield of the product (>99\%) were determined by ${ }^{1} \mathrm{H}-\mathrm{NMR}$ spectroscopy using anisole as the internal standard. The product 9a was isolated by distillation under reduced pressure $\left(6 \mathrm{~Pa}, 60-65^{\circ} \mathrm{C}\right)$. Isolated yield: $87 \%(229 \mathrm{mg})$.

Hydrosilylation of 3-(2-methoxyethoxy)prop-1-ene with PMDS by the $\mathrm{Fe}(\mathrm{OPv})_{2} / \mathrm{CNAd}$ catalyst in the presence of $(\mathrm{EtO})_{2} \mathrm{MeSiH}$ : In a $6 \mathrm{~mL}$ glass vial, $\mathrm{Fe}(\mathrm{OPv})_{2}(8 \mathrm{mg}, 0.03 \mathrm{mmol})$ and CNAd $(10 \mathrm{mg}, 0.06 \mathrm{mmol})$ were dissolved in DME $(0.10 \mathrm{~mL})$, then $(\mathrm{EtO})_{2} \mathrm{MeSiH}(11 \mathrm{mg}, 0.08 \mathrm{mmol})$ was added into the solution. The mixture was stirred at room temperature for $1 \mathrm{hr}$, followed by the addition of the mixture of PMDS ( $254 \mu \mathrm{L}, 1.3 \mathrm{mmol}$ ) and 3-(2-methoxyethoxy)prop-1-ene $(116 \mathrm{mg}, 1 \mathrm{mmol})$. The resulting mixture was stirred at room temperature for $24 \mathrm{hrs}$. The conversion of 3-(2-methoxyethoxy)prop-1-ene (67\%) and the yield of the product (42\%) were determined by ${ }^{1} \mathrm{H}-\mathrm{NMR}$ spectroscopy using anisole as the internal standard. The product was isolated by alumina column chromatography (AcOEt : hexane, 1:10). Isolated yield : $65 \mathrm{mg}(24 \%)$. ${ }^{1} \mathrm{H}-\mathrm{NMR}$ (395MHz, $\left.\mathrm{CDCl}_{3}\right) \delta: 0.05\left(\mathrm{~s}, 6 \mathrm{H},-\mathrm{SiMe}_{2}\right), 0.06\left(\mathrm{~s}, 9 \mathrm{H},-\mathrm{OSiMe}_{3}\right), 0.46-0.52\left(\mathrm{~m}, 2 \mathrm{H},-\mathrm{CH}_{2} \mathrm{Si}\right), 1.58-1.66\left(\mathrm{~m}, 2 \mathrm{H},-\mathrm{CH}_{2}-\right)$, $3.39\left(\mathrm{~s}, 3 \mathrm{H},-\mathrm{OCH}_{3}\right), 3.41-3.45\left(\mathrm{~m}, 2 \mathrm{H},-\mathrm{CH}_{2}-\right), 3.53-3.60\left(\mathrm{~m}, 4 \mathrm{H},-\mathrm{CH}_{2^{-}}\right) .{ }^{13} \mathrm{C}-\mathrm{NMR}\left(99 \mathrm{MHz}, \mathrm{CDCl}_{3}\right) \delta: 0.2$, $1.9,14.2,23.4,59.1,69.9,72.0,74.3 .{ }^{29} \mathrm{Si}-\mathrm{NMR}\left(119 \mathrm{MHz}, \mathrm{CDCl}_{3}\right) \delta: 7.36,7.63$. HRMS (EI) calcd for $\left[\mathrm{C}_{11} \mathrm{H}_{28} \mathrm{O}_{3} \mathrm{Si}_{2}\right]-\mathrm{Me}: 249.1342$. found 249.1343.

Hydrosilylation of $\mathrm{CH}_{2}=\mathrm{CHCH}_{2}\left(\mathrm{OCH}_{2} \mathrm{CH}_{2}\right)_{n} \mathrm{OMe}(n=8)$ with PMDS by the $\mathrm{Fe}(\mathrm{OPv})_{2} / \mathrm{CNAd}$ catalyst in the presence of $(\mathrm{EtO})_{2} \mathrm{MeSiH}$ : In a $6 \mathrm{~mL}$ glass vial, the solution of the catalyst was prepared by dissolving the mixture of $\mathrm{Fe}(\mathrm{OPv})_{2}(8 \mathrm{mg}, 0.03 \mathrm{mmol})$ and CNAd $(10 \mathrm{mg}, 0.06 \mathrm{mmol})$ were dissolved in DME $(88 \mathrm{mg})$, $(\mathrm{EtO})_{2} \mathrm{MeSiH}(11 \mathrm{mg}, 0.08 \mathrm{mmol})$ was added into the solution, and then the mixture was stirred at room temperature for $1 \mathrm{hr}$. One-third of this solution was added to another $6 \mathrm{~mL}$ glass vial. The mixture of $\mathrm{CH}_{2}=\mathrm{CHCH}_{2}\left(\mathrm{OCH}_{2} \mathrm{CH}_{2}\right)_{\mathrm{n}} \mathrm{OMe}(\mathrm{n}=8)(43 \mathrm{mg}, 0.10 \mathrm{mmol})$ and PMDS $(19 \mathrm{mg}, 0.13 \mathrm{mmol})$ was added to this solution. The resulting mixture was stirred at room temperature for $24 \mathrm{~h}$. After cooling to room temperature, the conversion of alkene (89\%) and the yield of the product (62\%) were determined by ${ }^{1} \mathrm{H}-\mathrm{NMR}$ spectroscopy by using anisole as the internal standard. ${ }^{1} \mathrm{H}-\mathrm{NMR}\left(395 \mathrm{MHz}, \mathrm{CDCl}_{3}\right) \delta: 0.04$ (s, 6H, $\left.-\mathrm{SiMe}_{2}\right), 0.05$ (s, 9H, $\left.-\mathrm{OSiMe}_{3}\right), 0.46-0.51\left(\mathrm{~m}, 2 \mathrm{H},-\mathrm{CH}_{2} \mathrm{Si}\right), 1.53-1.65\left(\mathrm{~m}, 2 \mathrm{H},-\mathrm{CH}_{2}-\right), 3.37-3.41(\mathrm{~m}, 5 \mathrm{H}), 3.53-3.55(\mathrm{~m}, 2 \mathrm{H})$, 3.56-3.60 (m, 2H), 3.60-3.66 (br, 24H). HRMS (EI) calcd for $\left[\mathrm{C}_{25} \mathrm{H}_{56} \mathrm{O}_{10} \mathrm{Si}_{2}\right]-\mathrm{Me}$ : 557.3177. found 557.3177.

\section{Hydrosilylation of $\alpha$-methylstyrene with $\mathrm{Me}_{2} \mathrm{Si}(\mathrm{H}) \mathrm{O}\left(\mathrm{SiMe}_{2} \mathrm{O}\right)_{n} \mathrm{Si}(\mathrm{H}) \mathrm{Me}_{2}$ by the $\mathrm{Co}(\mathrm{OPv})_{2} / \mathrm{CNAd}$ catalyst}

(Table 3, entry 1 in the manuscript). In a $6 \mathrm{~mL}$ glass vial, $\mathrm{Co}(\mathrm{OPv})_{2}(3 \mathrm{mg}, 0.01 \mathrm{mmol})$ and CNAd (3 mg, $0.02 \mathrm{mmol})$ were dissolved in $\mathrm{DME}(0.1 \mathrm{~mL})$. Then, $\mathrm{Me}_{2} \mathrm{Si}(\mathrm{H}) \mathrm{O}\left(\mathrm{SiMe}_{2} \mathrm{O}\right)_{\mathrm{n}} \mathrm{Si}(\mathrm{H}) \mathrm{Me}_{2}(\mathrm{n}=$ ca. 27, determined by 
${ }^{1} \mathrm{H}$ NMR) (1.07 mg, ca $1.0 \mathrm{mmol}$ for $\left.\mathrm{Si}-\mathrm{H}\right)$ and $\alpha$-methylstyrene $(169 \mu \mathrm{L}, 1.3 \mathrm{mmol})$ were added, and the resulting mixture was stirred at $80{ }^{\circ} \mathrm{C}$ for $24 \mathrm{~h}$. The mixture was cooled to room temperature, and the conversion of hydrosilane (84\%) and the yield of the product (84\%) were determined by ${ }^{1} \mathrm{H}-\mathrm{NMR}$ spectroscopy using anisole as the internal standard. The resulting mixture was passed through a pad of $\mathrm{Al}_{2} \mathrm{O}_{3}$. The filtrate was dried under vacuum, then the product $\mathbf{2 4 a}$ was obtained in $77 \%$ yield $(917 \mathrm{mg})$.

Hydrosilylation of $\alpha$-methylstyrene with $\mathrm{Me}_{2} \mathrm{Si}(\mathrm{H}) \mathrm{O}\left(\mathrm{SiMe}_{2} \mathrm{O}\right)_{n} \mathrm{Si}(\mathrm{H}) \mathrm{Me}_{2}$ by the $\mathrm{Co}(\mathrm{OPv})_{2} / \mathrm{CNAd}$ catalyst in the presence of $(\mathrm{EtO})_{3} \mathrm{SiH}$ (Table 3, entry 2 in the manuscript). In a $6 \mathrm{~mL}$ glass vial, $\mathrm{Co}(\mathrm{OPv})_{2}(3 \mathrm{mg}$, $0.01 \mathrm{mmol})$ and CNAd $(3 \mathrm{mg}, 0.02 \mathrm{mmol}),(\mathrm{EtO}){ }_{3} \mathrm{SiH}(7 \mathrm{mg}, 0.04 \mathrm{mmol})$ were dissolved in DME $(0.1 \mathrm{~mL})$, then the mixture was stirred at room temperature for $1 \mathrm{~h}$. Then $\mathrm{Me}_{2} \mathrm{Si}(\mathrm{H}) \mathrm{O}\left(\mathrm{SiMe}_{2} \mathrm{O}\right)_{n} \mathrm{Si}\left(\mathrm{H}_{)}\right) \mathrm{Me}_{2}(\mathrm{n}=$ ca. 27 , determined by ${ }^{1} \mathrm{H}$ NMR) (1.07 mg, ca $1.0 \mathrm{mmol}$ for $\left.\mathrm{Si}-\mathrm{H}\right)$ and $\alpha$-methylstyrene (169 $\left.\mu \mathrm{L}, 1.3 \mathrm{mmol}\right)$ were added, and the resulting mixture was stirred at $50{ }^{\circ} \mathrm{C}$ for $24 \mathrm{~h}$. The mixture was cooled to room temperature, and the conversion of hydrosilane (>99\%) and the yield of the product (>99\%) were determined by ${ }^{1} \mathrm{H}-\mathrm{NMR}$ spectroscopy using anisole as the internal standard. The resulting mixture was passed through a pad of $\mathrm{Al}_{2} \mathrm{O}_{3}$. The filtrate was dried under vacuum, then the product $\mathbf{2 4 a}$ was obtained in $99 \%$ yield $(1.17 \mathrm{~g})$.

${ }^{1} \mathrm{H}-\mathrm{NMR}$ (395MHz, CDCl $)$ ) $\delta$ :-0.06 (s, 3H, -SiMe-), -0.03 (s, 3H, -SiMe-), 0.04 (s, 9H, -SiMe 3 ), 0.07 (s, 128H, - $\mathrm{SiMe}_{2}$ ), 0.92-1.02 (m, 4H, - $\mathrm{CH}_{2} \mathrm{Si}$ ), 1.28 (d, J=6.8 Hz, 6H, - $\mathrm{CH}_{3}$ ), 2.92 (sext, $J=6.8 \mathrm{~Hz}, 2 \mathrm{H},-\mathrm{CH}-$ ), 7.13-7.21 (m, 6H, Ar-H), 7.24-7.27 (m, 4H, Ar-H). ${ }^{13} \mathrm{C}-\mathrm{NMR}\left(99 \mathrm{MHz}, \mathrm{CDCl}_{3}\right) \delta: 0.7,1.0,1.2,1.2,1.4,26.0,28.4,35.6$, 125.7, 126.6, 128.3, 149.9. ${ }^{29} \mathrm{Si}-\mathrm{NMR}\left(119 \mathrm{MHz}, \mathrm{CDCl}_{3}\right) \delta:-22.12,-21.89,-21.55,6.59$.

ICP-MS: ICP-MS analysis showed that the content of the residual cobalt in the crude product was $415.0 \mathrm{ppm}$, whereas $0.1 \mathrm{ppm}$ of Co was detected in the product obtained after passing through a pad of $\mathrm{Al}_{2} \mathrm{O}_{3}$.

Hydrosilylation of styrene with $\mathrm{Me}_{2} \mathrm{Si}(\mathrm{H}) \mathrm{O}\left(\mathrm{SiMe}_{2} \mathrm{O}\right)_{\mathrm{n}} \mathrm{Si}(\mathrm{H}) \mathrm{Me}_{2}$ by $\mathrm{Fe}(\mathrm{OPv})_{2} / \mathrm{CNAd}$ catalyst. (Table 3, entry 3 in the manuscript) In a $6 \mathrm{~mL}$ glass vial, $\mathrm{Fe}(\mathrm{OPv})_{2}(3 \mathrm{mg}, 0.01 \mathrm{mmol})$ and CNAd (3 mg, $\left.0.02 \mathrm{mmol}\right)$ were dissolved in DME $(0.1 \mathrm{~mL})$. Then $\mathrm{Me}_{2} \mathrm{Si}(\mathrm{H}) \mathrm{O}\left(\mathrm{SiMe}_{2} \mathrm{O}\right)_{\mathrm{n}} \mathrm{Si}(\mathrm{H}) \mathrm{Me}_{2}$ ( $\mathrm{n}=$ ca. 27, determined by $\left.{ }^{1} \mathrm{H} \mathrm{NMR}\right)$ $(1.07 \mathrm{~g}$, ca $1.0 \mathrm{mmol}$ for $\mathrm{Si}-\mathrm{H})$ and styrene $(149 \mu \mathrm{L}, 1.3 \mathrm{mmol})$ were added into the solution, and the resulting mixture was stirred at $80{ }^{\circ} \mathrm{C}$ for $24 \mathrm{~h}$. The mixture was cooled to room temperature, and the conversion of hydrosilane $(86 \%)$ and the yield of the product $(66 \%)$ were determined by ${ }^{1} \mathrm{H}-\mathrm{NMR}$ spectroscopy using anisole as the internal standard. By-products derived from the dehydrogenative hydrosilylation showing signals due to a $\mathrm{PhCH}=\mathrm{CH}-\mathrm{Si}$ group were concomitantly formed (the yield was estimated to be ca. $20 \%$ yield by the ${ }^{1} \mathrm{H}$ NMR). The resulting mixture was passed through a pad of $\mathrm{Al}_{2} \mathrm{O}_{3}$. The filtrate was dried under vacuum, then the product 25a containing the above described by-products was obtained in $81 \%$ yield $(948 \mathrm{mg})$.

Hydrosilylation of styrene with $\mathrm{Me}_{2} \mathrm{Si}(\mathrm{H}) \mathrm{O}\left(\mathrm{SiMe}_{2} \mathrm{O}\right)_{n} \mathrm{Si}(\mathrm{H}) \mathrm{Me}_{2}$ by $\mathrm{Fe}(\mathrm{OPv})_{2} / \mathrm{CNAd}$ catalyst in the presence of $(\mathbf{E t O})_{2} \mathrm{MeSiH}$ (Table 3, entry 4 in the manuscript). In a $6 \mathrm{~mL}$ glass vial, $\mathrm{Fe}(\mathrm{OPv})_{2}(3 \mathrm{mg}, 0.01$ mmol) and CNAd (3 mg, $0.02 \mathrm{mmol}),(\mathrm{EtO})_{2} \mathrm{MeSiH}(10 \mathrm{mg}, 0.08 \mathrm{mmol})$ were dissolved in DME $(0.1 \mathrm{~mL})$, then the mixture was stirred at room temperature for $1 \mathrm{~h}$. Then $\mathrm{Me}_{2} \mathrm{Si}(\mathrm{H}) \mathrm{O}\left(\mathrm{SiMe}_{2} \mathrm{O}\right)_{n} \mathrm{Si}(\mathrm{H}) \mathrm{Me}_{2}(\mathrm{n}=$ ca. 27 , determined by ${ }^{1} \mathrm{H}$ NMR) (1.07 g, ca $1.0 \mathrm{mmol}$ for $\left.\mathrm{Si}-\mathrm{H}\right)$, and styrene $(149 \mu \mathrm{L}, 1.3 \mathrm{mmol})$ were added into the solution, and the resulting mixture was stirred at $25{ }^{\circ} \mathrm{C}$ for $18 \mathrm{~h}$. The mixture was cooled to room temperature, 
and the conversion of hydrosilane (>99\%) and the yield of the product ( $>99 \%)$ were determined by ${ }^{1} \mathrm{H}-\mathrm{NMR}$ spectroscopy using anisole as the internal standard. In contrast to the reaction in the absence of the co-catalyst, no byproduct was formed. The resulting mixture was passed through a pad of $\mathrm{Al}_{2} \mathrm{O}_{3}$. The filtrate was dried under vacuum, then the product $\mathbf{2 5 a}$ was obtained in $96 \%$ yield $(1.13 \mathrm{~g})$.

${ }^{1} \mathrm{H}-\mathrm{NMR}\left(395 \mathrm{MHz}, \mathrm{CDCl}_{3}\right) \delta: 0.07$ (s, 139H, -SiMe $2_{2}$ ), 0.10(s, 18H, -SiMe $\left.2^{-}\right), 0.89-0.94\left(\mathrm{~m}, 4 \mathrm{H},-\mathrm{CH}_{2} \mathrm{Si}\right)$,

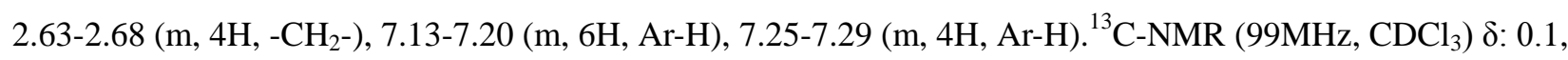
$1.0,1.1,20.2,29.3,125.5,127.8,128.3,145.1 .{ }^{29} \mathrm{Si}-\mathrm{NMR}\left(119 \mathrm{MHz}, \mathrm{CDCl}_{3}\right) \delta:-21.89,-21.28,7.09$.

ICP-MS: ICP-MS analysis showed that the content of the residual iron in the crude product was $416.2 \mathrm{ppm}$, whereas $2.5 \mathrm{ppm}$ of $\mathrm{Fe}$ was detected in the product obtained after passing through a pad of $\mathrm{Al}_{2} \mathrm{O}_{3}$.

\section{Hydrosilylation of vinylsiloxanes with hydrosiloxanes catalyzed by $\mathrm{Co}(\mathrm{OPv})_{2} / \mathrm{CNAd}$ catalysts (Table S15 and S16).}

In a $6 \mathrm{~mL}$ glass vial, $\mathrm{Co}(\mathrm{OPv})_{2}\{3 \mathrm{mg}(0.01 \mathrm{mmol}, 1 \mathrm{~mol} \%)$ or $8 \mathrm{mg}(0.03 \mathrm{mmol}, 3 \mathrm{~mol} \%)\}$ and CNAd (eq. to $\mathrm{Co}(\mathrm{OPv})_{2}$ charged) were dissolved in $\mathrm{DME}(0.10 \mathrm{~mL})$. Then the mixture of vinylsiloxane derivative (1 mmol) and hydrosiloxane $(1.3 \mathrm{mmol})$ was added. The resulting mixture was stirred at $80{ }^{\circ} \mathrm{C}$ for $24 \mathrm{~h}$. The mixture was cooled to room temperature, and the conversion of vinylsiloxane and the yield of the product were determined by ${ }^{1} \mathrm{H}-\mathrm{NMR}$ spectroscopy using anisole as the internal standard. The products were isolated by distillation under a reduced pressure or column chromatography.

Table S15. Hydrosilylation of vinylsiloxane with PMDS catalyzed by $\mathrm{Co}(\mathrm{OPv})_{2}$ with CNAd.

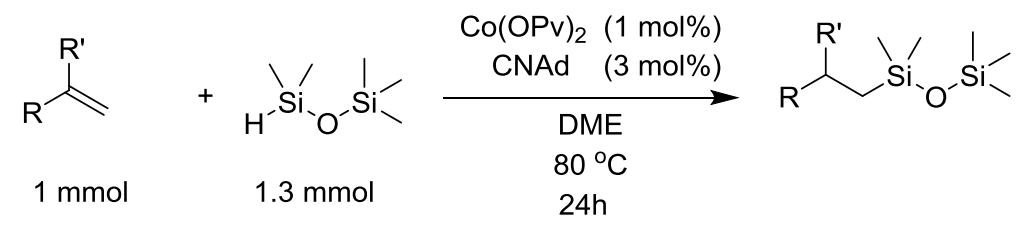

\begin{tabular}{|c|c|c|c|c|c|}
\hline Entry & Substrate & Product & Conv. $^{\mathrm{a}}(\%)$ & Selectivity $^{\mathrm{a}}(\%)$ & Isolated yield (\%) \\
\hline 1 & & $26 a$ & $>99$ & $>99$ & 79 \\
\hline 2 & 4 & $27 \mathbf{a}$ & $>99$ & $>99$ & 97 \\
\hline
\end{tabular}

${ }^{\text {a }}$ Conversion and the selectivity of the product were determined by both ${ }^{1} \mathrm{H}$ NMR and GC spectra of the crude products.

\section{1,2-Bis(1,1,1-3,3-pentamethyldisiloxanyl)ethane (26a)}

$\mu \mathrm{L}, 1.3 \mathrm{mmol})$ for $24 \mathrm{~h}$ at $80{ }^{\circ} \mathrm{C}$ catalyzed by $\mathrm{Co}(\mathrm{OPv})_{2}(3 \mathrm{mg}, 0.01 \mathrm{mmol})$ and CNAd (5 mg, $0.03 \mathrm{mmol})$ in DME $(0.10 \mathrm{~mL}$ ) (Table $\mathrm{S} 15$, entry 1$)$. The product was purified by passing through the alumina column. Isolated yield: $255 \mathrm{mg}(79 \%) .{ }^{1} \mathrm{H}-\mathrm{NMR}\left(395 \mathrm{MHz}, \mathrm{CDCl}_{3}\right) \delta: 0.03\left(\mathrm{~s}, 12 \mathrm{H},-\mathrm{SiMe}_{2}-\right), 0.06$ $\left(\mathrm{s}, 18 \mathrm{H},-\mathrm{SiMe}_{3}\right), 0.40$ (s, 4H, - $\mathrm{CH}_{2}$ ). ${ }^{13} \mathrm{C}-\mathrm{NMR}\left(99 \mathrm{MHz}, \mathrm{CDCl}_{3}\right) \delta:-0.4,2.0,9.6 .{ }^{29} \mathrm{Si}-\mathrm{NMR}(119 \mathrm{MHz}$, $\left.\mathrm{CDCl}_{3}\right) \delta: 7.02,8.44$. HRMS (EI) calcd for $\left[\mathrm{C}_{12} \mathrm{H}_{34} \mathrm{O}_{2} \mathrm{Si}_{4}\right]: 322.1636$. found 322.1636. 


\section{1,1,1,3,5,5,5-heptamethyl-3-(2-(1,1,3,3,3-pentamethyldisiloxanyl)ethyl)trisiloxane (27a)}

The product 27a as obtained from the reaction of

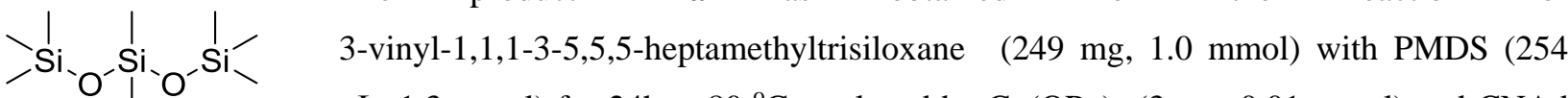
$\mu \mathrm{L}, 1.3 \mathrm{mmol})$ for $24 \mathrm{~h}$ at $80{ }^{\circ} \mathrm{C}$ catalyzed by $\mathrm{Co}(\mathrm{OPv})_{2}(3 \mathrm{mg}, 0.01 \mathrm{mmol})$ and CNAd $(5 \mathrm{mg}, 0.03 \mathrm{mmol})$ in DME $(0.10 \mathrm{~mL}$ ) (Table $\mathrm{S} 15$, entry 2). The product was isolated by distillation $\left(8\right.$ torr, $110{ }^{\circ} \mathrm{C}$ ). Isolated yield : $387 \mathrm{mg}(97 \%) .{ }^{1} \mathrm{H}-\mathrm{NMR}(395 \mathrm{MHz}$, $\left.\mathrm{CDCl}_{3}\right) \delta: 0.00$ (s, 3H, -SiMe-), 0.03 (s, 6H, -SiMe $\left.2^{-}\right), 0.06$ (s, 9H, -SiMe 3 ), 0.09 (s, 18H, - $-\mathrm{SiMe}_{3}$ ), 0.31-0.44 $\left(\mathrm{m}, 4 \mathrm{H},-\mathrm{CH}_{2} \mathrm{Si}\right) .{ }^{13} \mathrm{C}-\mathrm{NMR}\left(99 \mathrm{MHz}, \mathrm{CDCl}_{3}\right) \delta:-1.2,-0.4,1.8,2.0,8.9,9.5 .{ }^{29} \mathrm{Si}-\mathrm{NMR}\left(119 \mathrm{MHz}, \mathrm{CDCl}_{3}\right) \delta$ : -20.70, 6.90, 7.05, 8.52. HRMS (EI) calcd for $\left[\mathrm{C}_{14} \mathrm{H}_{40} \mathrm{O}_{3} \mathrm{Si}_{5}\right]$ : 396.1824. found 396.1823.

Table S16. Hydrosilylation of vinylsiloxane with MD'M catalyzed by $\mathrm{Co}(\mathrm{OPv})_{2}$ with CNAd.

\begin{tabular}{|c|c|c|c|c|c|c|}
\hline & Imol & 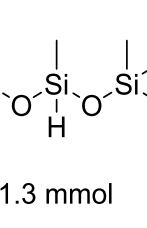 & $\begin{array}{r}\mathrm{Co}(\mathrm{OPV})_{2} \\
\mathrm{CNAd} \quad(3 \\
\mathrm{DME} \\
80^{\circ} \mathrm{G} \\
24 \mathrm{~h}\end{array}$ & $\begin{array}{l}\mathrm{mol} \%) \\
\mathrm{mol} \%)\end{array}$ & $\mid \mathrm{Si}_{1}, \mathrm{Si}_{\mathbf{2}}$ & \\
\hline Entry & Substrate & Product & $\begin{array}{c}\text { Catalyst } \\
\text { loading } \\
\text { (x mol\%) }\end{array}$ & $\begin{array}{c}\text { Conv. }^{a} \\
(\%)\end{array}$ & $\begin{array}{c}\text { Selectivity }^{\mathrm{a}} \\
(\%)\end{array}$ & $\begin{array}{c}\text { Isolated } \\
\text { yield }(\%)\end{array}$ \\
\hline 1 & & $27 \mathbf{a}$ & 3 & $>99$ & $>99$ & 84 \\
\hline 2 & & $27 a$ & 1 & 35 & $>99$ & - \\
\hline 3 & sis. & $28 a$ & 3 & $>99$ & $>99$ & 80 \\
\hline 4 & & $28 \mathrm{a}$ & 1 & 11 & $>99$ & - \\
\hline
\end{tabular}

${ }^{\text {a }}$ Conversion and the selectivity of the product were determined by both ${ }^{1} \mathrm{H}$ NMR and GC spectra of the crude products.

\section{1,1,1,3,5,5,5-heptamethyl-3-(2-(1,1,3,3,3-pentamethyldisiloxanyl)ethyl)trisiloxane (27a)}

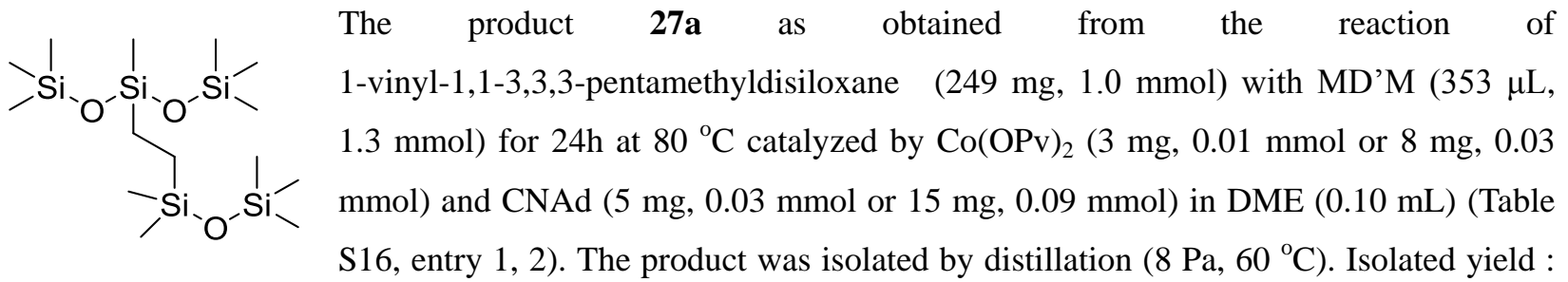
$334 \mathrm{mg}(84 \%) .{ }^{1} \mathrm{H}-\mathrm{NMR}\left(395 \mathrm{MHz}, \mathrm{CDCl}_{3}\right) \delta: 0.00$ (s, 3H, -SiMe-), 0.03 (s, 6H, -SiMe $2^{-}$), 0.06 (s, 9H, - $\mathrm{SiMe}_{3}$ ), 0.09 (s, 18H, - $\mathrm{SiMe}_{3}$ ), 0.31-0.44 (m, 4H, $\left.-\mathrm{CH}_{2} \mathrm{Si}\right) .{ }^{13} \mathrm{C}-\mathrm{NMR}\left(99 \mathrm{MHz}, \mathrm{CDCl}_{3}\right) \delta$ : -1.2, -0.4, 1.8, 2.0, 8.9, 9.5. ${ }^{29} \mathrm{Si}-\mathrm{NMR}\left(119 \mathrm{MHz}, \mathrm{CDCl}_{3}\right) \delta:-20.70,6.90,7.05,8.52$. HRMS (EI) calcd for $\left[\mathrm{C}_{14} \mathrm{H}_{40} \mathrm{O}_{3} \mathrm{Si}_{5}\right]: 396.1824$. found 396.1823. 


\section{1,2-Bis(1,1,1-3-5,5,5-heptamethyltrisiloxanyl)ethane (28a)}<smiles>C[Si](C)(C)O[Si](C)(CC[Si](C)(O[Si](C)(C)C)O[Si](C)(C)C)O[Si](C)(C)C</smiles>

The product 28 a

3-vinyl-1,1,1-3-5,5,5-heptamethyltrisiloxane (249 mg $1.0 \mathrm{mmol})$ with MD’M (353 $\mu \mathrm{L} 1.3 \mathrm{mmol})$ for $24 \mathrm{~h}$ at $80{ }^{\circ} \mathrm{C}$ catalyzed by $\mathrm{Co}(\mathrm{OPv})_{2}(3 \mathrm{mg}, 0.01 \mathrm{mmol}$ or $8 \mathrm{mg}, 0.03$ mmol) and CNAd (5 mg, $0.03 \mathrm{mmol}$ or $15 \mathrm{mg}, 0.09 \mathrm{mmol})$ in DME $(0.10 \mathrm{~mL})$ (Table S16, entry 3,4$)$. The product was isolated by distillation $\left(10 \mathrm{~Pa}, 80{ }^{\circ} \mathrm{C}\right)$. Isolated yield: 382mg (80\%). ${ }^{1} \mathrm{H}-\mathrm{NMR}\left(395 \mathrm{MHz}, \mathrm{CDCl}_{3}\right) \delta: 0.00$ (s, 6H, -SiMe-), 0.08 (s, 36H, -SiMe 3 ), 0.35 (s, 4H, - $\mathrm{CH}_{2} \mathrm{Si}$ ). ${ }^{13} \mathrm{C}-\mathrm{NMR}\left(99 \mathrm{MHz}, \mathrm{CDCl}_{3}\right) \delta:-1.2,1.8,8.8 .{ }^{29} \mathrm{Si}-\mathrm{NMR}\left(119 \mathrm{MHz}, \mathrm{CDCl}_{3}\right) \delta:-20.66,6.90$. HRMS (EI) calcd for $\left[\mathrm{C}_{16} \mathrm{H}_{46} \mathrm{O}_{4} \mathrm{Si}_{6}\right]:$ 470.2012. found 470.2011.

\section{Cross-linking of siloxane polymers: a preliminary study by the $\mathrm{Co}(\mathrm{OPv})_{2}$ with CNAd catalyst.}

In a $6 \mathrm{~mL}$ glass vial were placed $\mathrm{Co}(\mathrm{OPv})_{2}(3 \mathrm{mg}, 0.01 \mathrm{mmol})$ and CNAd $(3 \mathrm{mg}, 0.03 \mathrm{mmol})$, and a mixture of $\mathrm{CH}_{2}=\mathrm{CHSiMe}_{2} \mathrm{O}\left(\mathrm{SiMe}_{2} \mathrm{O}\right)_{\mathrm{n}} \mathrm{SiMe}_{2} \mathrm{CH}=\mathrm{CH}_{2}$ ( $\mathrm{n}=\mathrm{ca} .47$, determined by ${ }^{1} \mathrm{H}-\mathrm{NMR}$ and $\left.{ }^{29} \mathrm{Si}-\mathrm{NMR}\right)(2.87 \mathrm{~g}$, ca 1.56 mmol for $\left.\mathrm{Si}-\mathrm{CH}=\mathrm{CH}_{2}\right)$ and $\mathrm{Me}_{3} \mathrm{SiO}[\mathrm{Si}(\mathrm{H}) \mathrm{MeO}]_{\mathrm{m}} \mathrm{SiMe}_{3}\left(\mathrm{~m}=\right.$ ca. 8 , determined by ${ }^{1} \mathrm{H}-\mathrm{NMR}$ and $\left.{ }^{29} \mathrm{Si}-\mathrm{NMR}\right)$ $(0.13 \mathrm{~g}$, ca $1.62 \mathrm{mmol}$ for $\mathrm{Si}-\mathrm{H})$ was added. The resulting mixture was stirred at $120{ }^{\circ} \mathrm{C}$ under nitrogen atmosphere. The solution set to gel after $15 \mathrm{~min}$. And then the resulting mixture was kept at $120{ }^{\circ} \mathrm{C}$ for $0.5 \mathrm{~h}, 1$ h, $3 \mathrm{~h}$, or $24 \mathrm{~h}$. Figure $\mathrm{S} 1$ shows the photographs of the $\mathrm{Co}(\mathrm{OPv})_{2} / \mathrm{CNAd}$ system to produce the cross-linked siloxane polymer.
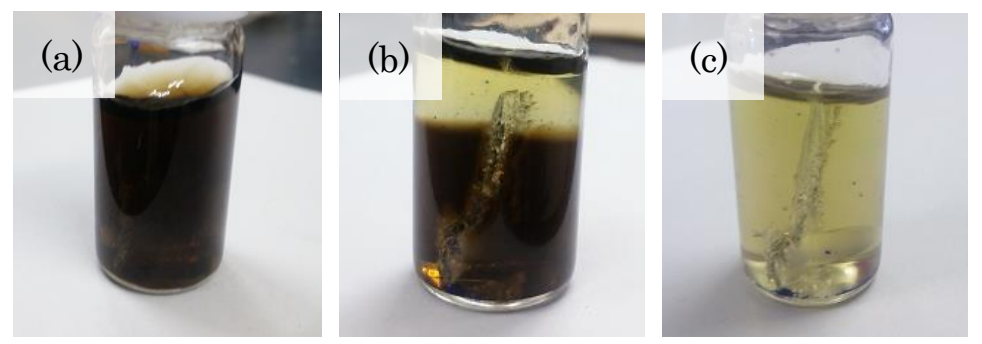

(d)

Figure S1. Photographs of the $\mathrm{Co}(\mathrm{OPv})_{2} / \mathrm{CNAd}$ system to produce the cross-linked siloxane polymer; (a) after the reaction at $120^{\circ} \mathrm{C}$ for $3 \mathrm{~h}$, (b) the formed siloxane polymer exposed to air for $3 \mathrm{~h}$, (c) the formed siloxane polymer exposed to air for $12 \mathrm{~h},(\mathrm{~d})$ cross-linked silicone polymer.

Cross-linking of siloxane polymers using Karstedt's catalyst. In a $6 \mathrm{~mL}$ glass vial were placed Karstedt's catalyst in xylene $(2 \mathrm{wt} \% / \mathrm{Pt}) \quad(3.9 \quad \mathrm{mg}, \quad 0.078 \quad \mathrm{mg} / \mathrm{Pt}, \quad c a \quad 26 \quad \mathrm{ppm} / \mathrm{Pt}), \quad$ and $\mathrm{CH}_{2}=\mathrm{CHSiMe}{ }_{2} \mathrm{O}\left(\mathrm{SiMe}_{2} \mathrm{O}\right){ }_{n} \mathrm{SiMe}_{2} \mathrm{CH}=\mathrm{CH}_{2}$ ( $\mathrm{n}=$ ca. 47 , determined by ${ }^{1} \mathrm{H}-\mathrm{NMR}$ and $\left.{ }^{29} \mathrm{Si}-\mathrm{NMR}\right)(2.87 \mathrm{~g}$, ca 1.56 mmol for $\mathrm{Si}-\mathrm{CH}=\mathrm{CH}_{2}$ ). Then, $\mathrm{Me}_{3} \mathrm{SiO}[\mathrm{Si}(\mathrm{H}) \mathrm{MeO}]_{\mathrm{m}} \mathrm{SiMe}_{3}\left(\mathrm{~m}=\mathrm{ca} .8\right.$, determined by ${ }^{1} \mathrm{H}-\mathrm{NMR}$ and $\left.{ }^{29} \mathrm{Si}-\mathrm{NMR}\right)$ $(0.13 \mathrm{~g}$, ca $1.62 \mathrm{mmol}$ for $\mathrm{Si}-\mathrm{H})$ was added into the mixture. The resulting mixture was stirred at room temperature. The solution set to gel after 1 minute. The resulting solid was kept at room temperature for several hours. The colorless solid was obtained, and this cross-linked siloxane polymer was analyzed by ${ }^{1} \mathrm{H}$ quantitative NMR (Fig. S2(b)), ${ }^{29}$ Si DD NMR (Fig. S3(b)), and FT-IR (Fig. S4, light blue line) spectroscopy. 


\section{Characterization of the silicone gel formed.}

The cross-linked silicone gel was characterized by ${ }^{1} \mathrm{H}$ quantitative NMR (Fig. S2), ${ }^{29} \mathrm{Si}$ DD NMR (Fig. S3), and FT-IR (Fig. S4) spectroscopies. Spectral data of the gel prepared by the $\mathrm{Co}(\mathrm{OPv})_{2}$ catalyst were compared with those of the gel prepared by Karstedt's catalyst.

\section{Characterization by ${ }^{1} \mathrm{H}$ quantitative NMR.}

${ }^{1} \mathrm{H} \quad \mathrm{NMR}$ spectrum of a $1: 1$ mixture of $\mathrm{Me}_{3} \mathrm{SiO}[\mathrm{Si}(\mathrm{H}) \mathrm{MeO}]_{\mathrm{m}} \mathrm{SiMe}_{3} \quad(\mathrm{~m}=$ ca. 8$)$, and $\mathrm{CH}_{2}=\mathrm{CHSiMe}_{2} \mathrm{O}\left(\mathrm{SiMe}_{2} \mathrm{O}\right)_{\mathrm{n}} \mathrm{SiMe}_{2} \mathrm{CH}=\mathrm{CH}_{2}(\mathrm{n}=$ ca. 47$)$, in $\mathrm{CDCl}_{3}$ was shown in Figure S2-(a). The methyl groups of two siloxanes appeared at around $0 \mathrm{ppm}$, the hydrogen of the $\mathrm{Si}-\mathrm{H}$ group of $\mathrm{Me}_{3} \mathrm{SiO}[\mathrm{Si}(\mathrm{H}) \mathrm{MeO}]_{\mathrm{m}} \mathrm{SiMe}_{3}$ was observed at $4.8 \mathrm{ppm}$, and the vinyl group of $\mathrm{CH}_{2}=\mathrm{CHSiMe}_{2} \mathrm{O}\left(\mathrm{SiMe}_{2} \mathrm{O}\right)_{\mathrm{n}} \mathrm{SiMe}_{2} \mathrm{CH}=\mathrm{CH}_{2}$ appeared at between $5.5 \mathrm{ppm}$ and $6.3 \mathrm{ppm}$.

The ${ }^{1} \mathrm{H}$ solid state quantitative NMR spectrum of the cross-linked siloxane polymer obtained by the reaction mediated by Karstedt's catalyst was shown in Figure S2-(b). The vinyl protons at around $4.5 \sim 6.5 \mathrm{ppm}$ almost disappeared because the cross-linking reaction of vinyl siloxane and hydrosiloxane occurred quantitatively.

The ${ }^{1} \mathrm{H}$ solid state quantitative NMR spectra of the cross-linked siloxane polymer obtained by the reaction catalyzed by $\mathrm{Co}(\mathrm{OPv})_{2} / \mathrm{CNAd}$ catalyst system at $120{ }^{\circ} \mathrm{C}$ after $0.5 \mathrm{~h}, 1 \mathrm{~h}, 3 \mathrm{~h}$ and $24 \mathrm{~h}$ were shown in Figure S2-(c), (d), (e) and (f), respectively. In each spectrum, integral ratios of signals due to the $\mathrm{CH}_{2}=\mathrm{CHSiMe}_{2}$ and the $\mathrm{H}-\mathrm{SiMe}$ groups relative to that of $\mathrm{SiMe}_{2}$ moiety were estimated to be (c) $54 \%$ and $55 \%$, (d) $53 \%$ and $47 \%$, (e) $75 \%$ and $68 \%$, and (f) $76 \%$ and $68 \%$, respectively, which indicate the progress of cross-linking reaction.

\section{Characterization by ${ }^{29} \mathrm{Si} D \mathrm{DD}$ NMR.}

Figure S3-(a) showed the ${ }^{29} \mathrm{Si}$ quantitative NMR spectrum of a 1:1 mixture of $\mathrm{Me}_{3} \mathrm{SiO}(\mathrm{Si}(\mathrm{H}) \mathrm{MeO})_{\mathrm{m}} \mathrm{SiMe}_{3}$ ( $\mathrm{m}=$ ca. 8) and $\mathrm{CH}_{2}=\mathrm{CHSiMe}_{2} \mathrm{O}\left(\mathrm{SiMe}_{2} \mathrm{O}\right)_{\mathrm{n}} \mathrm{SiMe}_{2} \mathrm{CH}=\mathrm{CH}_{2}(\mathrm{n}=$ ca. 47$)$ in $\mathrm{CDCl}_{3}$. The ${ }^{29} \mathrm{Si}$ resonance at -3.8 ppm was assignable to the $\mathrm{HSiMe}$ unit, whereas that of $\mathrm{SiMe}_{2}$ and $\mathrm{CH}_{2}=\mathrm{CHSiMe}_{2}$ moieties appeared at around $-21 \mathrm{ppm}$. The ${ }^{29} \mathrm{Si}$ solid state quantitative NMR spectrum of the cross-linked siloxane polymer obtained by the reaction mediated by Karstedt's catalyst was shown in Figure S3-(b). The peak at -3.8 ppm disappeared because the cross-linking reaction occurred quantitatively. In contrast, those of the cross-linked siloxane polymer obtained by the reaction catalyzed by $\mathrm{Co}(\mathrm{OPv})_{2} / \mathrm{CNAd}$ catalyst system at $120{ }^{\circ} \mathrm{C}$ after $0.5,1 \mathrm{~h}, 3$ and 24h shown in Figure S3-(c), (d), (e) and (f), respectively, indicate the progress of cross-linking as (c) 58\%, (d) $56 \%$, (e) $66 \%$, and (f) $71 \%$, respectively.

\section{Characterization by IR spectroscopy.}

The orange line in Figure S4 shows the FT-IR spectrum of a 1:1 mixture of $\mathrm{Me}_{3} \mathrm{SiO}(\mathrm{Si}(\mathrm{H}) \mathrm{MeO})_{\mathrm{m}} \mathrm{SiMe}_{3}(\mathrm{~m}=$ ca. 8) and $\mathrm{CH}_{2}=\mathrm{CHSiMe}_{2} \mathrm{O}\left(\mathrm{SiMe}_{2} \mathrm{O}\right)_{n} \mathrm{SiMe}_{2} \mathrm{CH}=\mathrm{CH}_{2}$ ( $\mathrm{n}=$ ca. 47). The peaks derived from the $\mathrm{Si}-\mathrm{H}$ bond vibration of the hydrosiloxane appeared at $2150 \mathrm{~cm}^{-1}$, whereas the $\mathrm{C}=\mathrm{C}$ double bond vibration of the vinylsiloxane was observed at $1423 \mathrm{~cm}^{-1}$. The light blue line in Figure S4 shows the FT-IR spectrum of the cross-linked siloxane polymer obtained by the reaction mediated by the Karstedt's catalyst. The peak at 2150 and $1423 \mathrm{~cm}^{-1}$ completely disappeared because quantitative cross-linking took place. The spectra of the siloxane polymer obtained by the 
$\mathrm{Co}(\mathrm{OPv})_{2} / \mathrm{CNAd}$ catalysis at $120{ }^{\circ} \mathrm{C}$ for $0.5 \mathrm{~h}, 1 \mathrm{~h}, 3 \mathrm{~h}$ and $24 \mathrm{~h}$ were shown as the brown, green, violet and blue colored lines, respectively, in Figure S4, suggesting cross-linking was approximately half. 


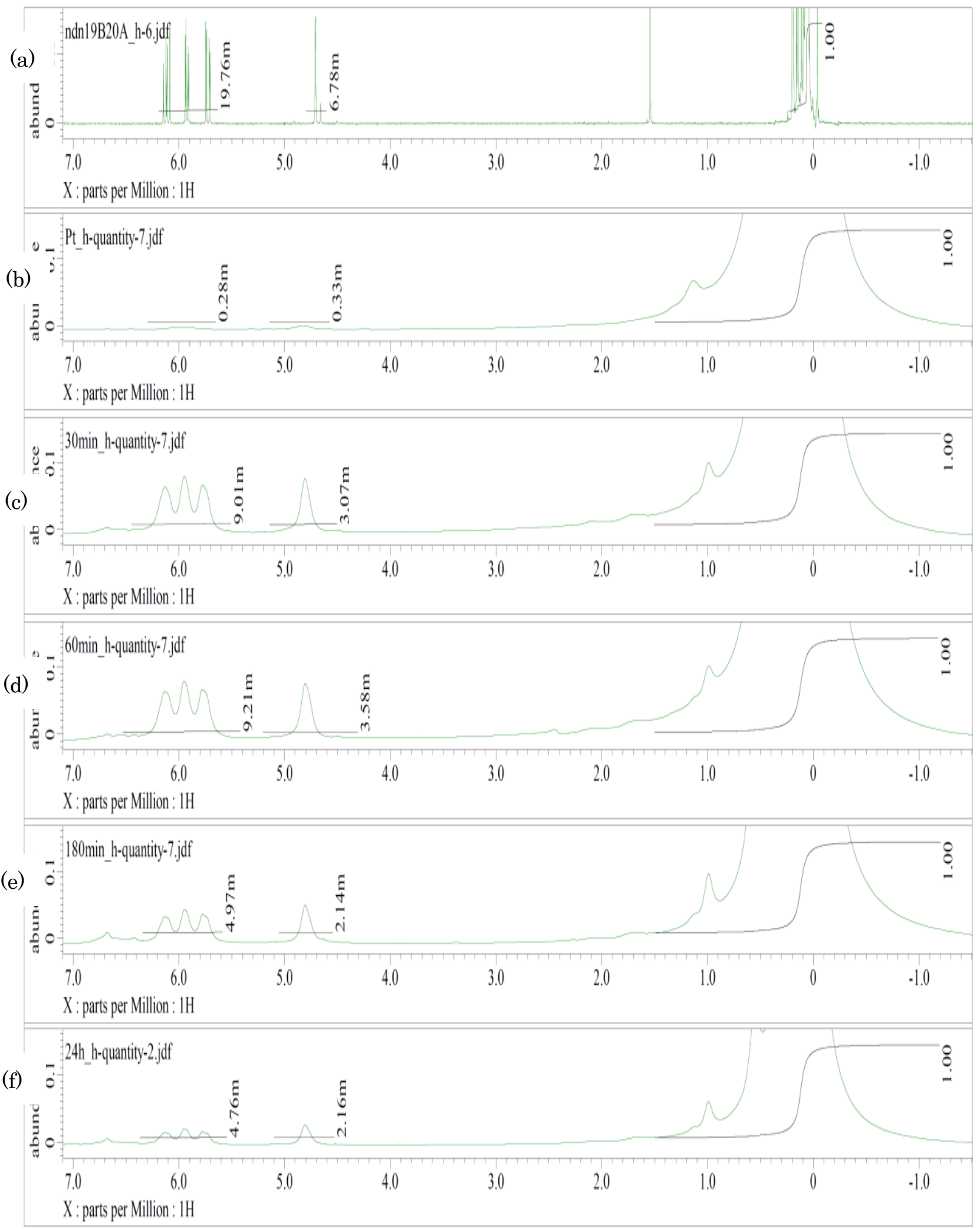

Figure S2. ${ }^{1} \mathrm{H}$ NMR spectrum of (a) the mixture of a $1: 1$ mixture of $\mathrm{Me}_{3} \mathrm{SiO}(\mathrm{Si}(\mathrm{H}) \mathrm{MeO})_{\mathrm{m}} \mathrm{SiMe}_{3}(\mathrm{~m}=\mathrm{ca} .8)$ and $\mathrm{CH}_{2}=\mathrm{CHSiMe} \mathrm{O}_{2} \mathrm{O}\left(\mathrm{SiMe}_{2} \mathrm{O}\right)_{n} \mathrm{SiMe}_{2} \mathrm{CH}=\mathrm{CH}_{2}(\mathrm{n}=$ ca. 47$)$ in $\mathrm{CDCl}_{3}$ without catalyst. ${ }^{1} \mathrm{H}$ quantitative NMR spectra of cross-linked silicones obtained by the reaction catalyzed by (b) Karstedt's catalyst $(26 \mathrm{ppm} / \mathrm{Pt})$ at room temperature, or $\mathrm{Co}(\mathrm{OPv})_{2}(195 \mathrm{ppm} / \mathrm{Co})$ with CNAd at $120^{\circ} \mathrm{C}$ for (c) $0.5 \mathrm{~h}$, (d) $1 \mathrm{~h}$, (e) $3 \mathrm{~h}$, and (f) $24 \mathrm{~h}$. 


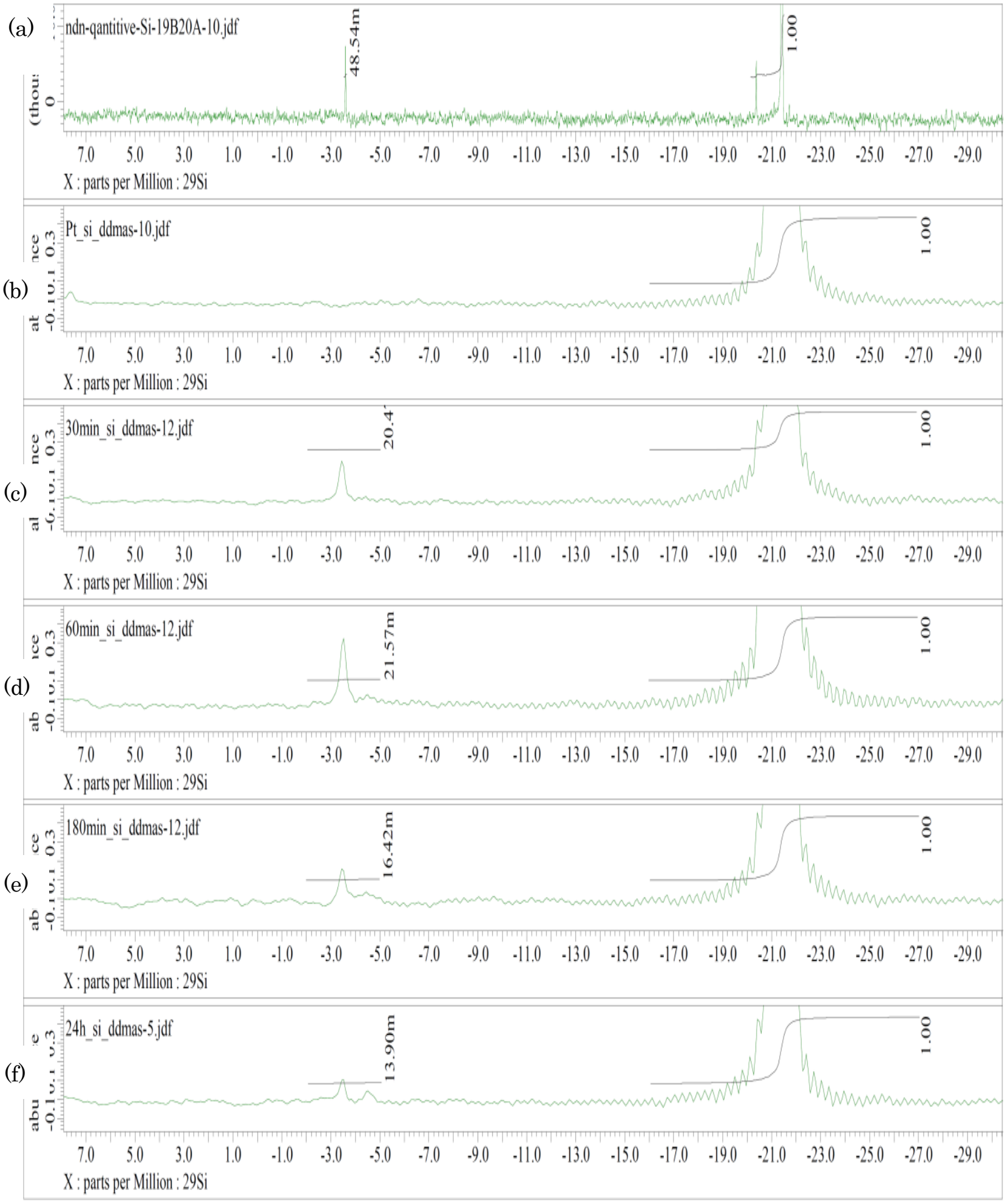

Figure S3. ${ }^{29} \mathrm{Si}$ quantitative NMR spectrum of (a) the mixture of a $1: 1$ mixture of $\mathrm{Me}_{3} \mathrm{SiO}(\mathrm{Si}(\mathrm{H}) \mathrm{MeO})_{\mathrm{m}} \mathrm{SiMe}_{3}$ ( $\mathrm{m}=$ ca. 8) and $\mathrm{CH}_{2}=\mathrm{CHSiMe}_{2} \mathrm{O}\left(\mathrm{SiMe}_{2} \mathrm{O}\right)_{n} \mathrm{SiMe}_{2} \mathrm{CH}=\mathrm{CH}_{2}(\mathrm{n}=$ ca. 47$)$ in $\mathrm{CDCl}_{3}$ without catalyst. ${ }^{29} \mathrm{Si} \mathrm{DD}$ NMR spectra of cross-linked silicones obtained by the reaction catalyzed by (b) Karstedt's catalyst ( $26 \mathrm{ppm} / \mathrm{Pt}$ ) at room temperature, or $\mathrm{Co}(\mathrm{OPv})_{2}(195 \mathrm{ppm} / \mathrm{Co})$ with $\mathrm{CNAd}$ at $120{ }^{\circ} \mathrm{C}$ for (c) $0.5 \mathrm{~h}$, (d) $1 \mathrm{~h}$, (e) $3 \mathrm{~h}$, and (f) $24 \mathrm{~h}$. 


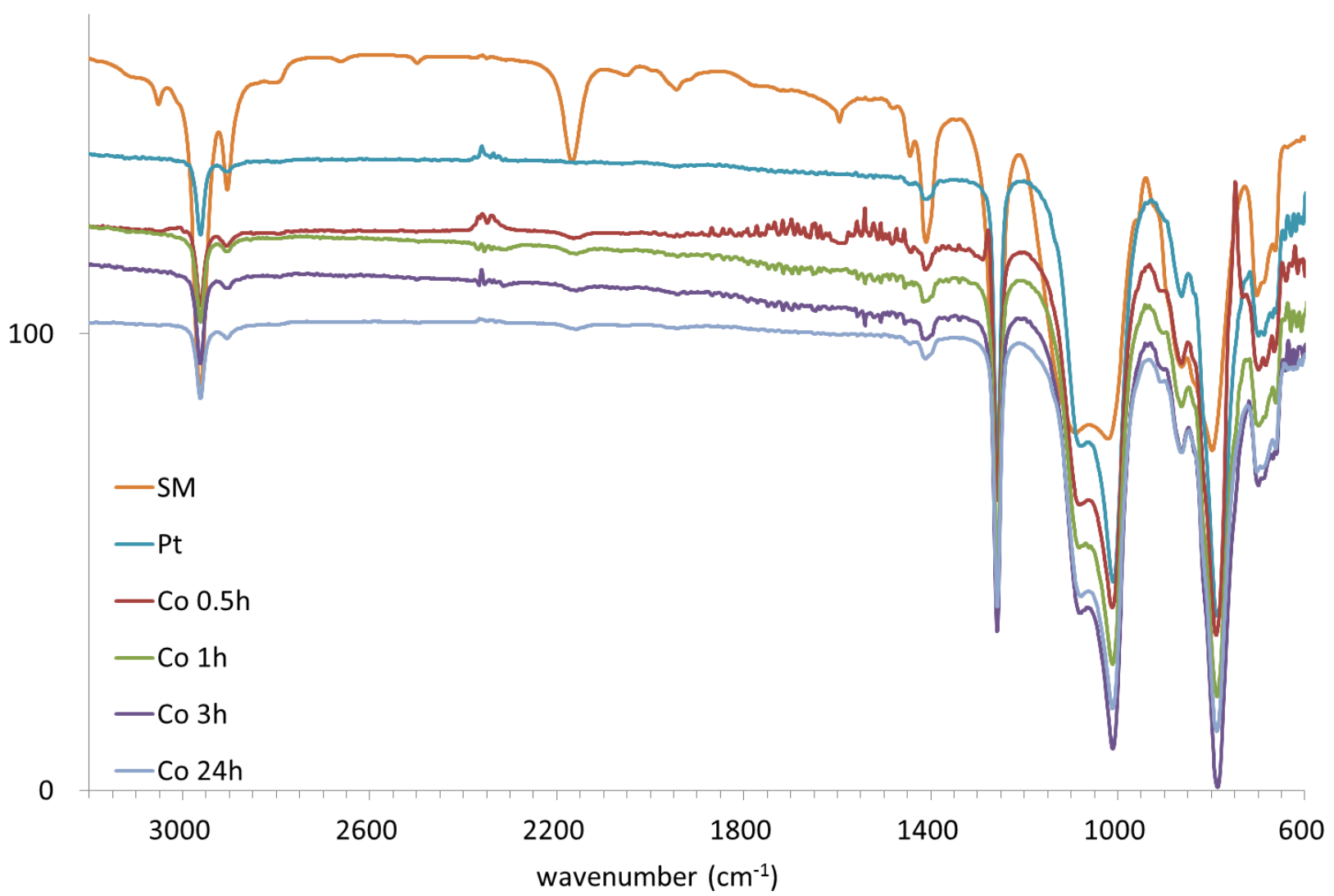

Figure S4. FT-IR spectra of (a) a $1: 1$ mixture of $\mathrm{Me}_{3} \mathrm{SiO}(\mathrm{Si}(\mathrm{H}) \mathrm{MeO})_{\mathrm{m}} \mathrm{SiMe}_{3}(\mathrm{~m}=$ ca. 8) and $\mathrm{CH}_{2}=\mathrm{CHSiMe}_{2} \mathrm{O}\left(\mathrm{SiMe}_{2} \mathrm{O}\right)_{\mathrm{n}} \mathrm{SiMe}_{2} \mathrm{CH}=\mathrm{CH}_{2}(\mathrm{n}=\mathrm{ca}$. 47). Cross-linked silicones obtained by the reaction catalyzed by (b) Karstedt's catalyst (26 ppm/Pt) at room temperature, or $\mathrm{Co}(\mathrm{OPv})_{2}(195 \mathrm{ppm} / \mathrm{Co})$ with CNAd at $120{ }^{\circ} \mathrm{C}$ for (c) $0.5 \mathrm{~h},(\mathrm{~d}) 1 \mathrm{~h}$, (e) $3 \mathrm{~h}$, and (f) $24 \mathrm{~h}$. 


\section{Catalytic activity of air-exposed $\mathrm{M}(\mathrm{OPv})_{2}$ for the hydrosilylation.}

Hydrosilylation of styrene with PMDS by the air-exposed $\mathrm{Fe}(\mathrm{OPv})_{2} / \mathrm{CNAd}$ catalyst. Prior to the hydrosilylation experiment, $\mathrm{Fe}(\mathrm{OPv})_{2}$ was exposure to air at room temperature for 3 days. The color of $\mathrm{Fe}(\mathrm{OPv})_{2}$ was changed from pale green to brown. In a $6 \mathrm{~mL}$ glass vial, the air-exposed $\mathrm{Fe}(\mathrm{OPv})_{2}(3 \mathrm{mg} 0.01 \mathrm{mmol})$ and CNAd (3 mg, $0.02 \mathrm{mmol})$ were dissolved in DME $(0.10 \mathrm{~mL})$. The mixture was stirred at room temperature for $1 \mathrm{hr}$ under nitrogen atmosphere, followed by the addition of mixture of styrene $(115 \mu \mathrm{L}, 1.0 \mathrm{mmol})$ and PMDS $(254 \mu \mathrm{L} 1.3$ mmol). The resulting mixture was stirred at $50{ }^{\circ} \mathrm{C}$. Relatively long induction period (several hours) was observed. After $24 \mathrm{~h}$, the conversion of styrene and the yield of the product 1a, which were determined by ${ }^{1}$ H-NMR spectroscopy using anisole as the internal standard, reached $50 \%$. The induction period disappeared when $(\mathrm{EtO})_{2} \mathrm{MeSiH}$ was used as the co-catalyst. In a $6 \mathrm{~mL}$ glass vial, the $\mathrm{Fe}(\mathrm{OPv})_{2}(3 \mathrm{mg} 0.01 \mathrm{mmol})$ and CNAd (3 mg, $0.02 \mathrm{mmol})$ were dissolved in DME $(0.10 \mathrm{~mL})$, then $(\mathrm{EtO})_{2} \mathrm{MeSiH}(5 \mathrm{mg}, 0.04 \mathrm{mmol})$ was added into the solution. The mixture was stirred at room temperature for $1.5 \mathrm{hr}$ under nitrogen atmosphere, followed by the addition of the mixture of styrene $(115 \mu \mathrm{L}, 1.0 \mathrm{mmol})$ and PMDS (254 $\mu \mathrm{L} 1.3 \mathrm{mmol})$. The resulting mixture was stirred at $50{ }^{\circ} \mathrm{C}$ for $3 \mathrm{~h}$. Both the conversion of styrene and the yield of $\mathbf{1 a}$ were $>99 \%$.

Hydrosilylation of $\alpha$-methylstyrene with PMDS by the air-exposed $\mathrm{Co}(\mathrm{OPv})_{2} / \mathrm{CNAd}$ catalyst. Prior to the hydrosilylation experiment, $\mathrm{Co}(\mathrm{OPv})_{2}$ was exposure to air at room temperature for 3 days. No significant color change of $\mathrm{Co}(\mathrm{OPv})_{2}$ was observed. In a $6 \mathrm{~mL}$ glass vial, the $\mathrm{Co}(\mathrm{OPv})_{2}(3 \mathrm{mg} 0.01 \mathrm{mmol})$ and CNAd (3 mg, $0.02 \mathrm{mmol})$ were dissolved in DME $(0.10 \mathrm{~mL})$. The mixture was stirred at room temperature for $1 \mathrm{hr}$ under nitrogen atmosphere, followed by the addition of the mixture of $\alpha$-methylstyrene (130 $\mu \mathrm{L}, 1.0 \mathrm{mmol})$ and PMDS ( $254 \mu \mathrm{L} 1.3 \mathrm{mmol})$. The resulting mixture was stirred at $80{ }^{\circ} \mathrm{C}$ for $24 \mathrm{~h}$. Induction period was observed. After $24 \mathrm{~h}$, the conversion of $\alpha$-methylstyrene and the yield of the product $\mathbf{2 a}$, which were determined by

${ }^{1} \mathrm{H}-\mathrm{NMR}$ spectroscopy using anisole as the internal standard, reached $80 \%$. Use of $(\mathrm{EtO})_{3} \mathrm{SiH}$ as the co-catalyst contributed to the rate acceleration. In a $6 \mathrm{~mL}$ glass vial, the $\mathrm{Co}(\mathrm{OPv})_{2}(3 \mathrm{mg} 0.01 \mathrm{mmol})$ and CNAd (3 $\mathrm{mg}$, $0.02 \mathrm{mmol})$ were dissolved in $\mathrm{DME}(0.10 \mathrm{~mL})$, then $(\mathrm{EtO})_{3} \mathrm{SiH}(7 \mathrm{mg}, 0.04 \mathrm{mmol})$ was added into the solution. The mixture was stirred at room temperature for $1.5 \mathrm{hr}$ under nitrogen atmosphere, followed by the addition of the mixture of $\alpha$-methylstyrene ( $130 \mu \mathrm{L}, 1.0 \mathrm{mmol}$ ) and PMDS (254 $\mu \mathrm{L} 1.3 \mathrm{mmol})$. The resulting mixture was stirred at $50{ }^{\circ} \mathrm{C}$ for $3 \mathrm{~h}$. The resulting mixture was stirred at $50{ }^{\circ} \mathrm{C}$ for $3 \mathrm{~h}$. Both the conversion of $\alpha$-methyl styrene and the yield of $\mathbf{2 a}$ were $>99 \%$.

Comments on the mechanistic aspects and functional group compatibility. During the reviewing process, one of the reviewers requested to give our comments on the following issues. These should be awaited for further detailed studies; however, it is worthwhile to give short comments based on the preliminary experiments.

\section{Comments to the $\mathrm{Hg}$ test}

In the platinum-catalyzed hydrosilylations, possible involvement of platinum colloids was proposed. Addition of mercury (Hg test) to make sure no significant retardation of the reaction rate was often performed to exclude the involvement of nanoparticles in catalysis. We performed the Hg test as follows: hydrosilylation of styrene with 1,1,3,3,3-pentamethyldisiloxane (PMDS) catalyzed by $\mathrm{Fe}(\mathrm{OPv})_{2}(1 \mathrm{~mol} \%)$ 
and CNAd ( $2 \mathrm{~mol} \%)$ at $50{ }^{\circ} \mathrm{C}$ for $3 \mathrm{hrs}$ in the presence of metallic mercury were performed, from which hydrosilylated product was obtained selectively in $83 \%$ yield. Similarly, hydrosilylation of $\alpha$-methylstyrene with PMDS mediated by $\mathrm{Co}(\mathrm{OPv})_{2}(1 \mathrm{~mol} \%)$ and $\mathrm{CNAd}(3 \mathrm{~mol} \%)$ at $80{ }^{\circ} \mathrm{C}$ for $3 \mathrm{hrs}$ in the presence of metallic mercury also gave the hydrosilylated product selectively in $86 \%$ yield. It seems that the results did not support the catalysis of metal nanoparticles. However, it should be noted that this is not a convincing experiment to rule out the involvement of iron nanoparticle. Holland and Macleod reported that iron does not form an amalgam. (Macleod, K. C.; Holland, P. L, Nat. Chem., 2013, 5, 559-565).

\section{Comments to the catalyst precursors}

As for the reason why metal carboxylates are useful for the catalyst precursor of the catalytic hydrosilylation, we have the following working hypothesis at present stage. The detailed experiments to identify the catalyst species are needed to discuss the mechanisms, which are now under study. As described in Table S3 and S4, metal carboxylates shows high catalytic activity for the hydrosilylation of styrene or $\alpha$-methylstyrene with PMDS, whereas no reaction took place by using metal halides as catalyst under the same conditions. We found that the presence of M-O bond is crucial to generate active species. Besides metal carboxylates, metal alkoxides like $\mathrm{Co}\left(\mathrm{O}^{i} \mathrm{Pr}\right)_{2}$ showed catalytic activity toward hydrosilylation of $\alpha$-methylstyrene with PMDS at $80{ }^{\circ} \mathrm{C}$ for 6 hrs. We consider that strong affinity of silicon to oxygen would facilitate the generation of active species. One of the reasonable explanation is metathesis of a M-O bond and a $\mathrm{Si}-\mathrm{H}$ bond of hydrosilanes to form $\mathrm{M}-\mathrm{H}$ and $\mathrm{Si}-\mathrm{O}$ moieties. It is known that a metal hydride species promotes alkene isomerization, and hydrosilylation through Chalk-Harrod cycle. Because halides are strongly bound to the metal center, a stronger nucleophilic activator than hydrosilanes like 9-BBN is needed for the activation.

\section{Preliminary experiments on the functional group compatibility}

As reported in the text, the present hydrosilylation is tolerant to ethers and esters. Allylic amines can be used as the substrate to give the corresponding $\gamma$-aminosilanes, but the yield was moderate due to the slow reaction of enamines, which were formed by alkene isomerization of allylic amines, with hydrosiloxanes. Further experiments were performed to look at competitive experiments of styrene $\left[\mathrm{Fe}(\mathrm{OPv})_{2} / \mathrm{CNAd}\right.$ catalyst $]$ or $\alpha$-methylstyrene $\left[\mathrm{Co}(\mathrm{OPv})_{2} / \mathrm{CNAd}\right.$ catalyst] with PMDS in the presence of several functional groups. The results showed that the reaction of benzaldehyde with hydrosiloxanes were faster than hydrosilylation of the alkenes, whereas that of acetophenone was competitive. Ethyl acetate and N-methylpyrrodilinone did not disturb the hydrosilylation.

A possible chemoselectivity which may be visible between a $\mathrm{C}=\mathrm{C}$ bond conjugated to a benzene ring and that of an unconjugated $\mathrm{C}=\mathrm{C}$ bond was examined by competitive experiments of $\alpha$-methylstyrene $\left[\mathrm{Co}(\mathrm{OPv})_{2} / \mathrm{CNAd}\right.$ catalyst] with PMDS (2.6 eq. to a mixture of alkenes) in the presence of 1 -octene ( 1 eq. to $\alpha$-methylstyrene). The results are not straightforward. The results showed that the alkene isomerization took place quickly to form internal octenes, which were slowly converted to $\mathrm{Me}_{3} \mathrm{SiOMe}_{2} \mathrm{Si}-\left(\mathrm{CH}_{2}\right)_{7} \mathrm{CH}_{3}$. The rate of $\alpha$-methylstyrene was 
somewhat higher than the hydrosilylation of internal octenes. Thus, a mixture of the hydrosilylated product of $\alpha$-methylstyrene (86\%), $\mathrm{Me}_{3} \mathrm{SiOMe} 2 \mathrm{Si}-\left(\mathrm{CH}_{2}\right)_{7} \mathrm{CH}_{3}(27 \%)$, and internal octenes was obtained.

These preliminary experiments suggest that the present hydrosilylation is tolerant to ethers, amines, esters, and amides, but not to aldehydes to ketones. Selectivity between conjugated and non-conjugated alkenes is difficult to be achieved with the present catalyst systems under the standard reaction conditions. However, we found that the rate and selectivity were sensitive on the metal, ligands, hydrosilanes, substrate, and reaction conditions. High functional group compatibility could be observed in the further study.

\section{Reference}

(1) Cho, S.-H.; Ma, B.; Nguyen, S.B. T.; Hupp, J. T.; Albrecht-Schmitt, T. E. Chem. Comm., 2006, 2563.

(2) Tanabiki, M.; Tsuchiya, K.; Kumanomido, Y.; Matsubara, K.; Motoyama, Y.; Nagashima, H. Organometallics, 2004, 23, 3976.

(3) Delamarche, E.; Donzel, C.; Kamounah, F. S.; Wolf, H.; Geissler, M.; Stutz, R.; -Winkel, P. ; Michel, S.B. Lamgmuir, 2003, 19, 8749-8758.

(4) Eremenko, L.; Kiskin, M. A.; Fomina, I. G.; Sidorov, A. A.; Aleksandrov, G. G.; Ikorskii, V. N.; Shvedenkov, Y. G.; Rakitin, Y. V; Novotortsev, V. M. J. Cluster Sci., 2005, 16, 331-351.

(5) Aeomí, G.; Batsanov, A. S.; Christian, P.; Helliwell, M.; Parkin, A.; Parsons, S.; Smith, A. A.; Timco, G. A.; Winpenny, R. E. P. Chem. Eur. J., 2003, 9, 5142-5161.

(6) Golubnichaya, M. A.; Sidorov, A. A.; Fomina, I. G.; Ponina, M. O.; Deomidov, S. M.; Negedov, S. E.; Eremenko, I. L.; Moiseev, I. I. Russ. Chem. Bull., 1999, 48, 1751-1756.

\section{Additional references for ref $\mathbf{4}$ in the manuscript}

(a) Nesmeyanov, A. N.; Freidlina, R. Kh.; Chukovskaya, E. C.; Petrova, R. G.; Belyavsky, A. B. Tetrahedron, 1962, 17, 61-68. (b) Kakiuchi, F.; Tanaka, Y.; Chatani, N.; Murai, S. J. Organomet. Chem. 1993, 456, 45-47. (c) Marciniec, B.; Majchrzak, M. Inorg. Chem. Commun., 2000, 3, 371-375. (d) Harrod, J. F.; Chalk, A. J. J. Am. Chem. Soc. 1965, 87, 1133-1133. (e) Chalk, A. J. J. Organomet. Chem., 1970, 21, 207-213. (f) Chatani, N.; Kodama, T.; Kajikawa, Y.; Murakami, H.; Kakiuchi, F.; Ikeda, S.; Murai, S. Chem. Lett., 2000, 14-15. (g) Brookhart, M.; Grant, B. E. J. Am. Chem. Soc. 1993, 115, 2151-2156.

\section{Additional references for ref 5 in the manuscript}

Tondreau, A. M.; Atienza, C. C. H.; Darmon, J. M.; Milsmann, C.; Hoyt, H. M.; Weller, K. J.; Nye, S. A.; Lewis, K. M.; Boyer, J.; Delis, J. G. P.; Lobkovsky, E.; Chirik, P. J. Organometallics 2012, 31, 4886-4893.

\section{Additional references for ref 12 in the manuscript}

(a) Greenhalgh, M. D.; Jones, A. S.; Thomas, S. J. ChemCatChem 2015, 7, 190-222. (b) Kamata, K.; Suzuki, A.; Nakai, Y.; Nakazawa, H. Organometallics 2012, 31, 3825-3828. (c) Chen, J.; Cheng, B.; Cao, M.; Lu, Z. Angew. Chem. Int. Ed., 2015, 54, 4661-4664. (d) Peng, D.; Zhang, Y.; Du, X.; Zhang, L.; Leng, X.; Walter, M. D.; Huang, Z. J. Am. Chem. Soc., 2013, 135, 19154-19166 
Additional references for ref $\mathbf{1 3}$ in the manuscript

(a) Brandstadt, K.; Cook, S.; Nguyen, B. T.; Surgenor, A.; Taylor, R.; Tzou, M. WO Patent Application 2013043912 A2 to Dow Corning Corporation, priority date Sept 20, 2011. (b) Brandstadt, K.; Cook, S.; Nguyen, B.T.; Surgenor, A.; Taylor, R.; Tzou, M. WO Patent Application 2013043783 A2 to Dow Corning Corporation, priority date Sept 20, 2011.

Additional references for ref 15 in the manuscript

(a) Furuta, A.; Nishiyama, H. Tetrahedron Lett. 2008, 49, 110-113. (b) Inagaki, T.; Phong, L. T.; Furuta, A.; Ito, J.; Nishiyama, H. Chem. Eur. J. 2010, 16, 3090-3096. 
Figure S5-1. ${ }^{1} \mathrm{H}$ NMR spectrum of a solution of $1 \mathrm{a}$ in $\mathrm{CDCl}_{3}$ at room temperature
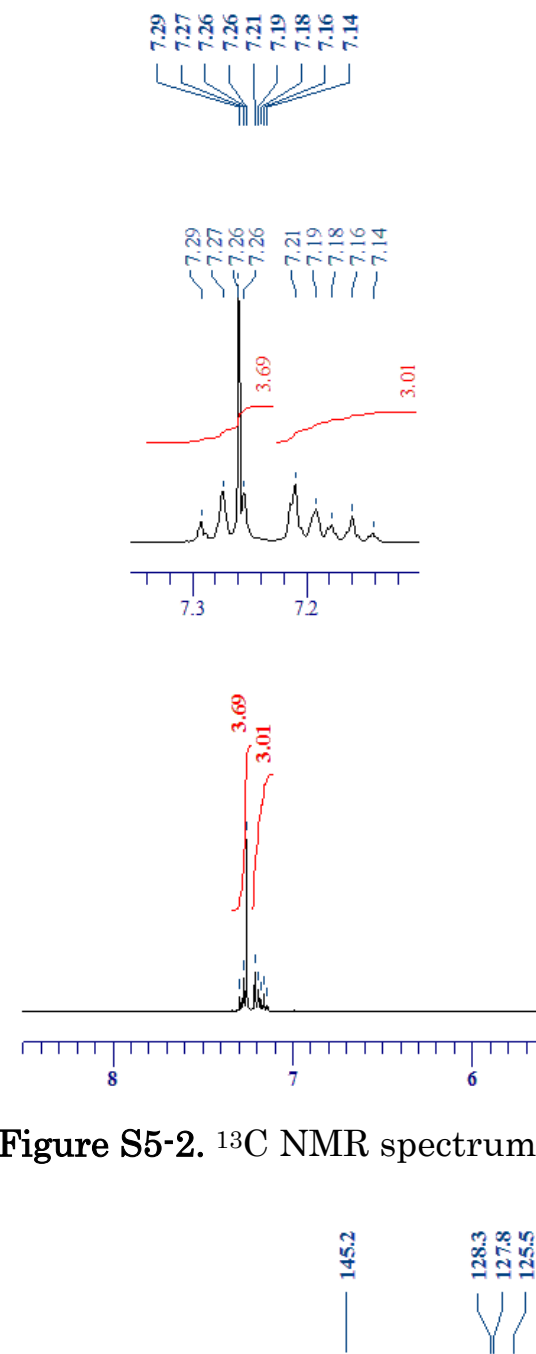

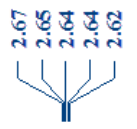

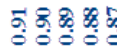

111
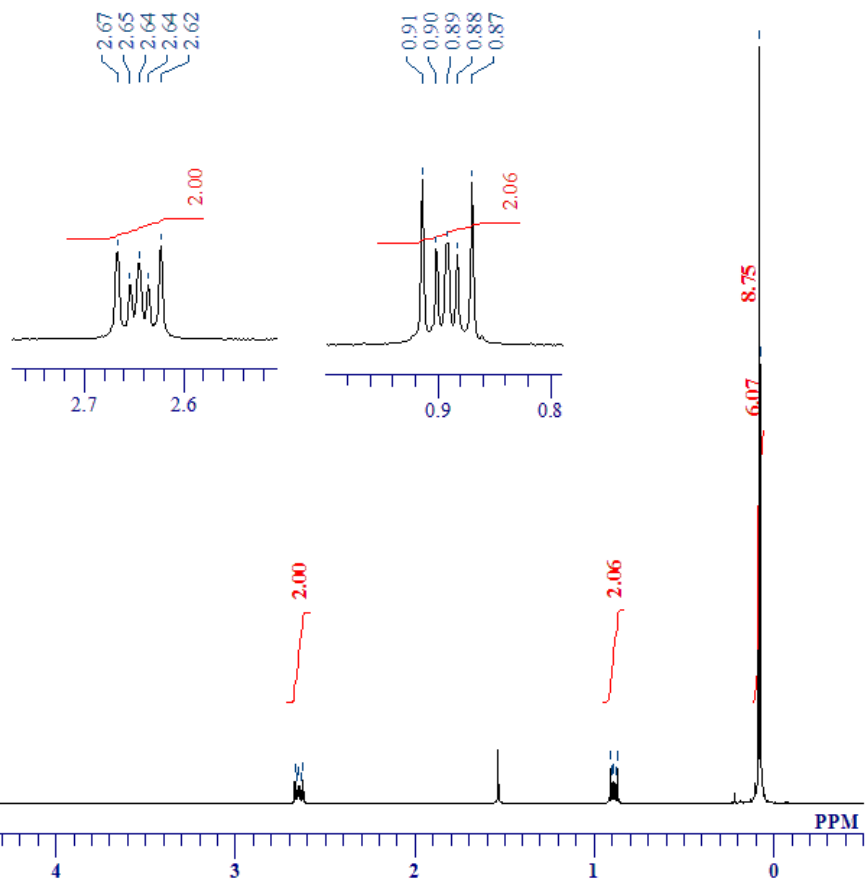

Figure S5-2. ${ }^{13} \mathrm{C}$ NMR spectrum of a solution of $1 \mathrm{a}$ in $\mathrm{CDCl}_{3}$ at room temperature

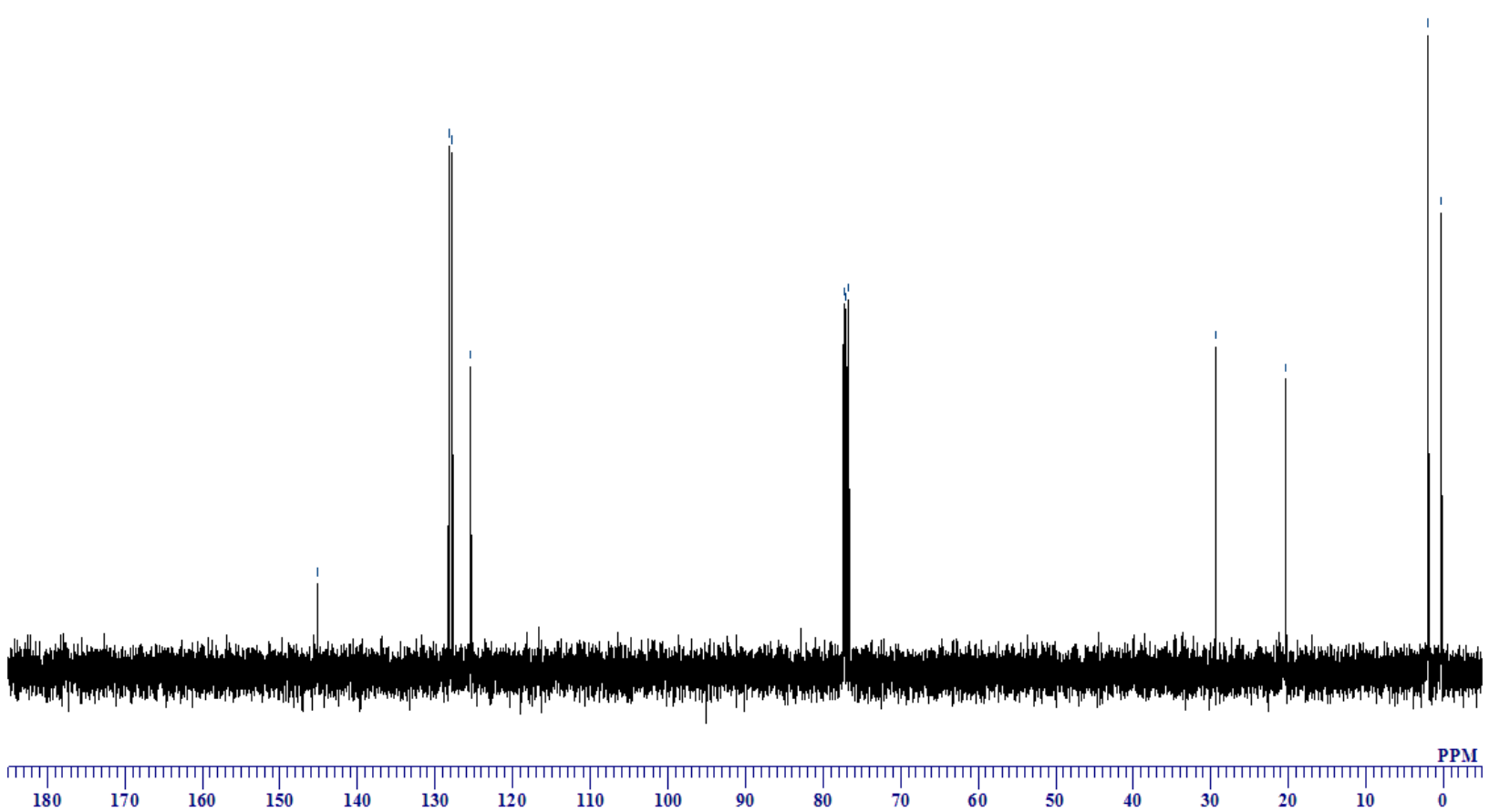


Figure S5-3. ${ }^{29} \mathrm{Si}$ NMR spectrum of a solution of $1 \mathrm{a}$ in $\mathrm{CDCl}_{3}$ at room temperature

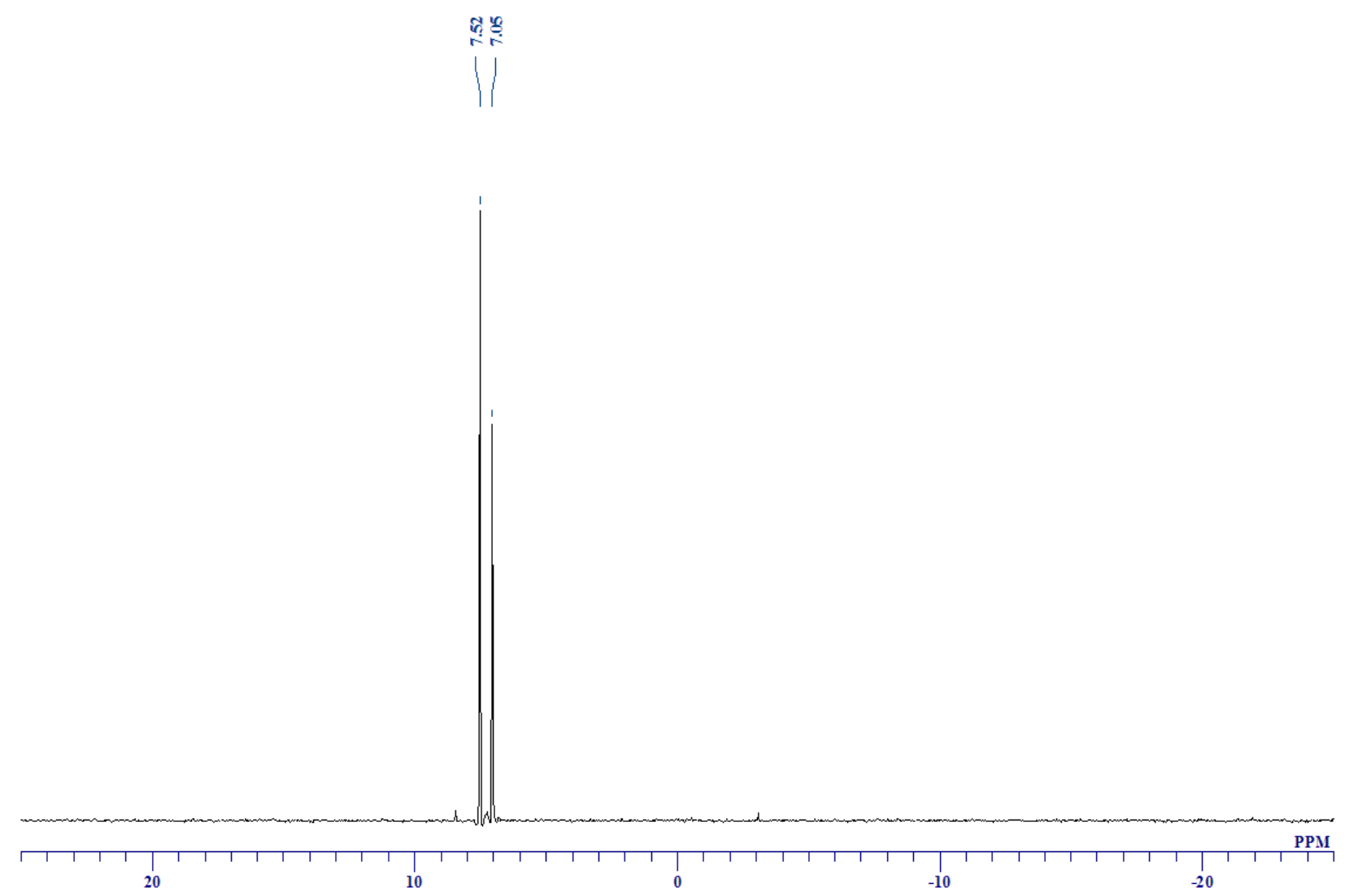

Figure S6-1. ${ }^{1} \mathrm{H}$ NMR spectrum of a solution of $2 \mathbf{a}$ in $\mathrm{CDCl}_{3}$ at room temperature

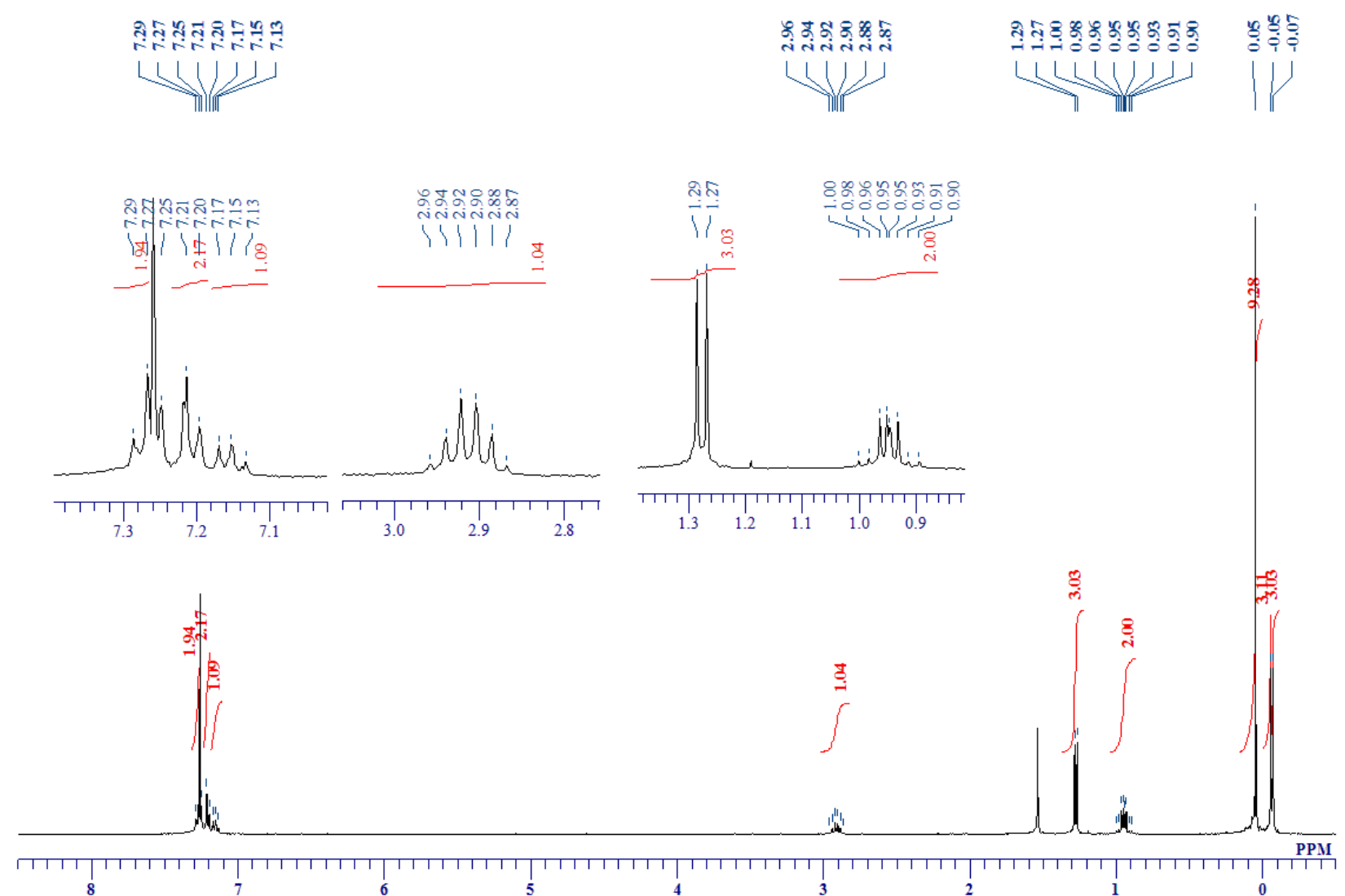


Figure S6-2. ${ }^{13} \mathrm{C}$ NMR spectrum of a solution of $2 \mathrm{a}$ in $\mathrm{CDCl}_{3}$ at room temperature

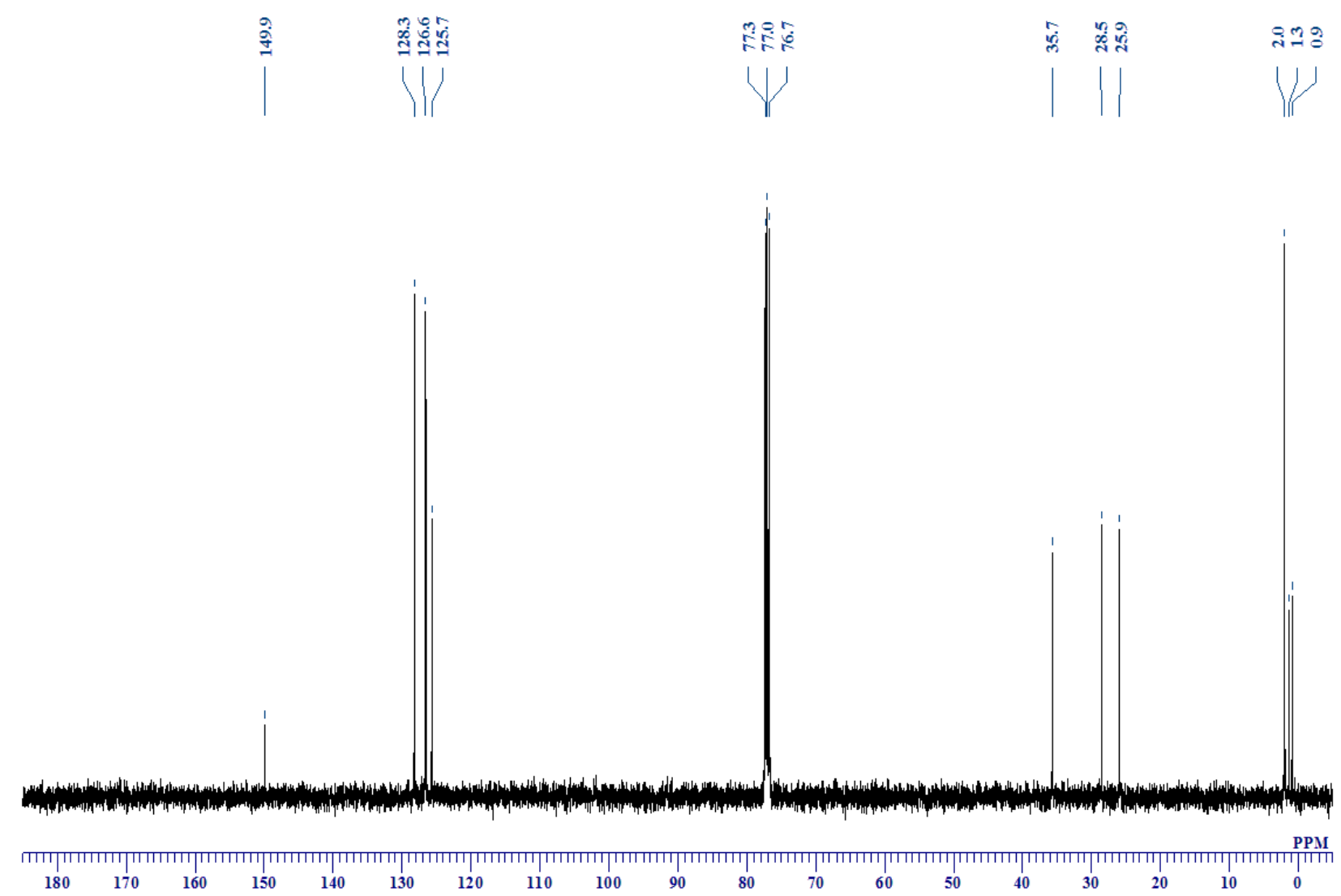

Figure S6-3. ${ }^{29} \mathrm{Si} \mathrm{NMR} \mathrm{spectrum} \mathrm{of} \mathrm{a} \mathrm{solution} \mathrm{of} \mathbf{2 a}$ in $\mathrm{CDCl}_{3}$ at room temperature<smiles></smiles>

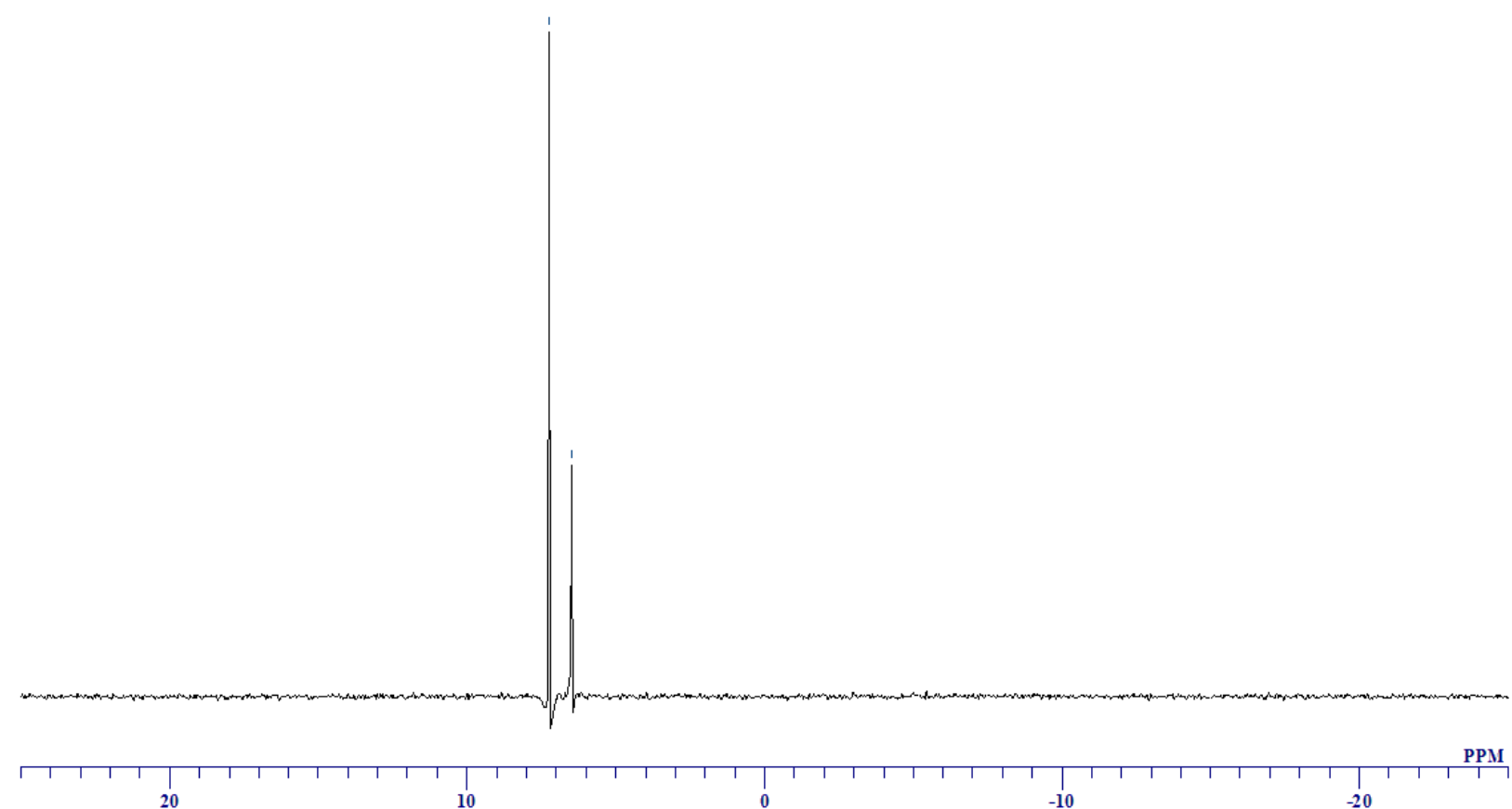


Figure S7-1. ${ }^{1} \mathrm{H}$ NMR spectrum of a solution of $3 \mathrm{a}$ in $\mathrm{CDCl}_{3}$ at room temperature ำำำำำำ

YV
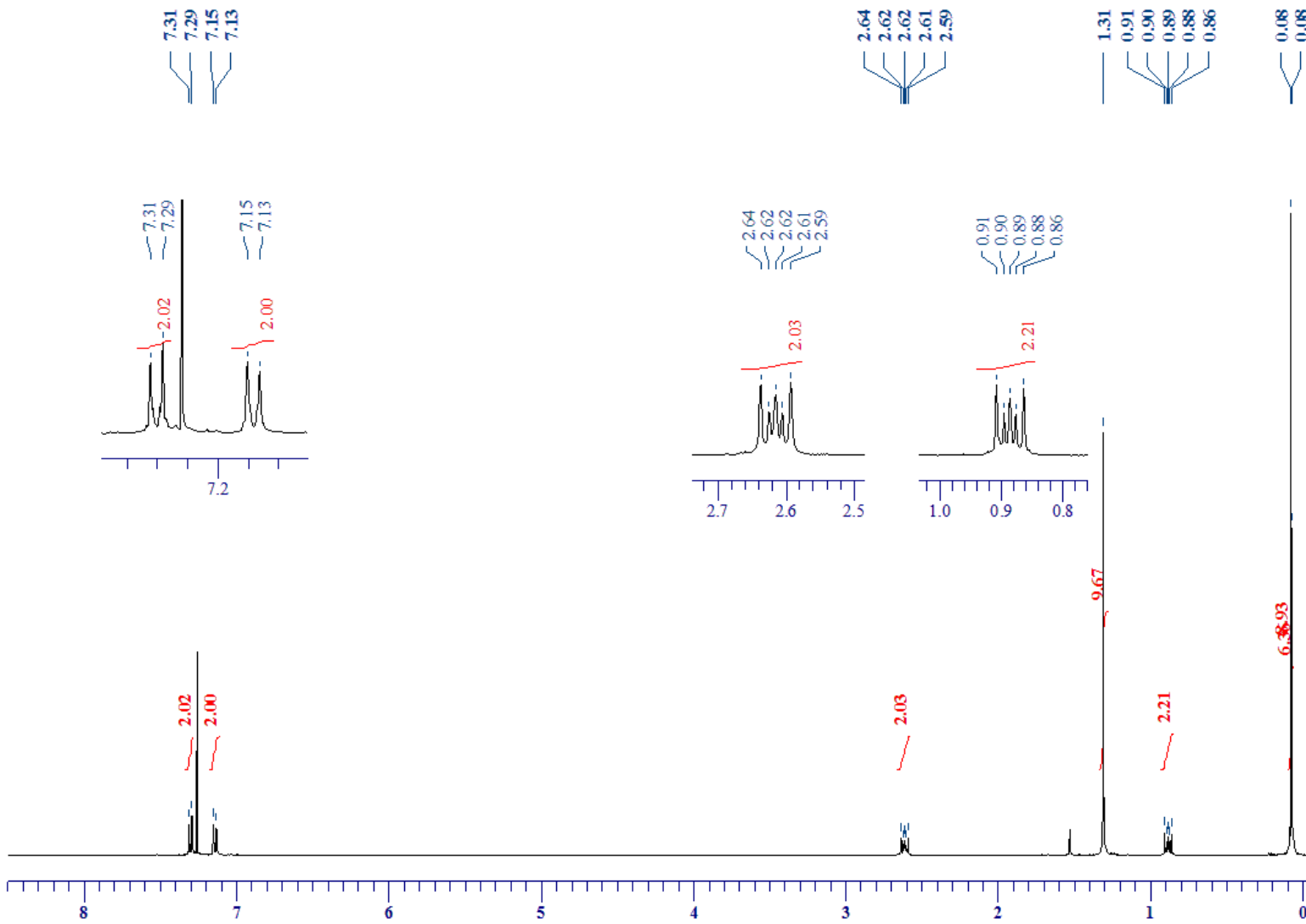

PPM

Figure S7-2. ${ }^{13} \mathrm{C}$ NMR spectrum of a solution of $3 \mathbf{a}$ in $\mathrm{CDCl}_{3}$ at room temperature
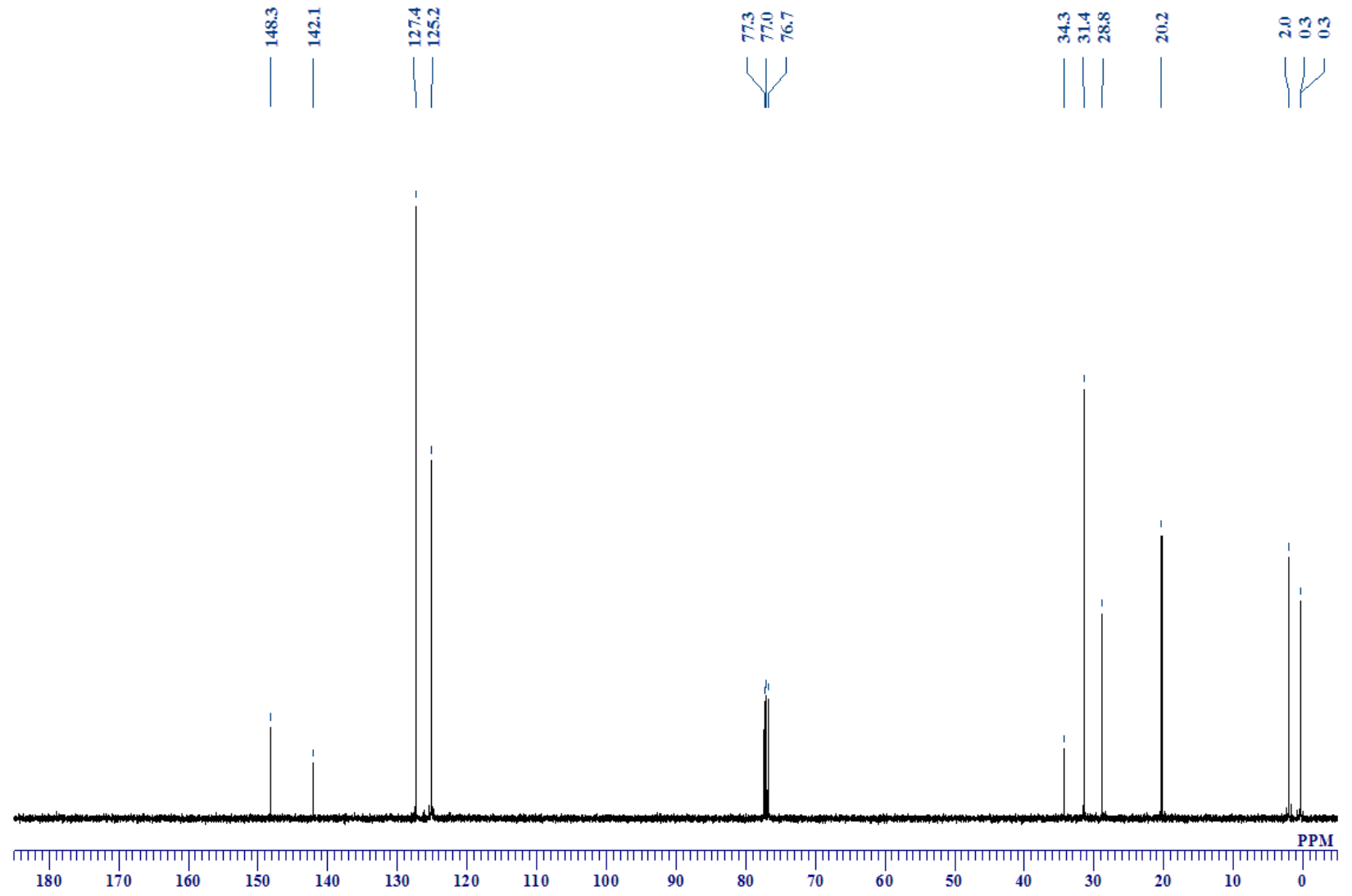
Figure S7-3. ${ }^{29} \mathrm{Si}$ NMR spectrum of a solution of $3 \mathrm{a}$ in $\mathrm{CDCl}_{3}$ at room temperature<smiles>[Te]#[W]</smiles>

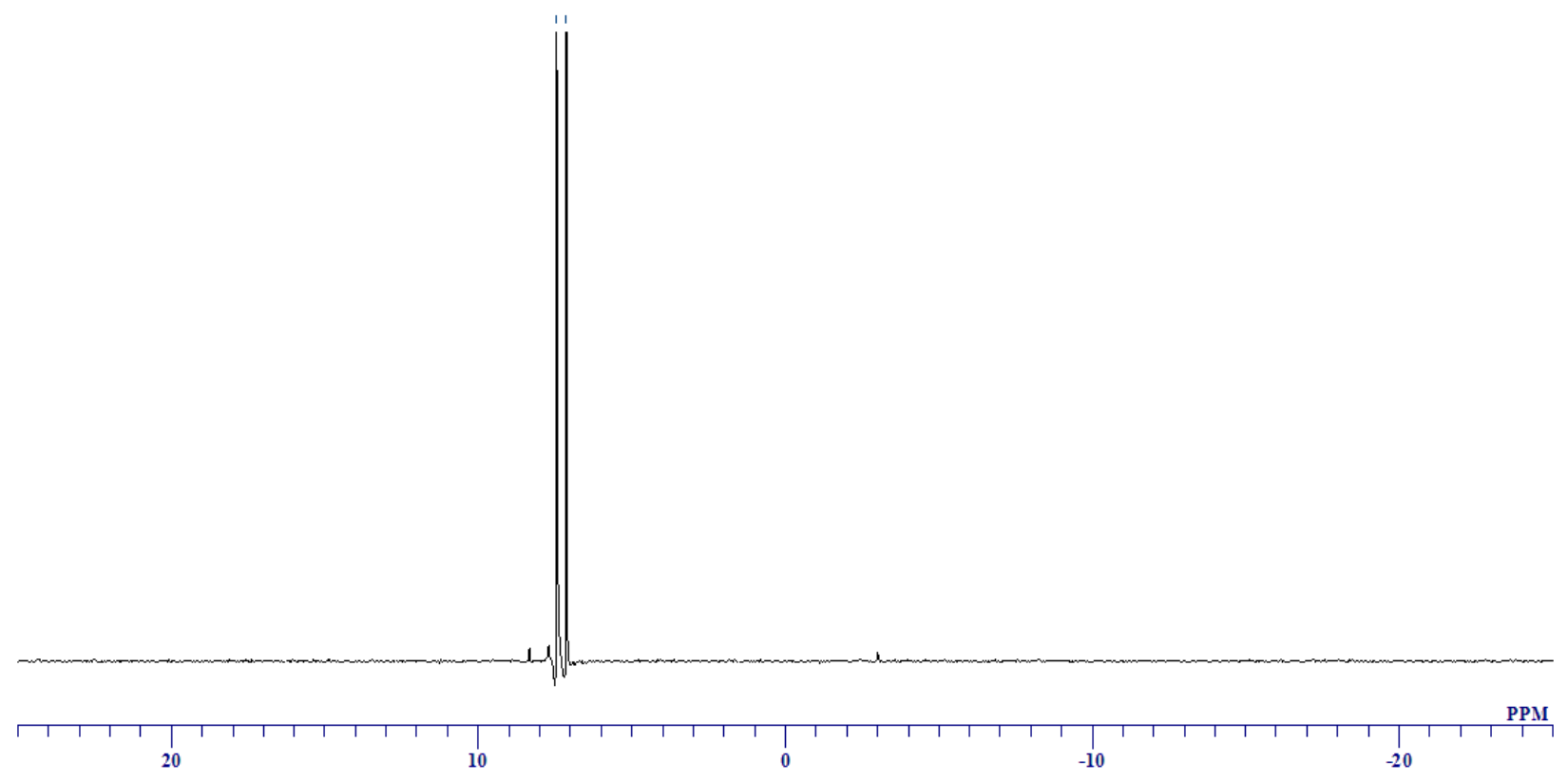

Figure S8-1. ${ }^{1} \mathrm{H}$ NMR spectrum of a solution of $4 \mathrm{a}$ in $\mathrm{CDCl}_{3}$ at room temperature

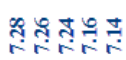
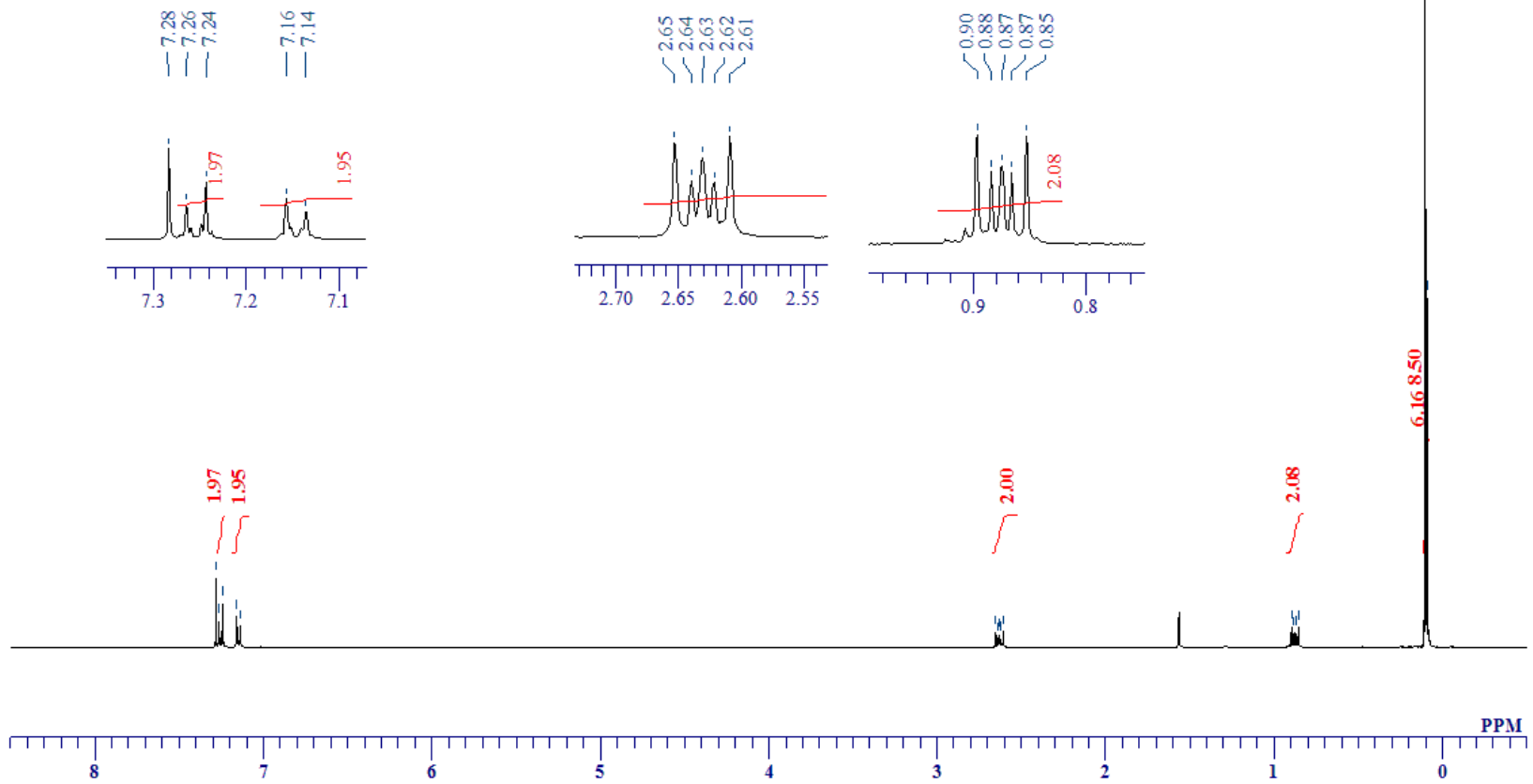
Figure S8-2. ${ }^{13} \mathrm{C}$ NMR spectrum of a solution of $4 \mathrm{a}$ in $\mathrm{CDCl}_{3}$ at room temperature
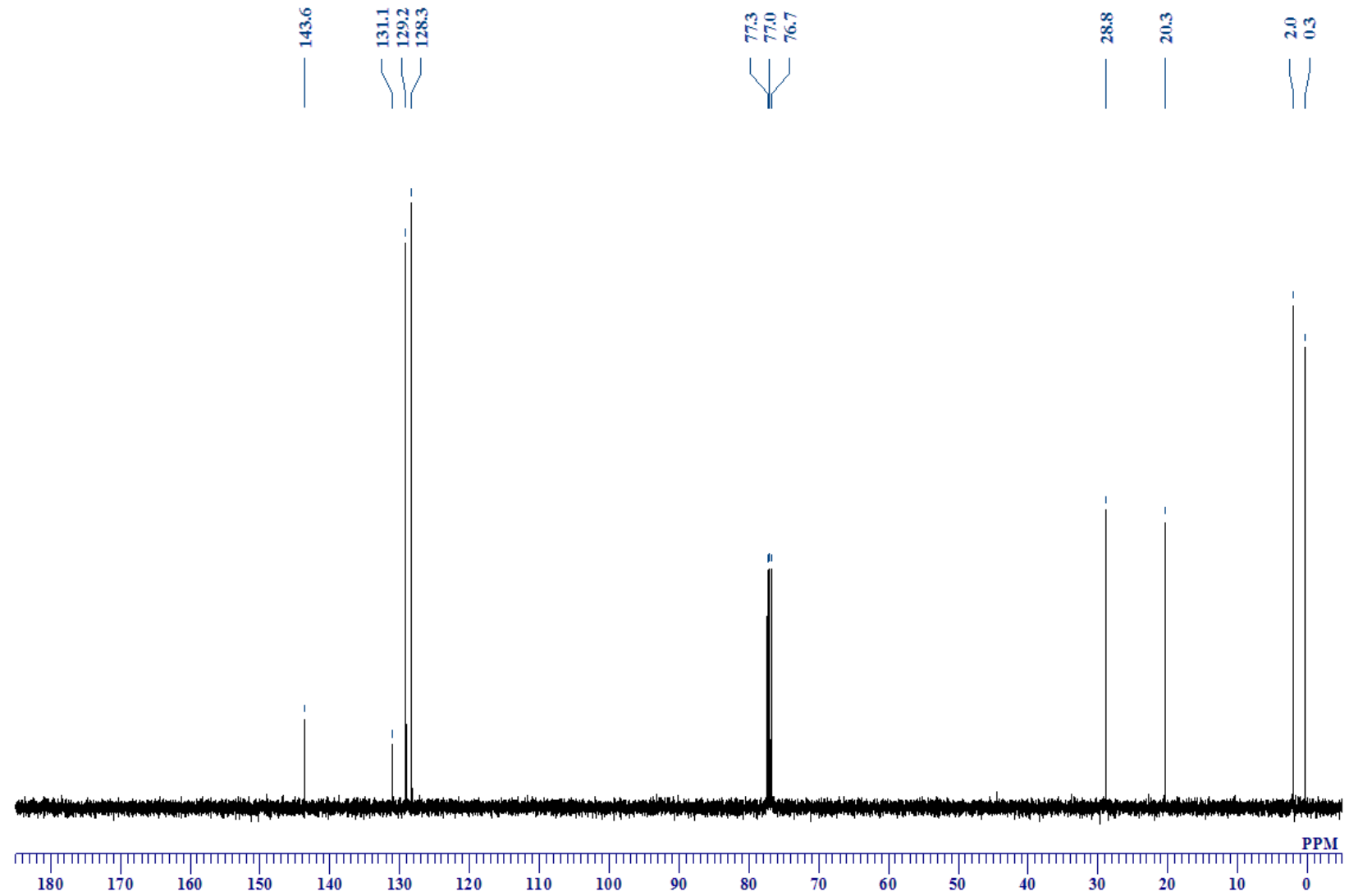

Figure S8-3. ${ }^{29} \mathrm{Si} \mathrm{NMR}$ spectrum of a solution of $\mathbf{4 a}$ in $\mathrm{CDCl}_{3}$ at room temperature

.

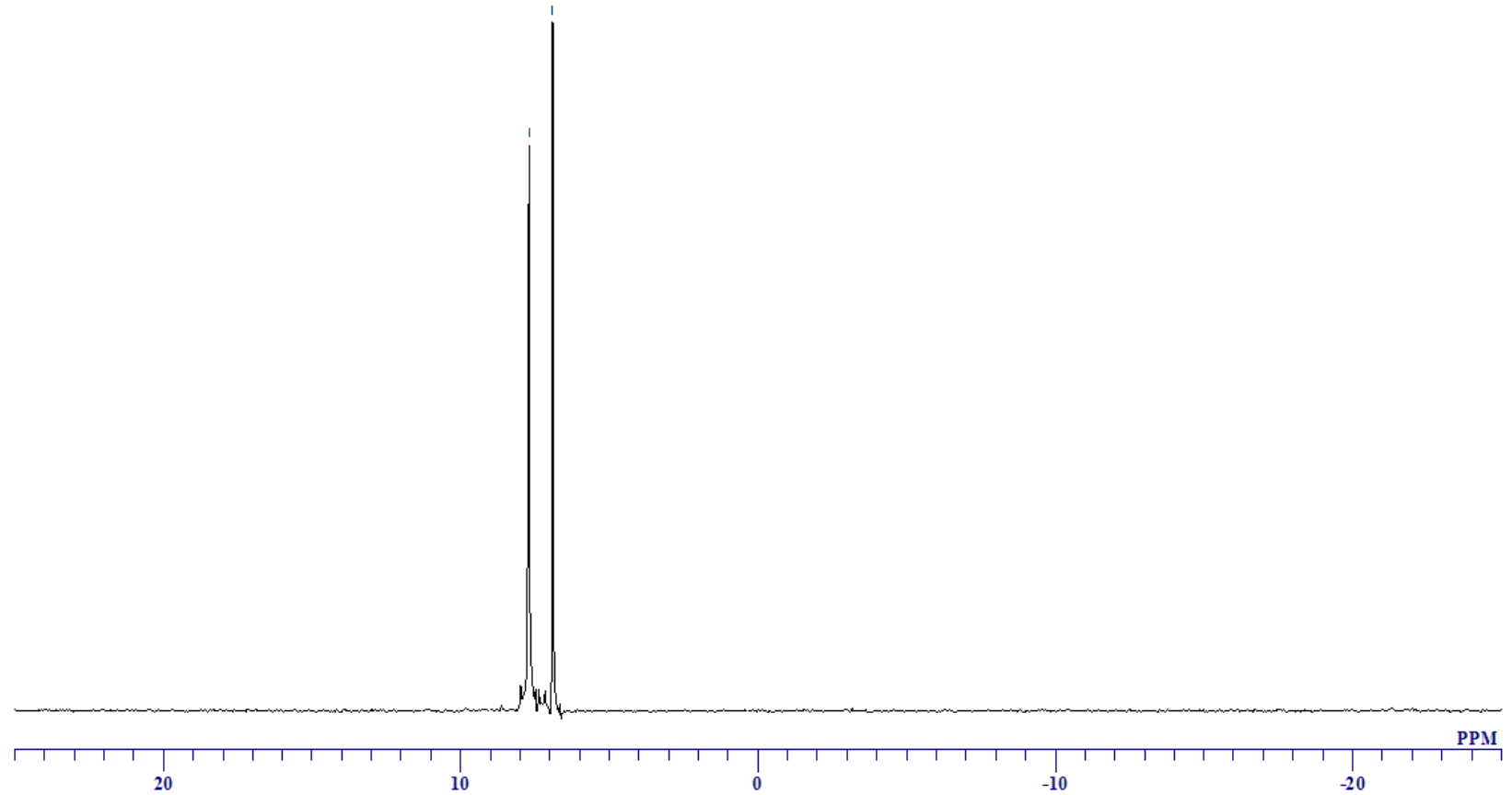


Figure S9-1. ${ }^{1} \mathrm{H}$ NMR spectrum of a solution of $\mathbf{5 a}$ in $\mathrm{CDCl}_{3}$ at room temperature

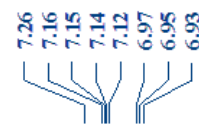

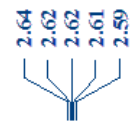
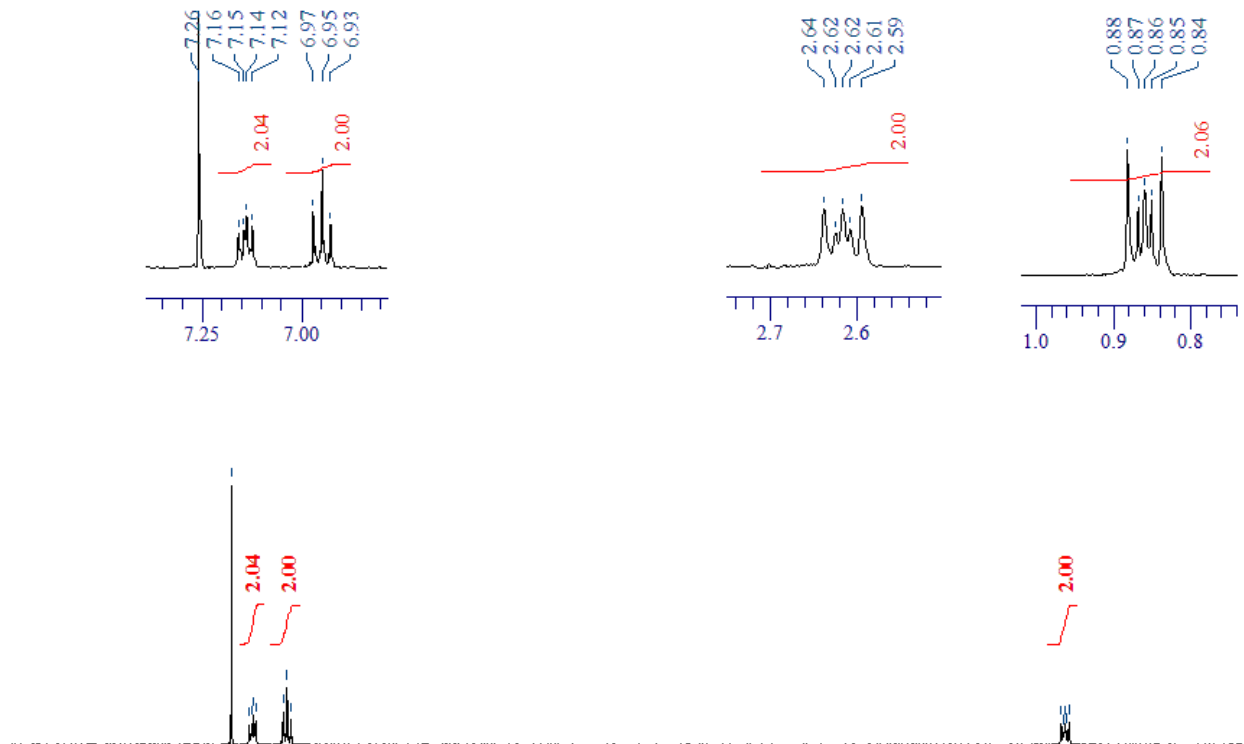

\section{$\int^{4}$ \\ $\int$ \\ il}

i

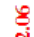

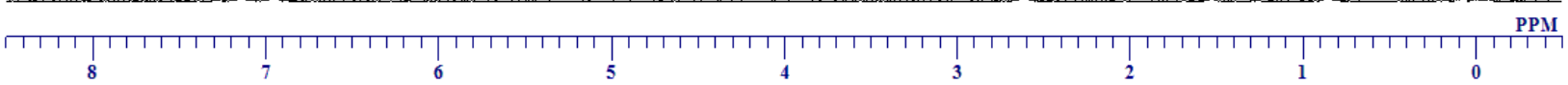

Figure S9-2. ${ }^{13} \mathrm{C}$ NMR spectrum of a solution of $5 \mathrm{a}$ in $\mathrm{CDCl}_{3}$ at room temperature

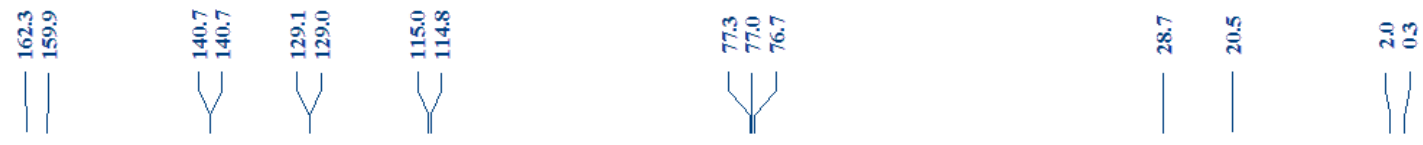

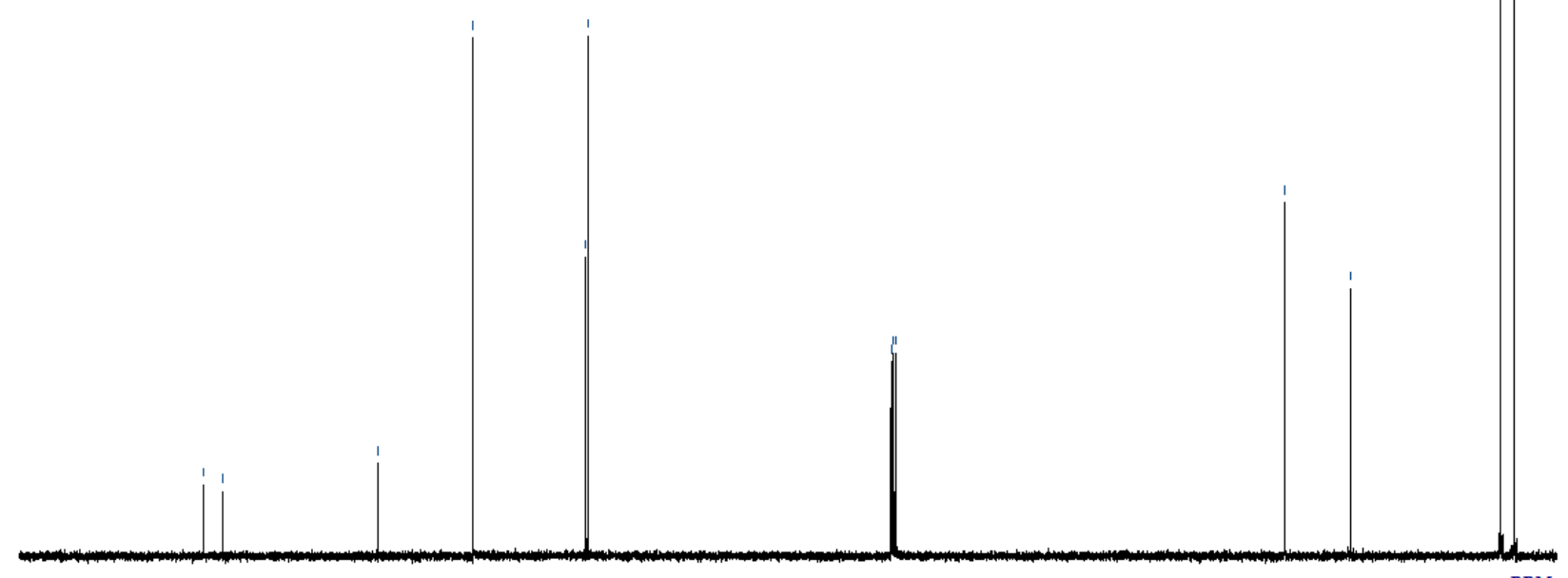

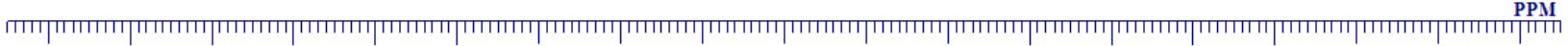

170

$160 \quad 150$

140

$130 \quad 12$

$110 \quad 100$ 
Figure S9-3. ${ }^{19} \mathrm{~F}$ NMR spectrum of a solution of $5 \mathbf{a}$ in $\mathrm{CDCl}_{3}$ at room temperature

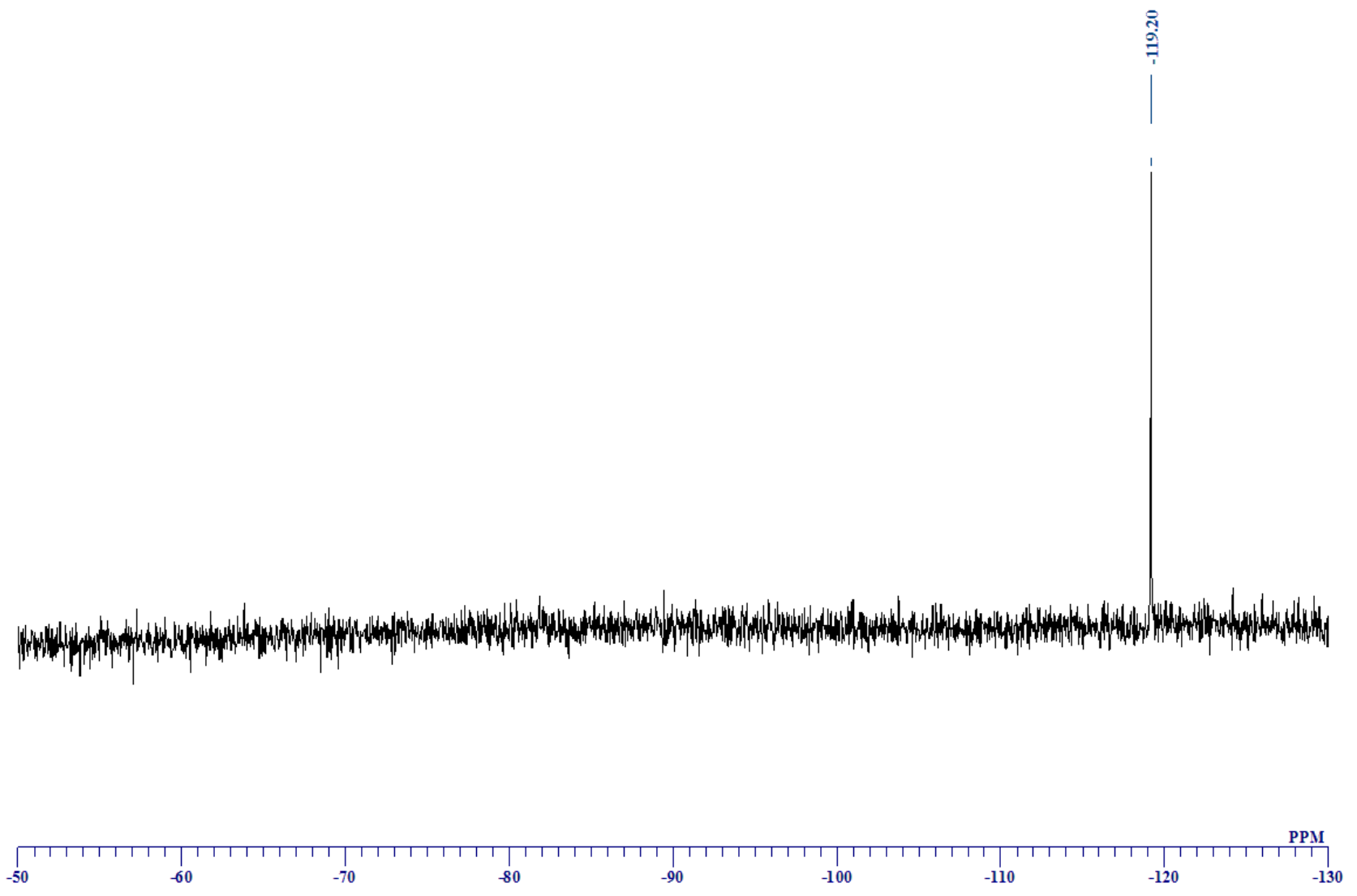

Figure S9-4. ${ }^{29} \mathrm{Si} \mathrm{NMR} \mathrm{spectrum} \mathrm{of} \mathrm{a} \mathrm{solution} \mathrm{of} \mathbf{5 a}$ in $\mathrm{CDCl}_{3}$ at room temperature

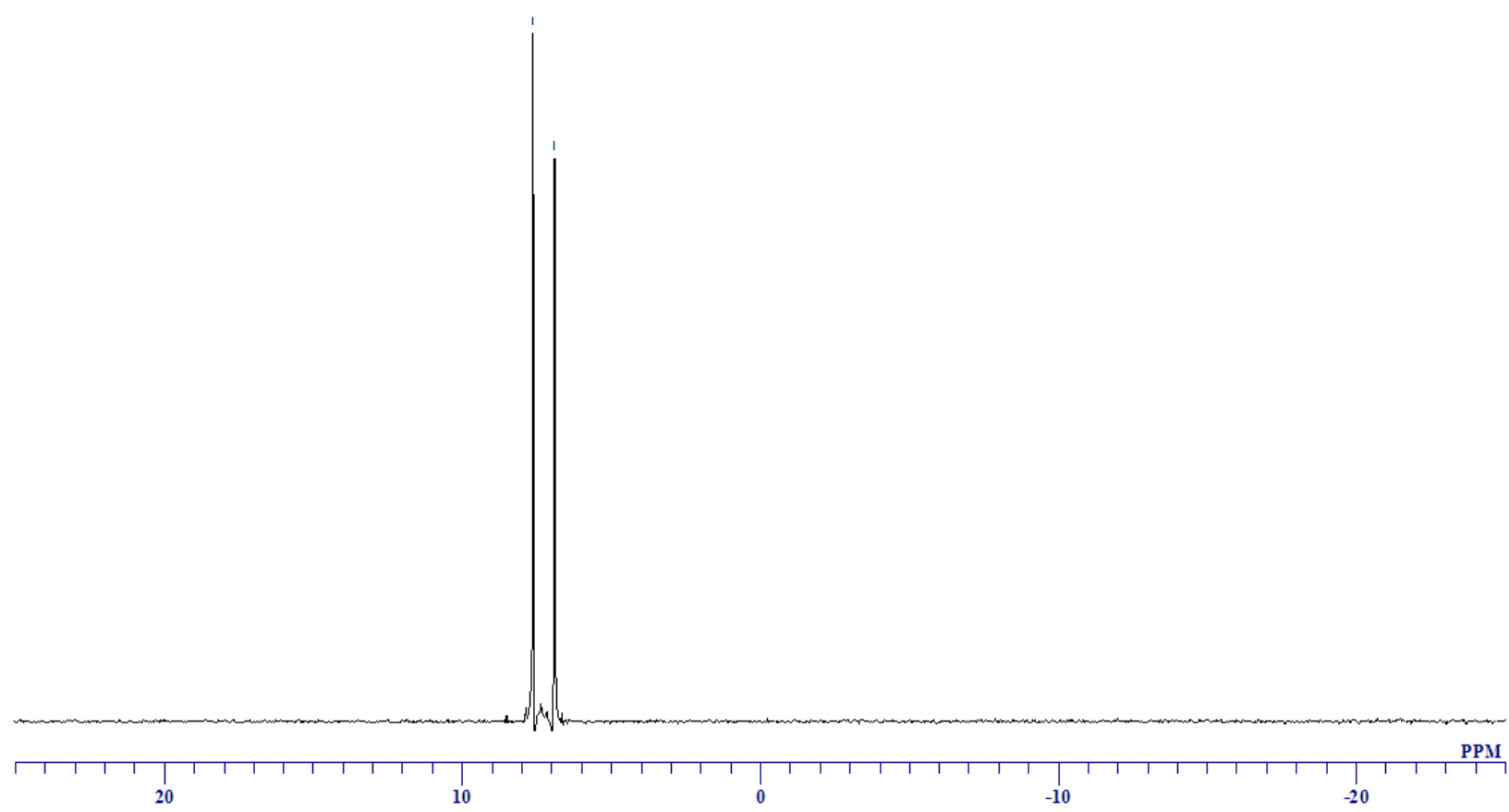


Figure S10-1. ${ }^{1} \mathrm{H}$ NMR spectrum of a solution of $6 \mathrm{a}$ in $\mathrm{CDCl}_{3}$ at room temperature
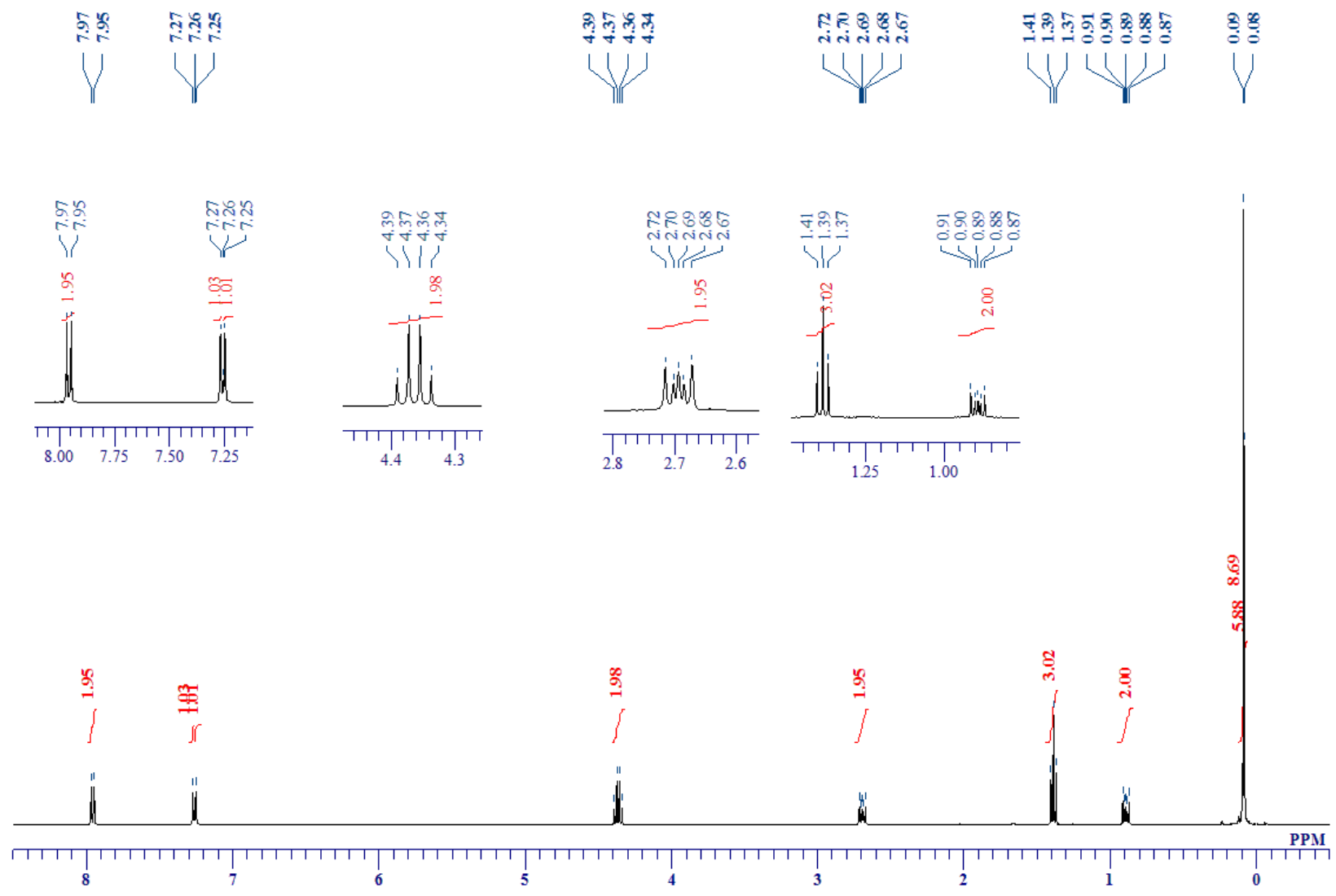

Figure $\mathrm{S} 10-2 .{ }^{13} \mathrm{C}$ NMR spectrum of a solution of $\mathbf{6 a}$ in $\mathrm{CDCl}_{3}$ at room temperature
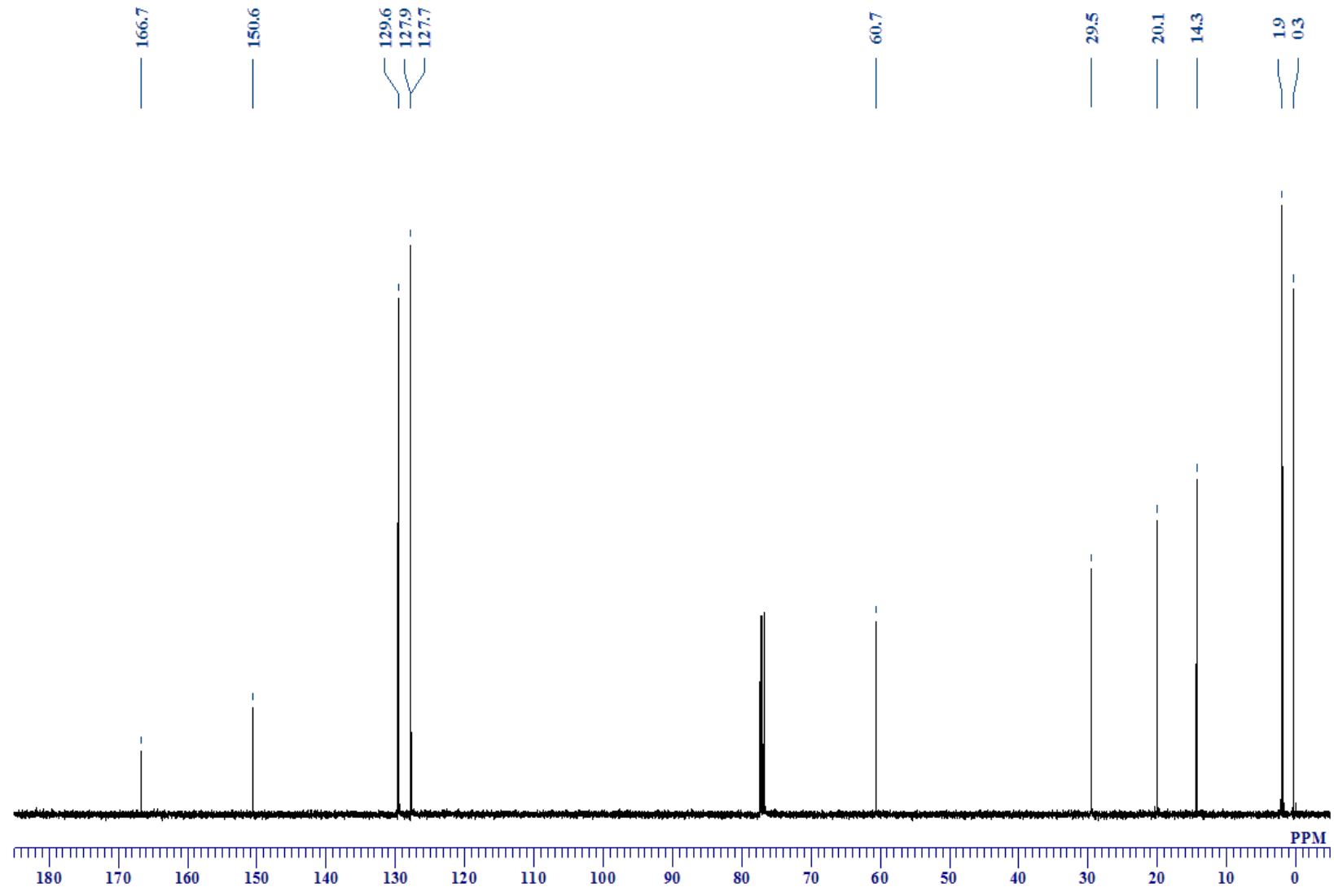
Figure S10-3. ${ }^{29} \mathrm{Si} \mathrm{NMR}$ spectrum of a solution of $6 \mathrm{a}$ in $\mathrm{CDCl}_{3}$ at room temperature

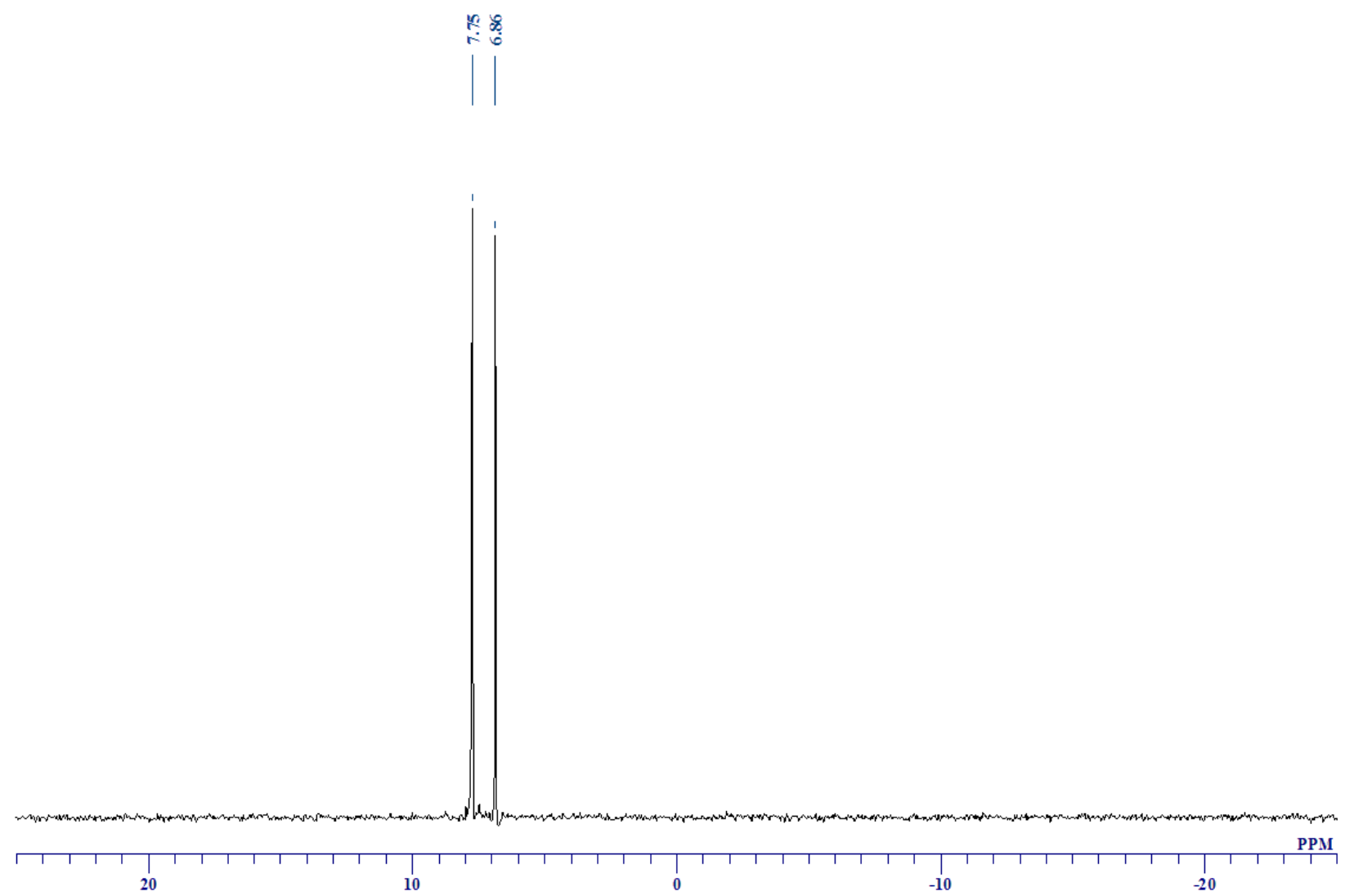

Figure $\mathrm{S} 11-1 .{ }^{1} \mathrm{H}$ NMR spectrum of a solution of $7 \mathrm{a}$ in $\mathrm{CDCl}_{3}$ at room temperature
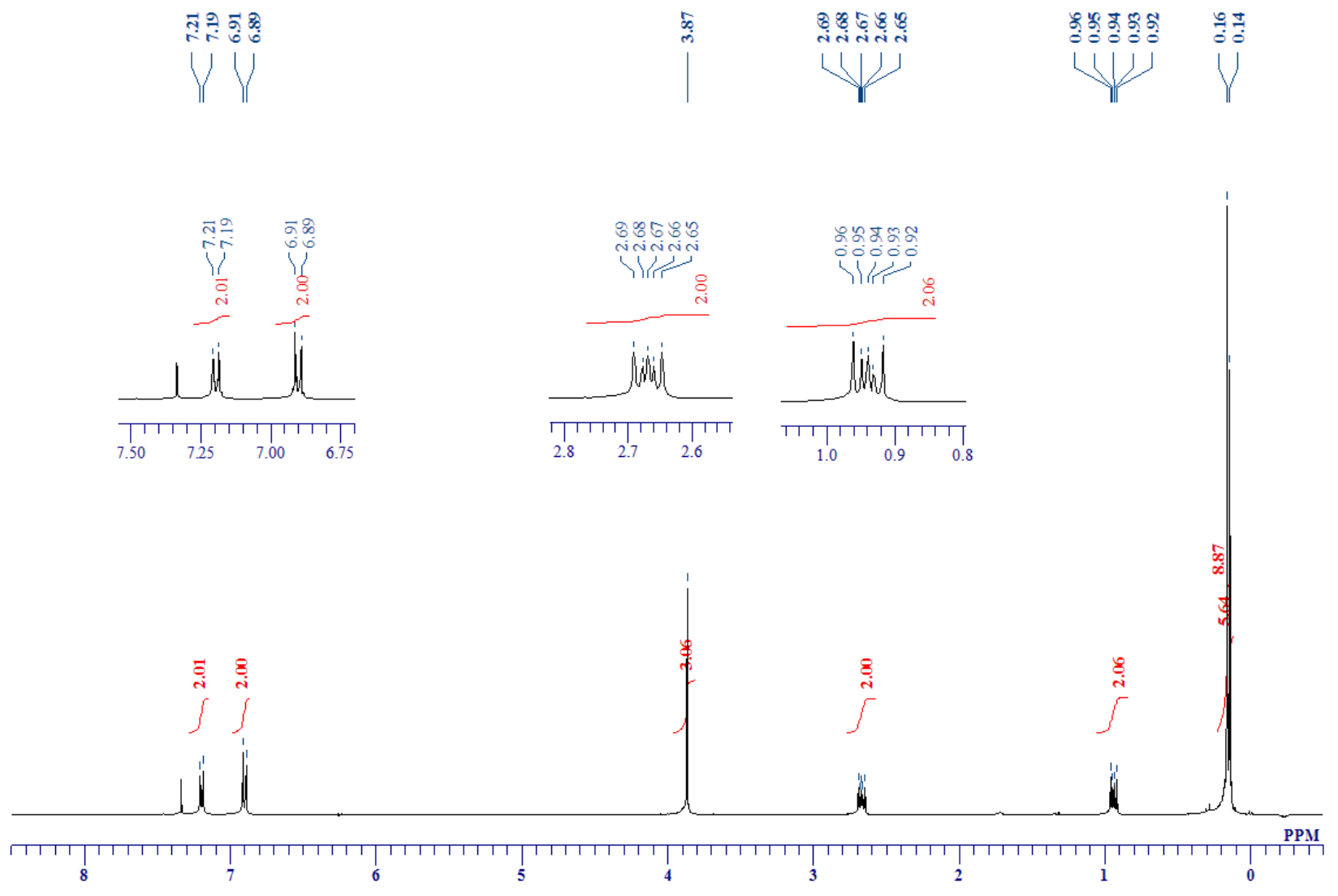
Figure $\mathrm{S} 11-2 .{ }^{13} \mathrm{C}$ NMR spectrum of a solution of $7 \mathbf{a}$ in $\mathrm{CDCl}_{3}$ at room temperature

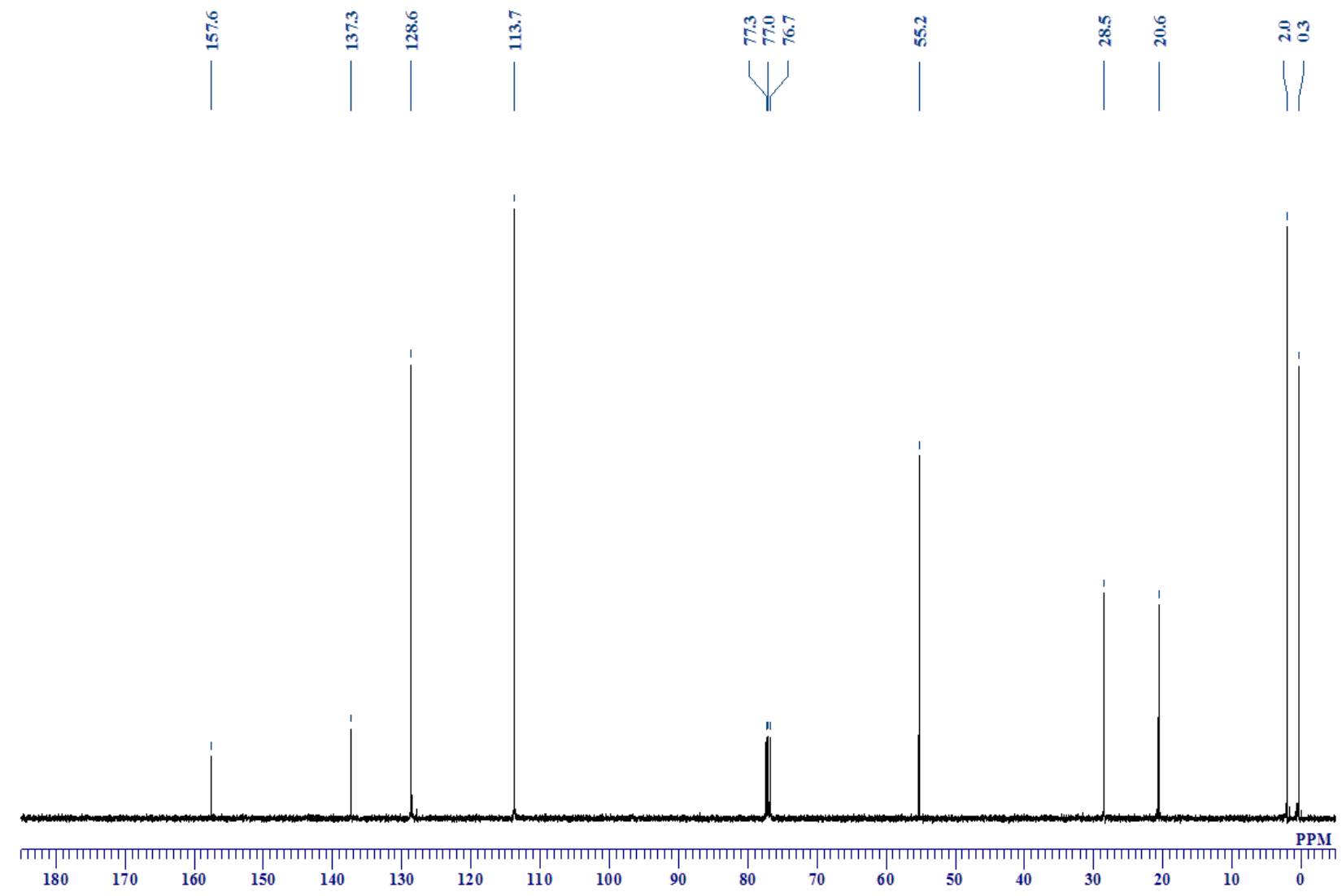

Figure S11-3. ${ }^{29} \mathrm{Si} \mathrm{NMR}$ spectrum of a solution of $7 \mathrm{a}$ in $\mathrm{CDCl}_{3}$ at room temperature<smiles>C1=C2C=[C+]1C2</smiles>

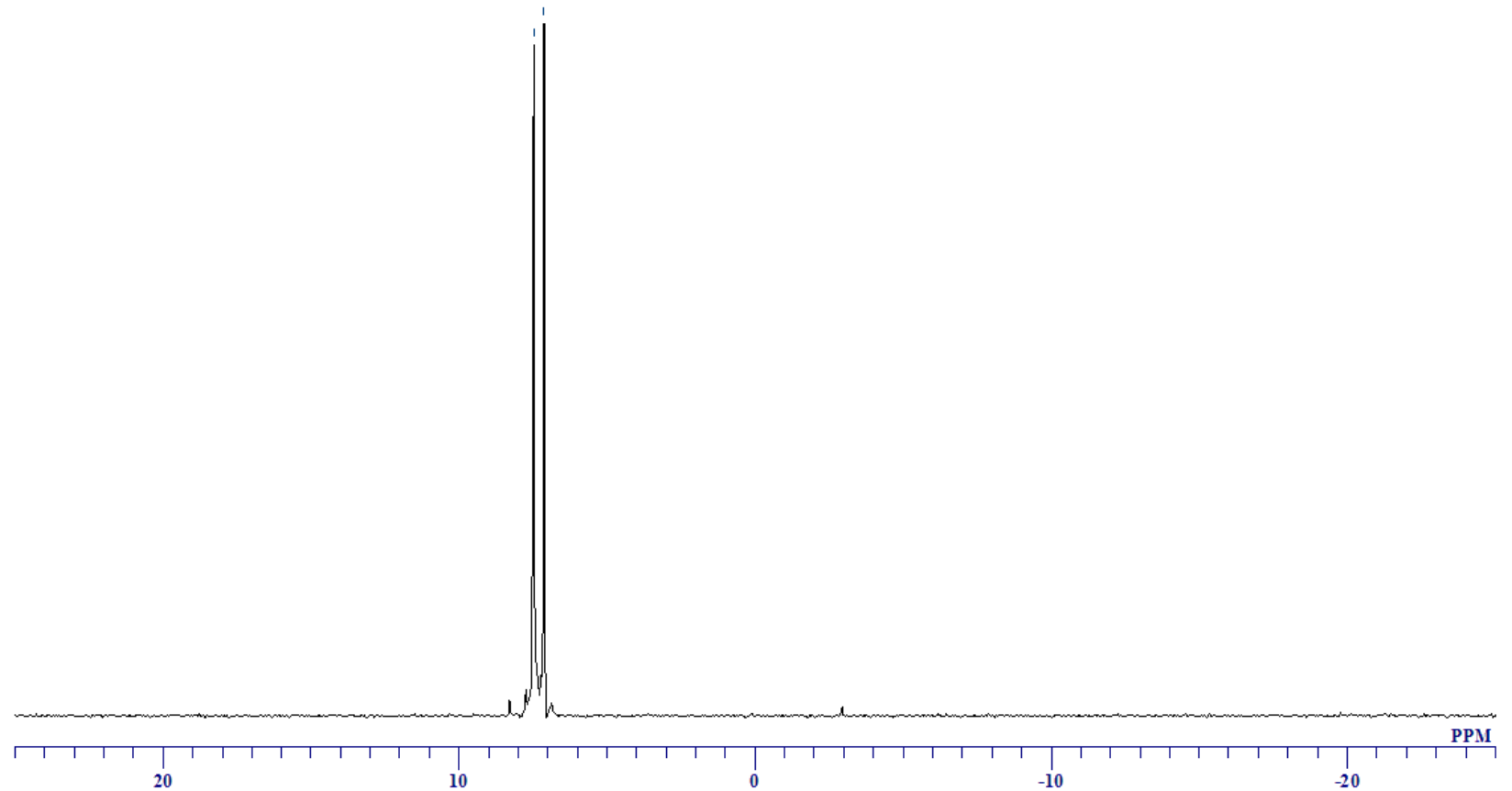


Figure S12-1. ${ }^{1} \mathrm{H}$ NMR spectrum of a solution of $8 \mathbf{a}$ in $\mathrm{CDCl}_{3}$ at room temperature
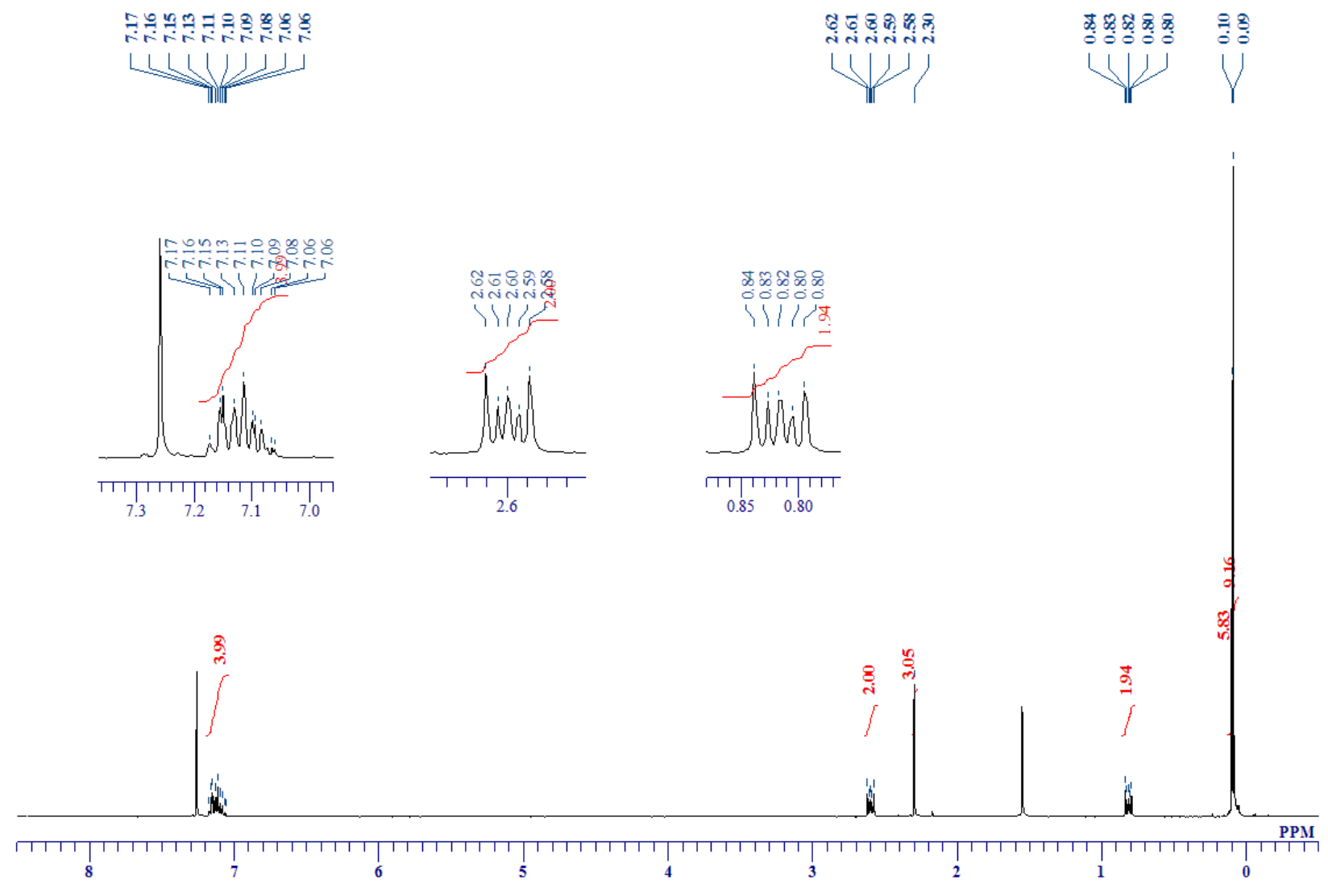

Figure $\mathrm{S} 12-2 .{ }^{13} \mathrm{C}$ NMR spectrum of a solution of $8 \mathbf{a}$ in $\mathrm{CDCl}_{3}$ at room temperature
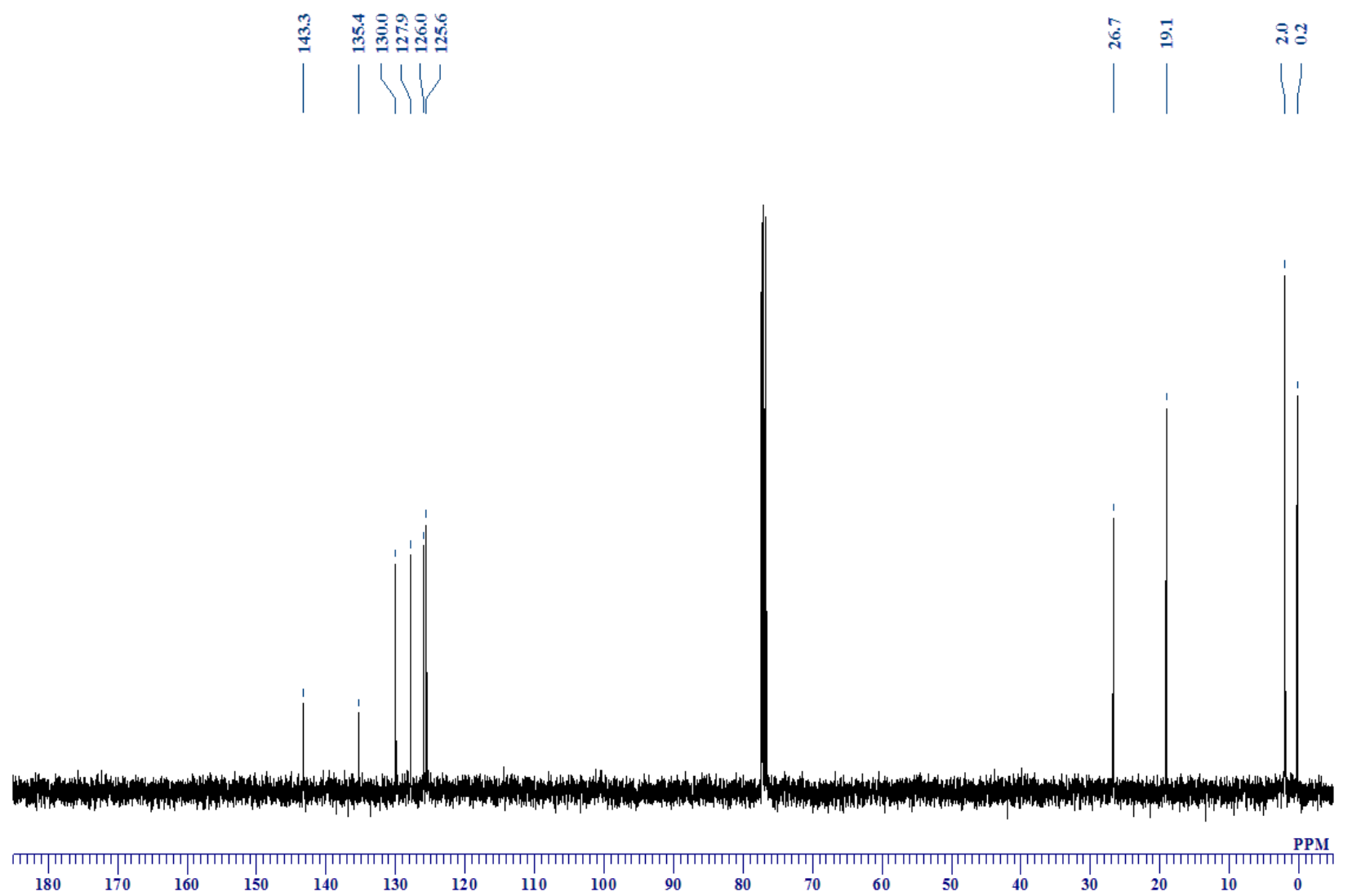
Figure S12-3. ${ }^{29} \mathrm{Si} \mathrm{NMR}$ spectrum of a solution of $8 \mathrm{a}$ in $\mathrm{CDCl}_{3}$ at room temperature

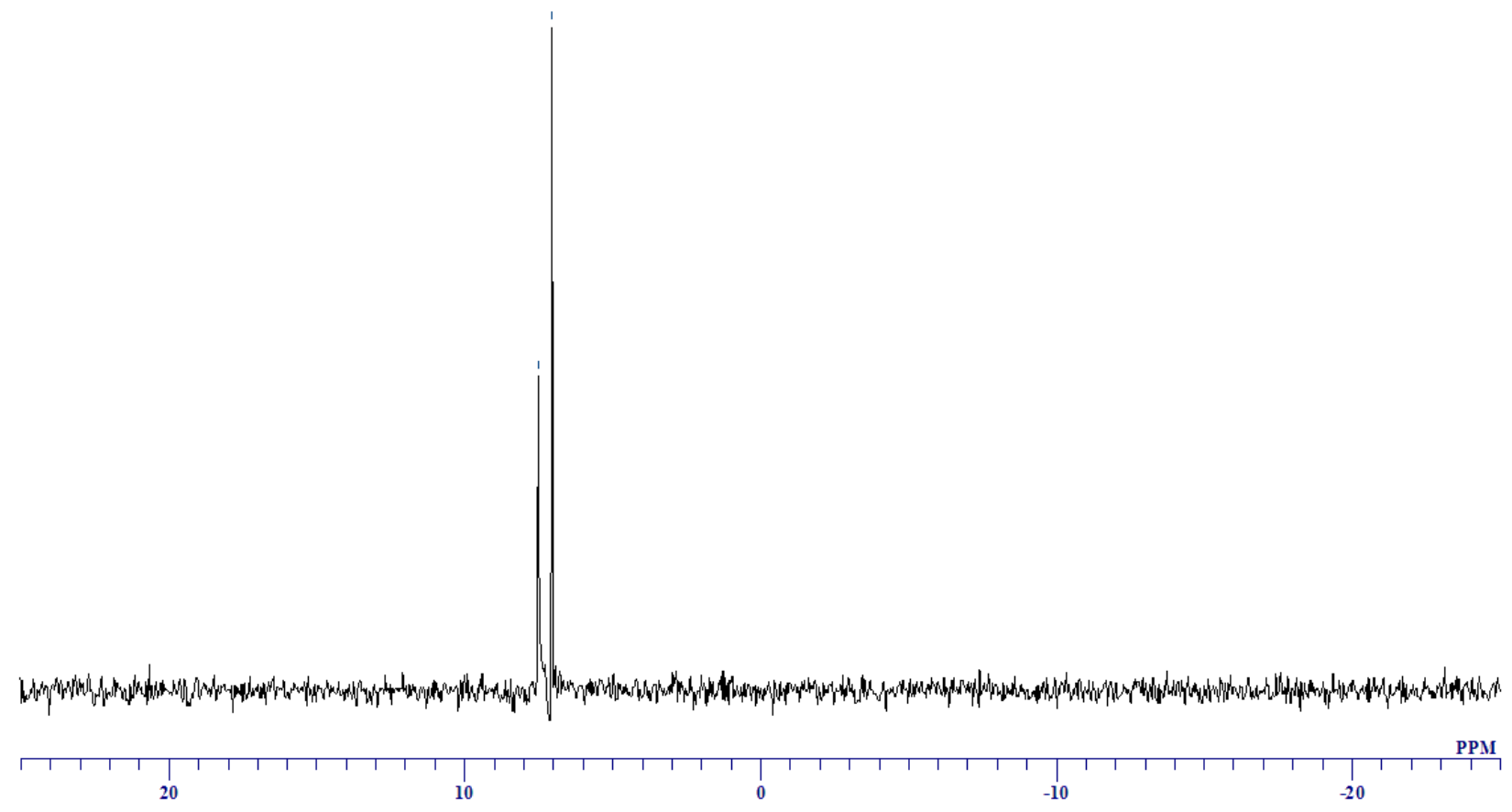

Figure $\mathbf{S} 13-1 .{ }^{1} \mathrm{H}$ NMR spectrum of a solution of $9 \mathbf{a}$ in $\mathrm{CDCl}_{3}$ at room temperature
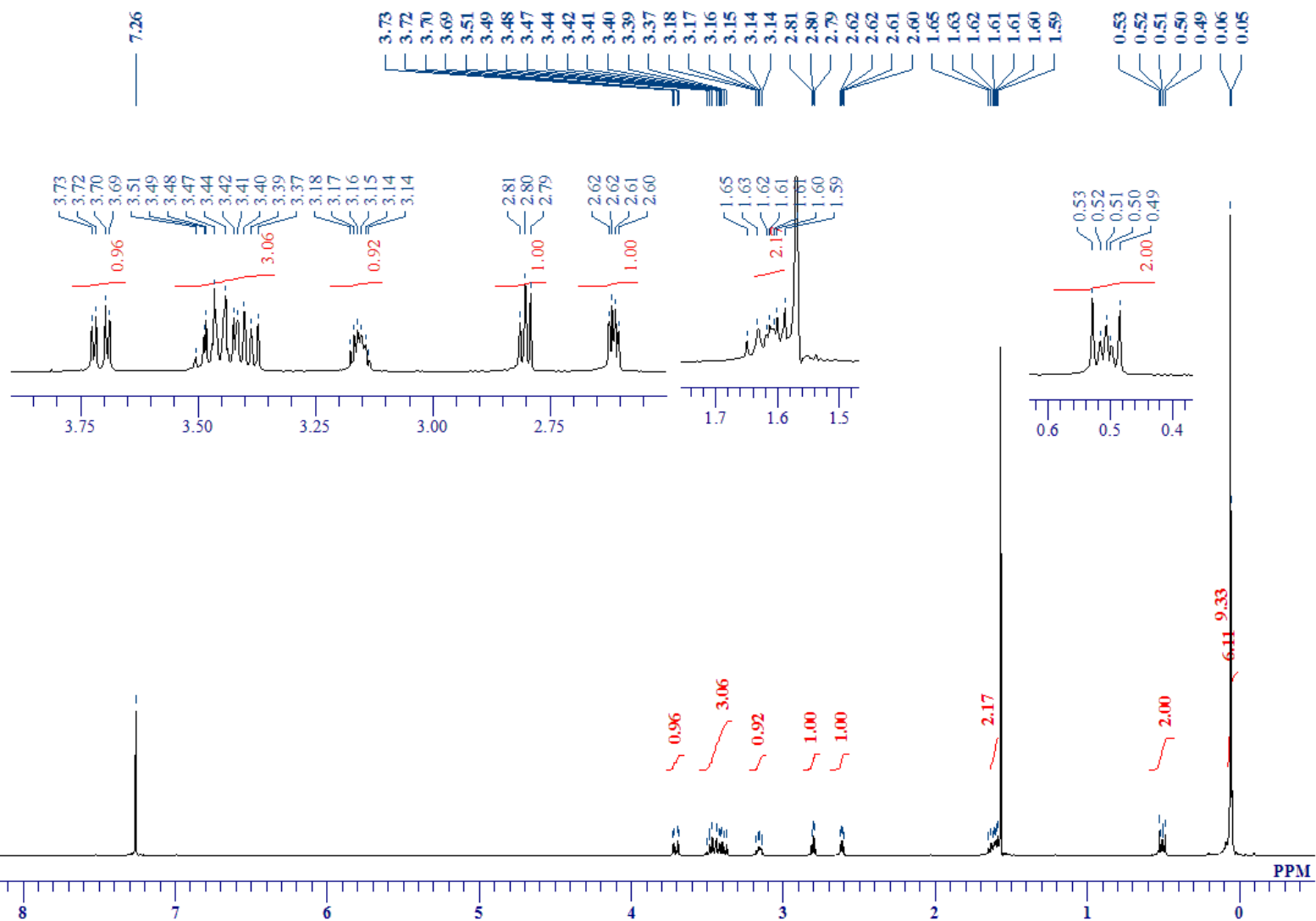
Figure $\mathrm{S} 13-2 .{ }^{13} \mathrm{C}$ NMR spectrum of a solution of $9 \mathrm{a}$ in $\mathrm{CDCl}_{3}$ at room temperature
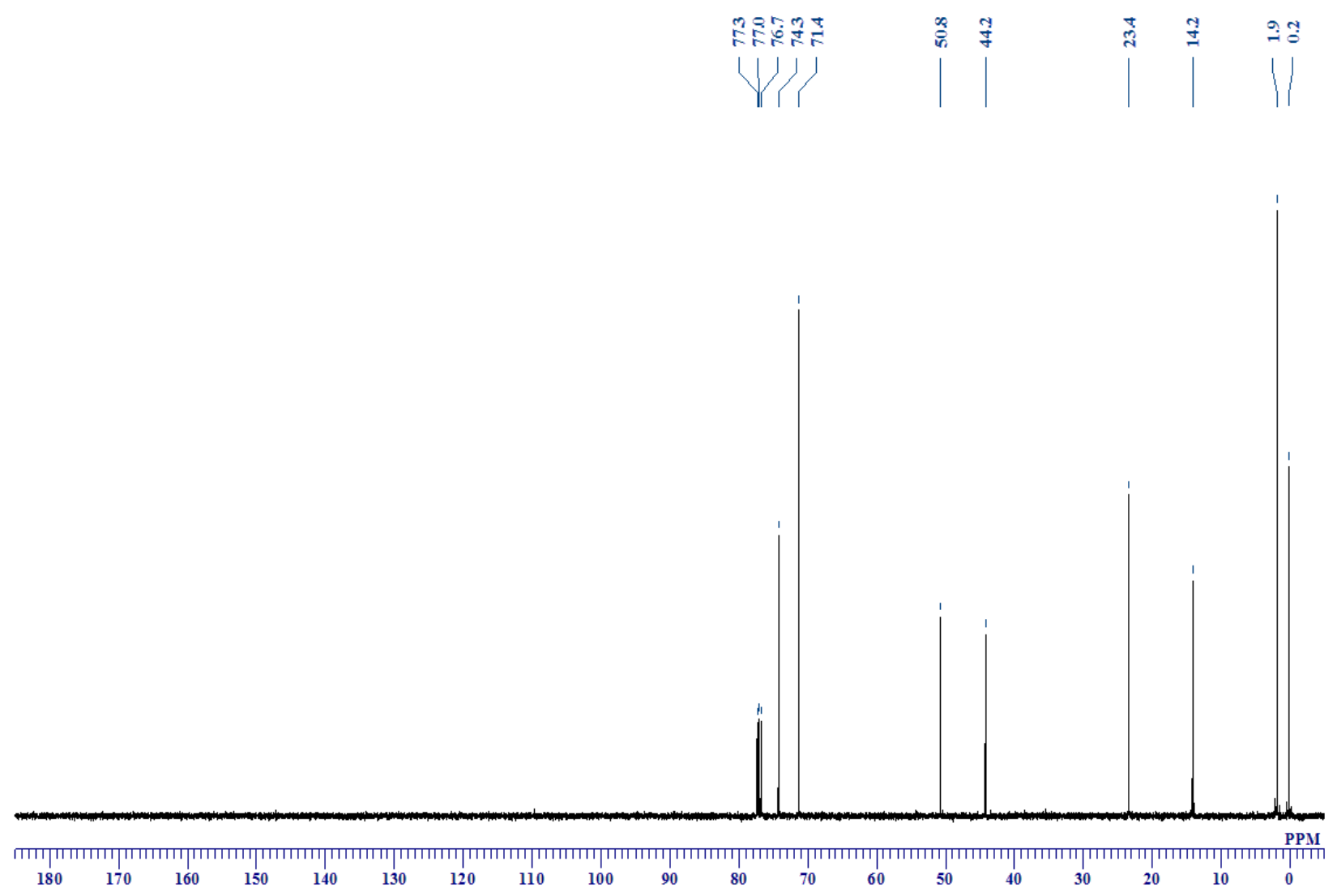

Figure S13-3. ${ }^{29} \mathrm{Si} \mathrm{NMR}$ spectrum of a solution of $9 \mathrm{a}$ in $\mathrm{CDCl}_{3}$ at room temperature

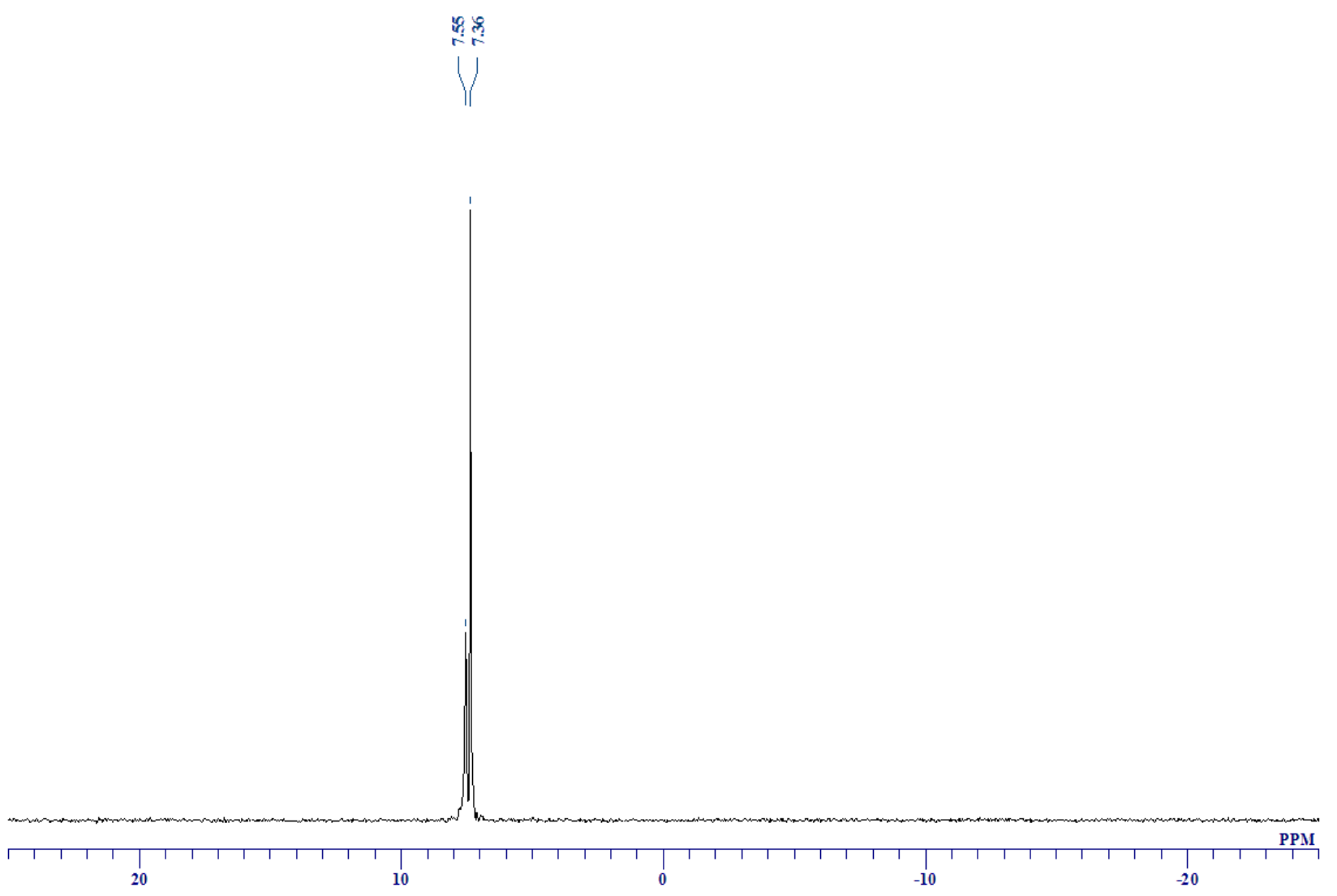


Figure $\mathrm{S} 14-1 .{ }^{1} \mathrm{H}$ NMR spectrum of a solution of $10 \mathrm{a}$ in $\mathrm{CDCl}_{3}$ at room temperature

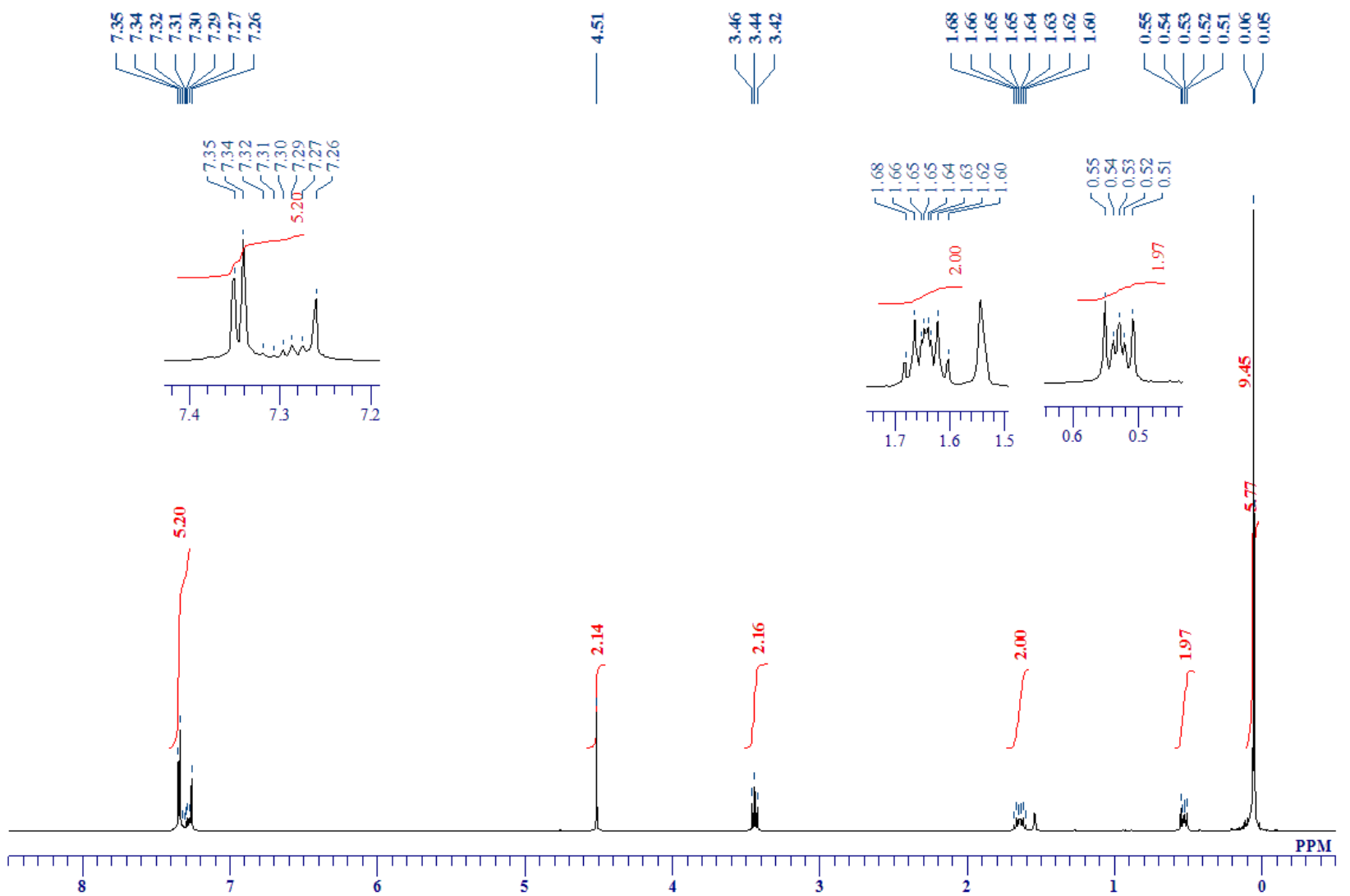

Figure $\mathrm{S} 14-2 .{ }^{13} \mathrm{C}$ NMR spectrum of a solution of $10 \mathrm{a}$ in $\mathrm{CDCl}_{3}$ at room temperature

||

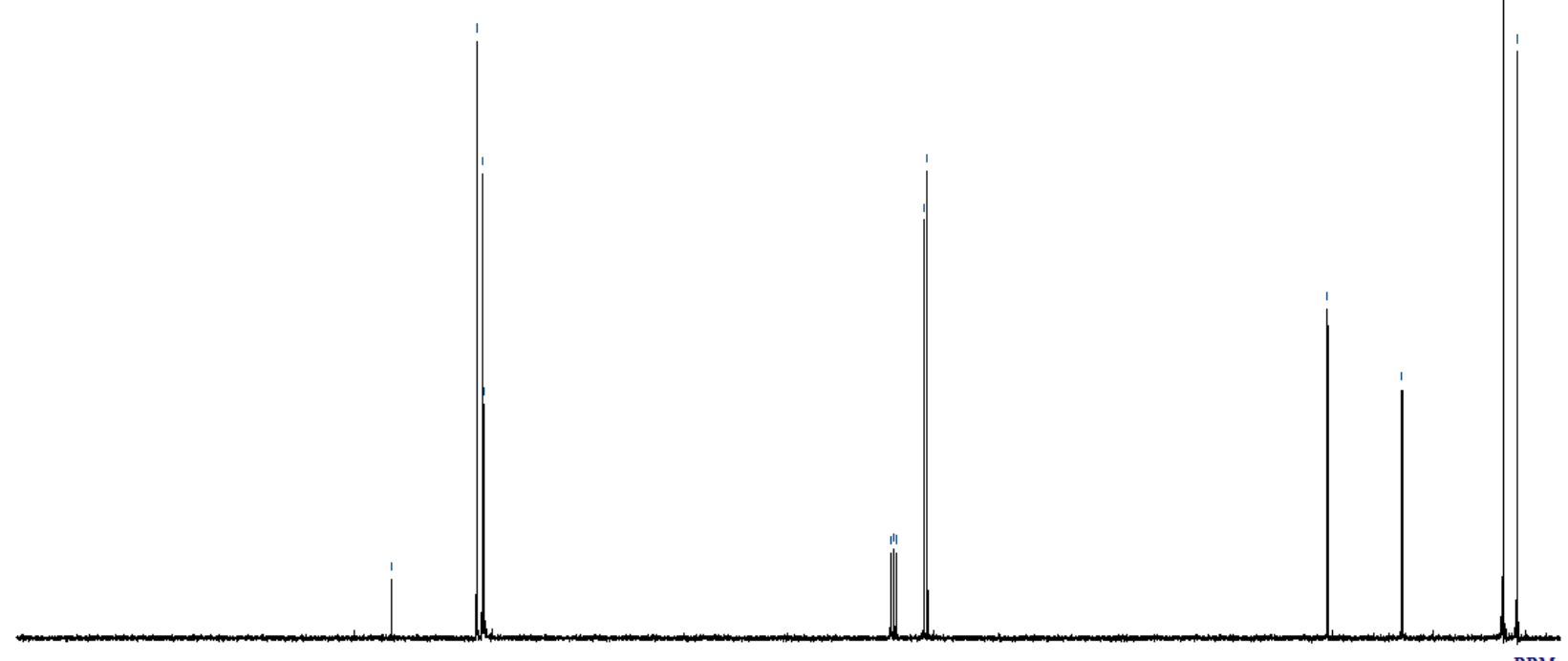

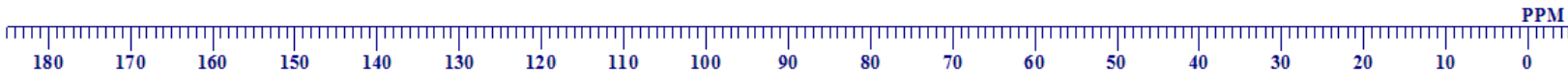
180
$170 \quad 160$
150

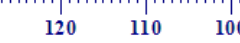


Figure S14-3. ${ }^{29} \mathrm{Si}$ NMR spectrum of solution of $10 \mathrm{a}$ in $\mathrm{CDCl}_{3}$ at room temperature

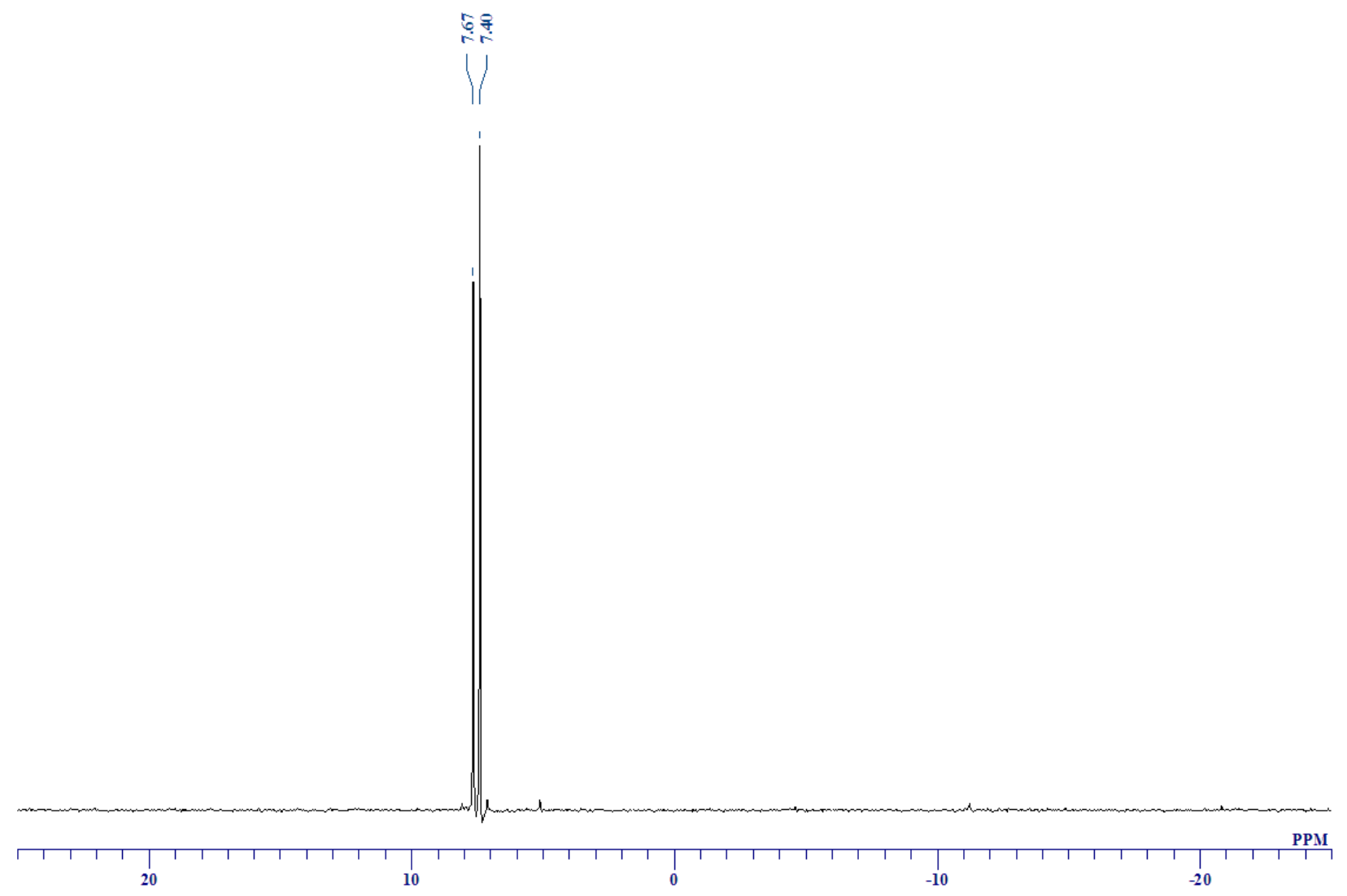

Figure $\mathrm{S} 15-1 .{ }^{1} \mathrm{H}$ NMR spectrum of a solution of $11 \mathrm{a}$ in $\mathrm{CDCl}_{3}$ at room temperature

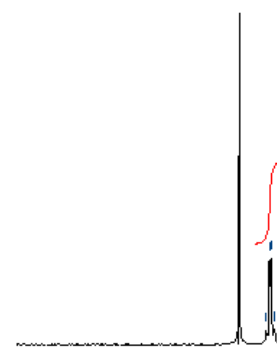

$\operatorname{r|T|ग~}$
7898

i)
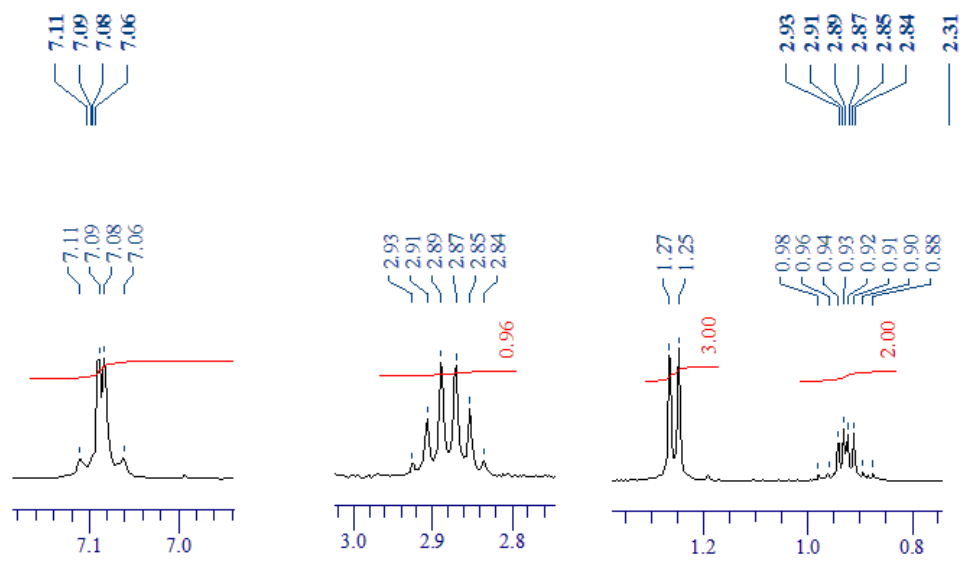

1.11

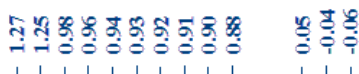

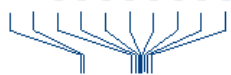

$1 /$
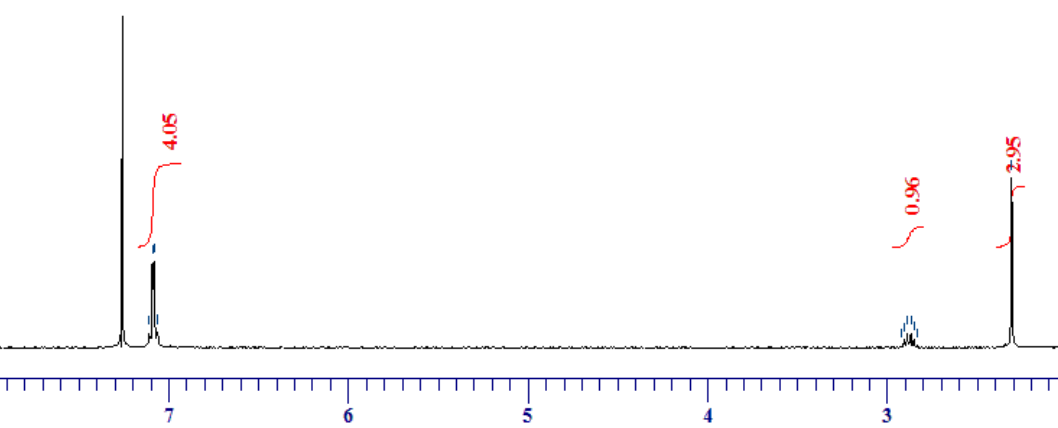
Figure S15-2. ${ }^{13} \mathrm{C}$ NMR spectrum of a solution of $11 \mathrm{a}$ in $\mathrm{CDCl}_{3}$ at room temperature
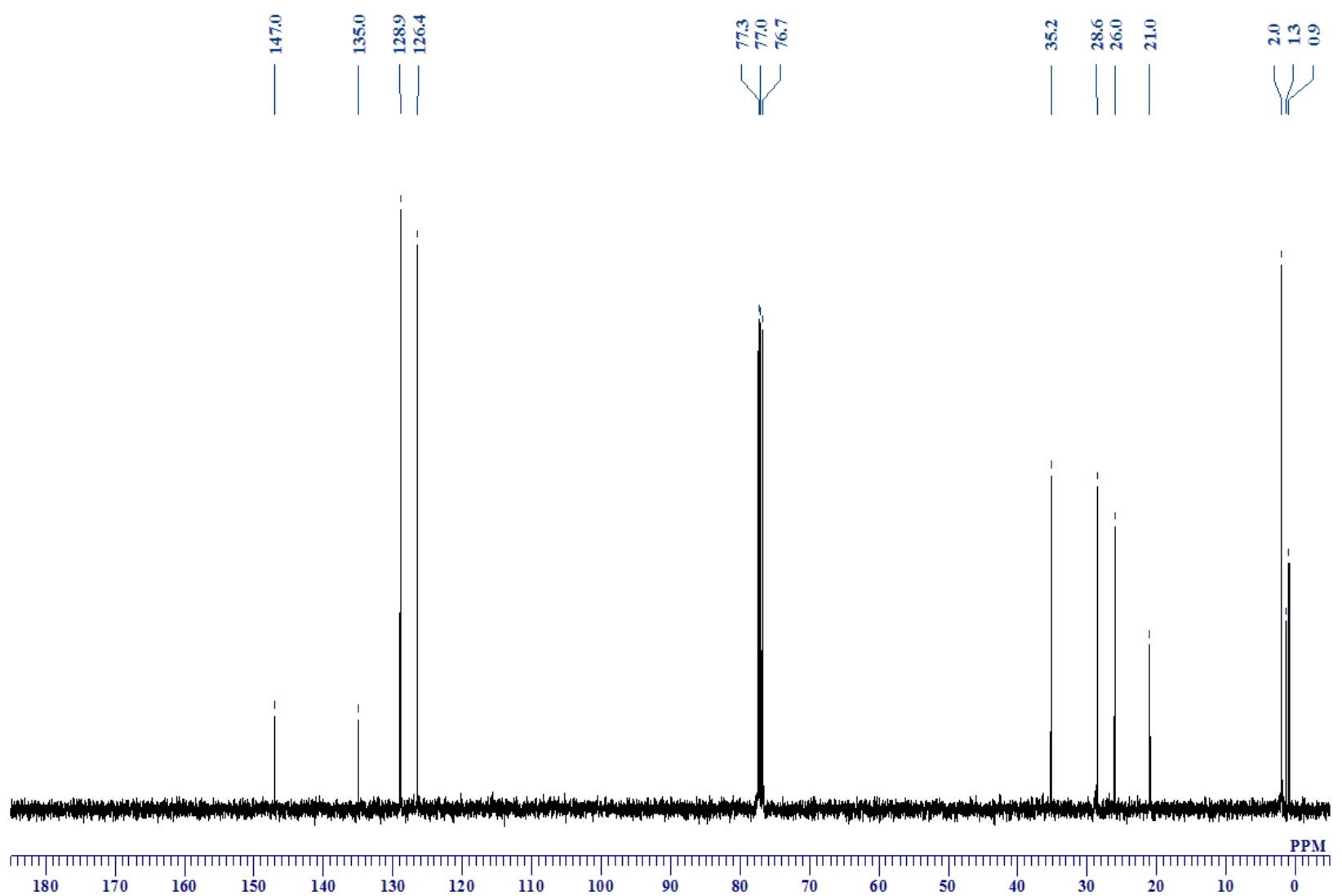

Figure S15-3. ${ }^{29} \mathrm{Si} \mathrm{NMR}$ spectrum of a solution of $11 \mathrm{a}$ in $\mathrm{CDCl}_{3}$ at room temperature

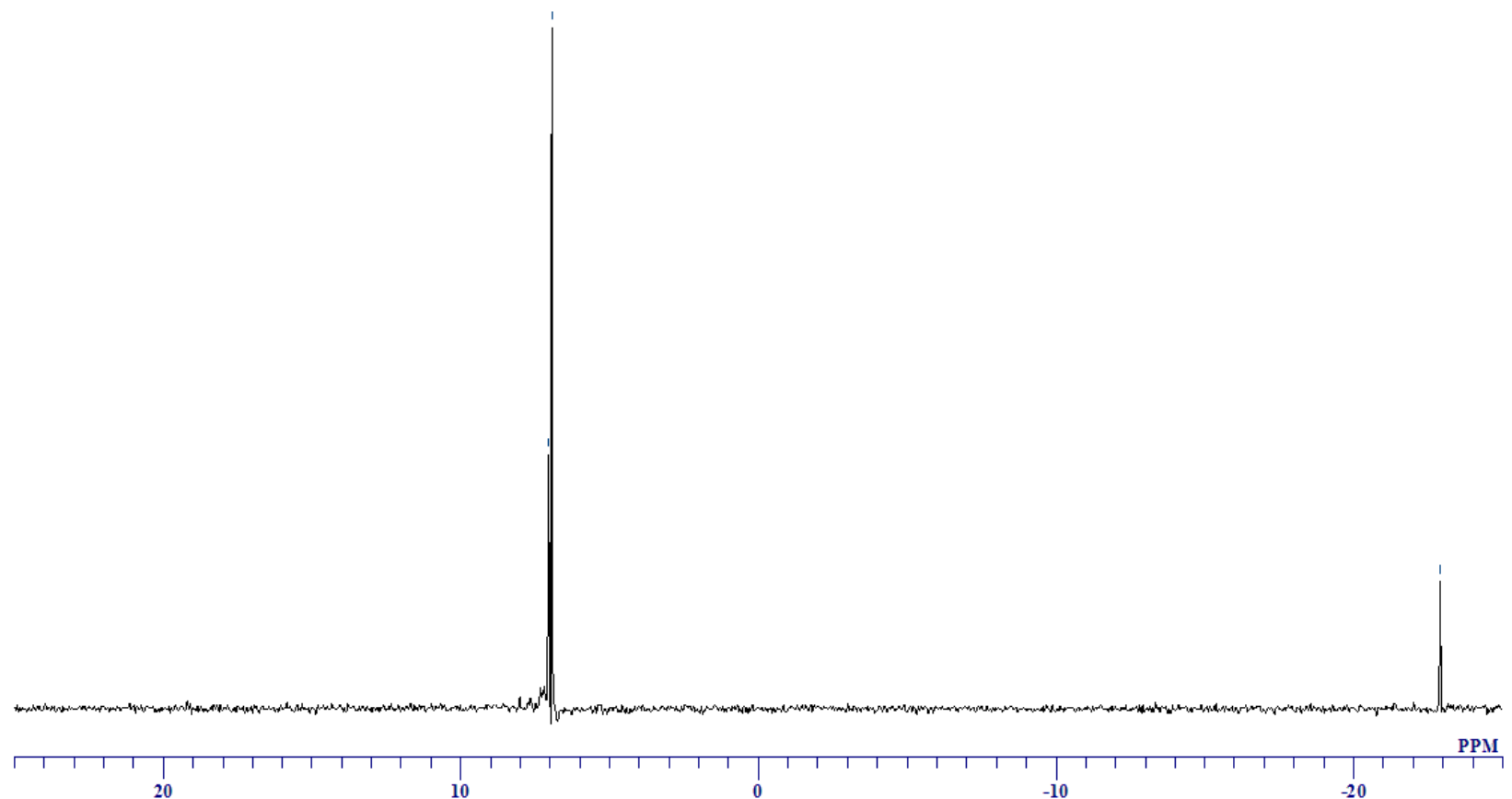


Figure $\mathrm{S} 16-1 .{ }^{1} \mathrm{H}$ NMR spectrum of a solution of $12 \mathrm{a}$ in $\mathrm{CDCl}_{3}$ at room temperature
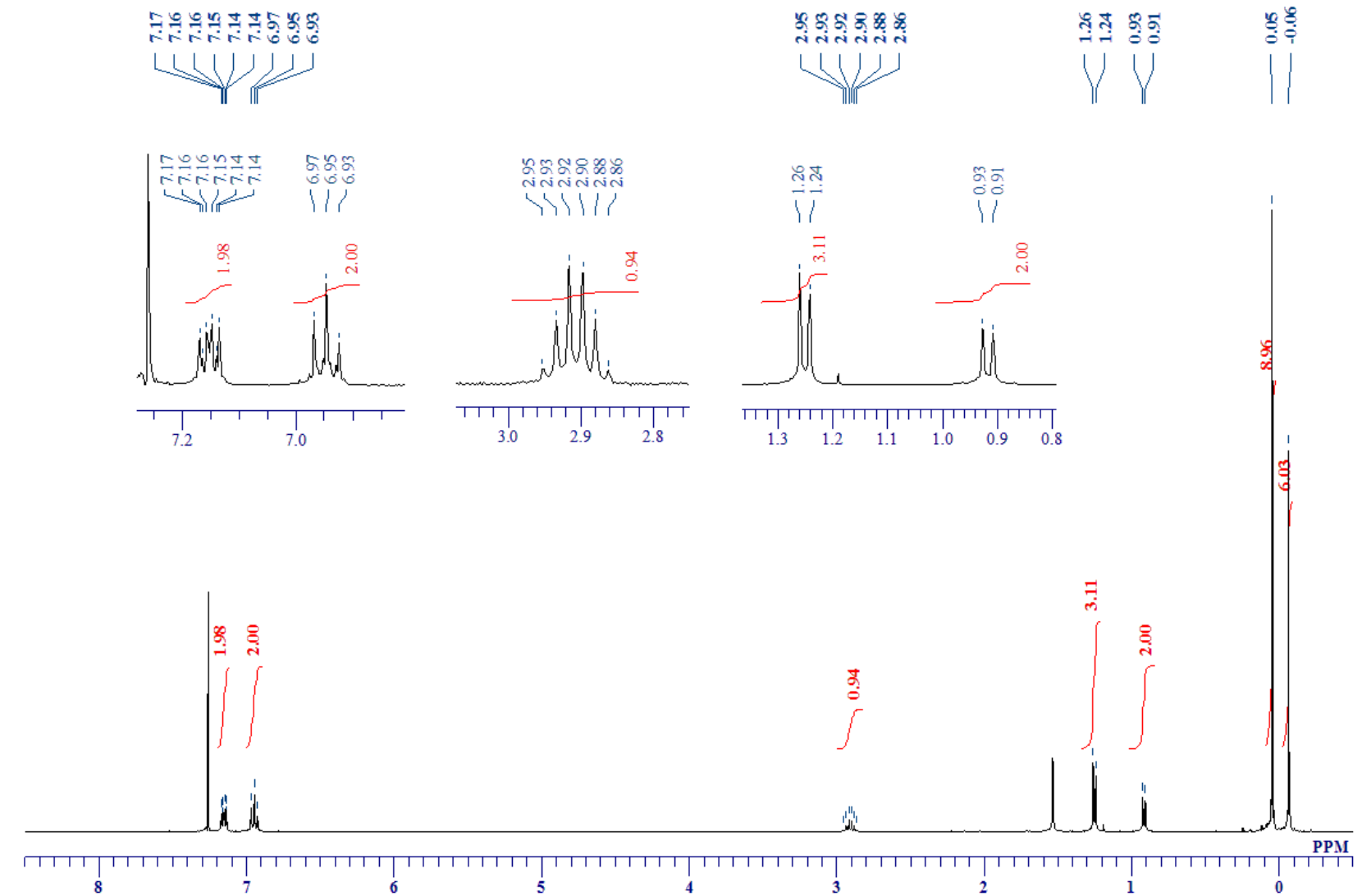

ใุํำ

sू.
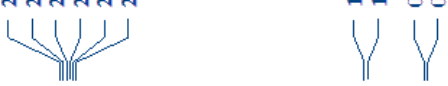

$\stackrel{8}{8}$

1.

Figure $\mathrm{S} 16-2 .{ }^{13} \mathrm{C}$ NMR spectrum of a solution of $12 \mathrm{a}$ in $\mathrm{CDCl}_{3}$ at room temperature

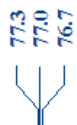

Y)

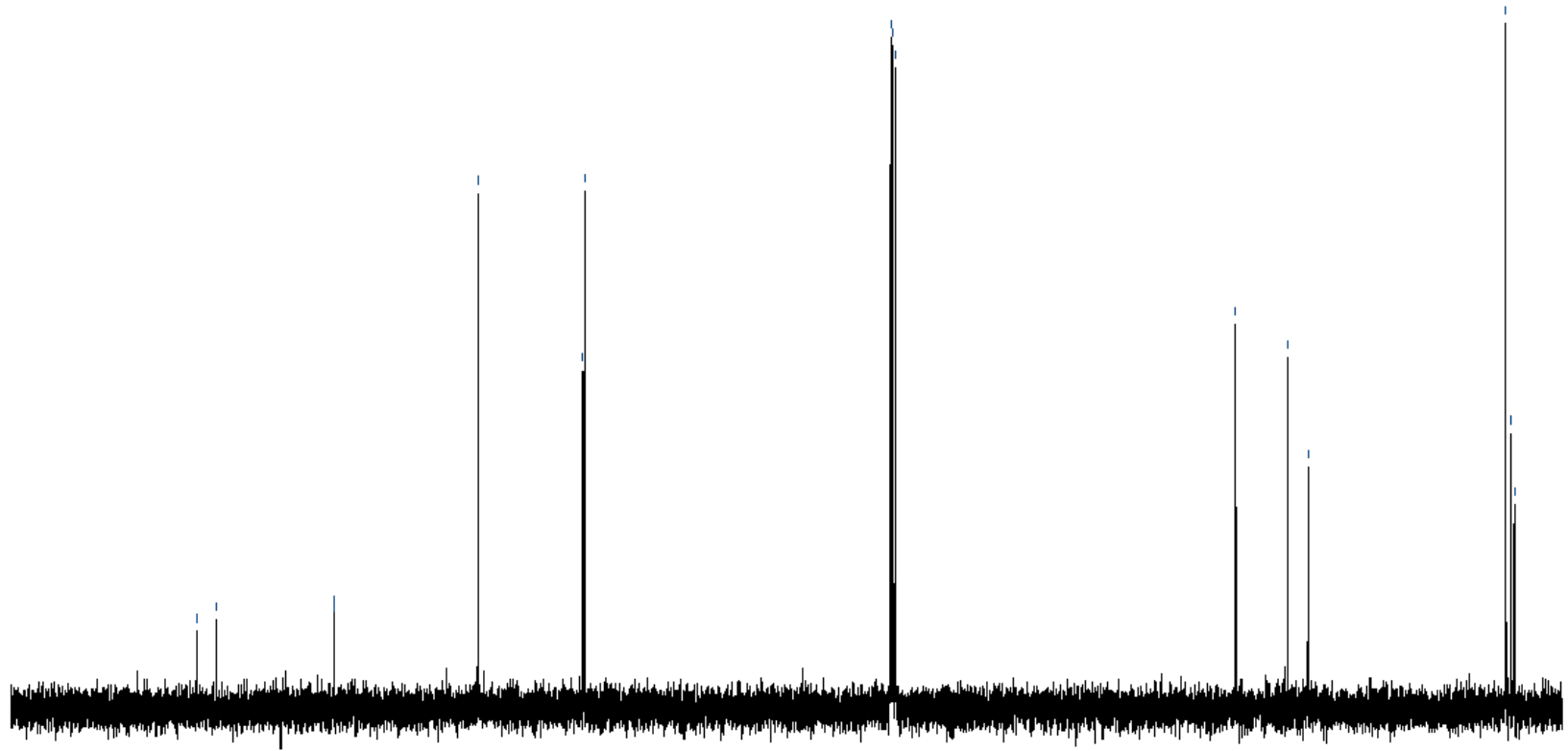

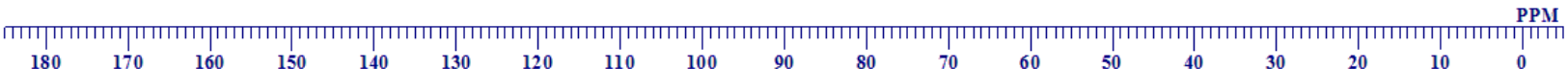


Figure $\mathrm{S} 16-3 .{ }^{19} \mathrm{~F}$ NMR spectrum of a solution of $12 \mathrm{a}$ in $\mathrm{CDCl}_{3}$ at room temperature

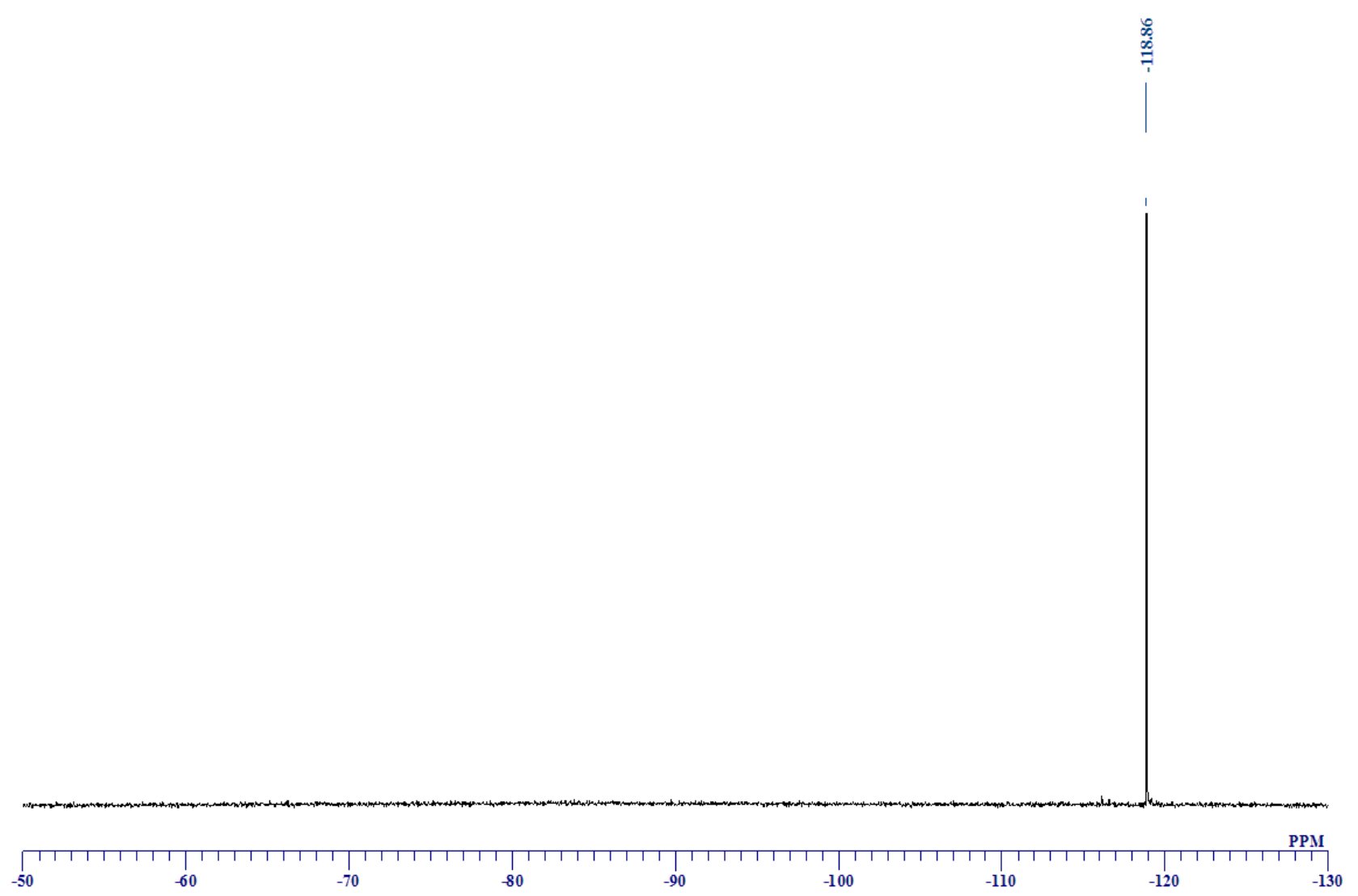

Figure S16-4. ${ }^{29} \mathrm{Si}$ NMR spectrum of a solution of $12 \mathrm{a}$ in $\mathrm{CDCl}_{3}$ at room temperature

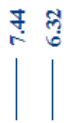

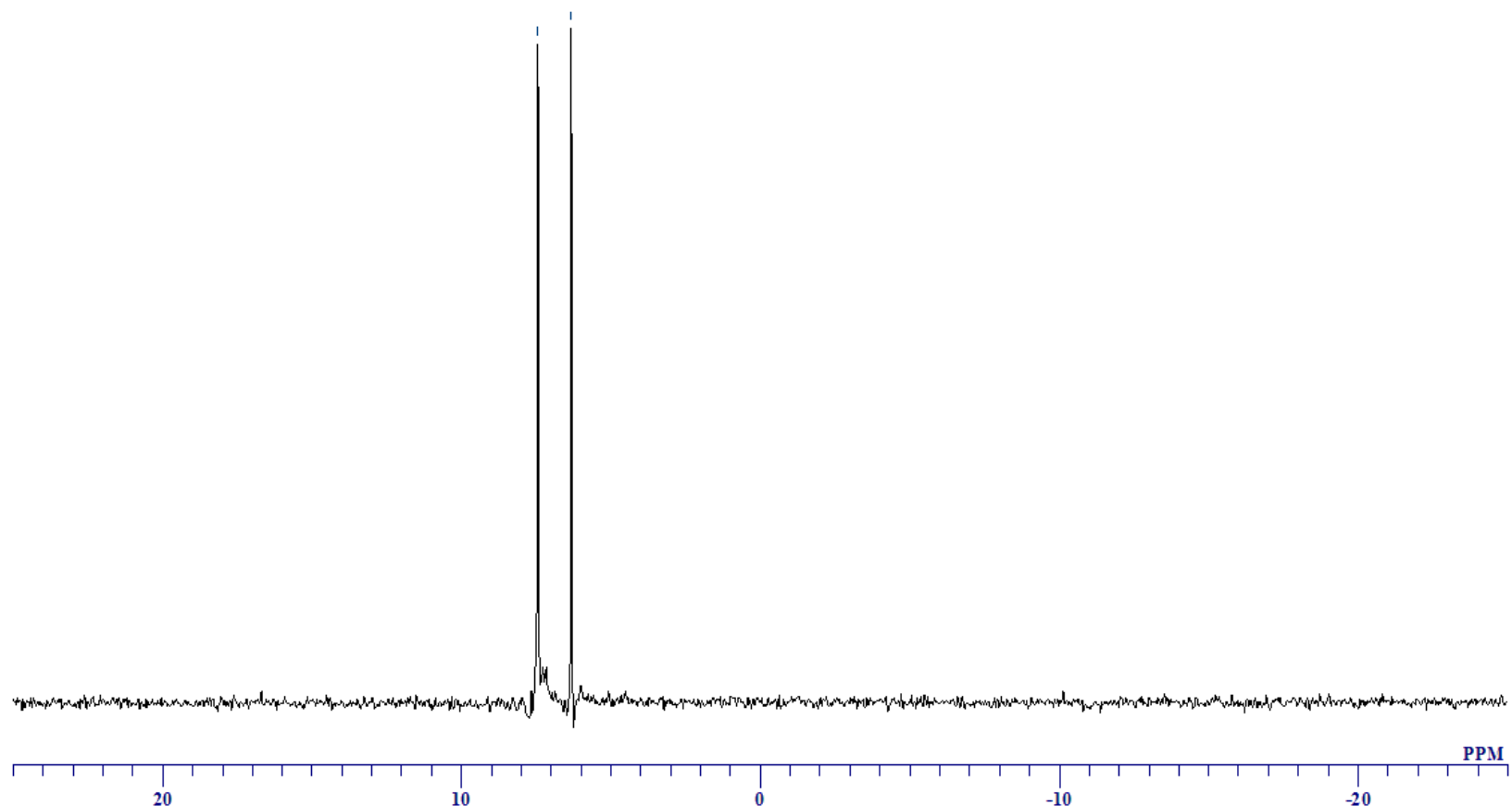


Figure $\mathrm{S} 17-1 .{ }^{1} \mathrm{H}$ NMR spectrum of a solution of $13 \mathrm{a}$ in $\mathrm{CDCl}_{3}$ at room temperature

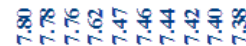
UMU
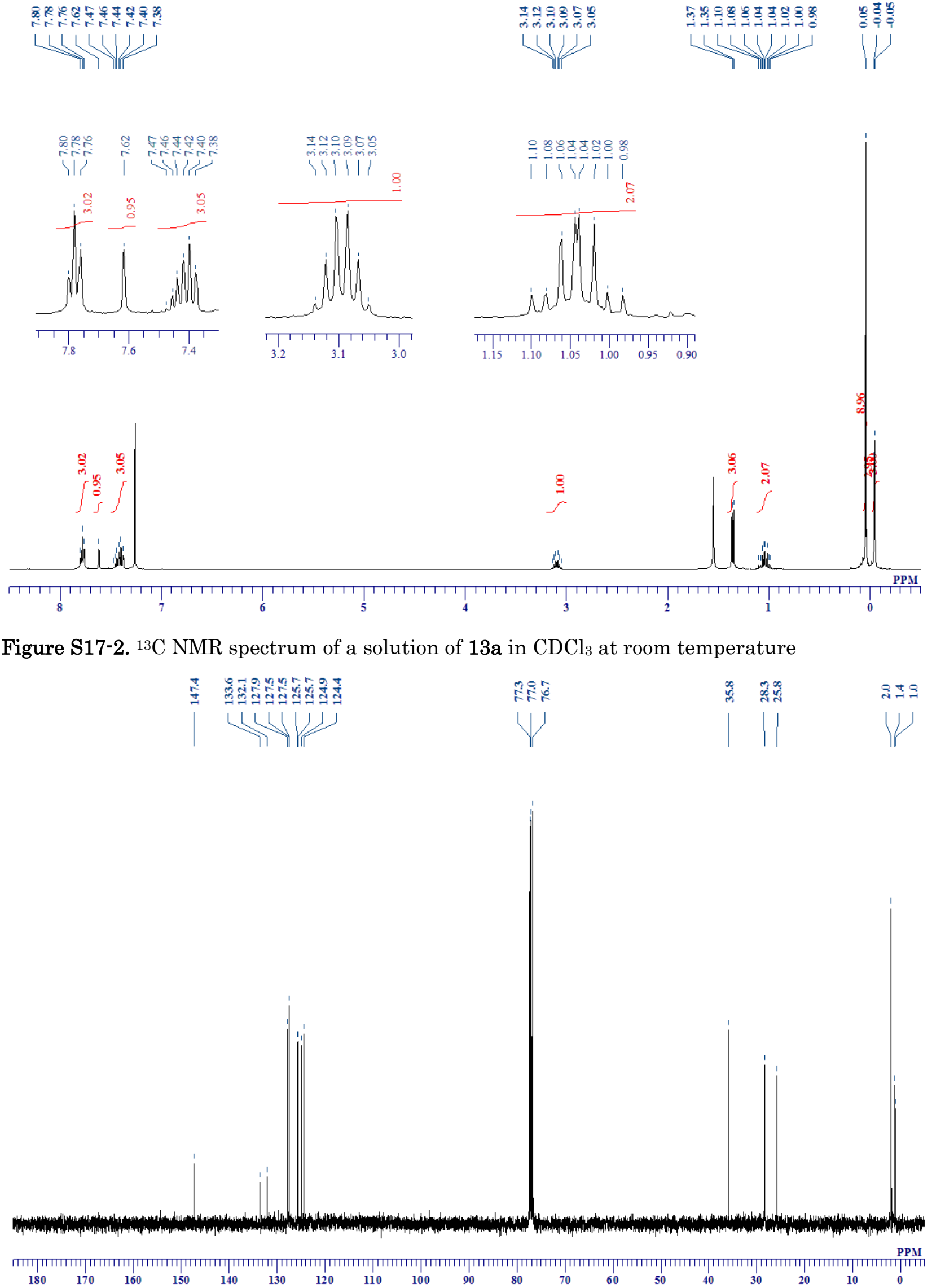
Figure S17-3. ${ }^{29} \mathrm{Si}$ NMR spectrum of a solution of $13 \mathrm{a}$ in $\mathrm{CDCl}_{3}$ at room temperature
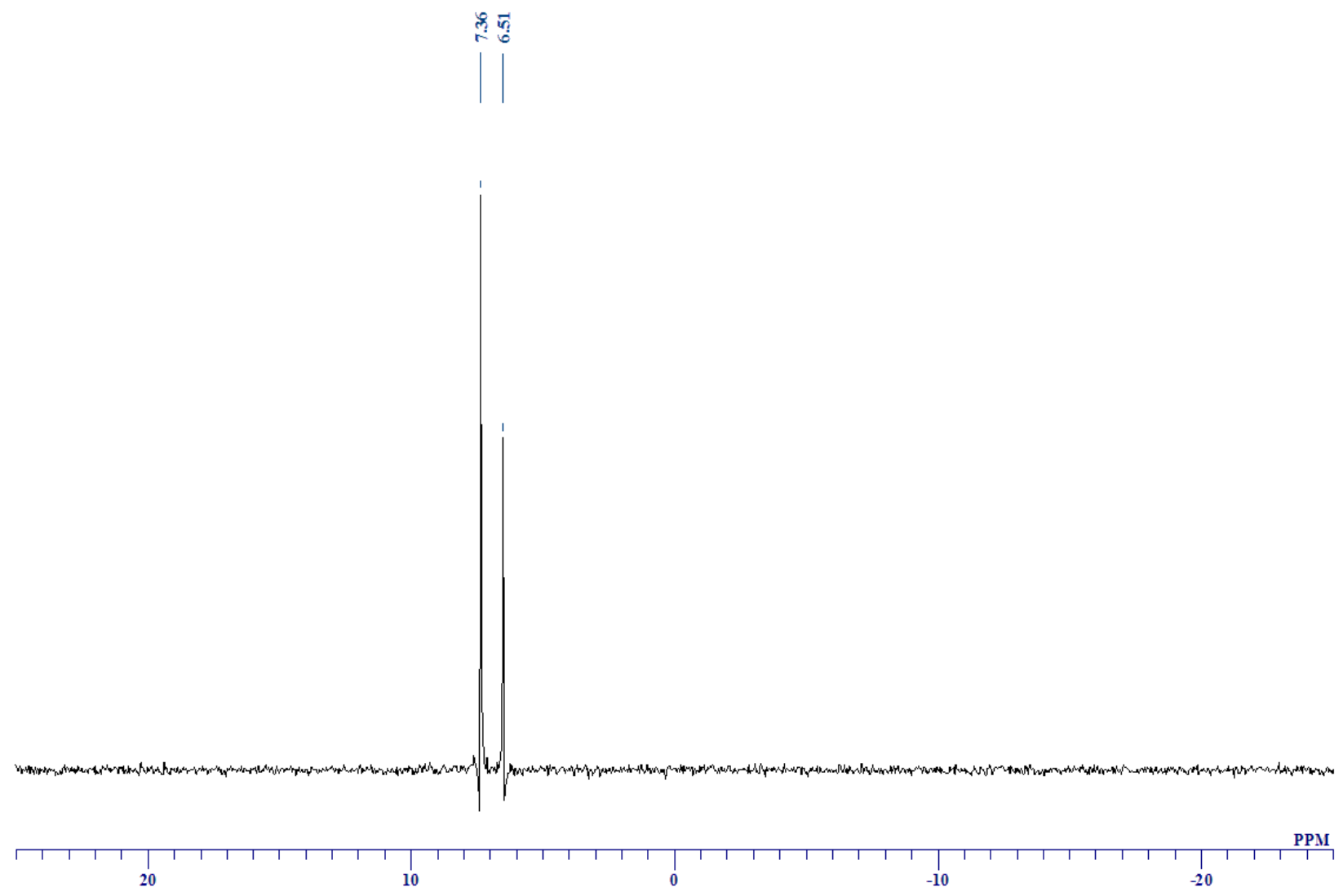

Figure S18-1. ${ }^{1} \mathrm{H}$ NMR spectrum of a solution of $14 \mathrm{a}$ in $\mathrm{CDCl}_{3}$ at room temperature
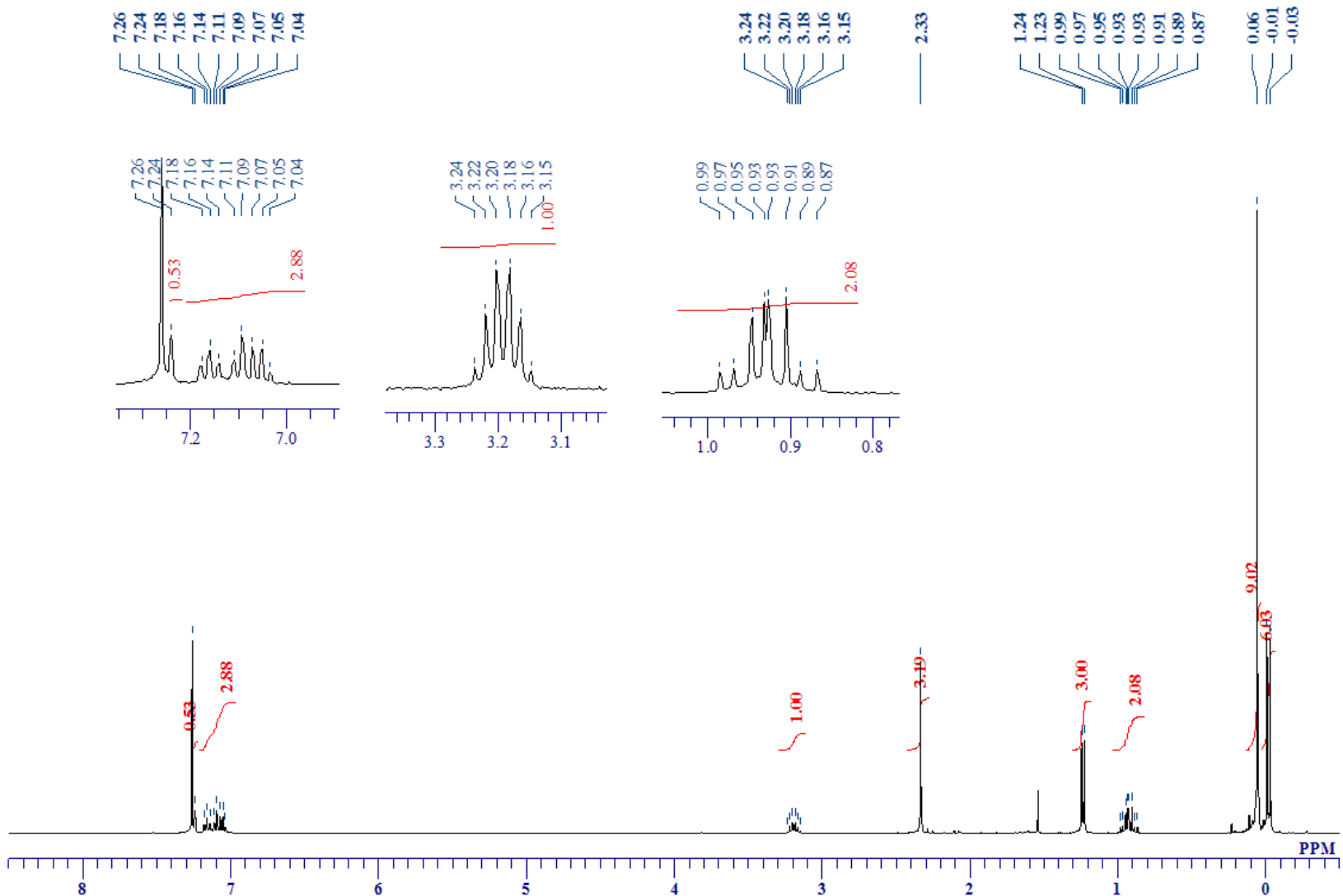
Figure S18-2. ${ }^{13} \mathrm{C}$ NMR spectrum of a solution of $14 \mathrm{a}$ in $\mathrm{CDCl}_{3}$ at room temperature
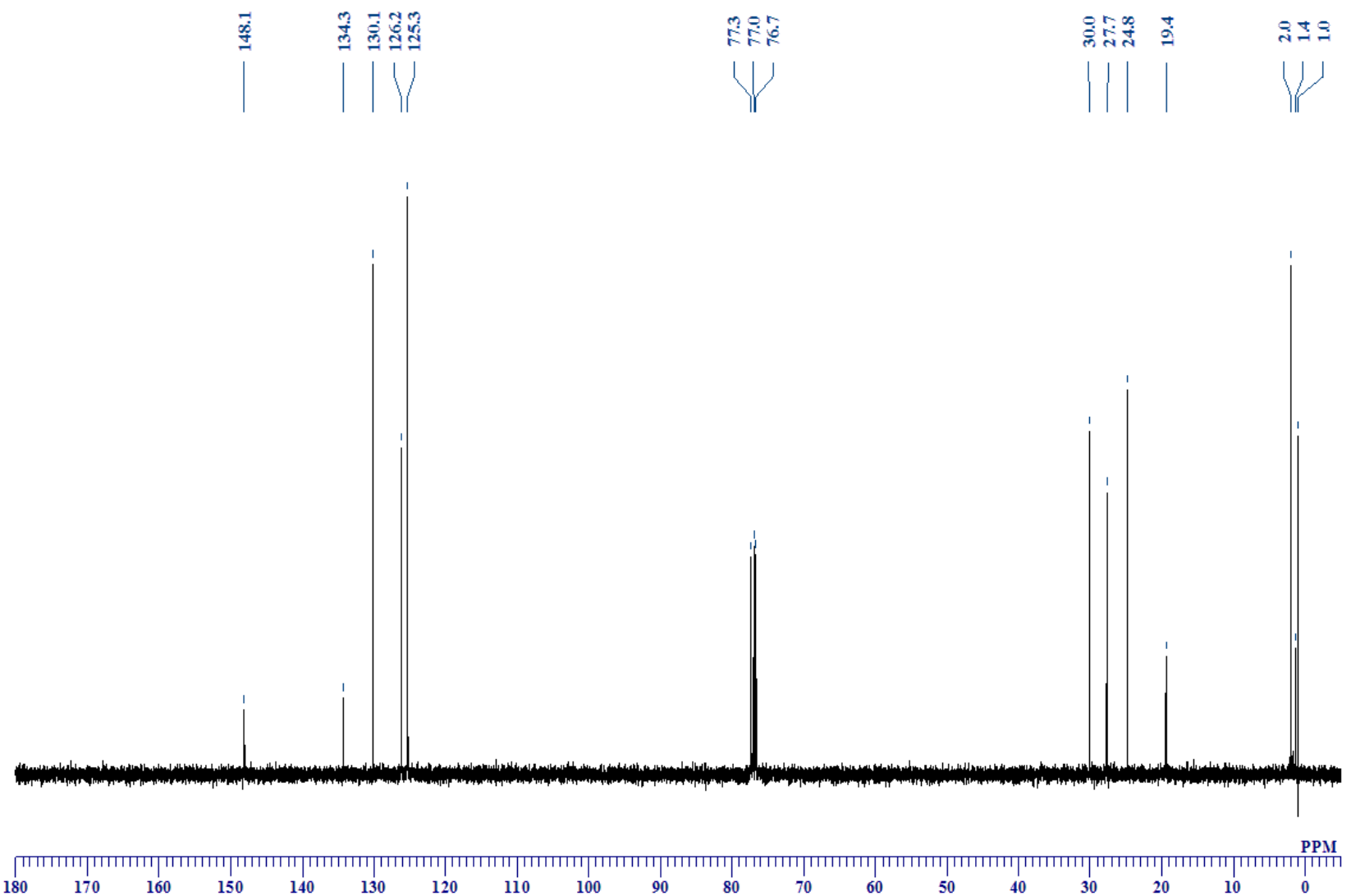

Figure S18-3. ${ }^{29} \mathrm{Si}$ NMR spectrum of a solution of $14 \mathrm{a}$ in $\mathrm{CDCl}_{3}$ at room temperature

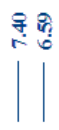

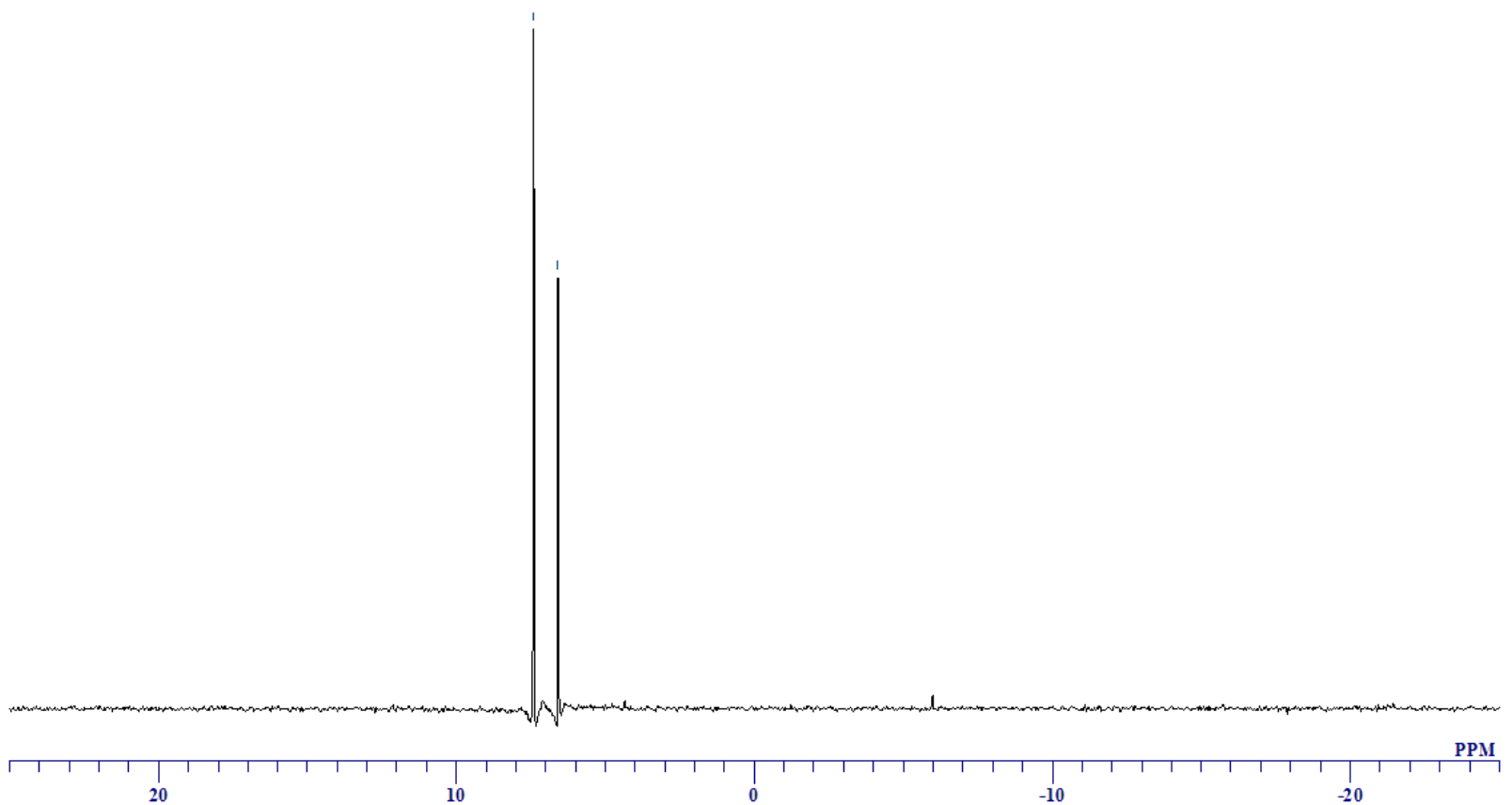


Figure S19-1. ${ }^{1} \mathrm{H}$ NMR spectrum of a solution of $15 \mathrm{a}$ in $\mathrm{CDCl}_{3}$ at room temperature

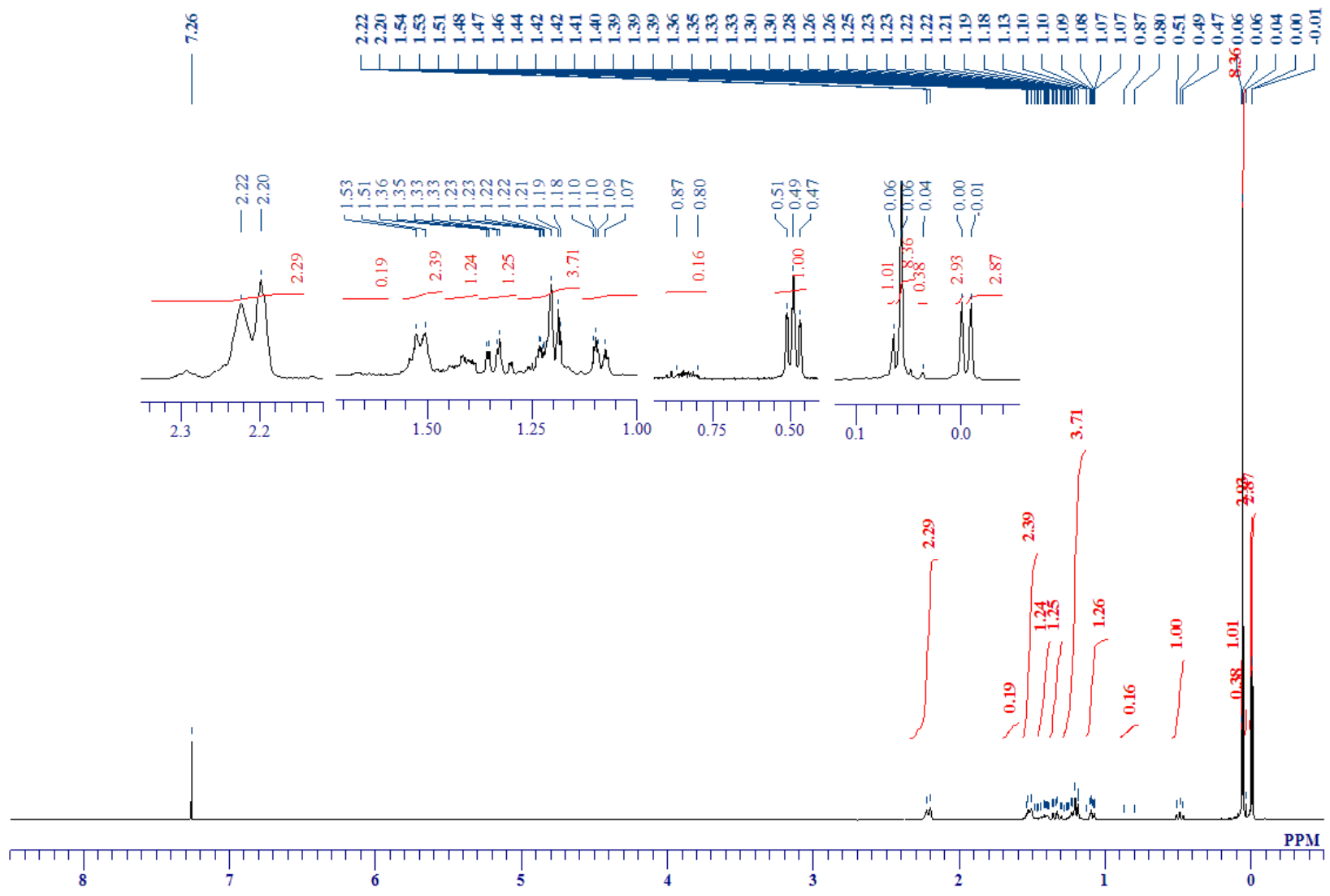

Figure S19-2. ${ }^{13} \mathrm{C}$ NMR spectrum of a solution of $15 \mathrm{a}$ in $\mathrm{CDCl}_{3}$ at room temperature

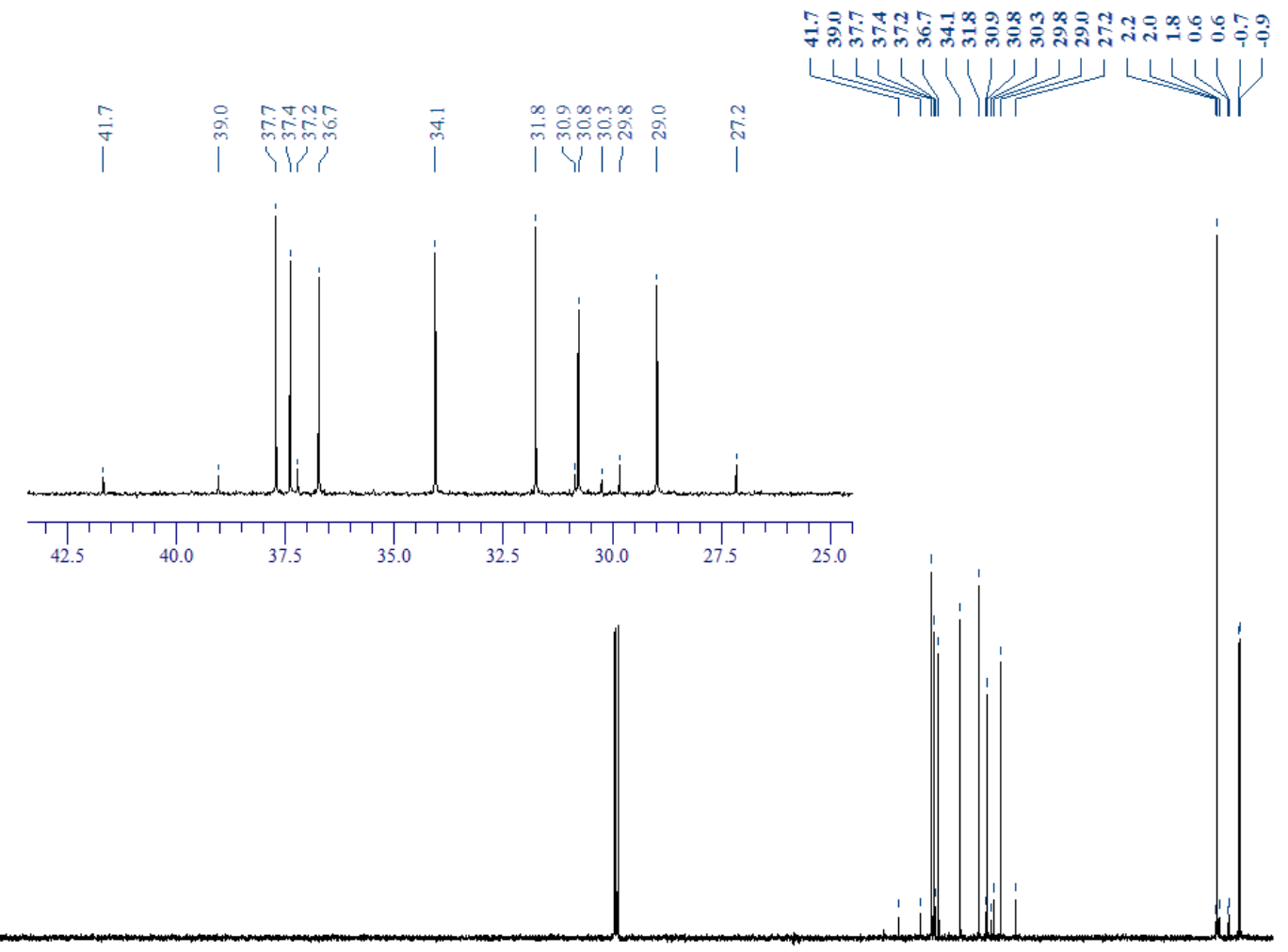

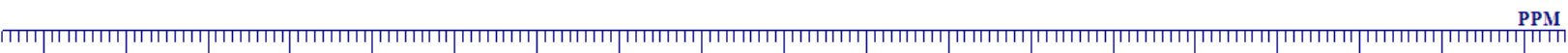

$\begin{array}{lllllllllllllllllll}180 & 170 & 160 & 150 & 140 & 130 & 120 & 110 & 100 & 90 & 80 & 70 & 60 & 50 & 40 & 30 & 20 & 10 & 0\end{array}$ 
Figure S19-3. ${ }^{29} \mathrm{Si} \mathrm{NMR}$ spectrum of a solution of $15 \mathrm{a}$ in $\mathrm{CDCl}_{3}$ at room temperature M.

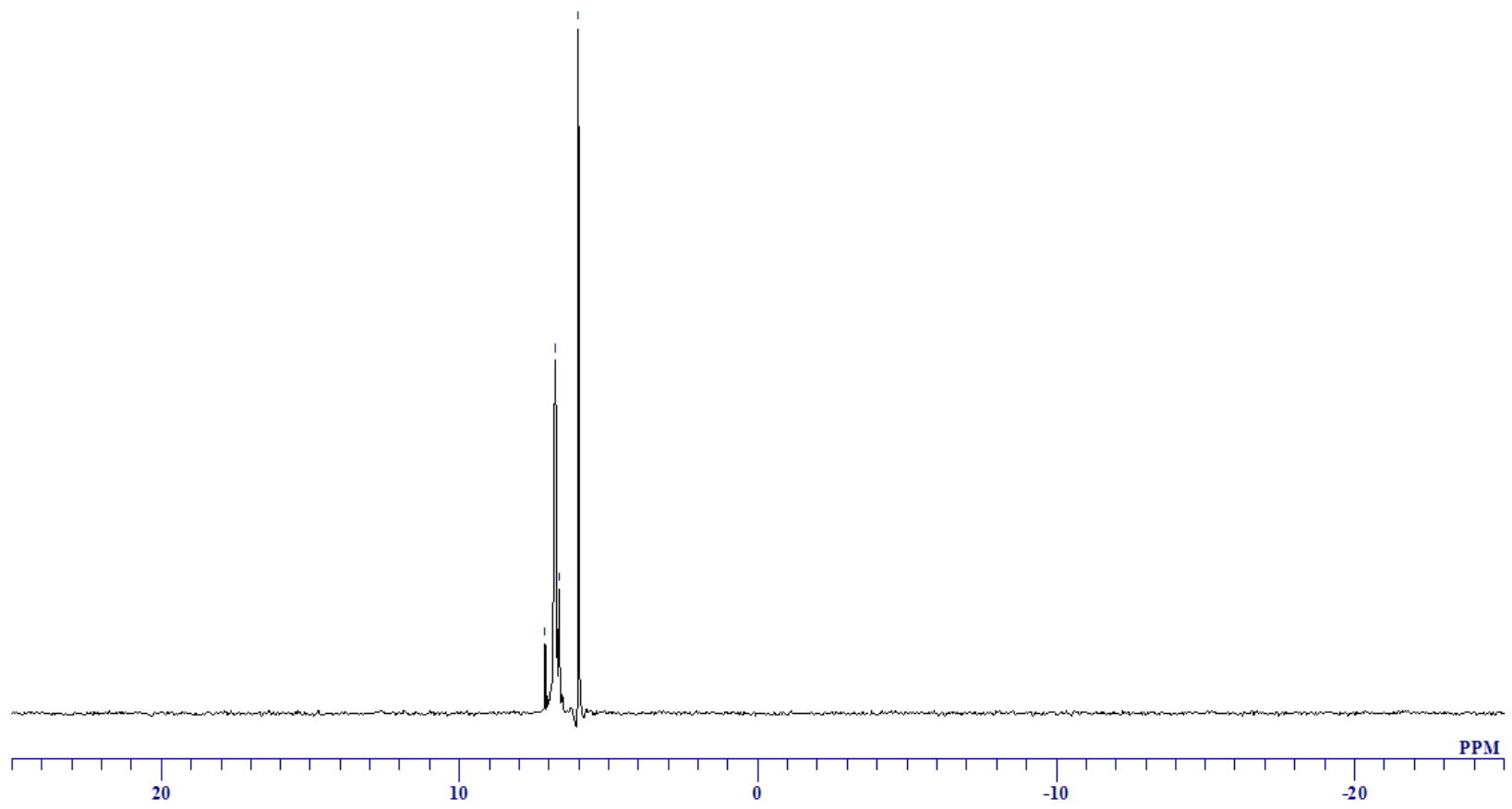

Figure S19-4. ${ }^{1} \mathrm{H}^{-1} \mathrm{H}$ cosy NMR spectrum of a solution of 15 a in $\mathrm{CDCl}_{3}$ at room temperature

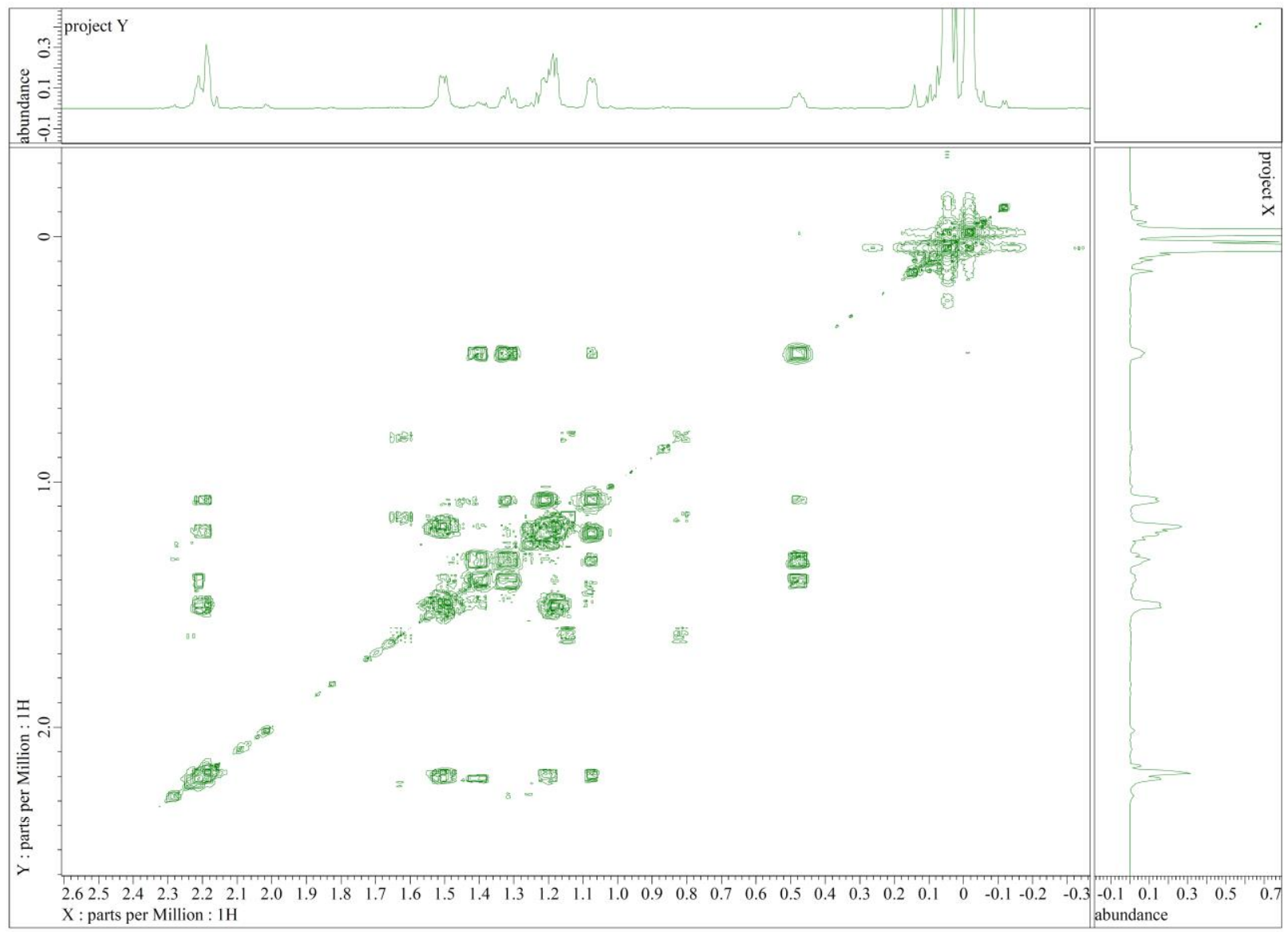


Figure $\mathbf{S} 19-5 .{ }^{1} \mathrm{H}$ noe NMR spectrum of a solution of $15 \mathrm{a}$ in $\mathrm{CDCl}_{3}$ at room temperature

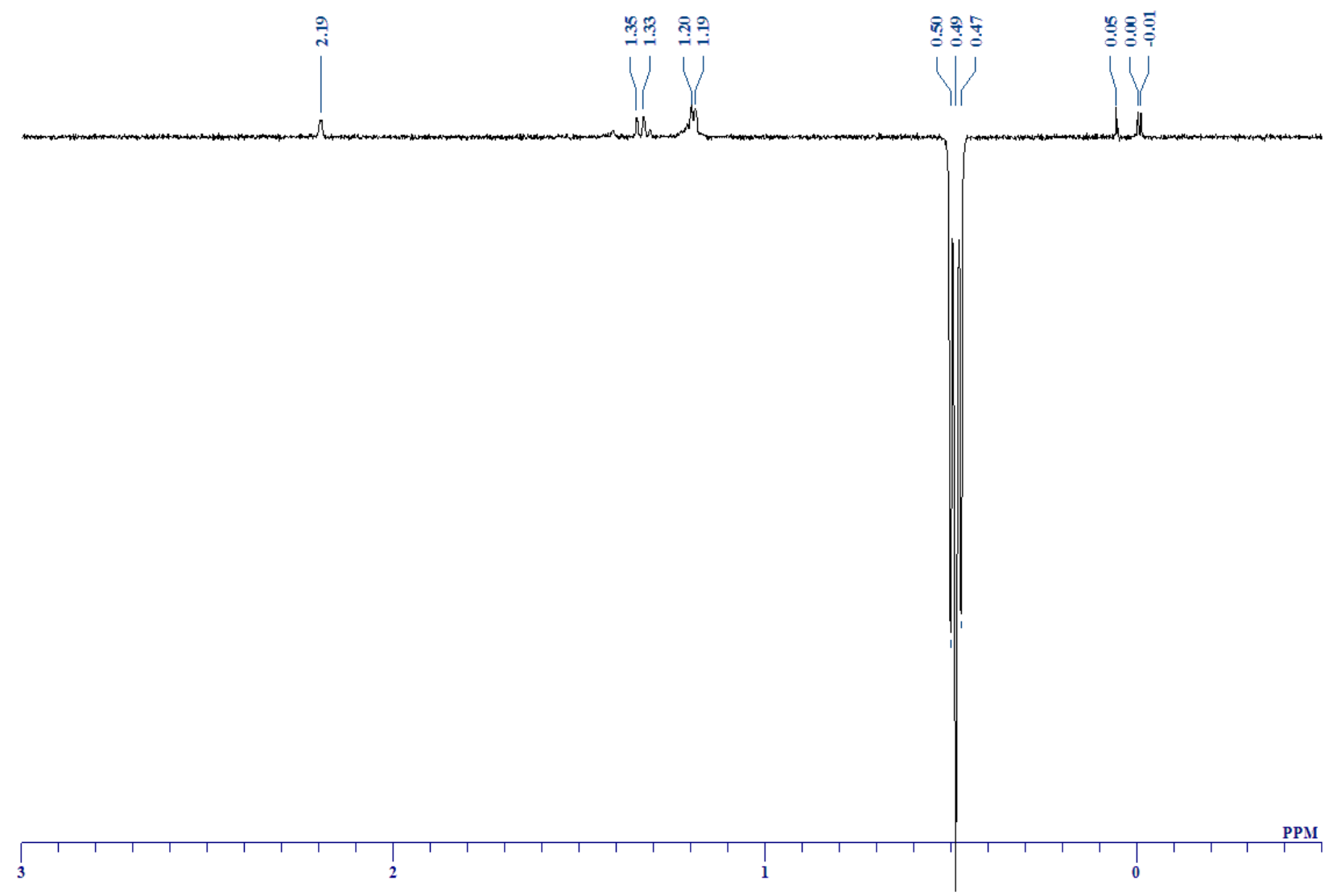

Figure $\mathrm{S} 20-1 .{ }^{1} \mathrm{H}$ NMR spectrum of a solution of $16 \mathrm{a}$ in $\mathrm{CDCl}_{3}$ at room temperature

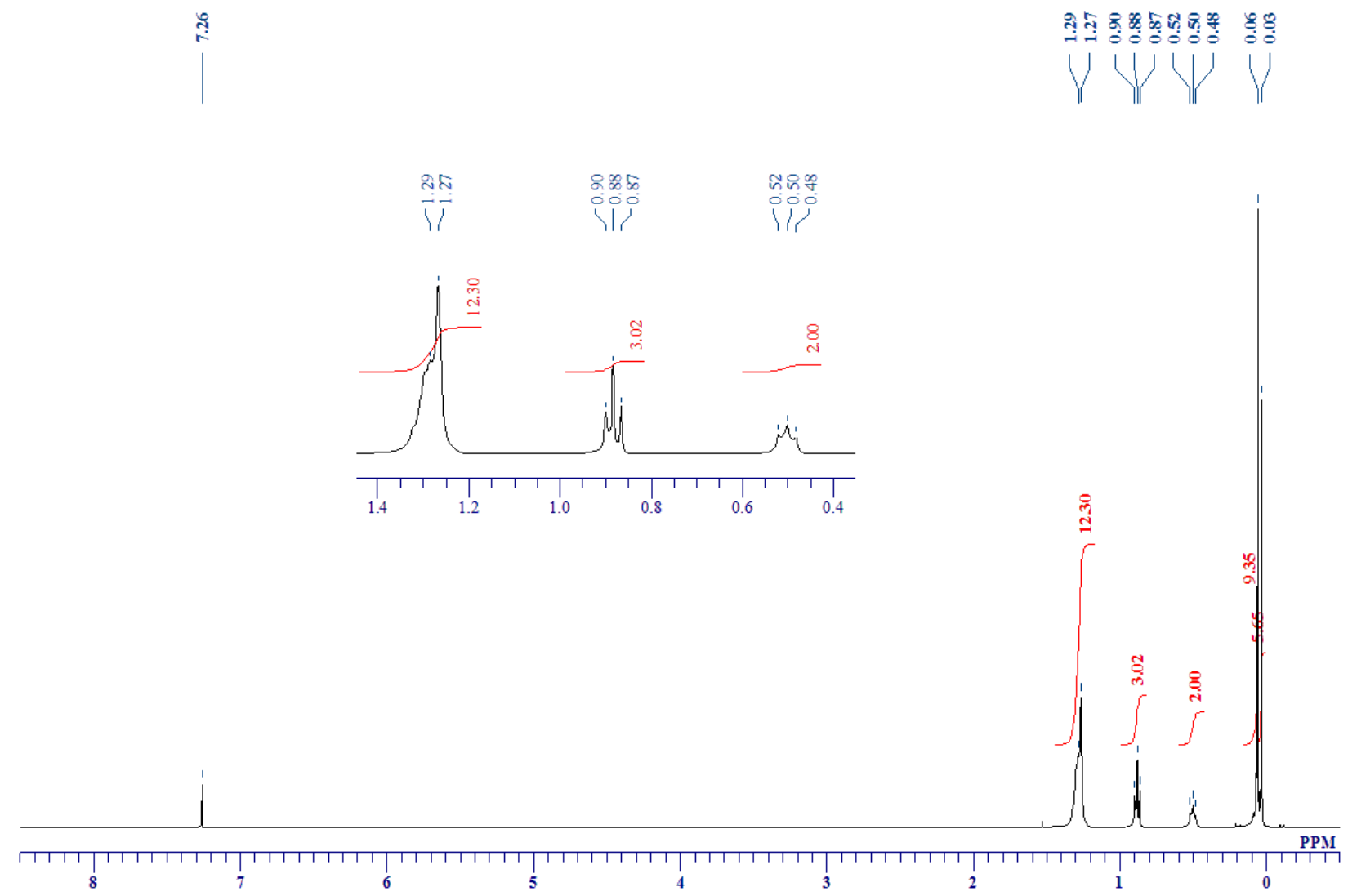


Figure $\mathrm{S} 20-2 . \quad{ }^{13} \mathrm{C} \mathrm{NMR} \mathrm{spectrum} \mathrm{of} \mathrm{a} \mathrm{solution} \mathrm{of} 16 \mathrm{a}$ in $\mathrm{CDCl}_{3}$ at room temperature
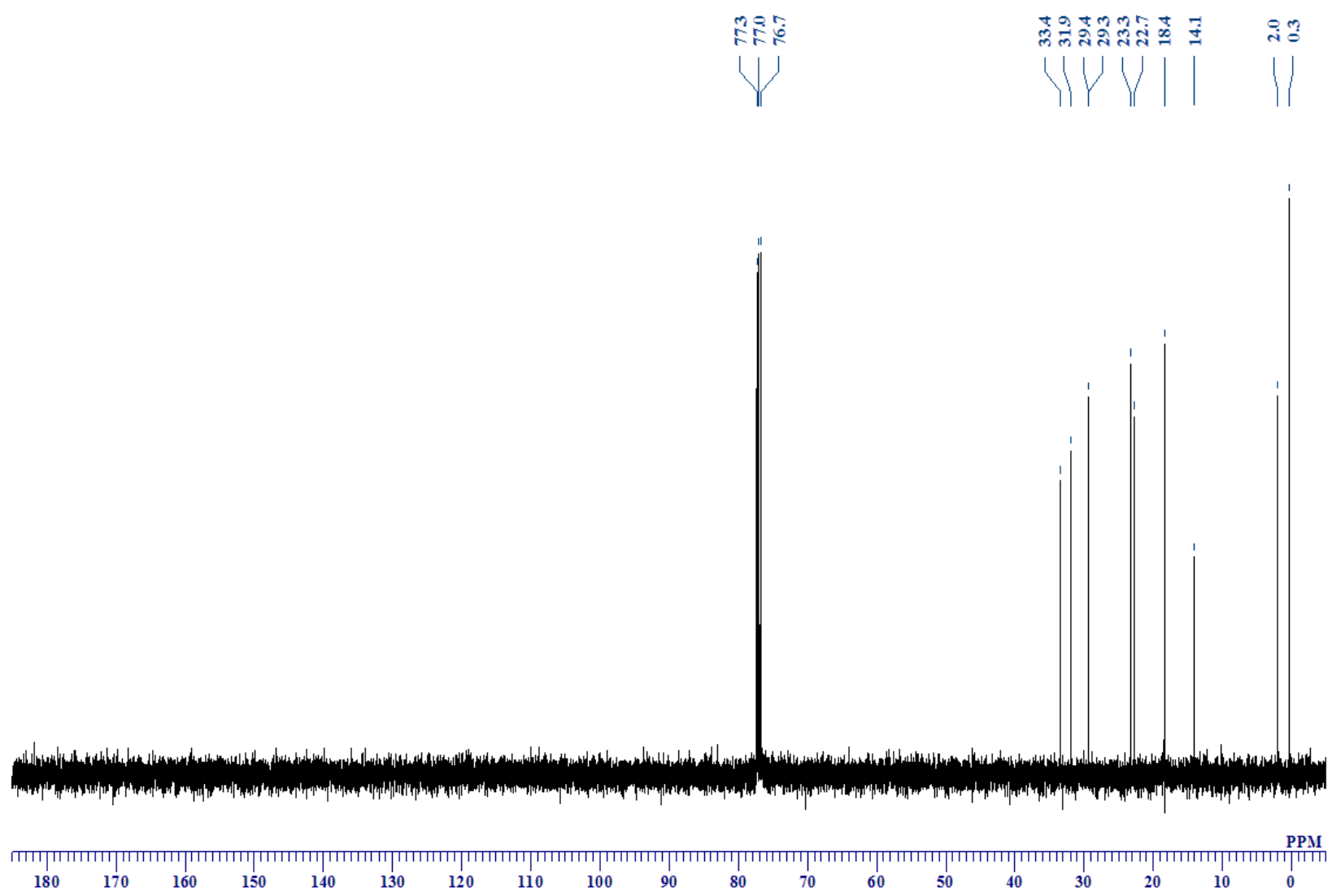

Figure S20-3. ${ }^{29} \mathrm{Si} \mathrm{NMR}$ spectrum of a solution of $\mathbf{1 6 a}$ in $\mathrm{CDCl}_{3}$ at room temperature

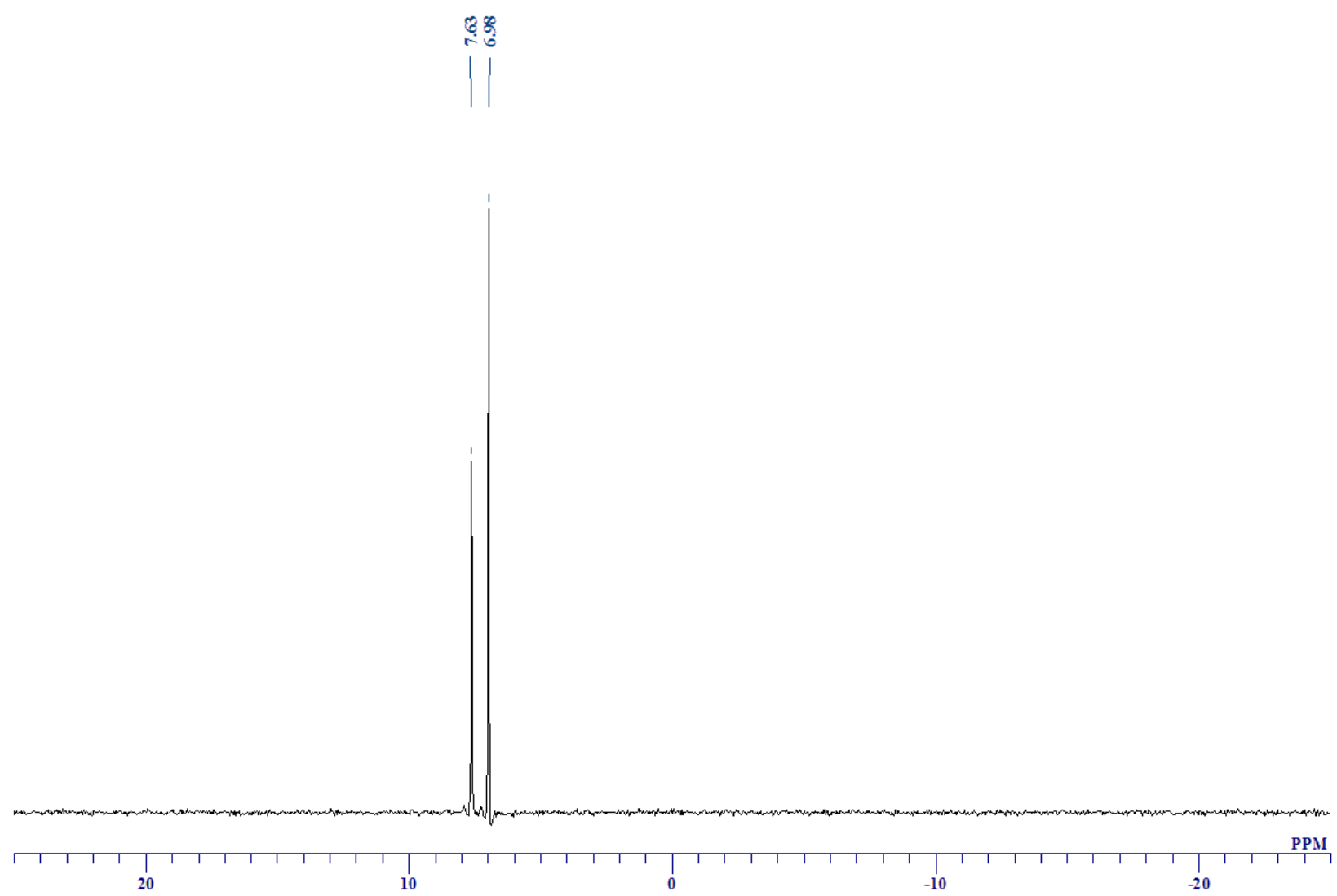


Figure $\mathrm{S} 21-1 .{ }^{1} \mathrm{H}$ NMR spectrum of a solution of $17 \mathrm{a}$ in $\mathrm{CDCl}_{3}$ at room temperature

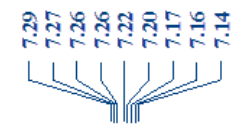

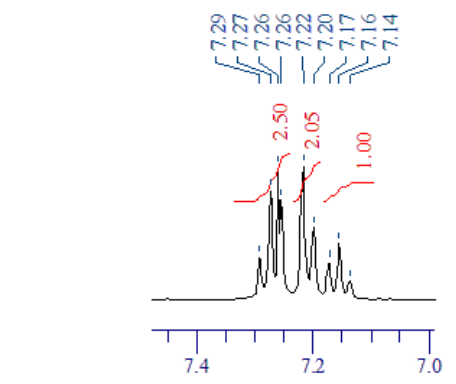
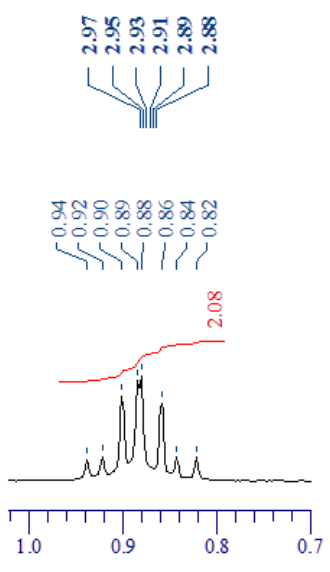
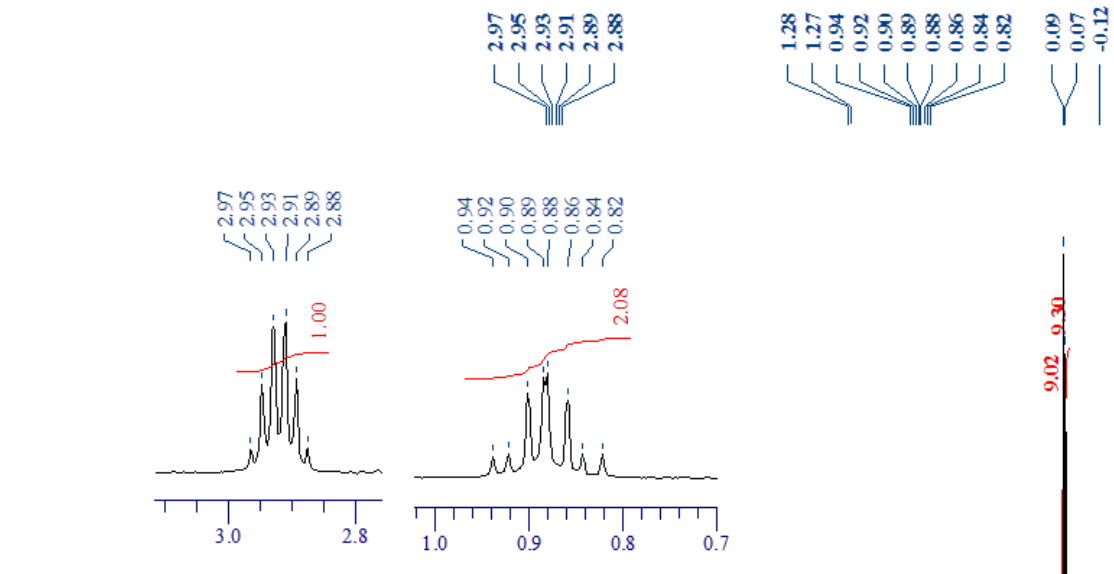

(1)
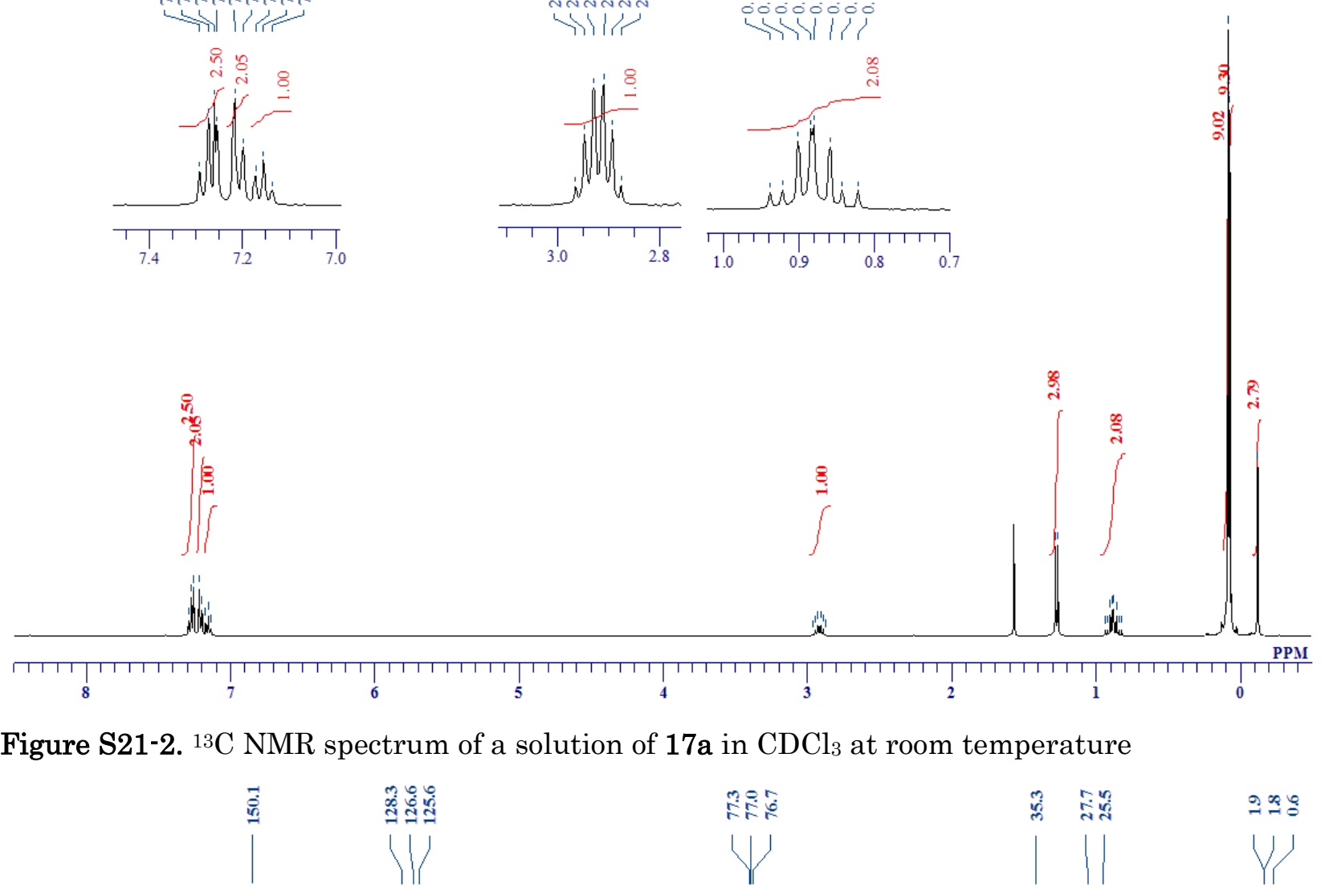

Figure $\mathrm{S} 21-2 .{ }^{13} \mathrm{C} \mathrm{NMR}$ spectrum of a solution of $17 \mathrm{a}$ in $\mathrm{CDCl}_{3}$ at room temperature

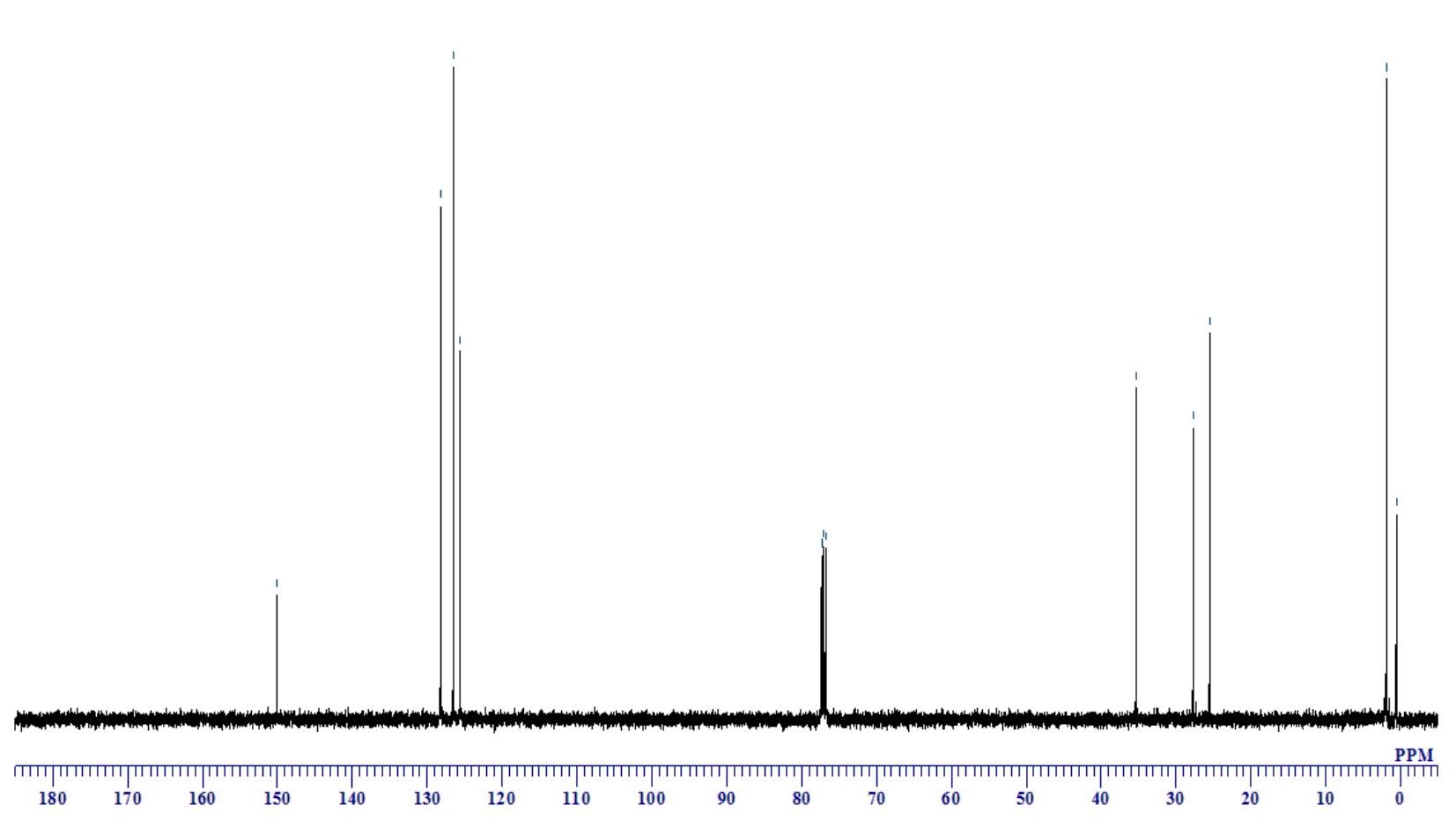


Figure S21-3. ${ }^{29} \mathrm{Si} \mathrm{NMR}$ spectrum of a solution of $17 \mathrm{a}$ in $\mathrm{CDCl}_{3}$ at room temperature

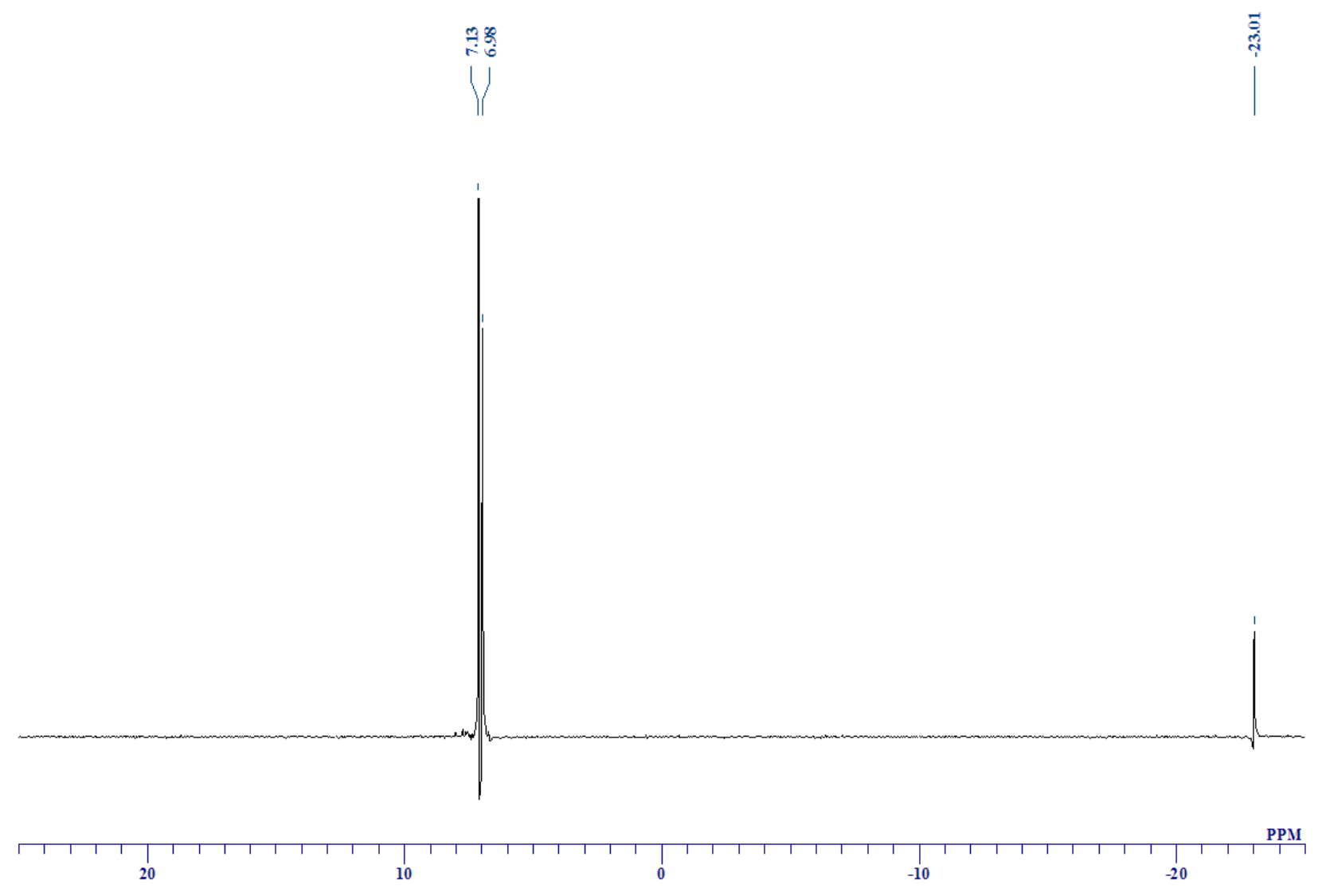

Figure S22-1. ${ }^{1} \mathrm{H}$ NMR spectrum of a solution of $18 \mathrm{a}$ in $\mathrm{CDCl}_{3}$ at room temperature

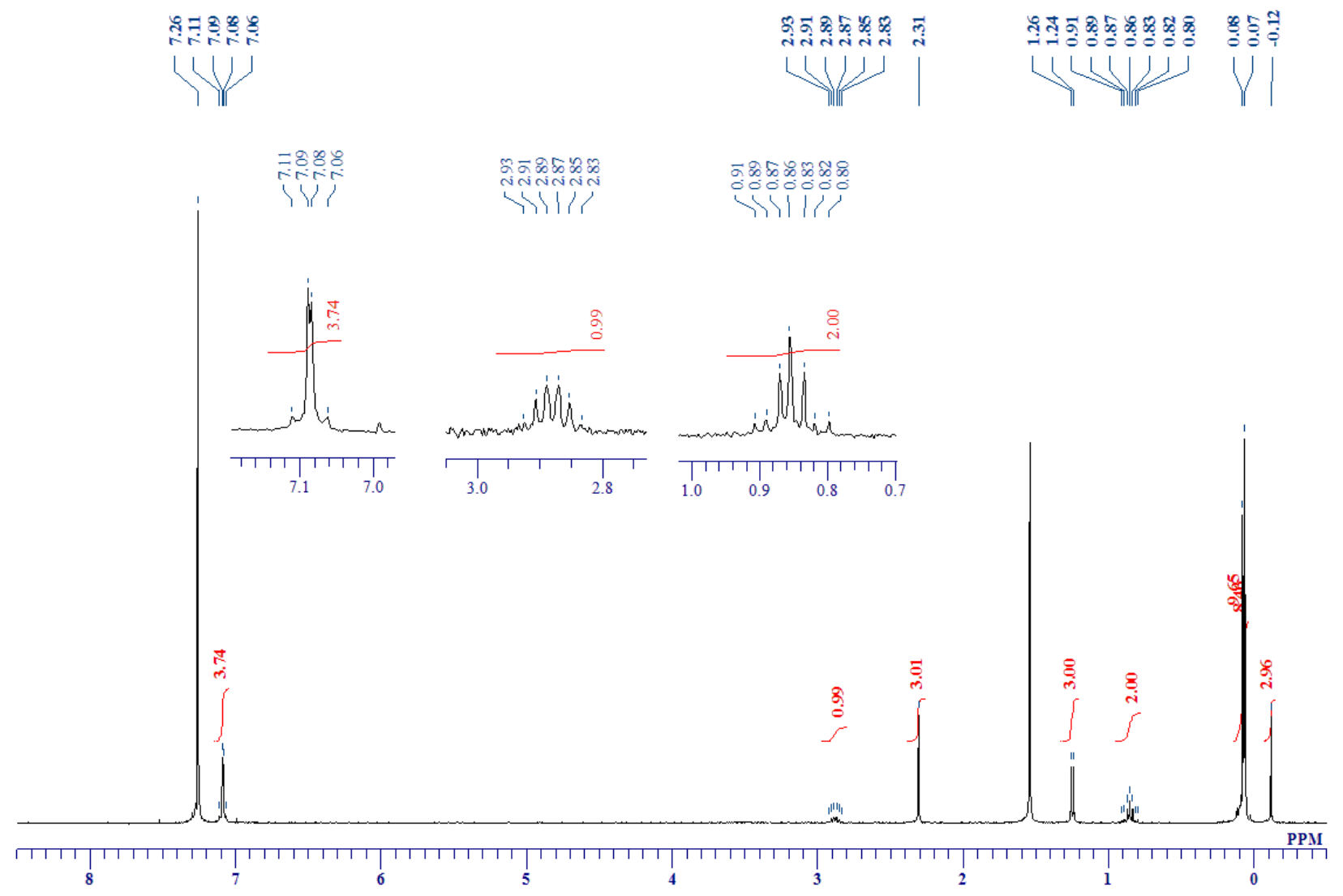


Figure S22-2. ${ }^{13} \mathrm{C}$ NMR spectrum of a solution of $18 \mathrm{a}$ in $\mathrm{CDCl}_{3}$ at room temperature

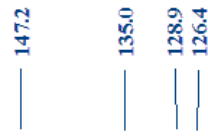

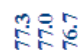

Y)

के

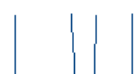

920

(I)
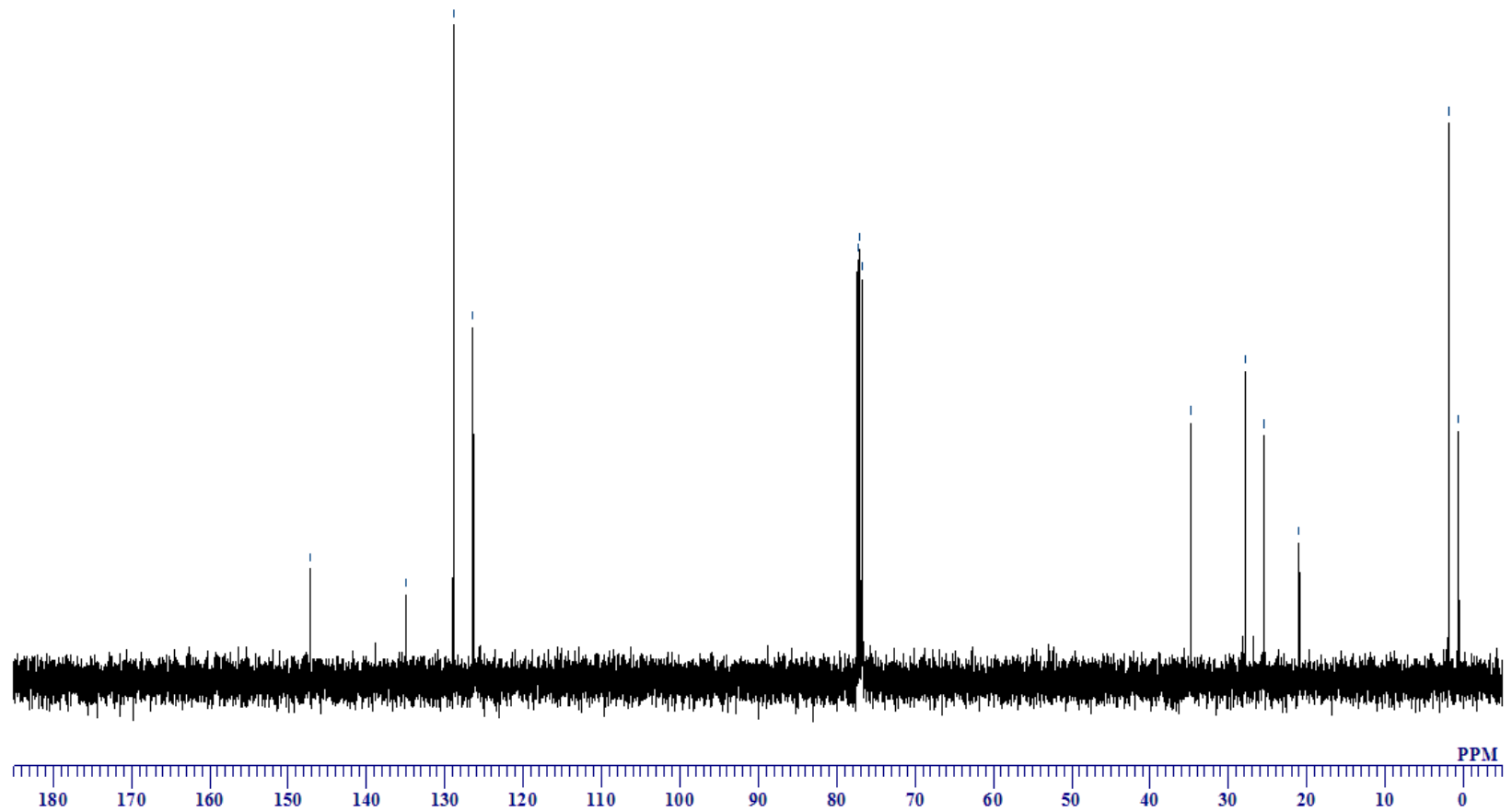

Figure S22-3. ${ }^{29} \mathrm{Si} \mathrm{NMR}$ spectrum of a solution of $18 \mathrm{a}$ in $\mathrm{CDCl}_{3}$ at room temperature

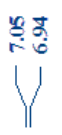

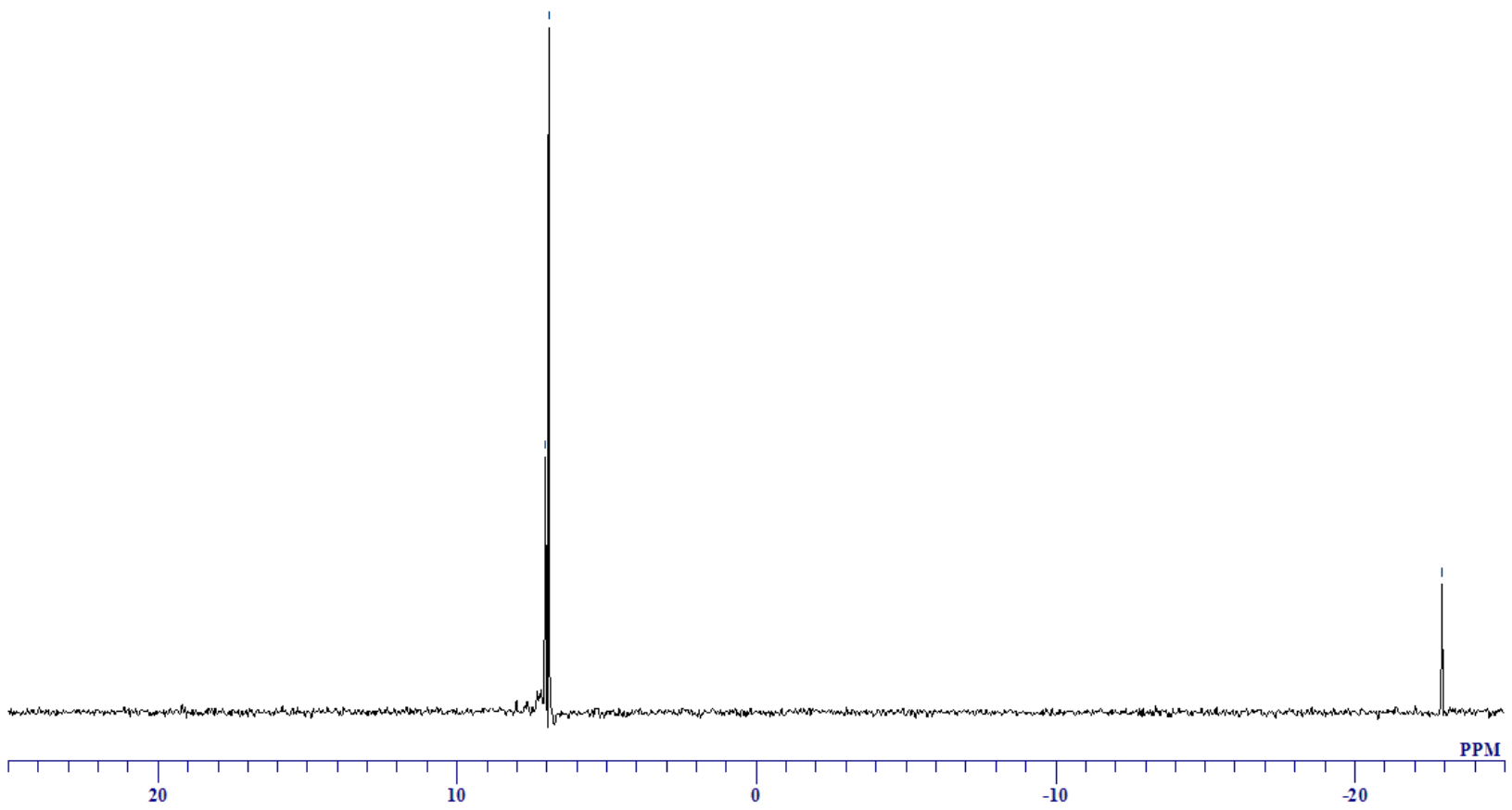


Figure S23-1. ${ }^{1} \mathrm{H}$ NMR spectrum of a solution of $19 \mathrm{a}$ in $\mathrm{CDCl}_{3}$ at room temperature

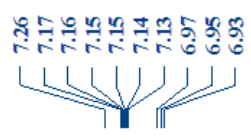

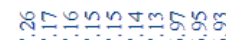
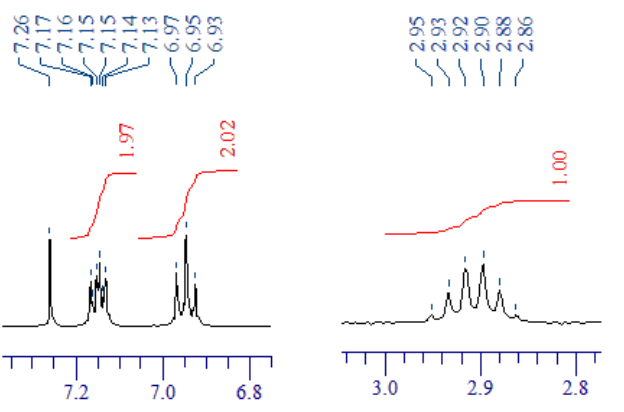

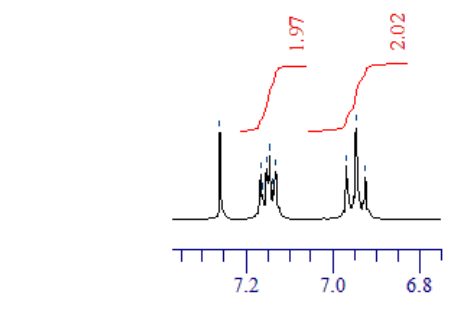

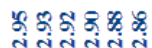

Uiv
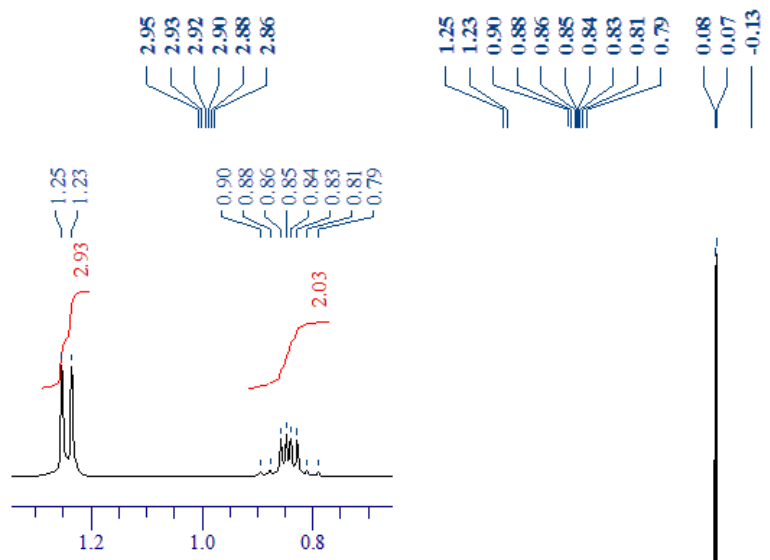

\section{.}

PM

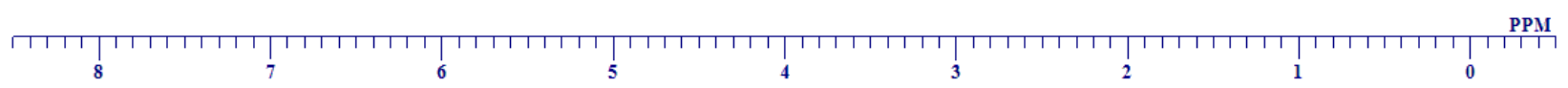

Figure S23-2. ${ }^{13} \mathrm{C}$ NMR spectrum of a solution of $19 \mathrm{a}$ in $\mathrm{CDCl}_{3}$ at room temperature
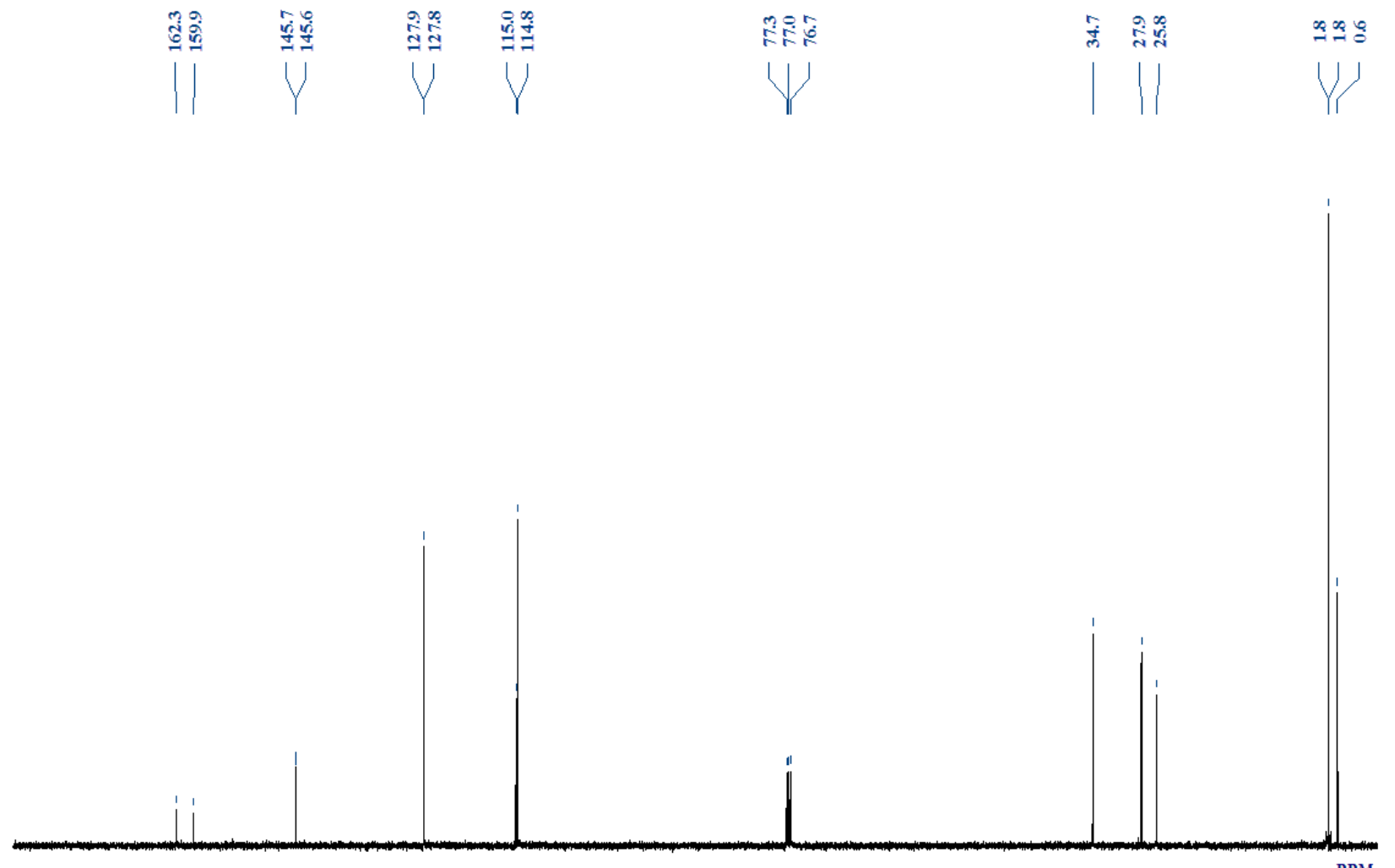

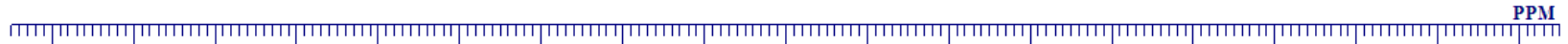

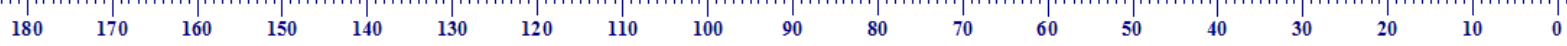


Figure S23-3. ${ }^{19} \mathrm{~F} \mathrm{NMR} \mathrm{spectrum} \mathrm{of} \mathrm{a} \mathrm{solution} \mathrm{of} 19 \mathrm{a}$ in $\mathrm{CDCl}_{3}$ at room temperature

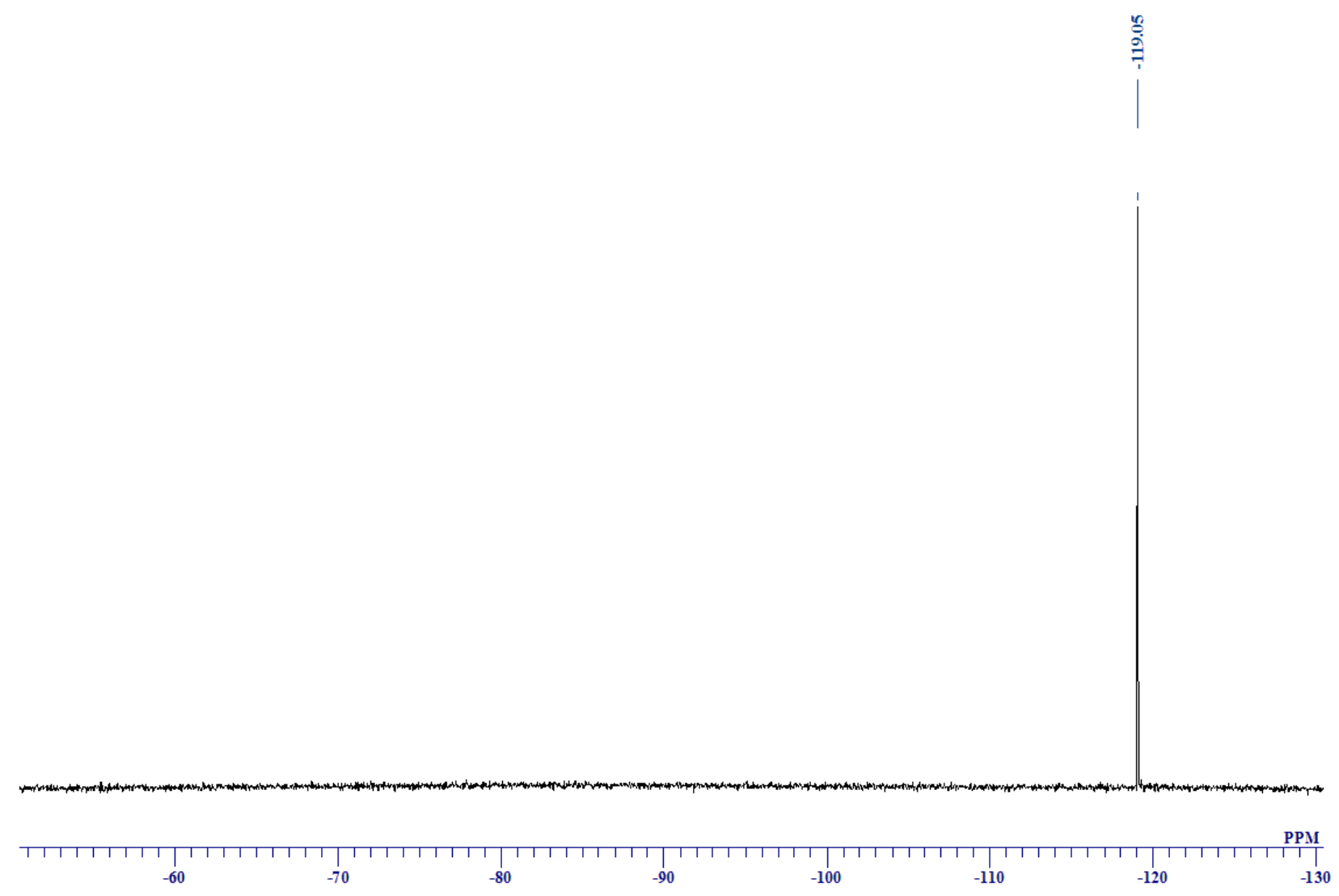

Figure S23-4. ${ }^{29} \mathrm{Si} \mathrm{NMR}$ spectrum of a solution of $19 \mathrm{a}$ in $\mathrm{CDCl}_{3}$ at room temperature
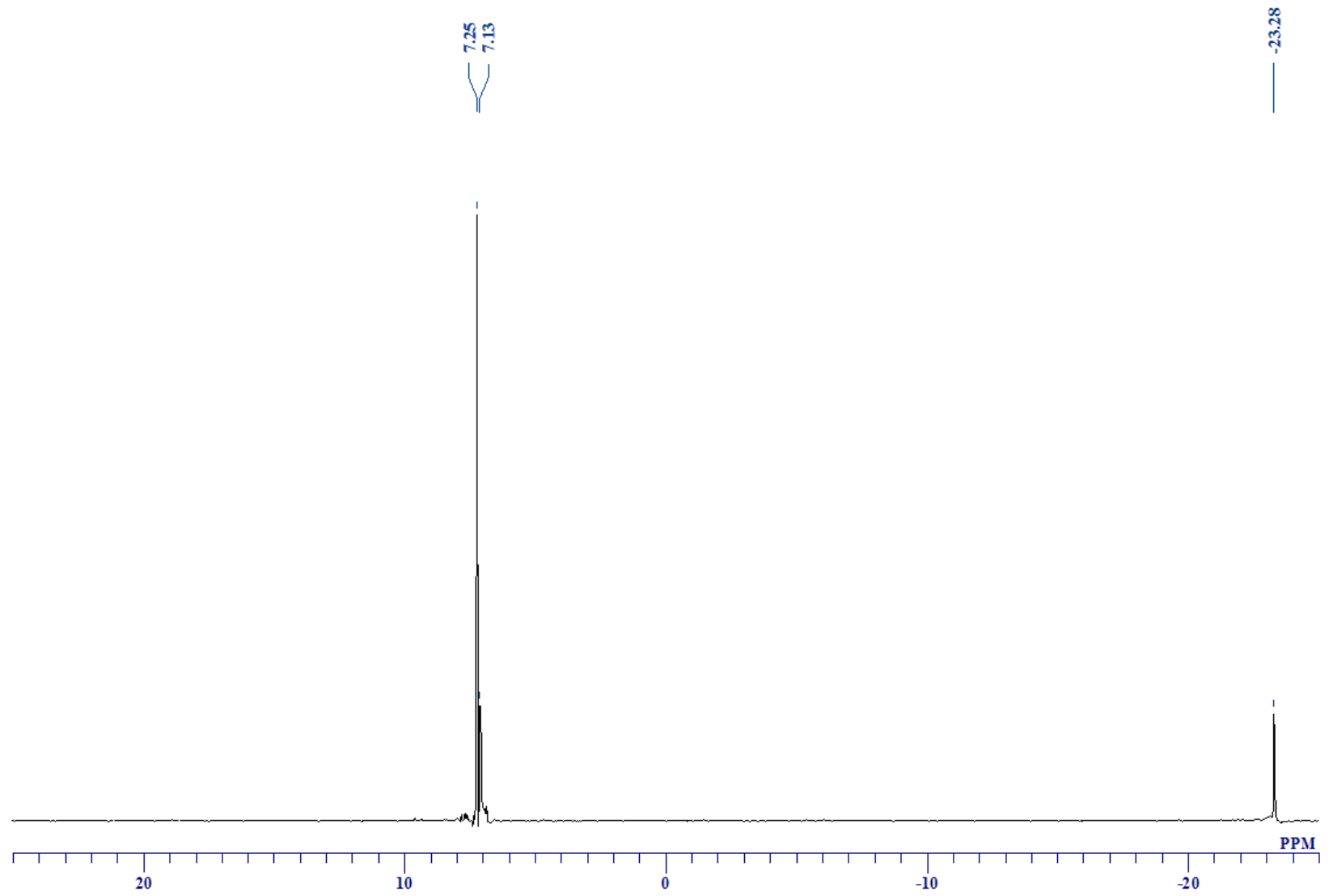
Figure S24-1. ${ }^{1} \mathrm{H}$ NMR spectrum of a solution of $20 \mathrm{a}$ in $\mathrm{CDCl}_{3}$ at room temperature

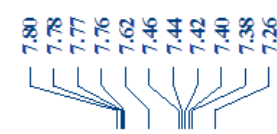

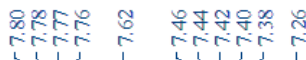

पif i viliti
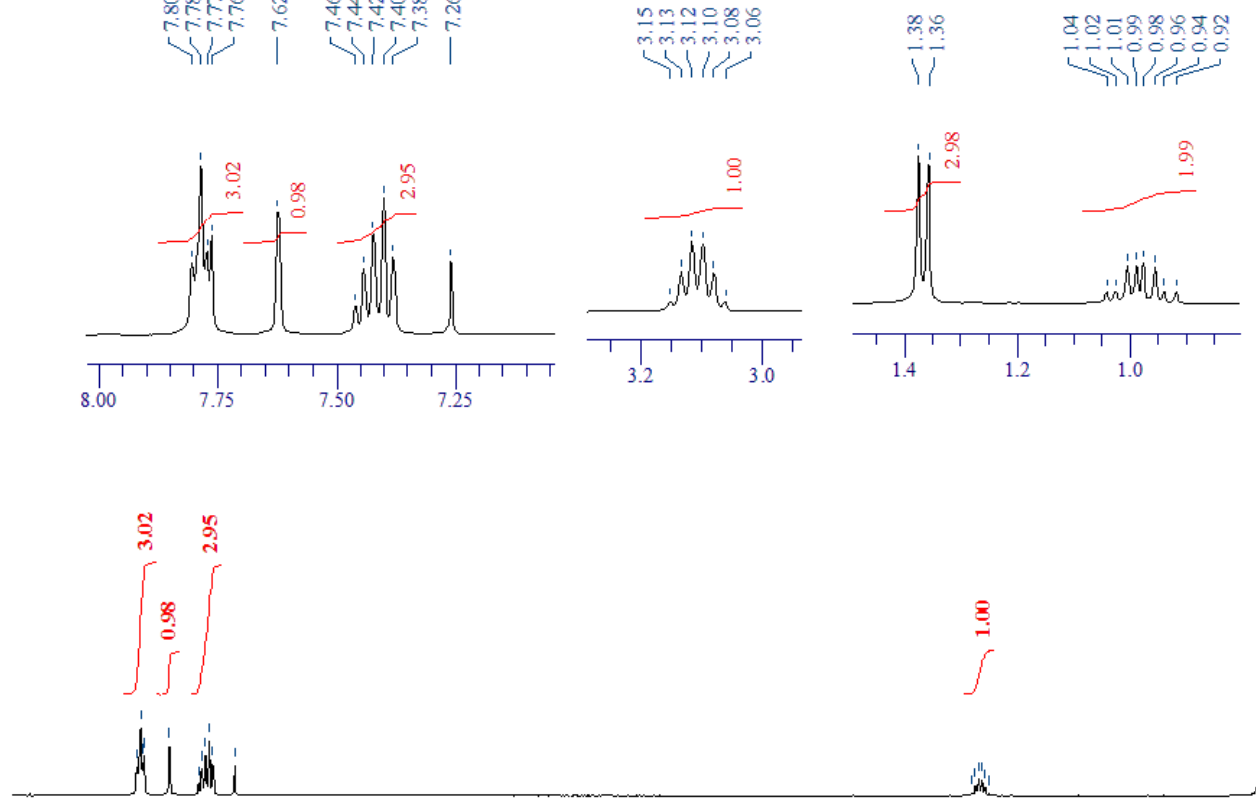

Figure S24-2. ${ }^{13} \mathrm{C}$ NMR spectrum of a solution of $20 \mathrm{a}$ in $\mathrm{CDCl}_{3}$ at room temperature
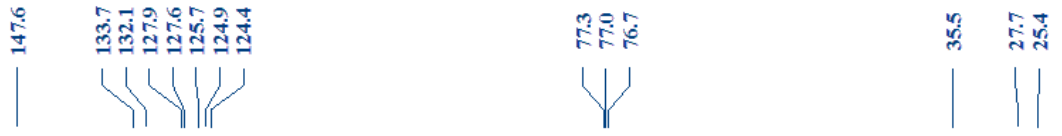

29

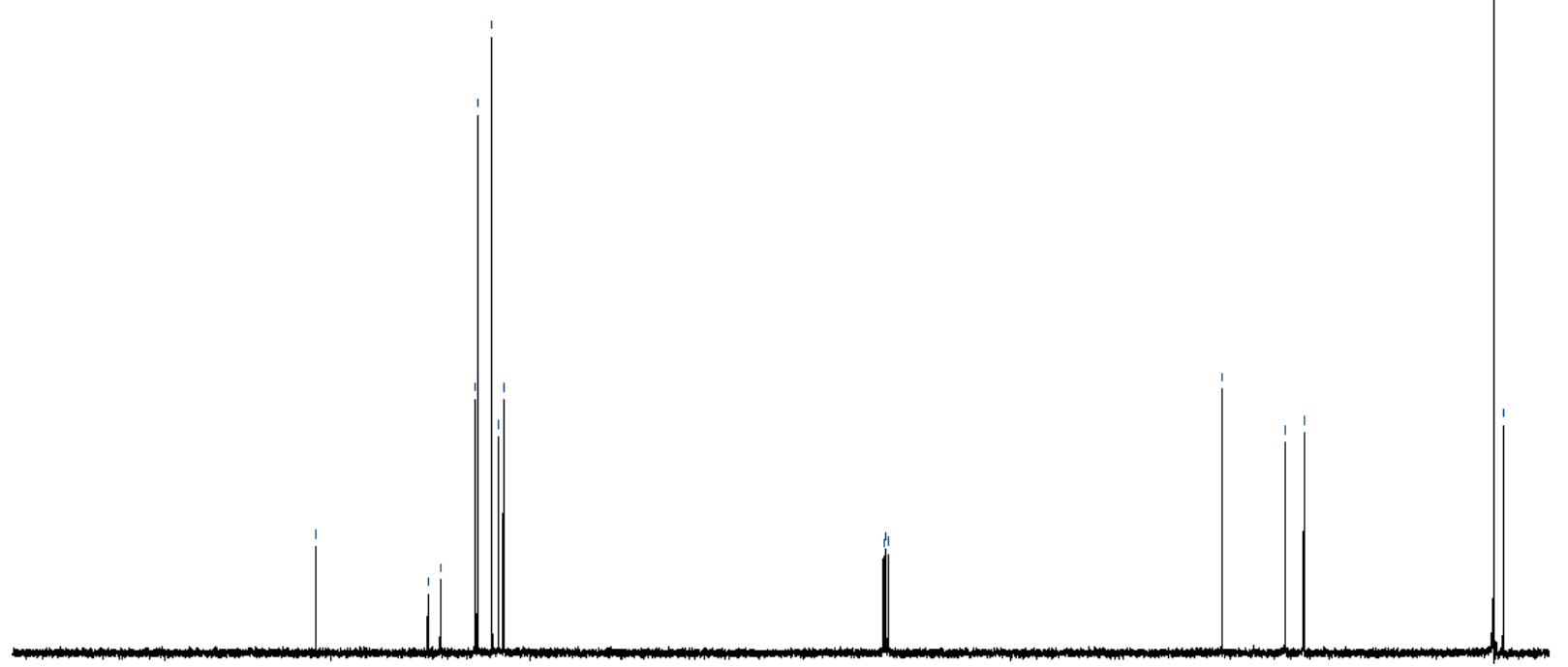

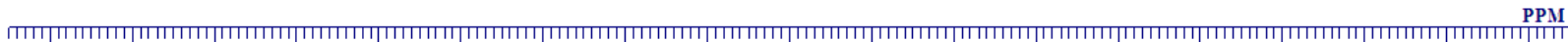
$180 \quad 170$

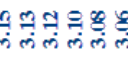

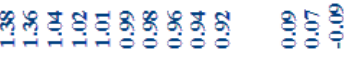

un

PPM

IIIT 
Figure S24-3. ${ }^{29} \mathrm{Si} \mathrm{NMR}$ spectrum of a solution of $20 \mathrm{a}$ in $\mathrm{CDCl}_{3}$ at room temperature

$$
\text { in }
$$

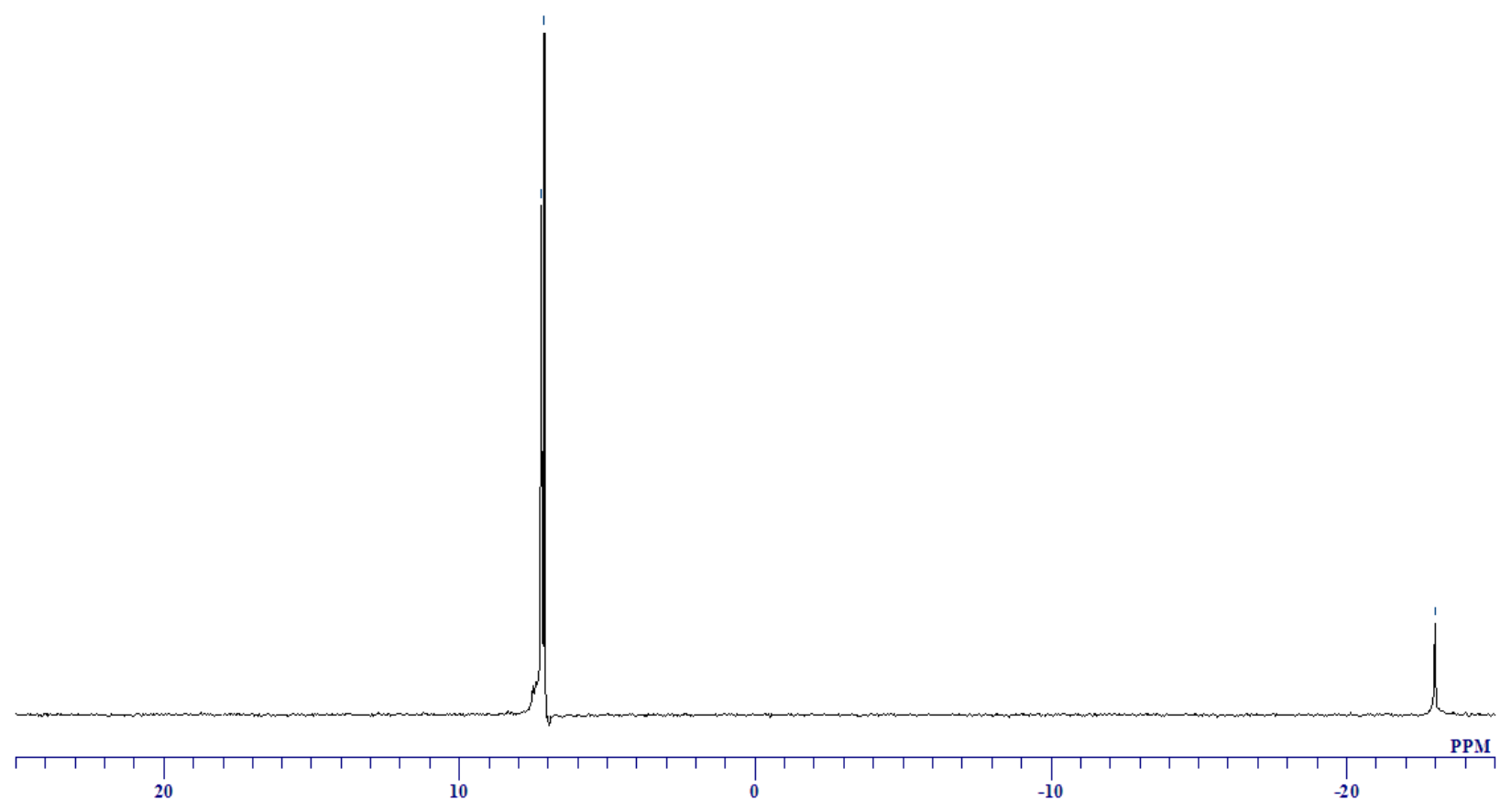

Figure S25-1. ${ }^{1} \mathrm{H}$ NMR spectrum of a solution of $21 \mathrm{a}$ in $\mathrm{CDCl}_{3}$ at room temperature

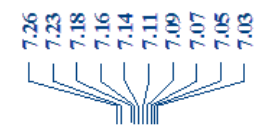

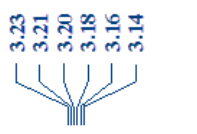
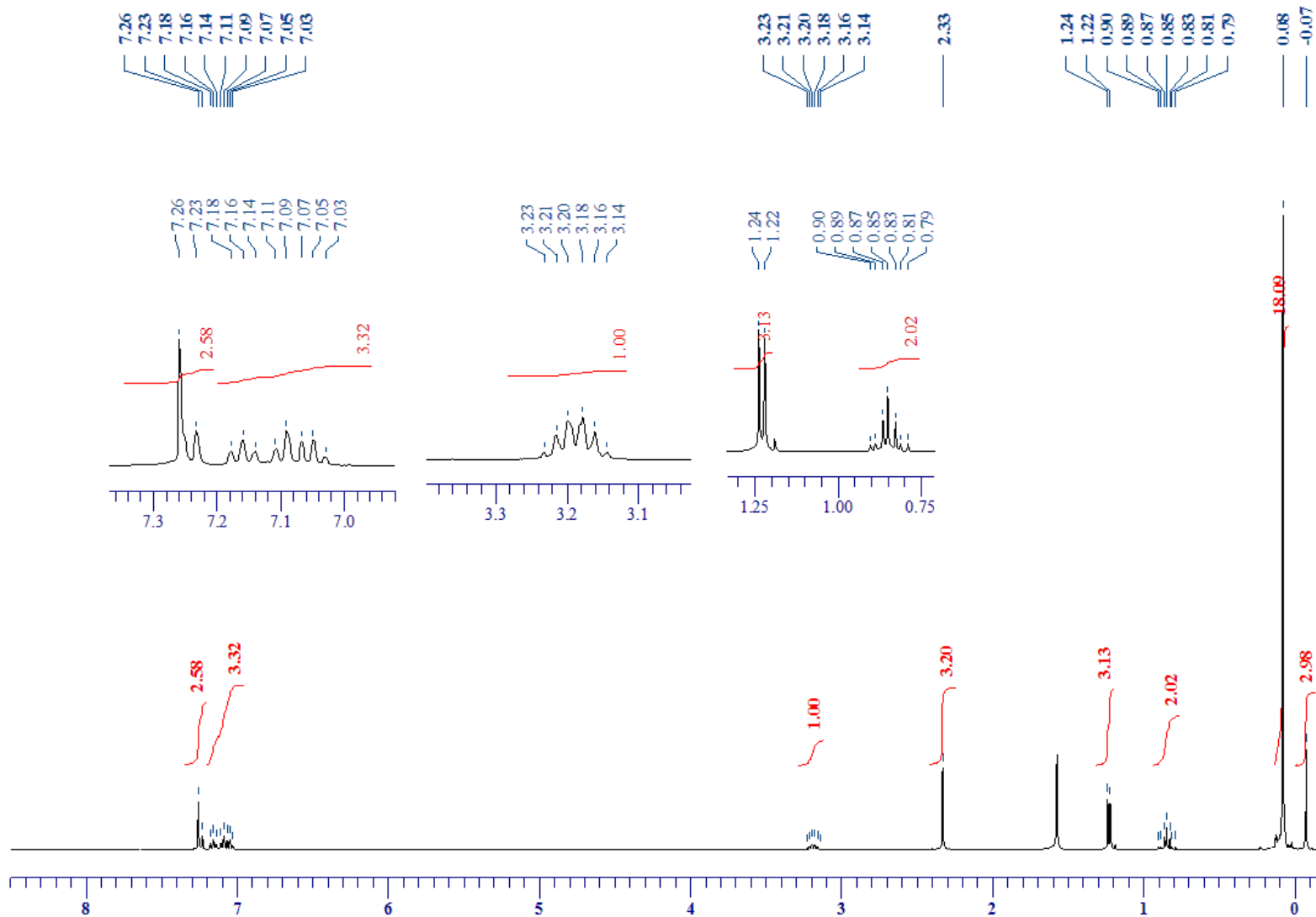
Figure S25-2. ${ }^{13} \mathrm{C}$ NMR spectrum of a solution of 21a in $\mathrm{CDCl}_{3}$ at room temperature
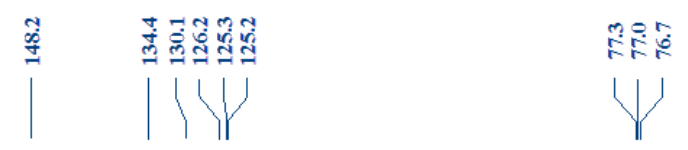

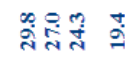

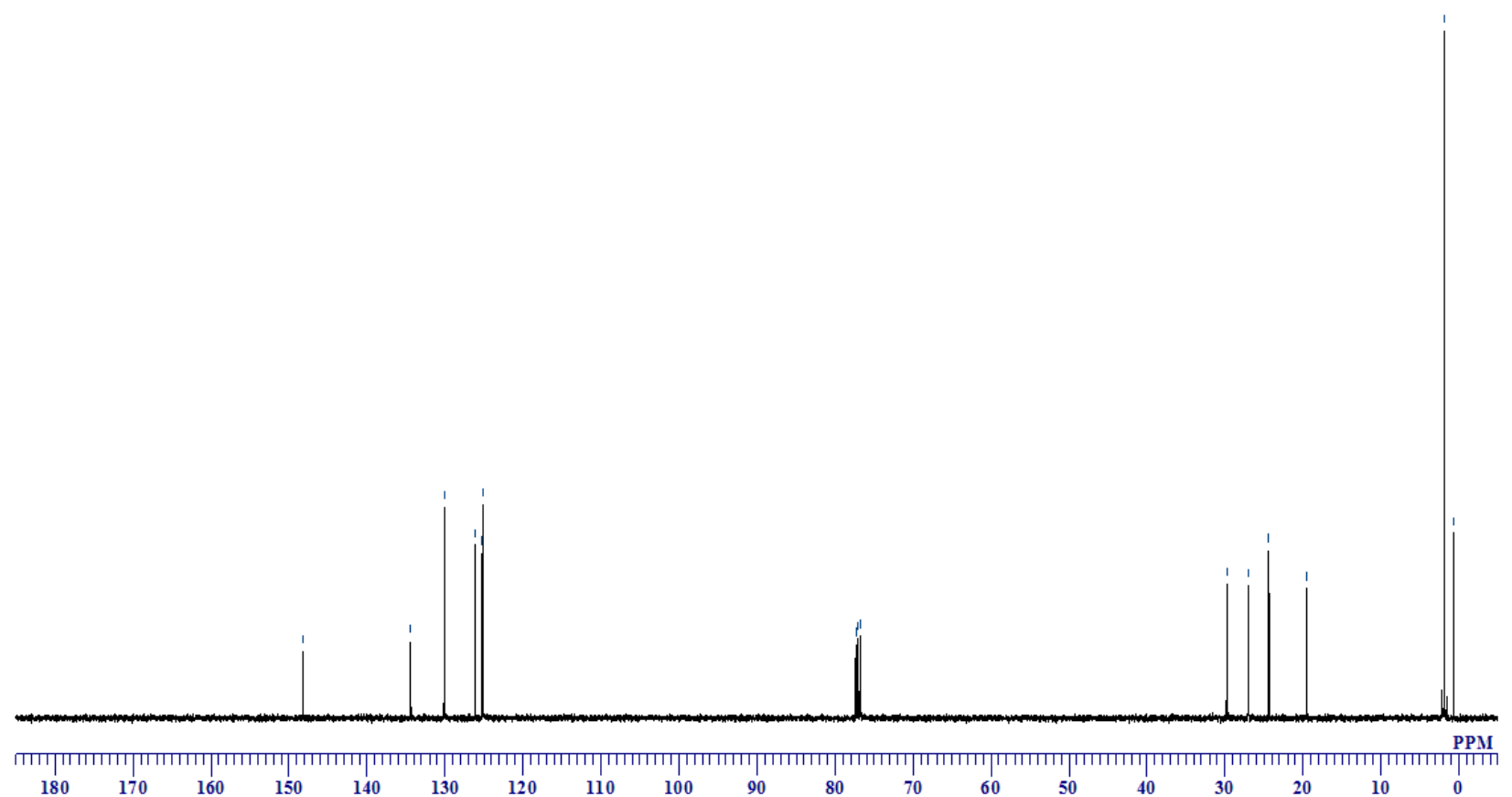

Figure S25-3. ${ }^{29} \mathrm{Si} \mathrm{NMR}$ spectrum of a solution of $21 \mathrm{a}$ in $\mathrm{CDCl}_{3}$ at room temperature
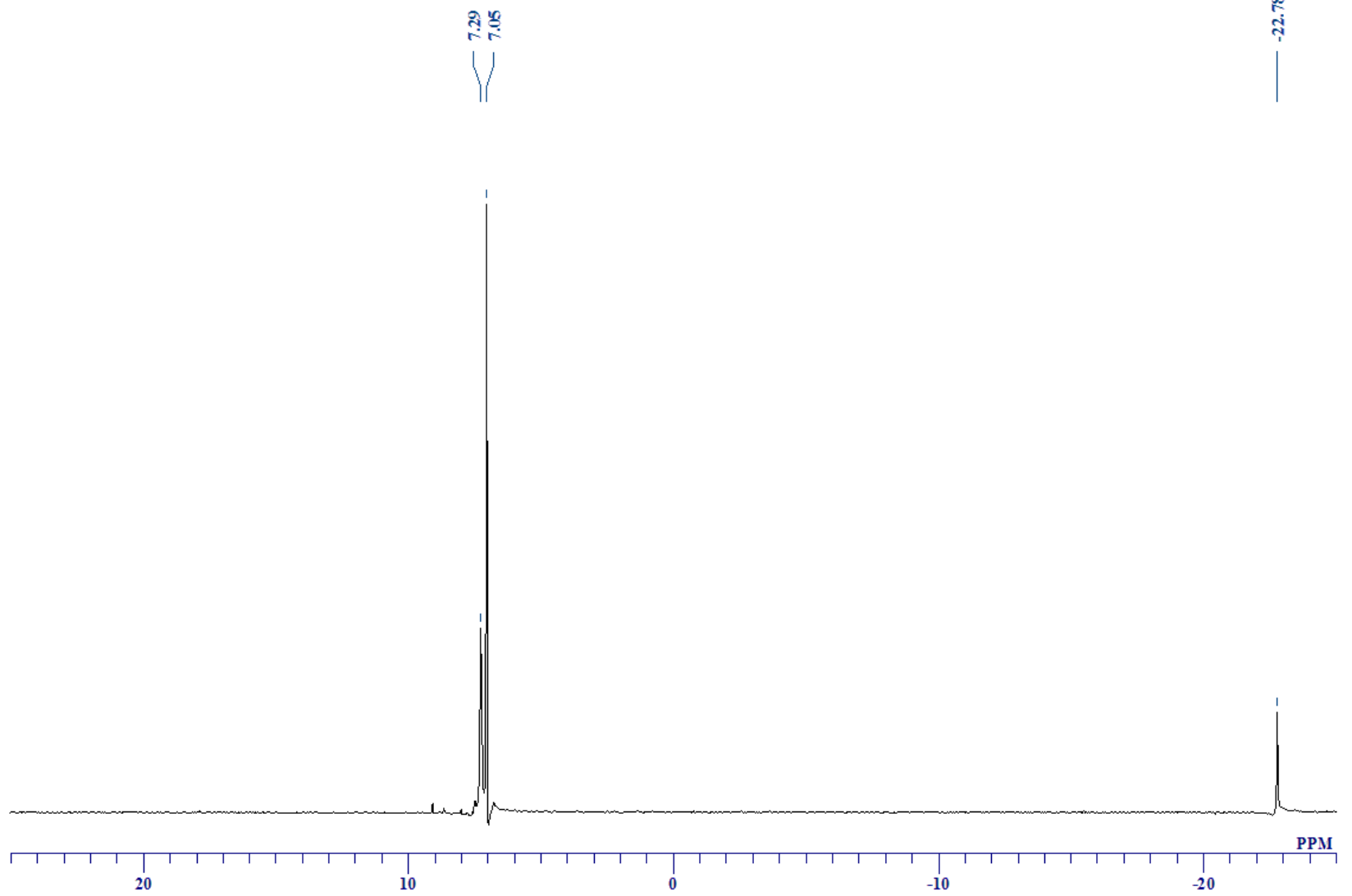
Figure $\mathrm{S} 26-1 .{ }^{1} \mathrm{H}$ NMR spectrum of a solution of $22 \mathrm{a}$ in $\mathrm{CDCl}_{3}$ at room temperature

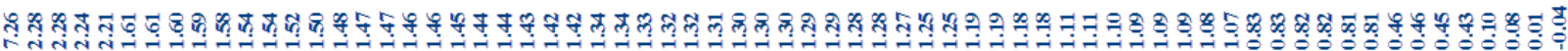

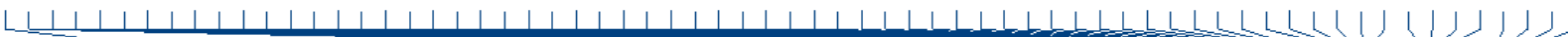
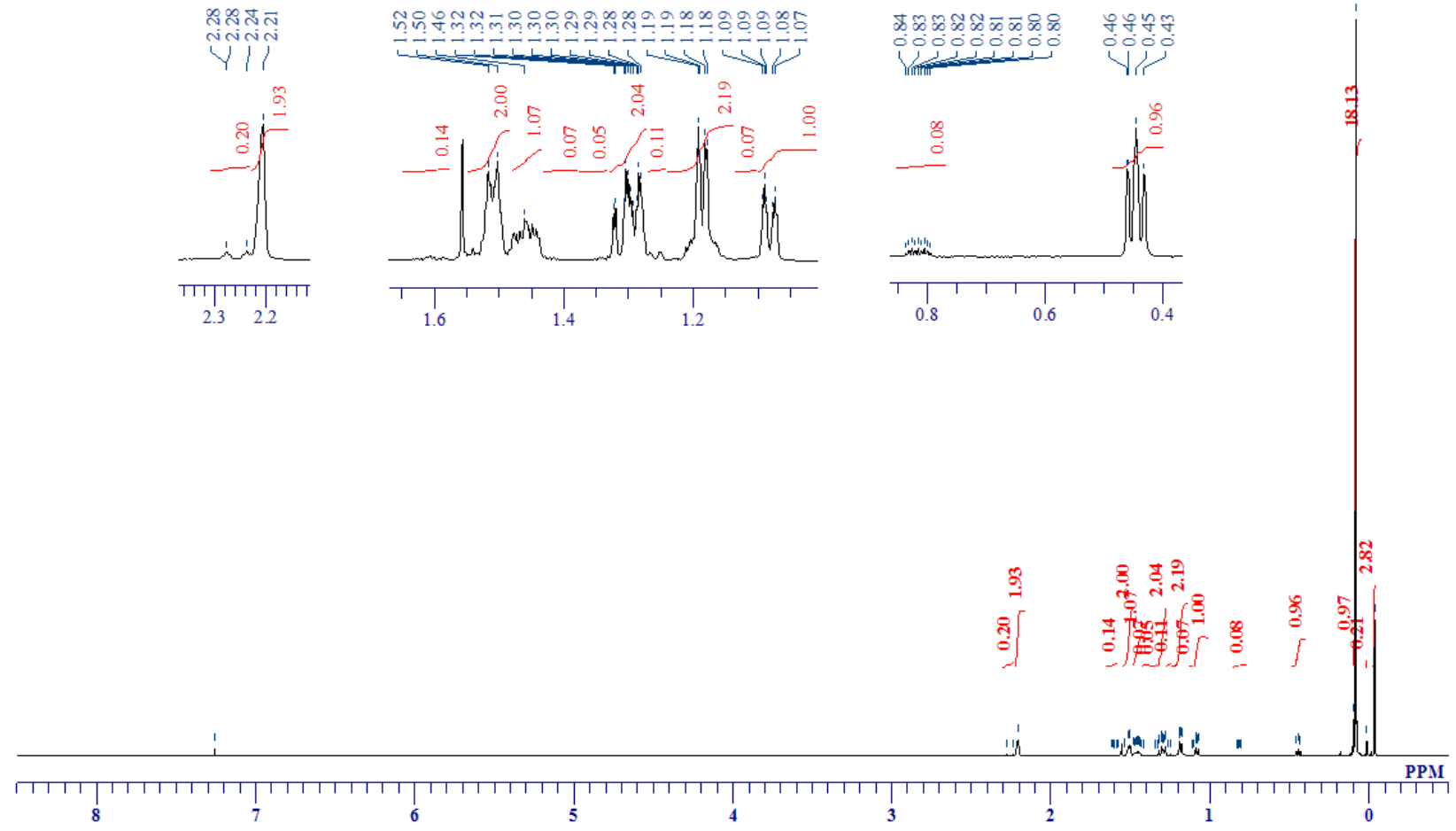

Figure S26-2. ${ }^{13} \mathrm{C}$ NMR spectrum of a solution of $22 \mathrm{a}$ in $\mathrm{CDCl}_{3}$ at room temperature
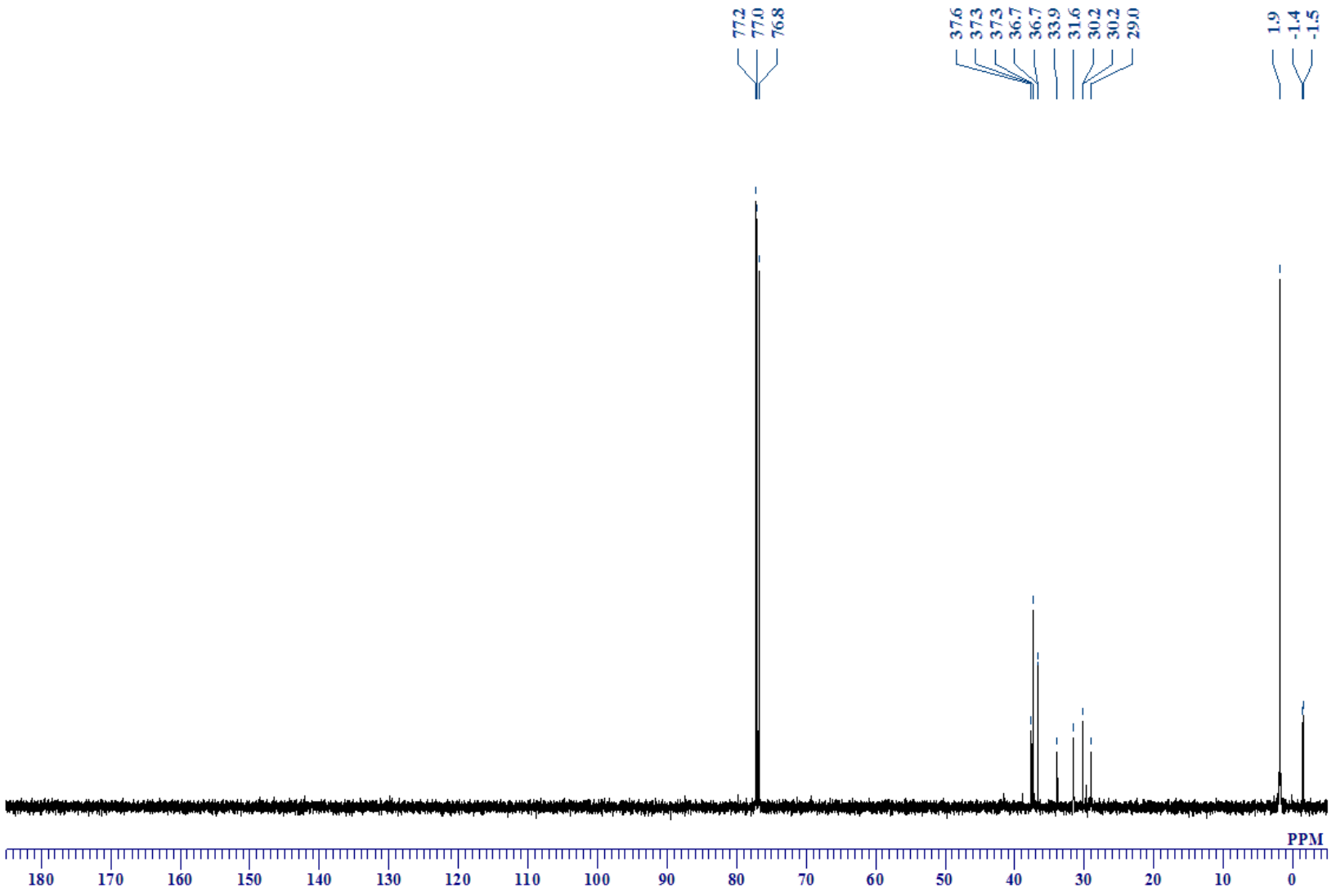
Figure S26-3. ${ }^{29} \mathrm{Si} \mathrm{NMR}$ spectrum of a solution of $22 \mathrm{a}$ in $\mathrm{CDCl}_{3}$ at room temperature

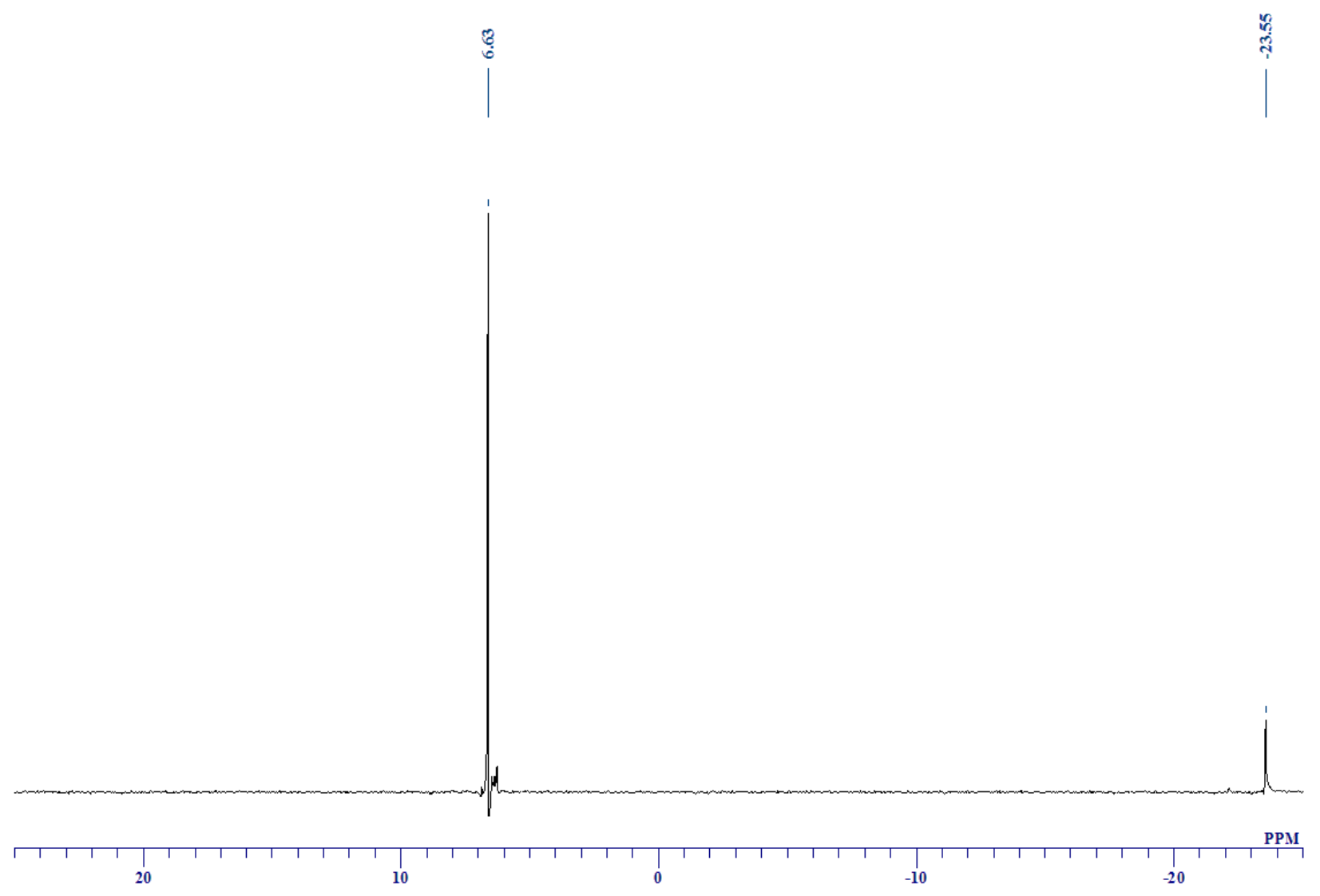

Figure $\mathrm{S} 26-4 .{ }^{1} \mathrm{H}^{-1} \mathrm{H}$ cosy $\mathrm{NMR}$ spectrum of a solution of $22 \mathrm{a}$ in $\mathrm{CDCl}_{3}$ at room temperature

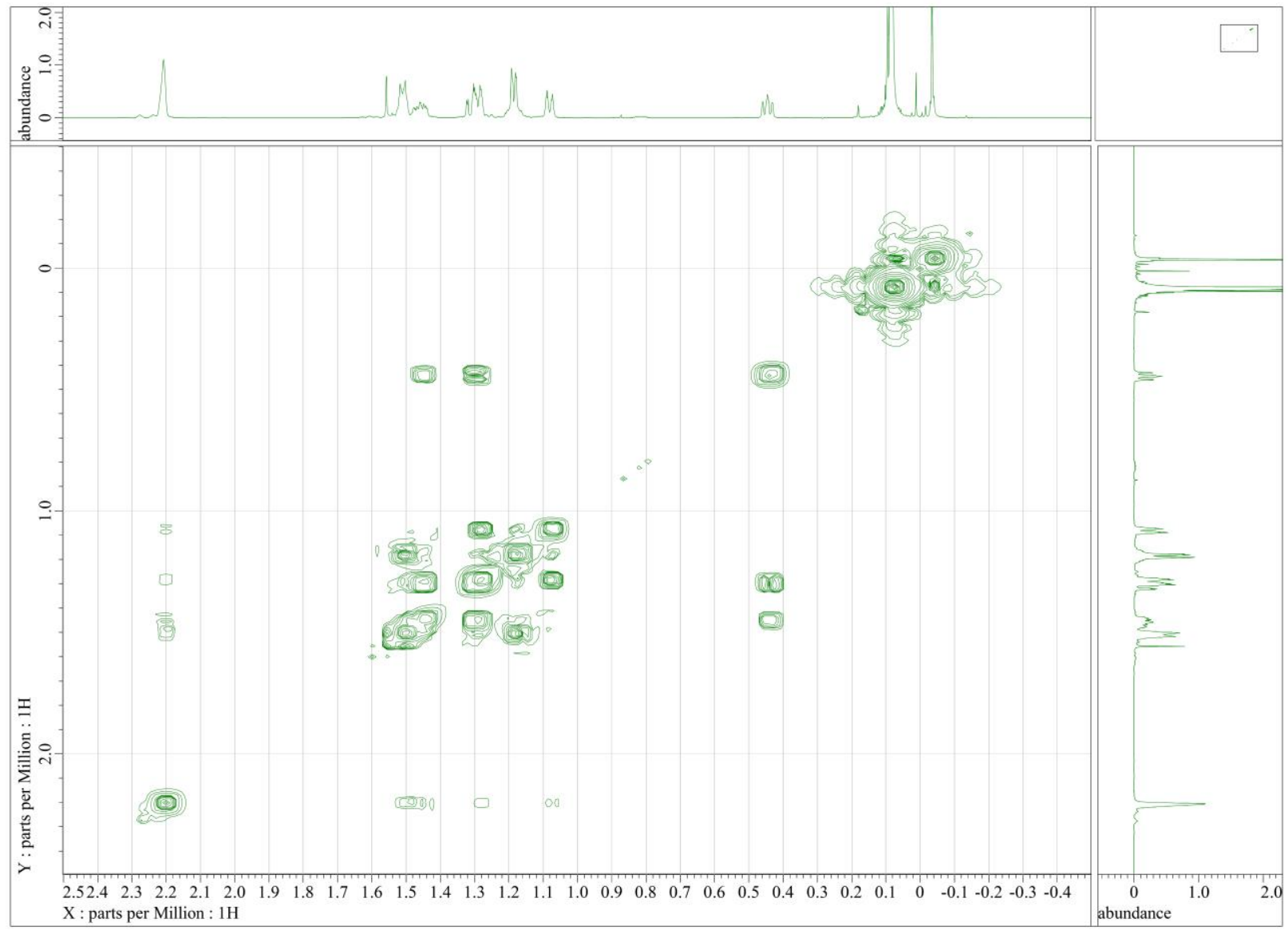


Figure $\mathrm{S} 27-1 .{ }^{1} \mathrm{H}$ NMR spectrum of a solution of $23 \mathrm{a}$ in $\mathrm{CDCl}_{3}$ at room temperature

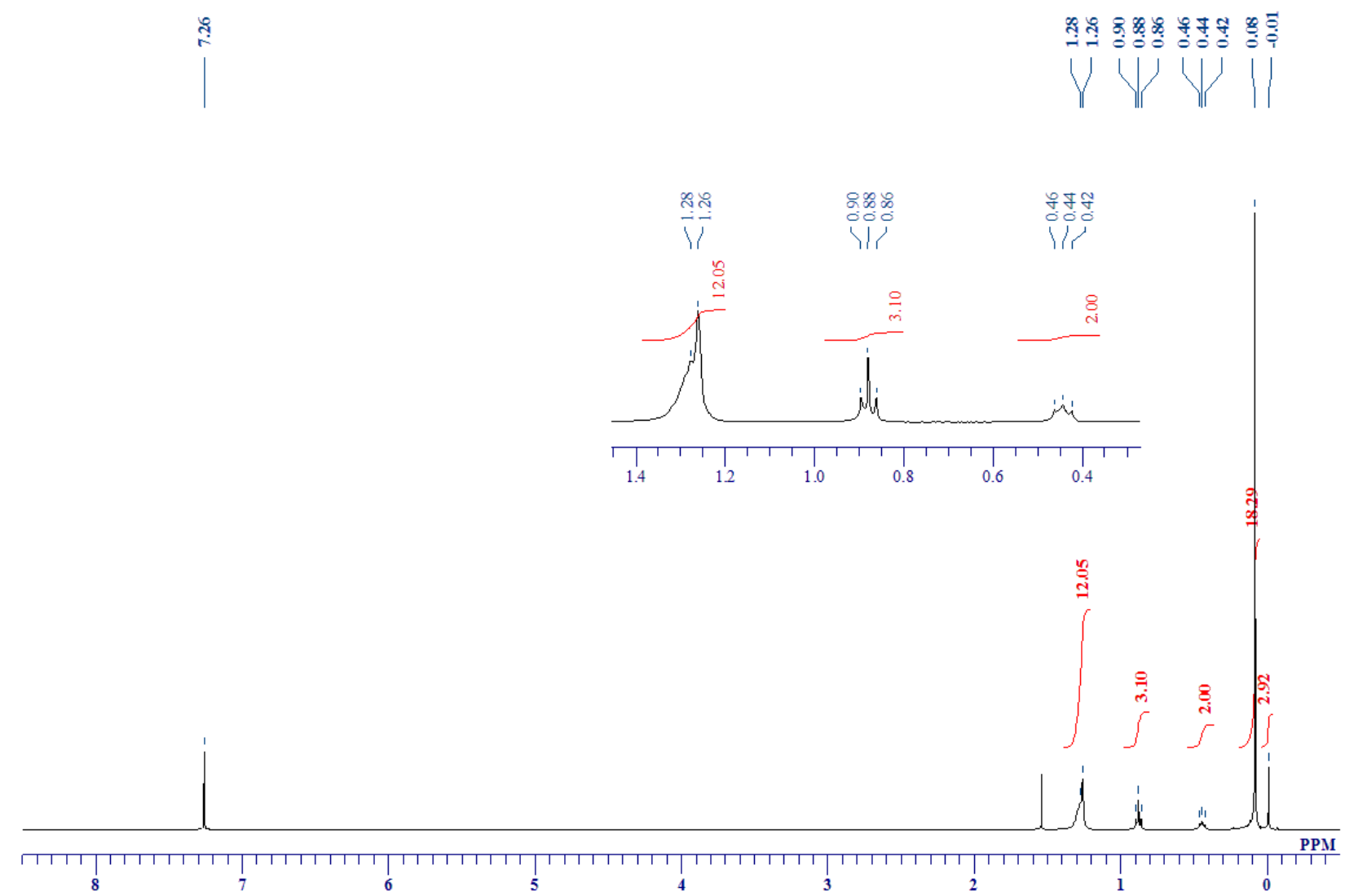

Figure $\mathrm{S} 27-2 .{ }^{13} \mathrm{C}$ NMR spectrum of a solution of $23 \mathrm{a}$ in $\mathrm{CDCl}_{3}$ at room temperature
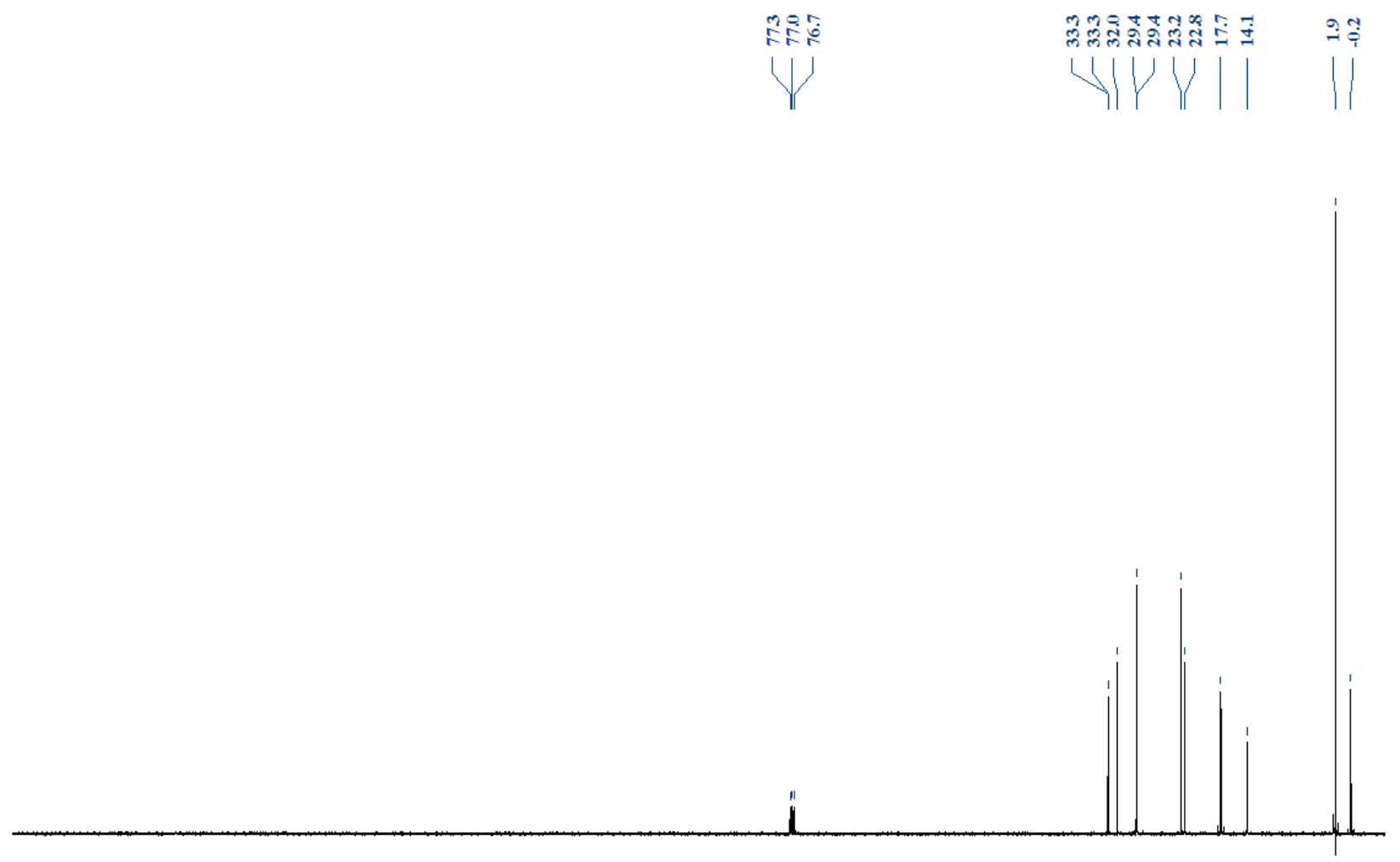

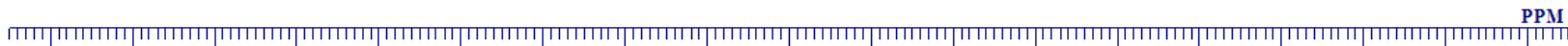

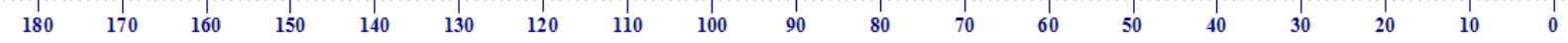


Figure S27-3. ${ }^{29} \mathrm{Si} \mathrm{NMR}$ spectrum of a solution of $23 \mathrm{a}$ in $\mathrm{CDCl}_{3}$ at room temperature

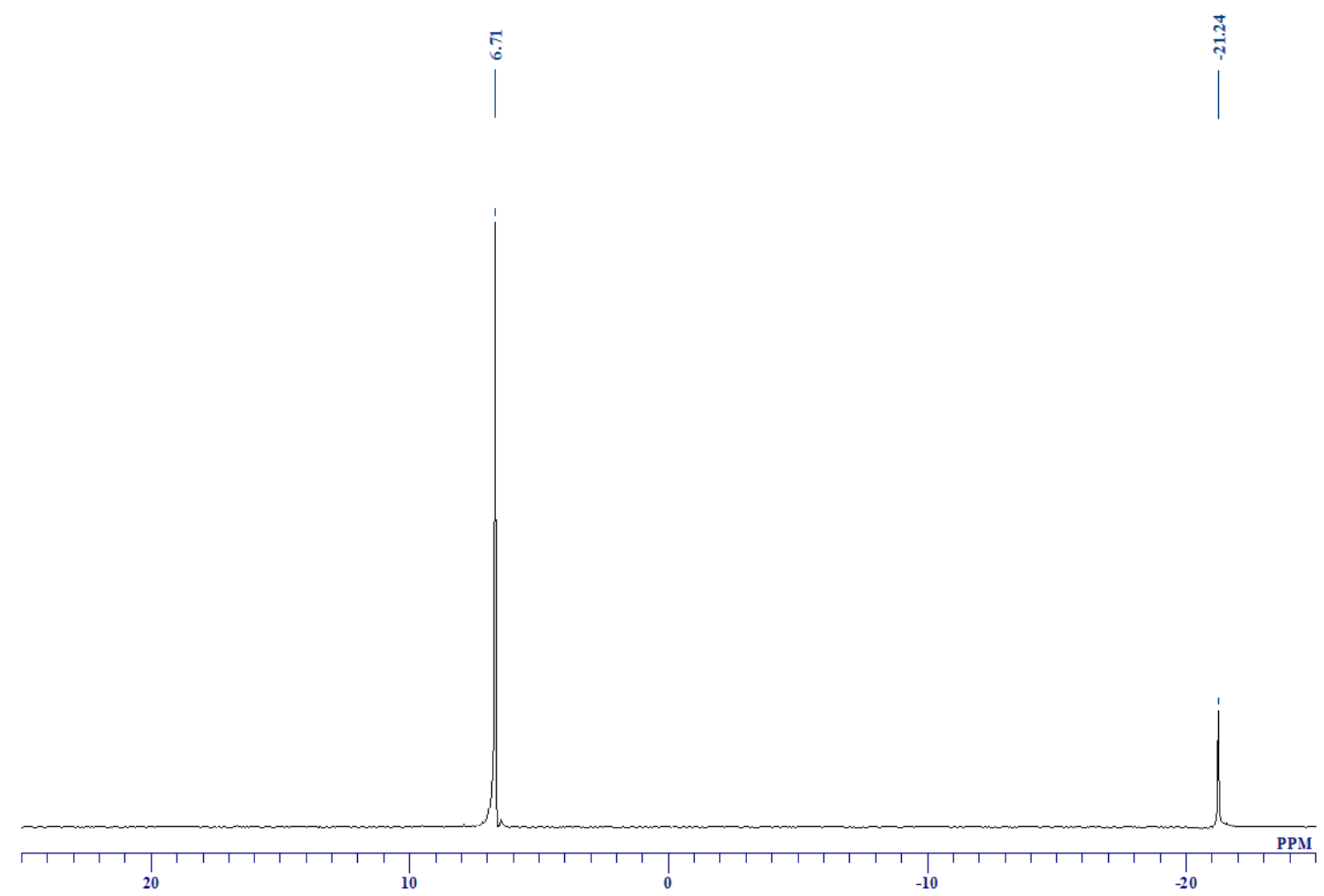

Figure S28-1. ${ }^{1} \mathrm{H}$ NMR spectrum of a solution of $\alpha$-methylstyrene modified silicone $24 \mathrm{a}$ in $\mathrm{CDCl}_{3}$ at room temperature

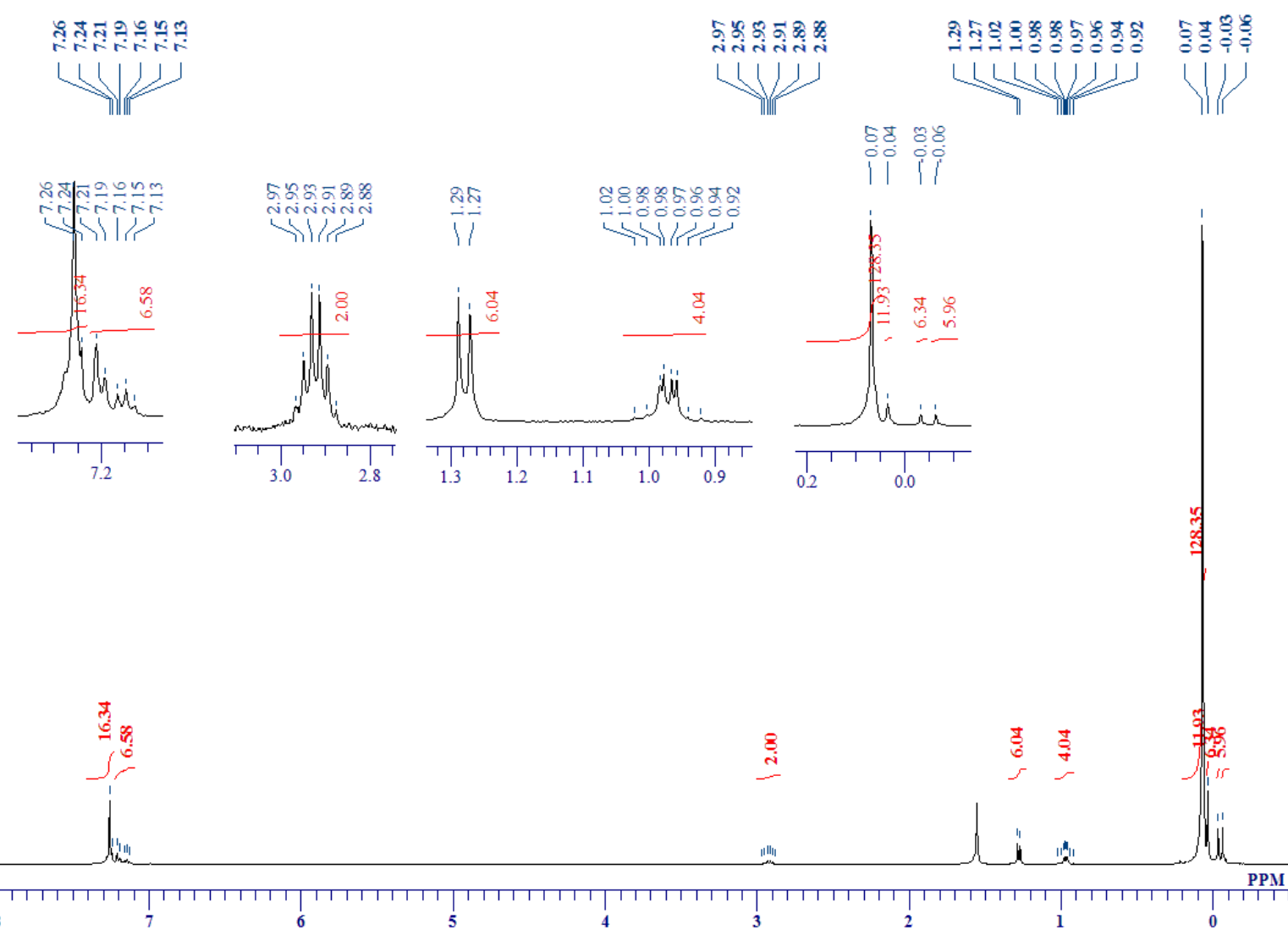


Figure S28-2. ${ }^{13} \mathrm{C}$ NMR spectrum of a solution of $\alpha$-methylstyrene modified silicone $24 \mathrm{a}$ in $\mathrm{CDCl}_{3}$ at room temperature
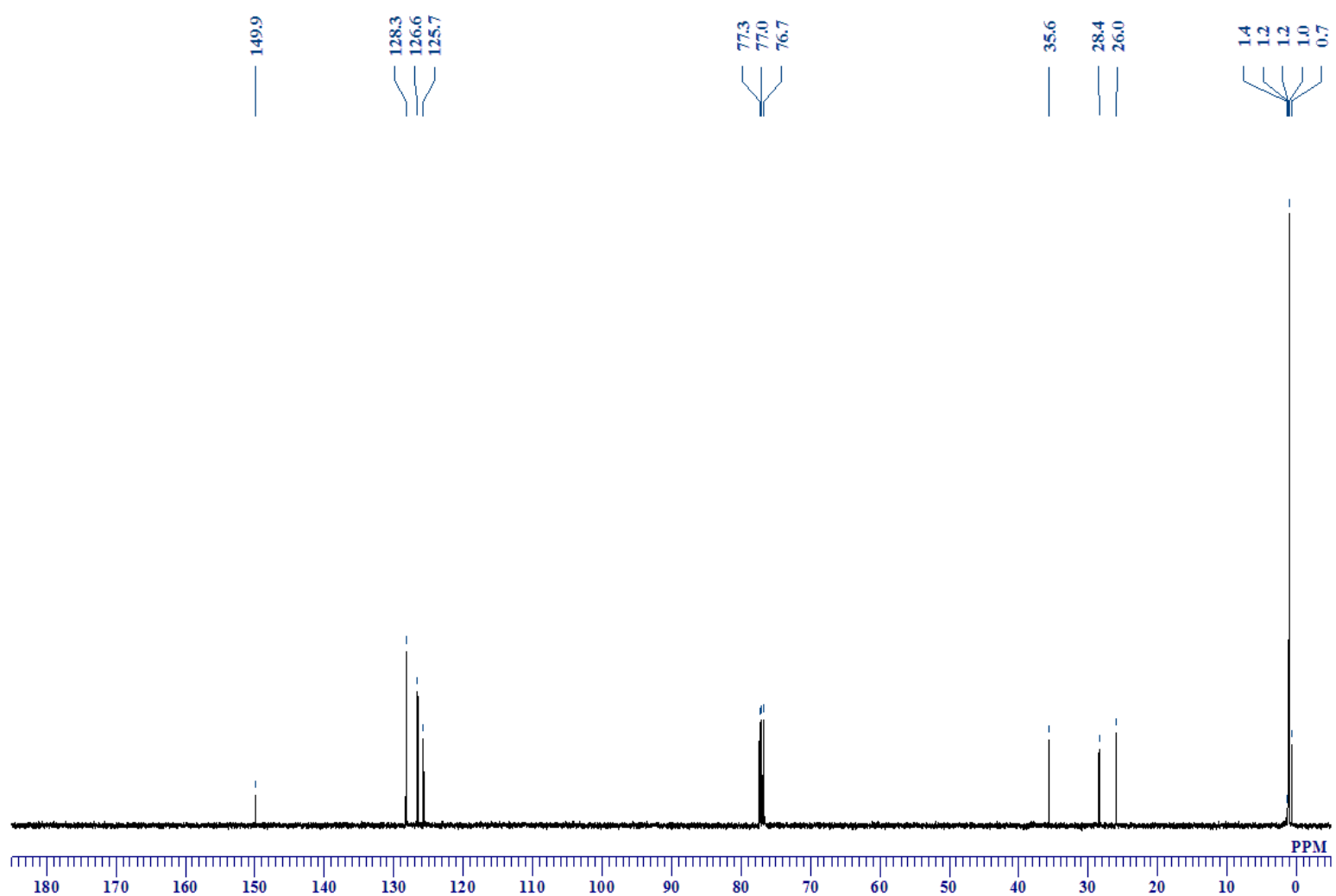

Figure S28-3. ${ }^{29} \mathrm{Si}$ NMR spectrum of a solution of a-methylstyrene modified silicone $24 \mathrm{a}$ in $\mathrm{CDCl}_{3}$ at room temperature
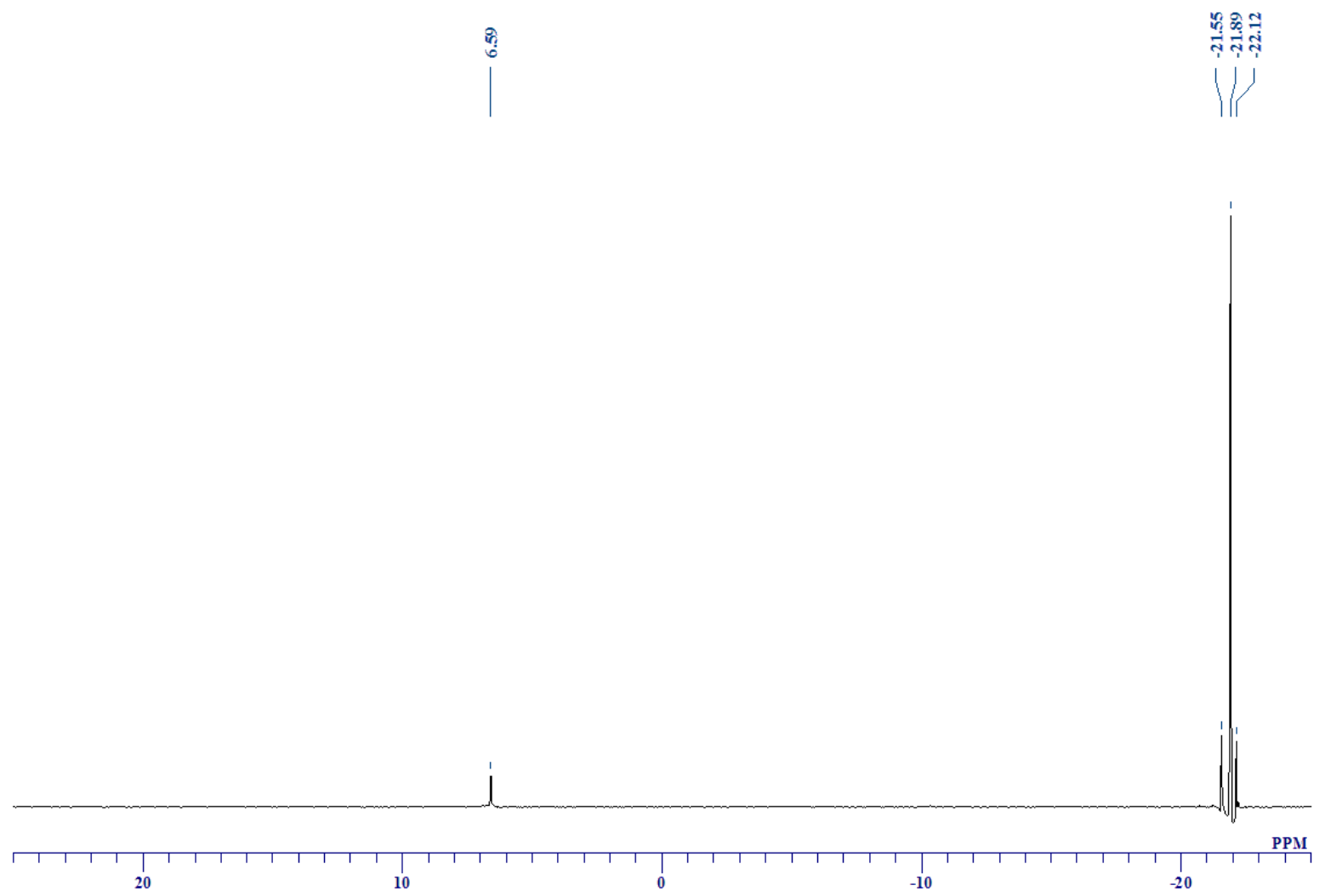
Figure S29-1. ${ }^{1} \mathrm{H}$ NMR spectrum of a solution of styrene modified silicone $25 \mathrm{a}$ in $\mathrm{CDCl}_{3}$ at room temperature
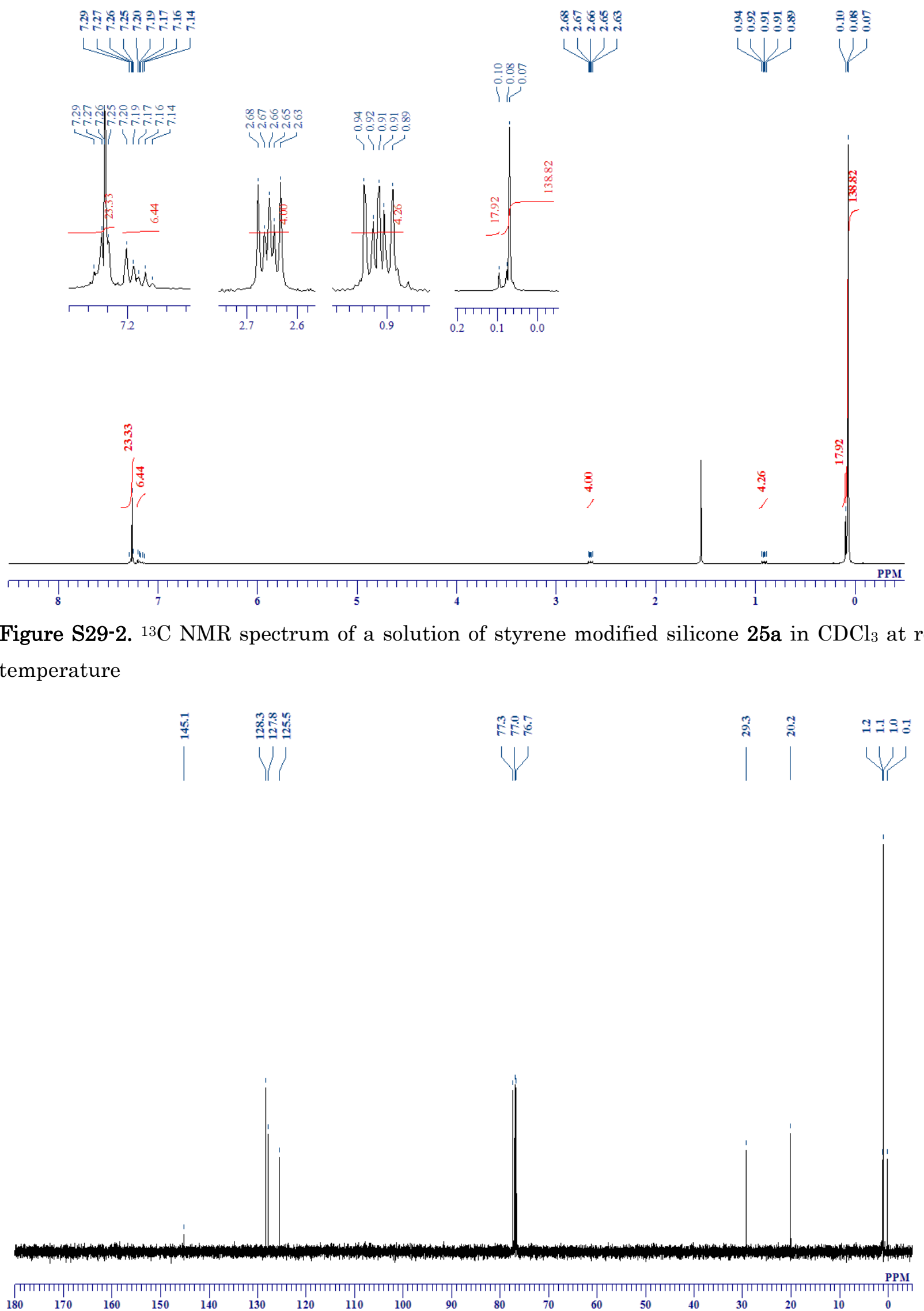

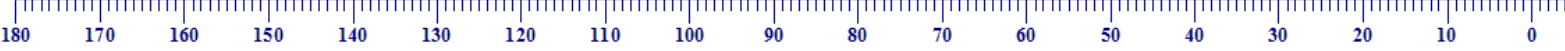


Figure S29-3. ${ }^{29} \mathrm{Si}$ NMR spectrum of a solution of styrene modified silicone $25 \mathrm{a}$ in $\mathrm{CDCl}_{3}$ at room temperature

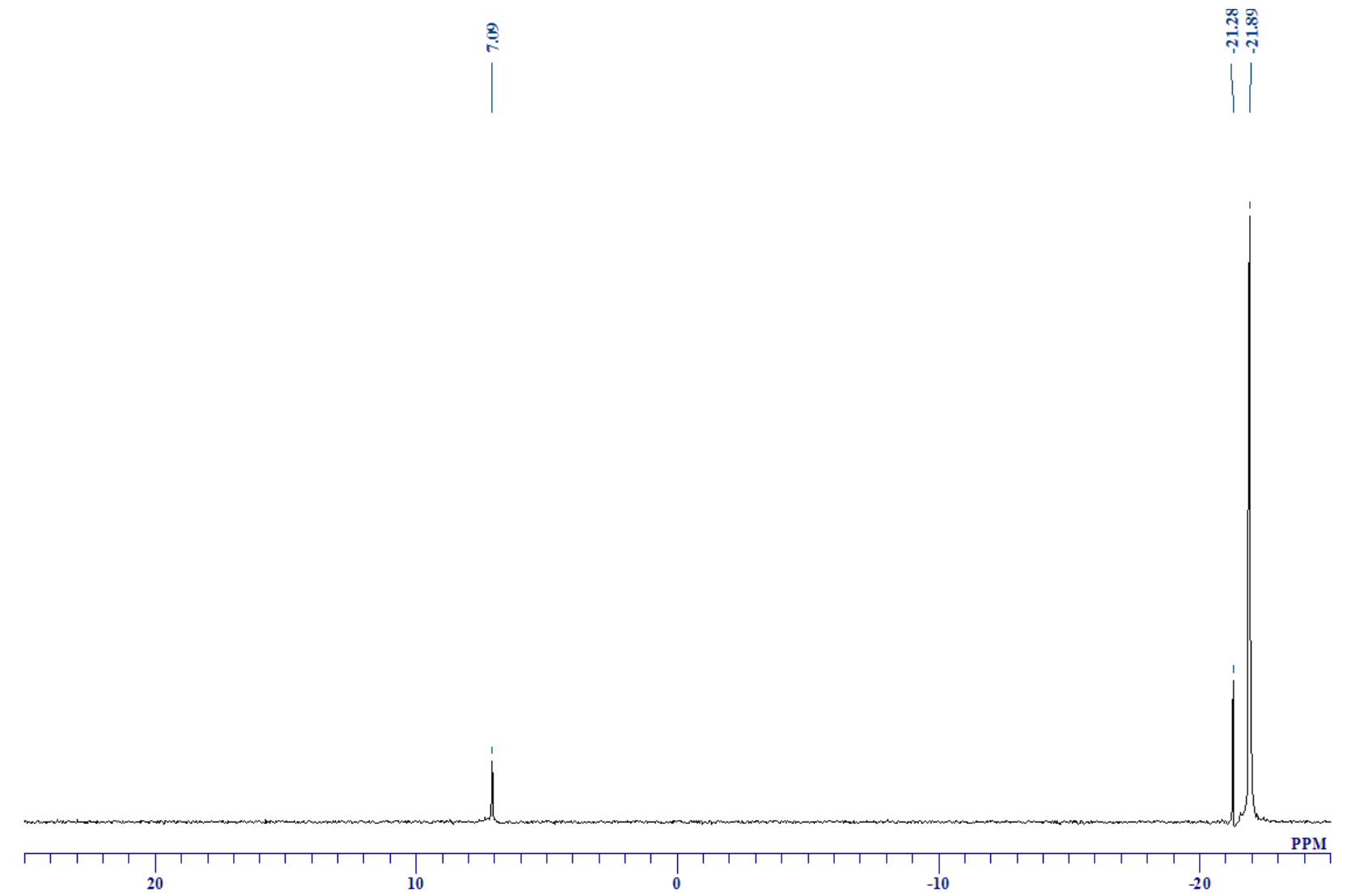

Figure S30-1. ${ }^{1} \mathrm{H}$ NMR spectrum of a solution of 26 a in $\mathrm{CDCl}_{3}$ at room temperature
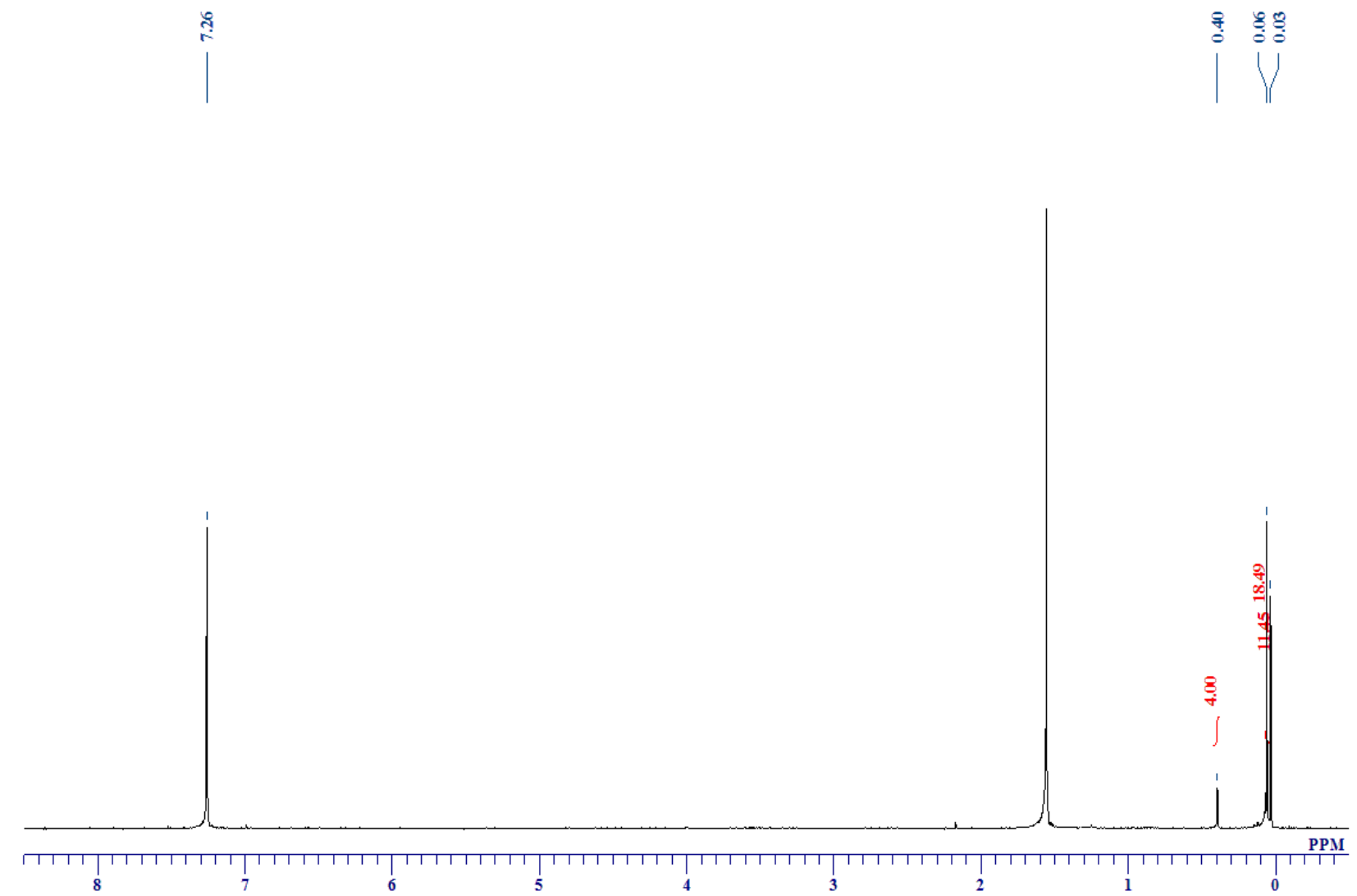
Figure $\mathrm{S} 30-2 .{ }^{13} \mathrm{C} \mathrm{NMR}$ spectrum of a solution of $26 \mathrm{a}$ in $\mathrm{CDCl}_{3}$ at room temperature

$$
\text { 낭 }
$$
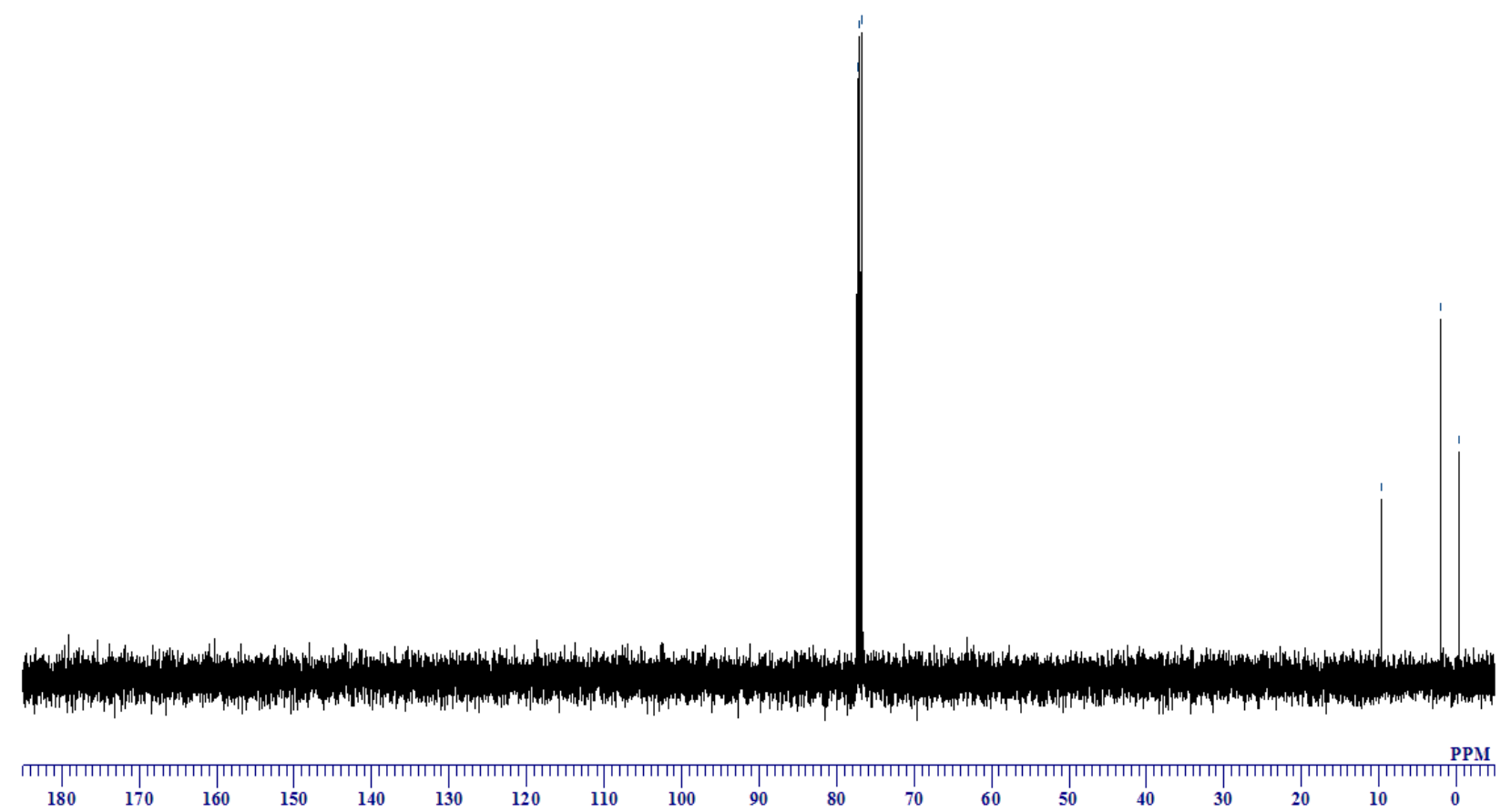

Figure S30-3. ${ }^{29} \mathrm{Si}$ NMR spectrum of a solution of $26 \mathrm{a}$ in $\mathrm{CDCl}_{3}$ at room temperature
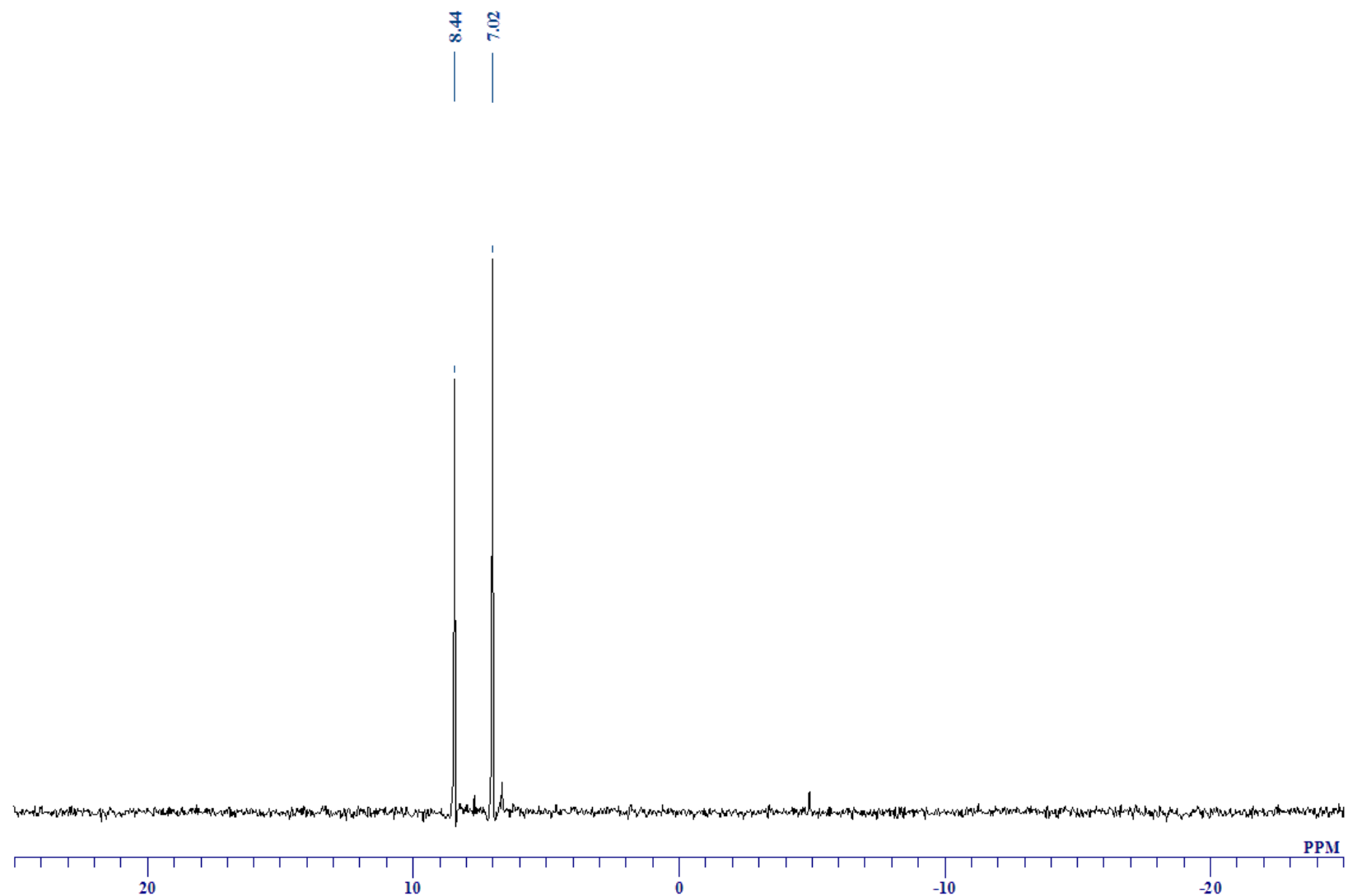
Figure S31-1. ${ }^{1} \mathrm{H}$ NMR spectrum of a solution of $27 \mathrm{a}$ in $\mathrm{CDCl}_{3}$ at room temperature

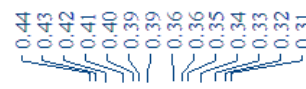

8.88
11000
000
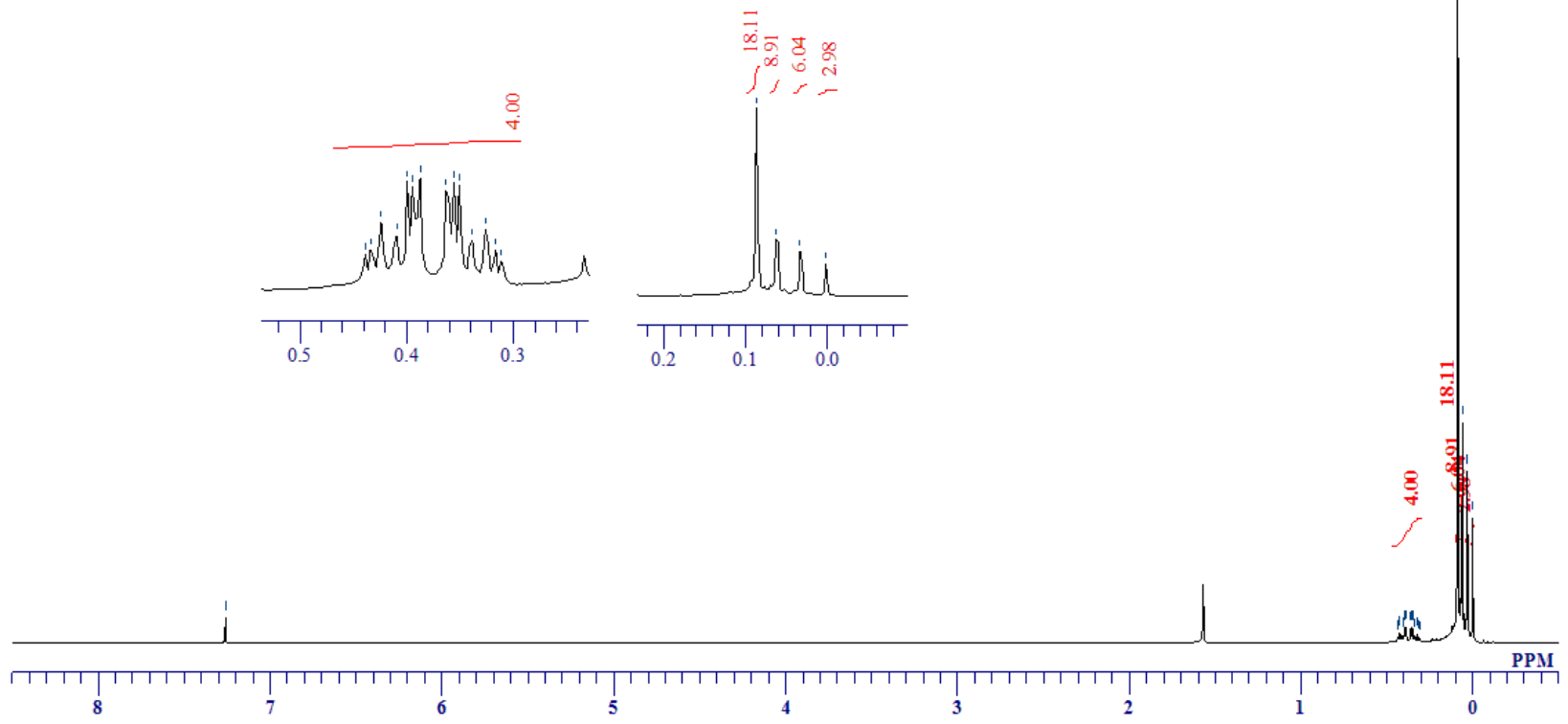

Figure S31-2. ${ }^{13} \mathrm{C}$ NMR spectrum of a solution of $27 \mathrm{a}$ in $\mathrm{CDCl}_{3}$ at room temperature<smiles>C1#CC2C=CC(C1)C2</smiles>
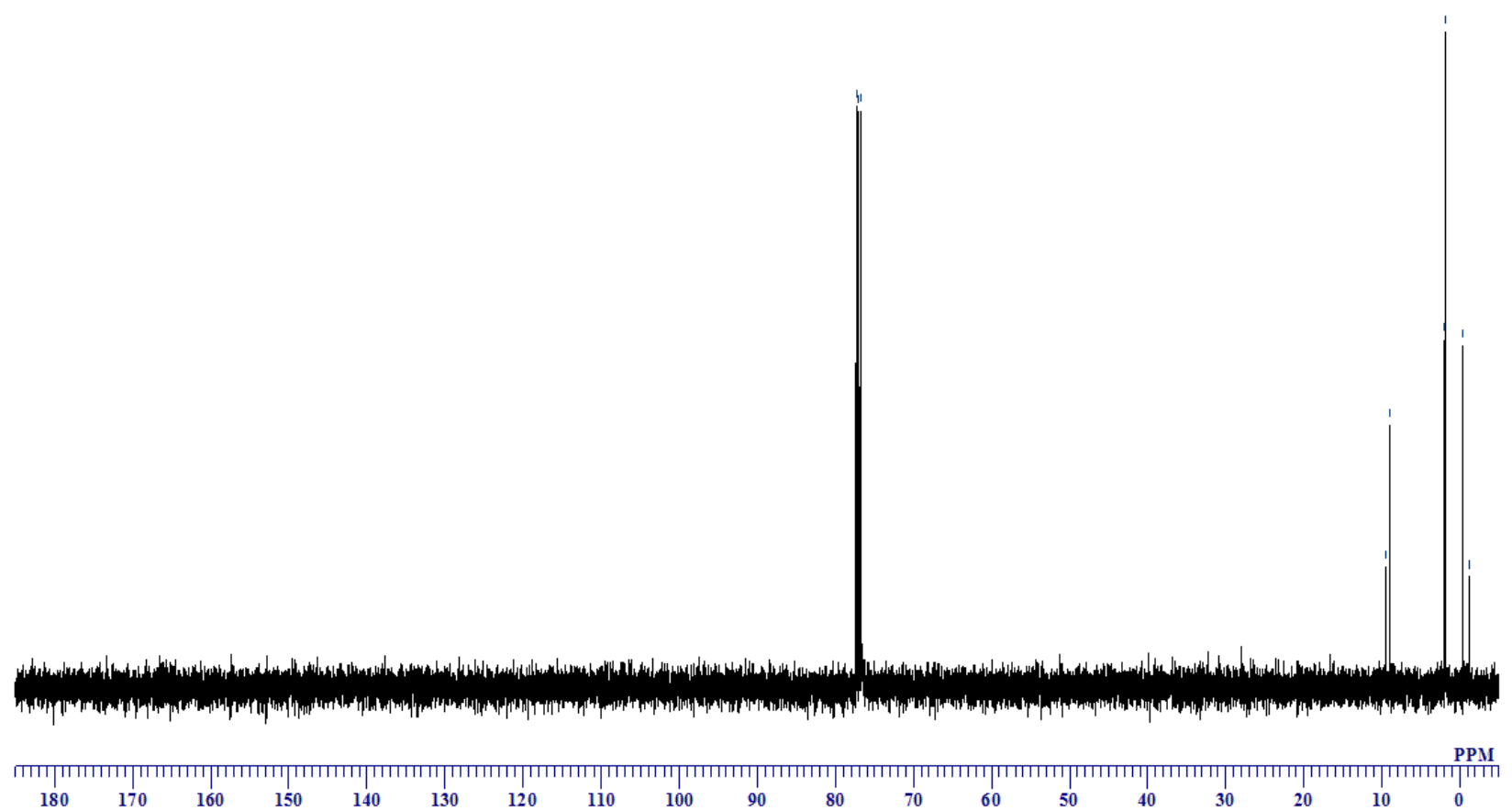
Figure S31-3. ${ }^{29} \mathrm{Si} \mathrm{NMR}$ spectrum of a solution of $27 \mathrm{a}$ in $\mathrm{CDCl}_{3}$ at room temperature
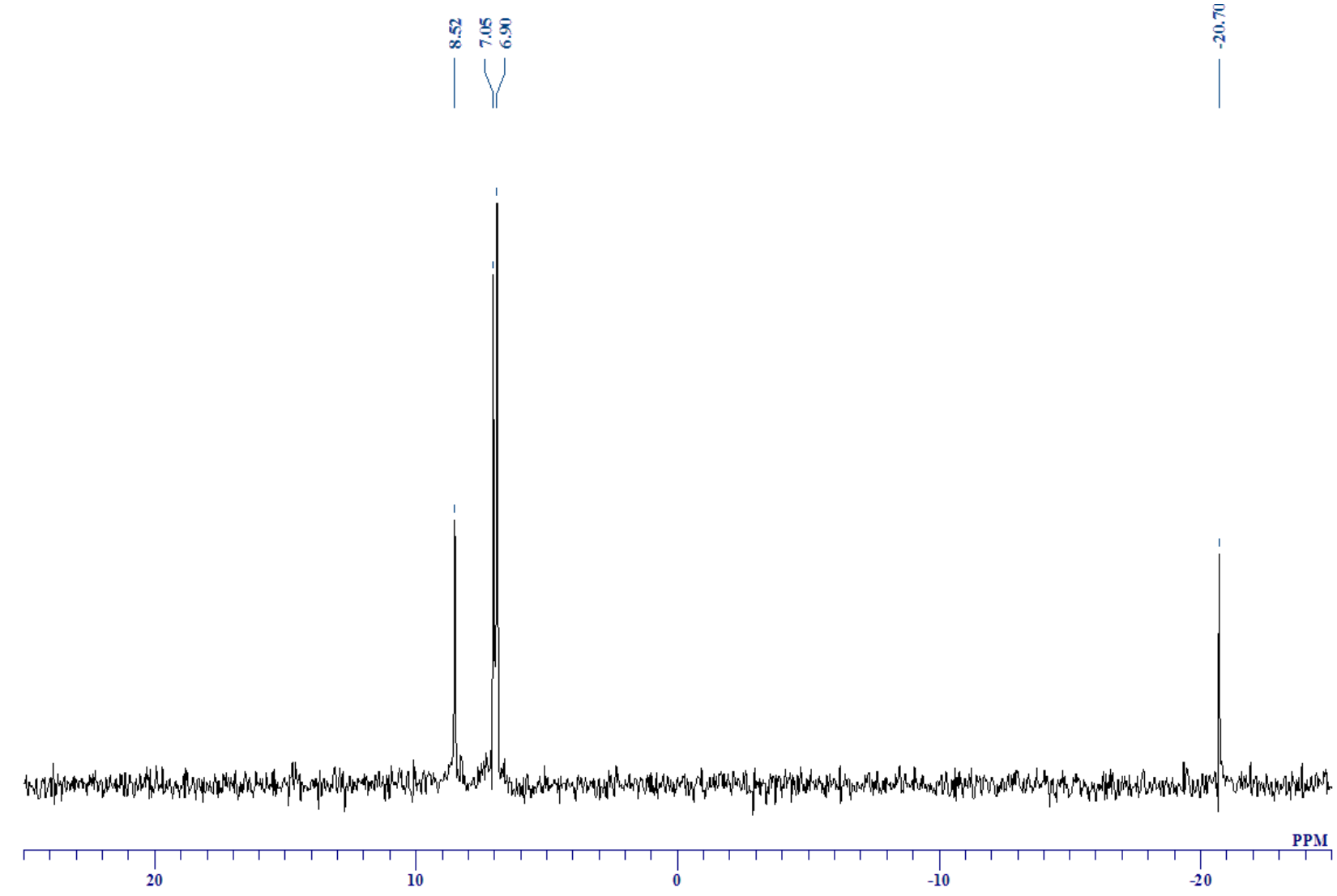

Figure $\mathrm{S} 32-1 .{ }^{1} \mathrm{H}$ NMR spectrum of a solution of $28 \mathrm{a}$ in $\mathrm{CDCl}_{3}$ at room temperature
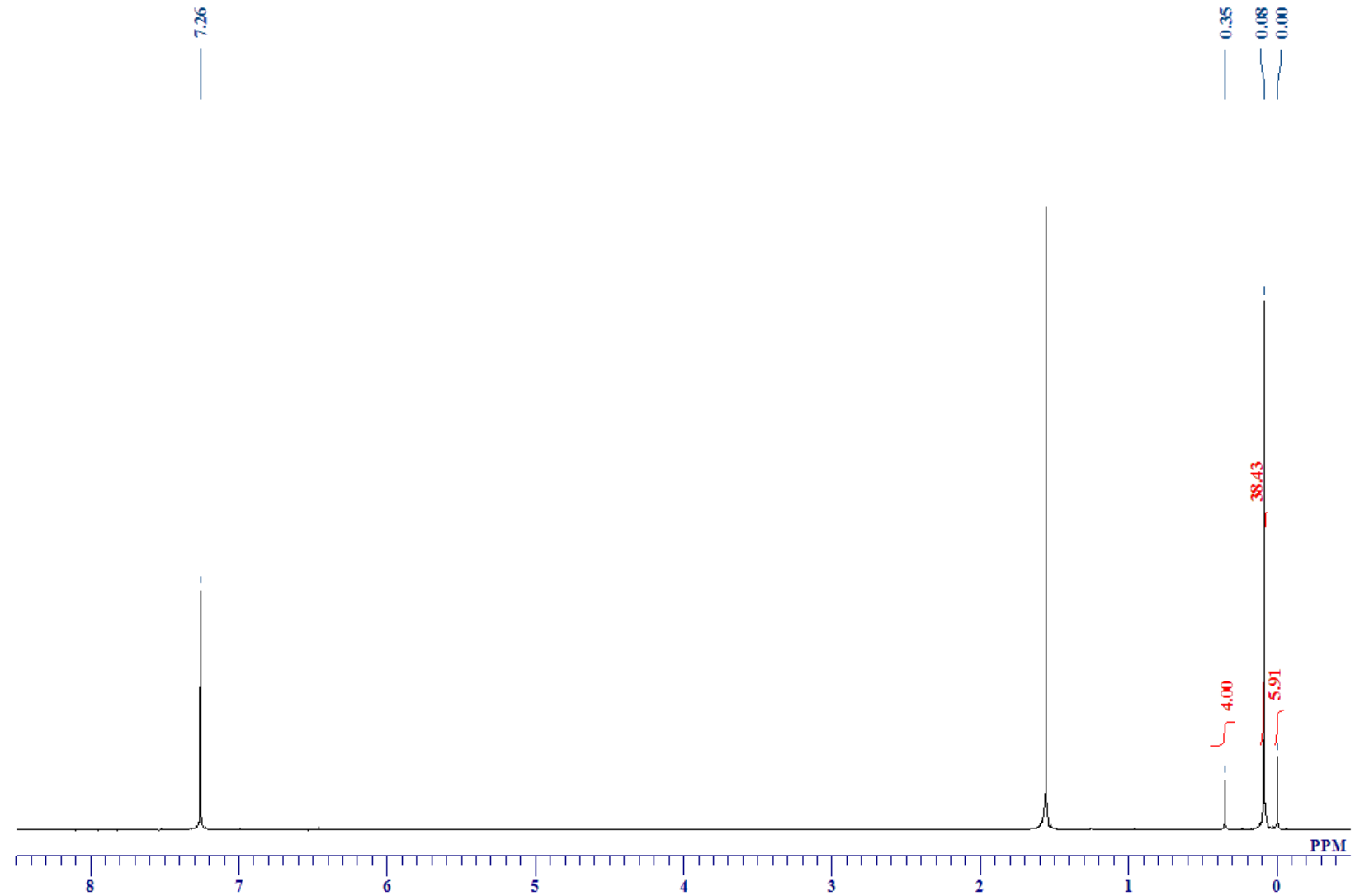
Figure S32-2. ${ }^{13} \mathrm{C}$ NMR spectrum of a solution of $28 \mathrm{a}$ in $\mathrm{CDCl}_{3}$ at room temperature
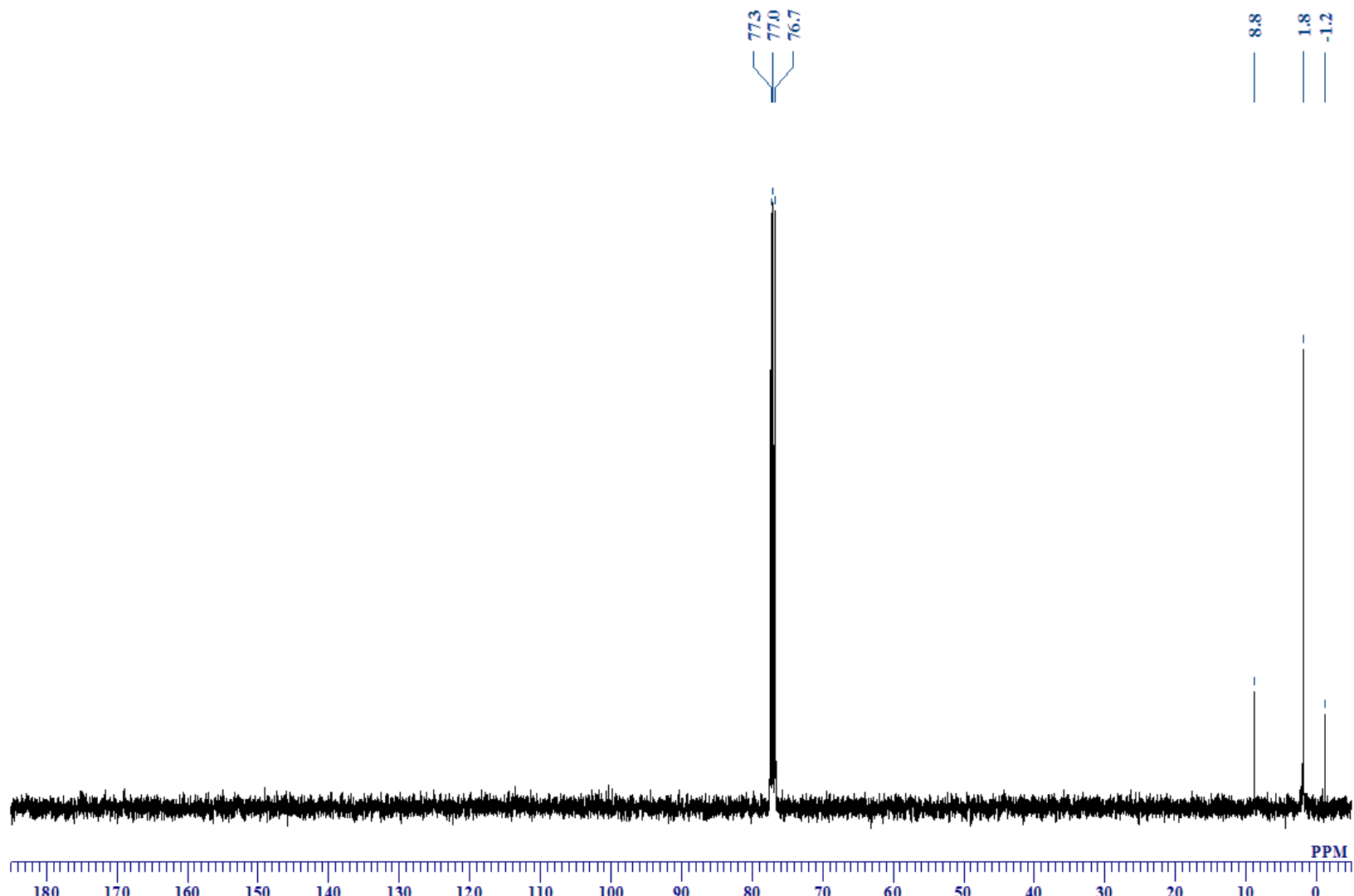

Figure S32-3. ${ }^{29} \mathrm{Si} \mathrm{NMR}$ spectrum of a solution of $28 \mathrm{a}$ in $\mathrm{CDCl}_{3}$ at room temperature

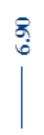

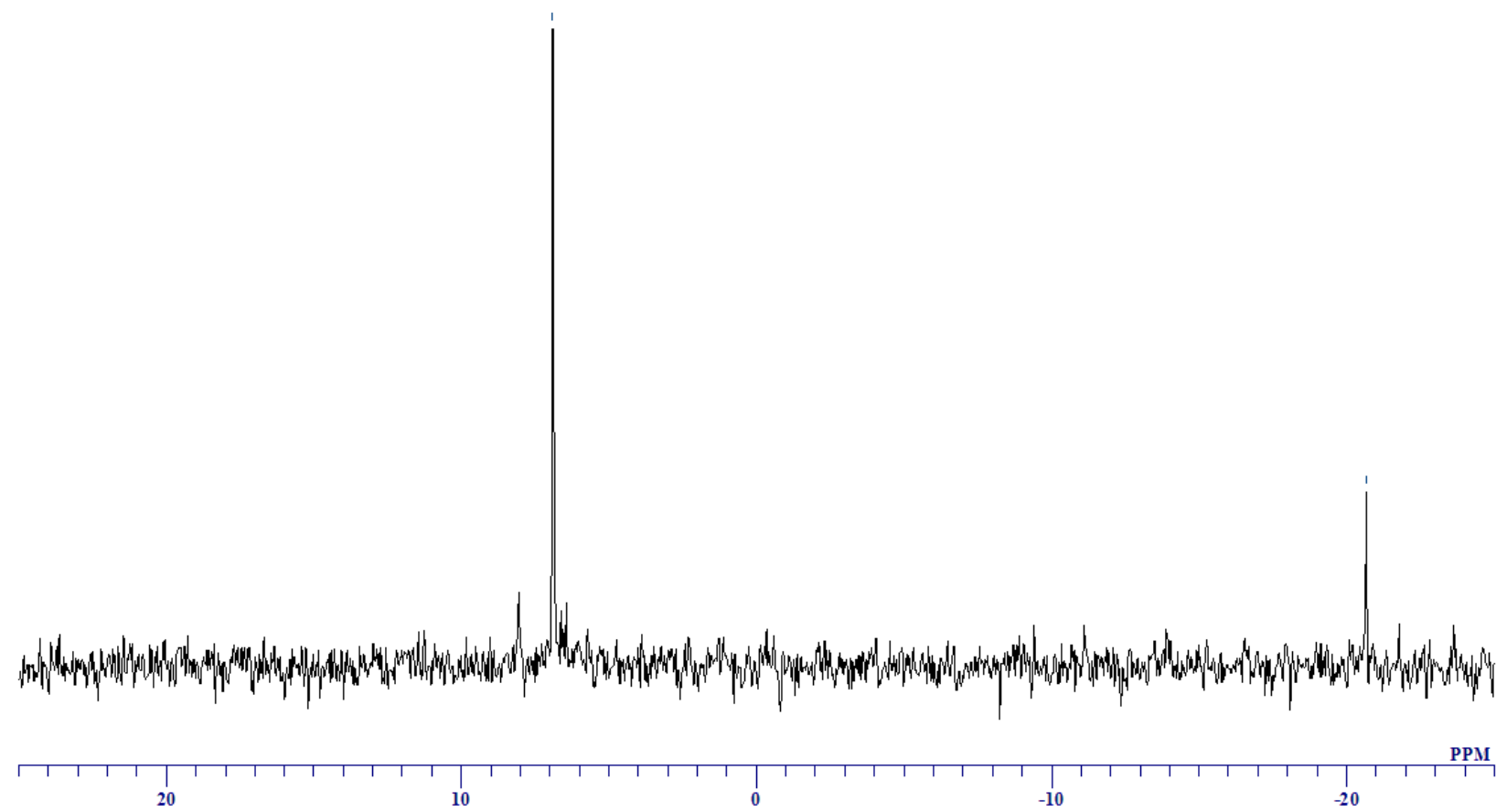


Figure S33-1. ${ }^{1} \mathrm{H}$ NMR spectrum (measured in $\mathrm{CDCl}_{3}$, r.t) of the reaction mixture obtained from the reaction of 3-(2-methoxyethoxy)-prop-1-ene with PMDS.
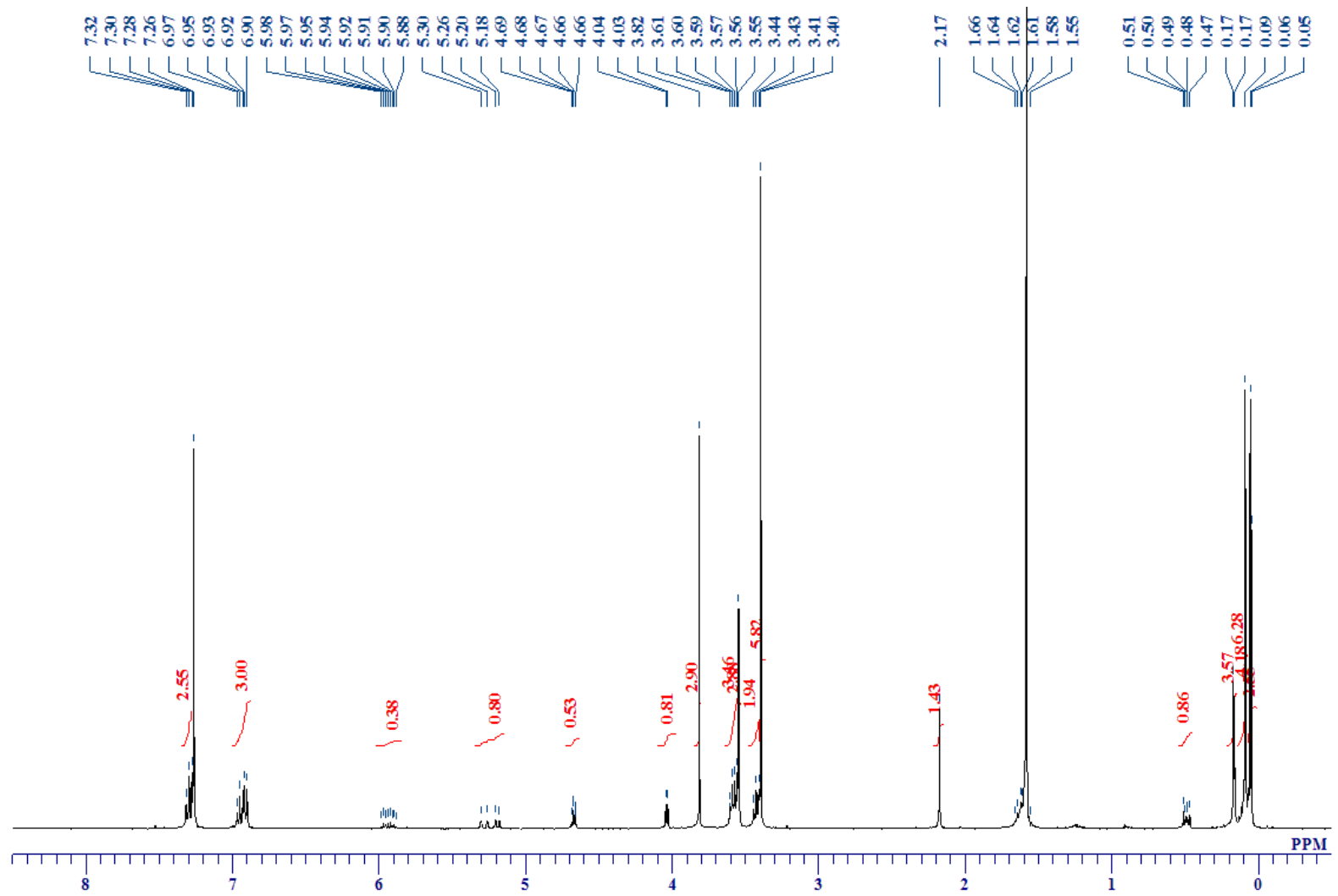

Figure S33-2. ${ }^{1} \mathrm{H}$ NMR spectrum (measured in $\mathrm{CDCl}_{3}$, r.t) of the isolated hydrosilylated product obtained from the reaction of 3-(2-methoxyethoxy)-prop-1-ene with PMDS.

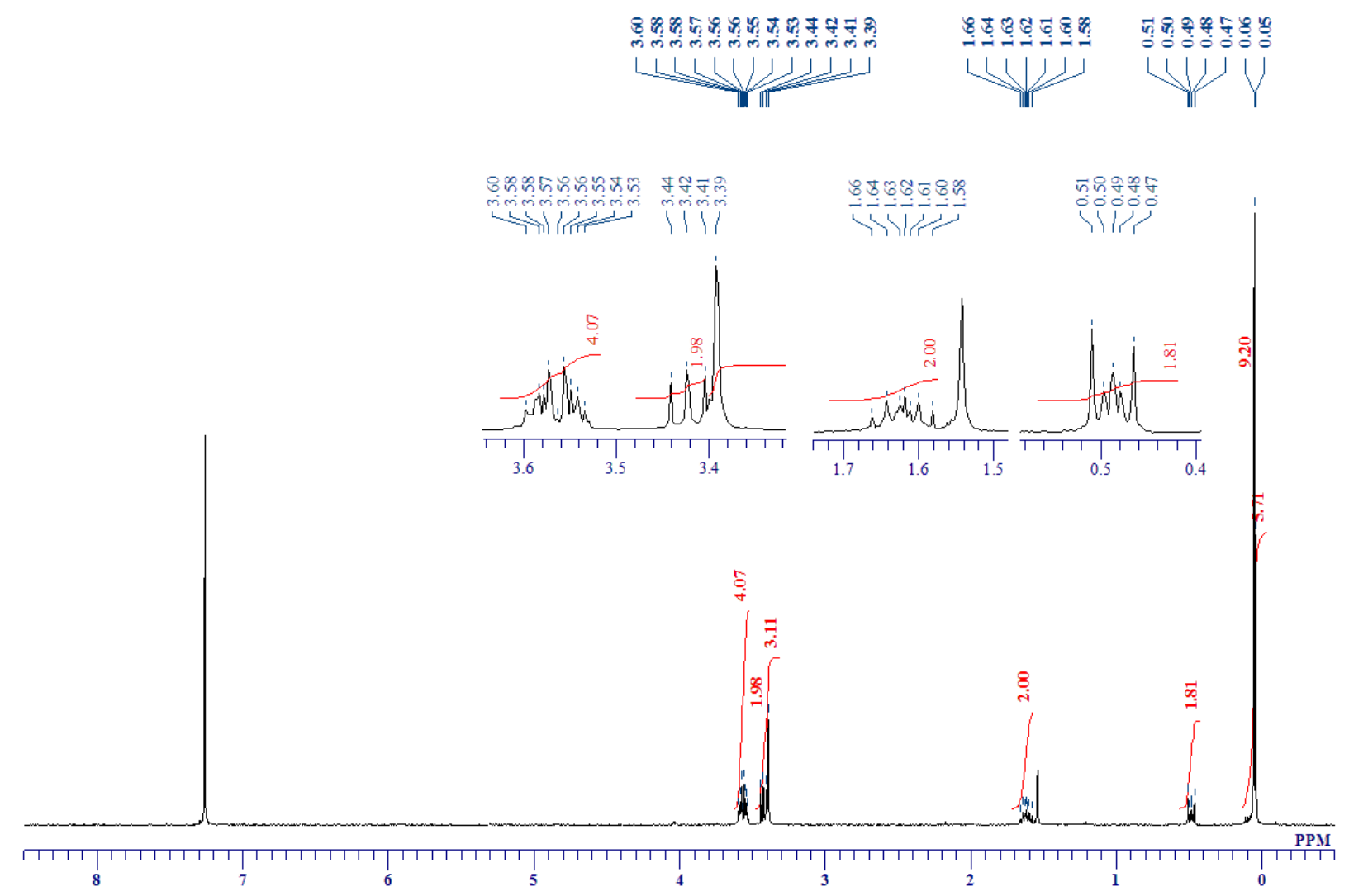


Figure S33-3. ${ }^{13} \mathrm{C}$ NMR spectrum (measured in $\mathrm{CDCl}_{3}$, r.t) of the isolated hydrosilylated product obtained from the reaction of 3-(2-methoxyethoxy)-prop-1-ene with PMDS.

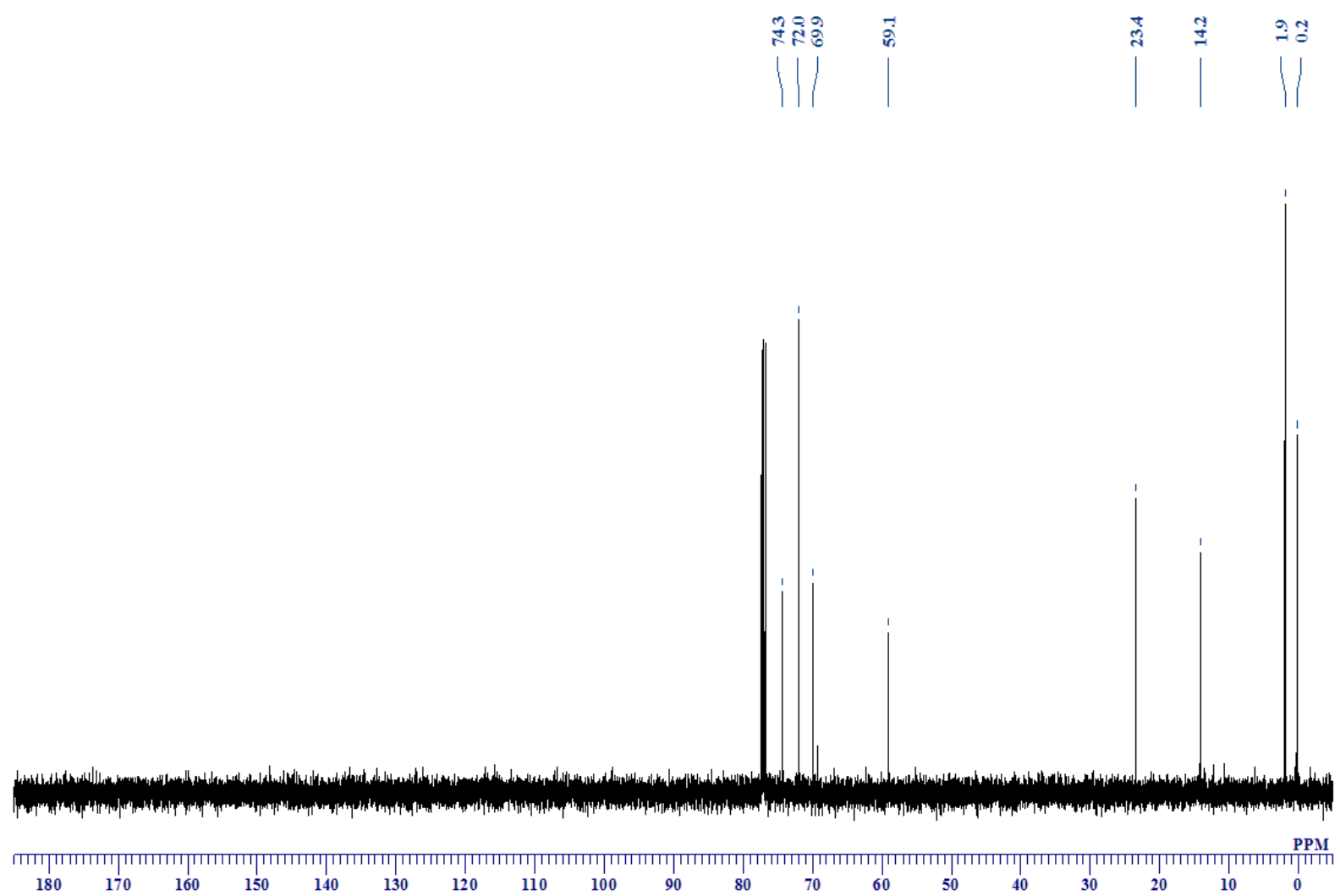

Figure S33-4. ${ }^{29} \mathrm{Si} \mathrm{NMR}$ spectrum (measured in $\mathrm{CDCl}_{3}$, r.t) of the isolated hydrosilylated product obtained from the reaction of 3-(2-methoxyethoxy)-prop-1-ene with PMDS.

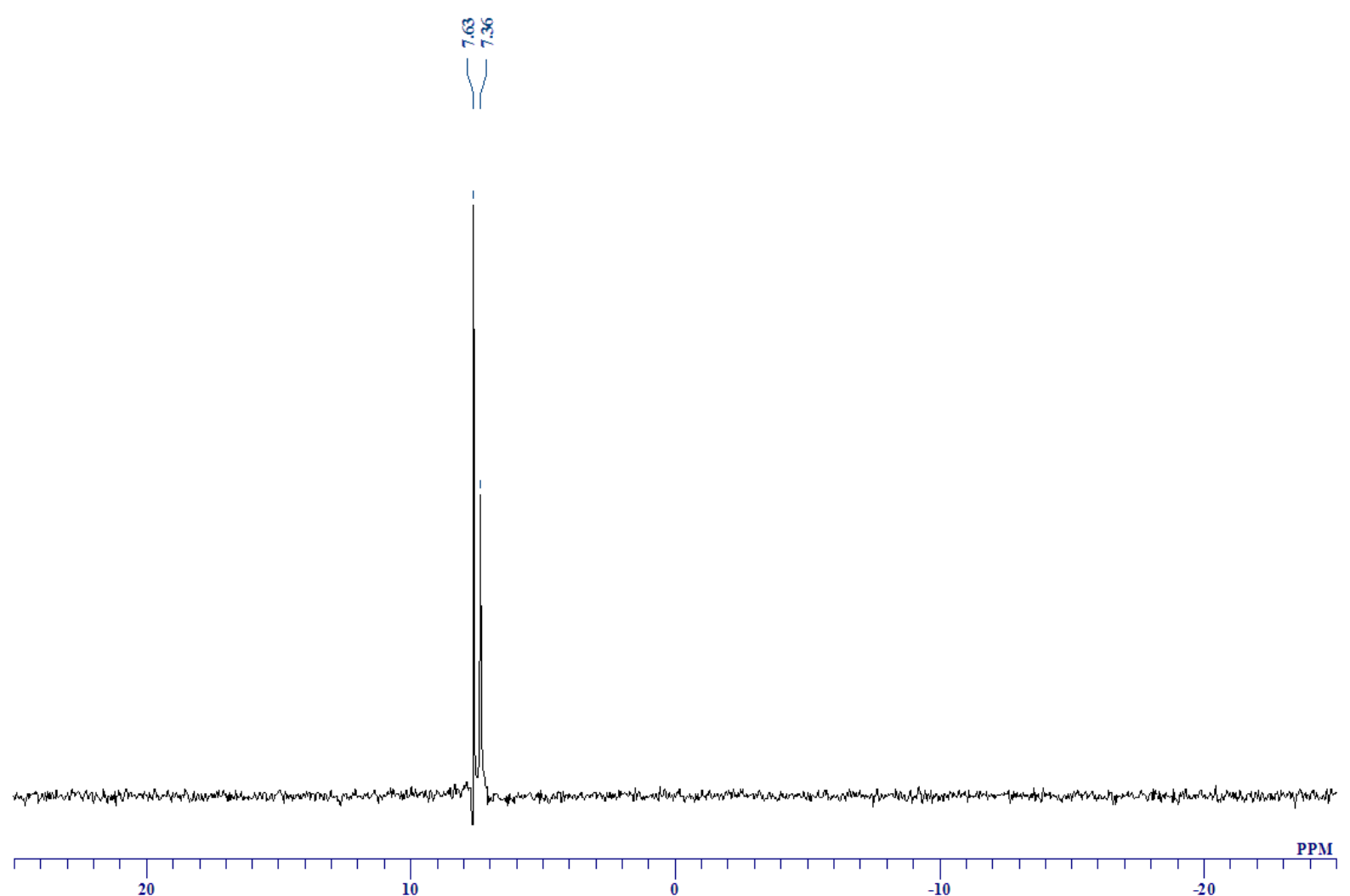


Figure S34-1. ${ }^{1} \mathrm{H}$ NMR spectrum (measured in $\mathrm{CDCl}_{3}$, r.t) of the reaction mixture obtained from the reaction of $\mathrm{CH}_{2}=\mathrm{CHCH}_{2}\left(\mathrm{OCH}_{2} \mathrm{CH}_{2}\right)_{n} \mathrm{OMe}(\mathrm{n}=8)$ with PMDS.

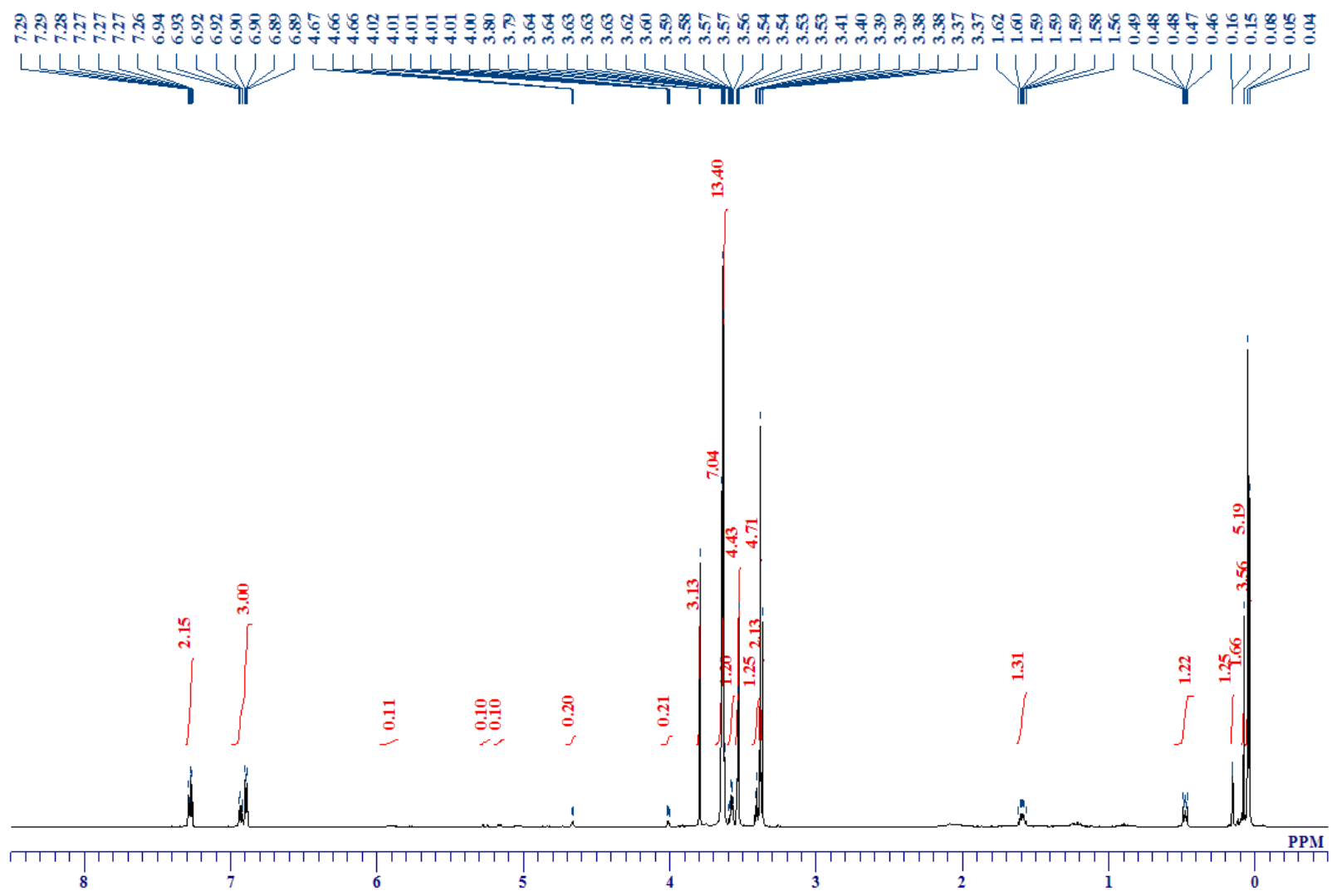

